BNL-75554-2006

\title{
DUSTMS-D
}

\section{DISPOSAL UNIT SOURCE TERM - MULTIPLE SPECIES - DISTRIBUTED FAILURE DATA INPUT GUIDE}

\author{
T. M. Sullivan
}

January 2006

\author{
Environmental Sciences Department \\ Environmental Research \& Technology Division \\ Brookhaven National Laboratory \\ P.O. Box 5000 \\ Upton, NY 11973-5000 \\ www.bnl.gov
}




\section{DISCLAIMER}

This report was prepared as an account of work sponsored by an agency of the United States Government. Neither the United States Government nor any agency thereof, nor any of their employees, nor any of their contractors, subcontractors, or their employees, makes any warranty, express or implied, or assumes any legal liability or responsibility for the accuracy, completeness, or any third party's use or the results of such use of any information, apparatus, product, or process disclosed, or represents that its use would not infringe privately owned rights. Reference herein to any specific commercial product, process, or service by trade name, trademark, manufacturer, or otherwise, does not necessarily constitute or imply its endorsement, recommendation, or favoring by the United States Government or any agency thereof or its contractors or subcontractors. The views and opinions of authors expressed herein do not necessarily state or reflect those of the United States Government or any agency thereof. 


\begin{abstract}
Performance assessment of a low-level waste (LLW) disposal facility begins with an estimation of the rate at which radionuclides migrate out of the facility (i.e., the source term). The focus of this work is to develop a methodology for calculating the source term. In general, the source term is influenced by the radionuclide inventory, the wasteforms and containers used to dispose of the inventory, and the physical processes that lead to release from the facility (fluid flow, container degradation, wasteform leaching, and radionuclide transport). Many of these physical processes are influenced by the design of the disposal facility (e.g., how the engineered barriers control infiltration of water). The complexity of the problem and the absence of appropriate data prevent development of an entirely mechanistic representation of radionuclide release from a disposal facility. Typically, a number of assumptions, based on knowledge of the disposal system, are used to simplify the problem. This has been done and the resulting models have been incorporated into the computer code DUST-MS (Disposal Unit Source Term - Multiple Species). The DUST-MS computer code is designed to model water flow, container degradation, release of contaminants from the wasteform to the contacting solution and transport through the subsurface media. Water flow through the facility over time is modeled using tabular input. Container degradation models include three types of failure rates: a) instantaneous (all containers in a control volume fail at once), b) uniformly distributed failures (containers fail at a linear rate between a specified starting and ending time), and c) gaussian failure rates (containers fail at a rate determined by a mean failure time, standard deviation and gaussian distribution). Wasteform release models include four release mechanisms: a) rinse with partitioning (inventory is released instantly upon container failure subject to equilibrium partitioning (sorption) with the waste form), b) diffusion release.(release from either a cylindrical, spherical, or rectangular wasteform), c) dissolution release (uniform release over time due to dissolution of the wasteform surface), and d) solubility limited release. The predicated wasteform releases are corrected for radioactive decay and ingrowth. A unique set of container failure and wasteform release parameters can be specified for each control volume with a container. Contaminant transport is modeled through a finite-difference solution of the advective transport equation with sources (wasteform release and ingrowth) and radioactive decay. Although DUST-MS simulates one-dimensional transport, it can be used to simulate migration down to an aquifer and then transport in the aquifer by running the code twice. A special subroutine allows the flux into the aquifer from the first simulation to be input as the flux at the upstream boundary in the aquifer. This document presents the models used to calculate release from a disposal facility, verification of the model, and instructions on the use of the DUST-MS code. In addition to DUST-MS, a preprocessor, DUSTINMS, which helps the code user create input decks for DUST-MS and a post-processor, GRAFMS, which takes selected output files and plots them on the computer terminal have been written. Use of these codes is also described. In using DUST-MS, as with all computer models, the validity of the predictions relies heavily on the validity of the input parameters. Often, the largest uncertainties arise from uncertainty in the input parameters. Therefore, it is crucial to document and support the use of these parameters. The DUST-MS code, because of its flexibility and ability to compute release rates quickly, is extremely useful for screening to determine the radionuclide released at the highest rate, parameter sensitivity analysis and, with proper choice of the input parameters, provide upper bounds to release rates.
\end{abstract}




\section{TABLE OF CONTENTS}

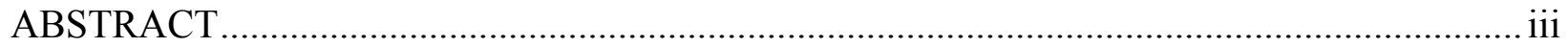

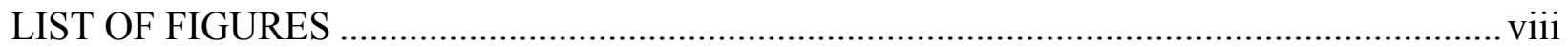

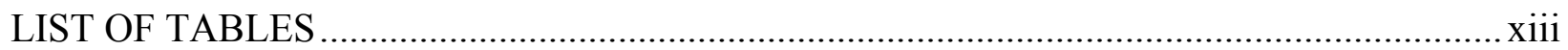

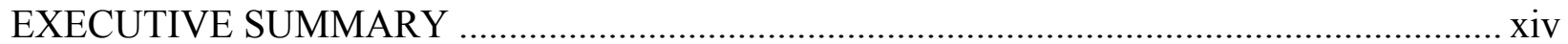

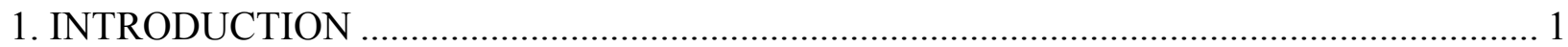

2. MODEL SELECTION AND GOVERNING EQUATIONS ……....................................... 1

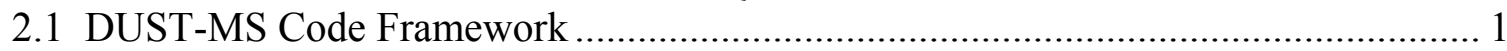

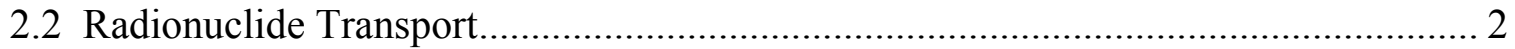

2.2.1 Transport Equation with Chain Decay ............................................................... 3

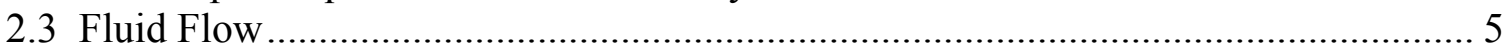

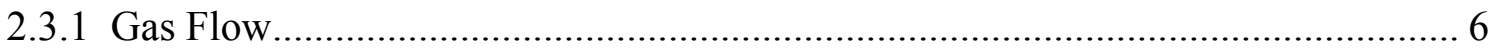

2.4 Container Degradation .................................................................................. 7

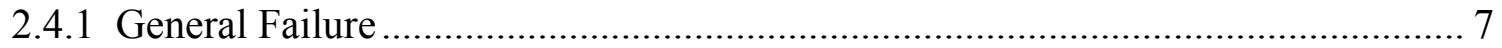

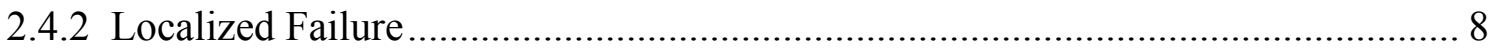

2.4.3 Distributed Container Failure Models (DUSTMS-D code) .................................... 9

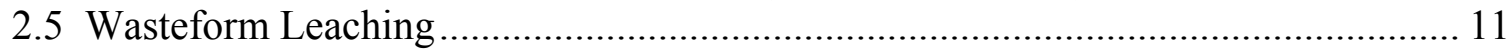

2.5 Waste Form Release Models ……………….................................................... 12

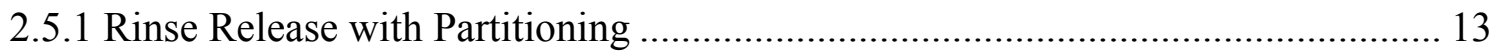

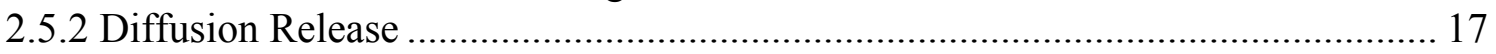

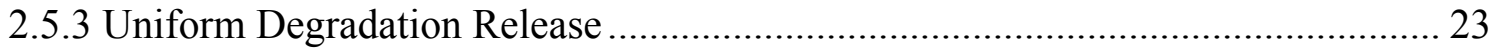

2.5.4 Total Waste-Form Release Rate .................................................................... 23

2.5.5 Finite Difference Waste-Form Release Model.................................................. 24

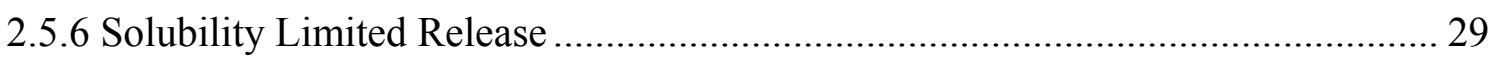

2.5.7 Treatment of Chain Decay in the Waste-Form ..................................................... 30

2.5.9 Selection of Release Models ............................................................................. 36

2.5.10 Wasteform Release with Distributed Container Failure..................................... 33

2.6 Transport Equation Initial and Boundary Conditions.............................................. 37

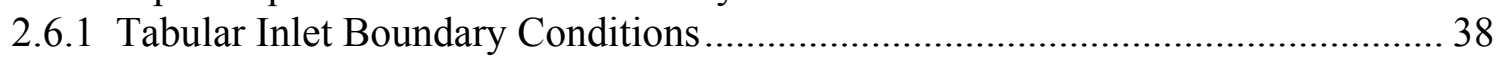

3. PROCEDURE FOR CALCULATING RELEASES USING DUST-MS ................................ 41

4. APPLICABILITY AND LIMITATIONS OF THE DUST CODE ………………............... 45

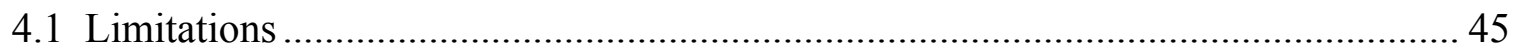

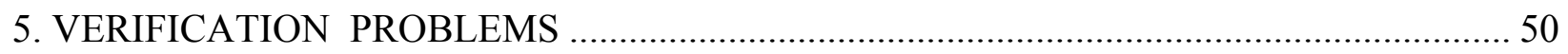

5.1 One-Dimensional Transport of a Single Three-Member Decay Chain ..................... 50

5.2 One-dimensional Transport with Two Decay Chains................................................. 54

5.3 Ingrowth of Progeny Prior to Release from the Waste Form …………………….... 57

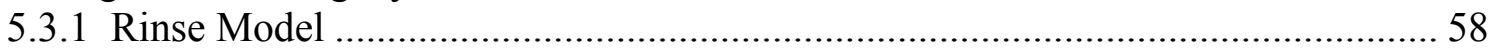

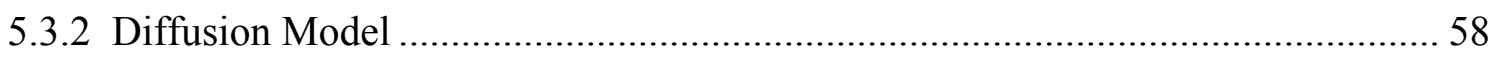

5.3.3 Uniform Degradation Model ........................................................................... 66

6. DUSTINMS: A PREPROCESSOR THAT CREATES AN INPUT DECK FOR DUST-MS 70

6.1 General Problem Definitions.......................................................................... 76

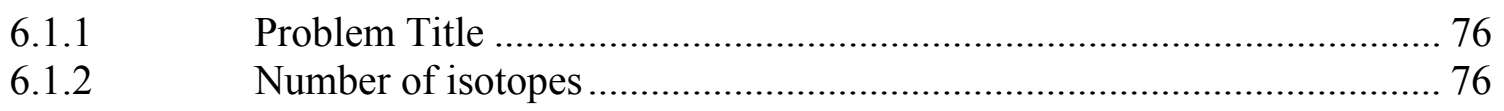




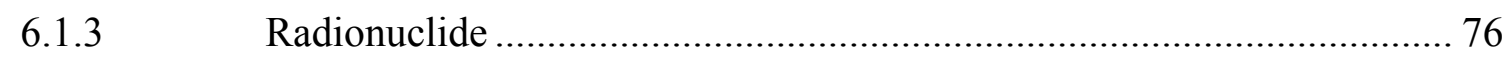

6.1.4 Units Flag for Mass Input ................................................................... 77

6.1.5 Number of Computational Cells ………….............................................. 77

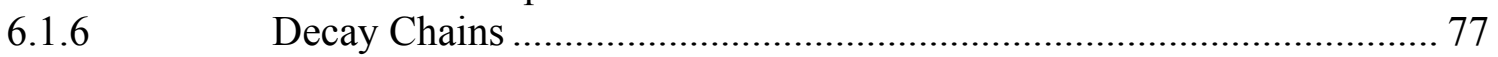

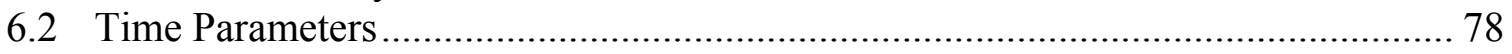

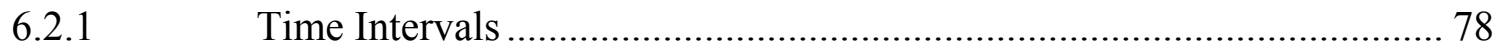

6.2.2 Number of Time Step Changes ............................................................ 78

6.2.3 Output Times or Time Step Changes................................................... 79

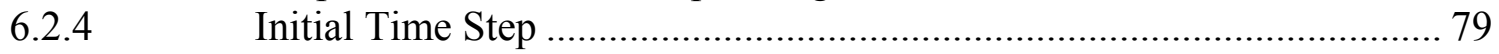

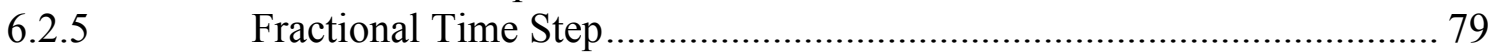

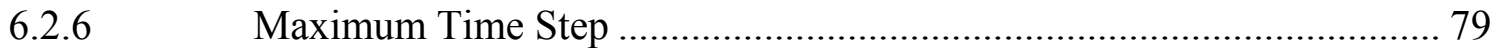

6.2.7 Maximum Simulation Time _................................................................... 80

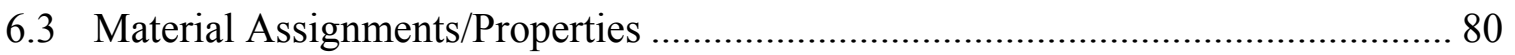

6.3.1 Number of Different Materials ………................................................ 80

6.3.2 Material Type Assignments ................................................................. 80

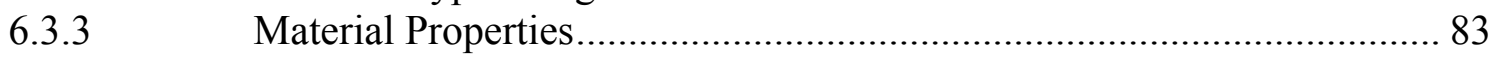

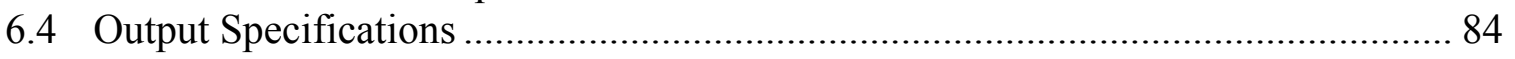

6.4.1 Printer Control Parameters................................................................... 85

6.4.2 Number of Concentration Traces.......................................................... 86

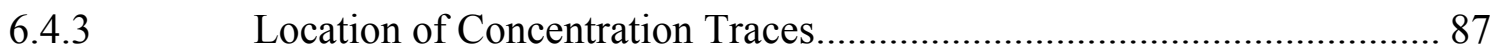

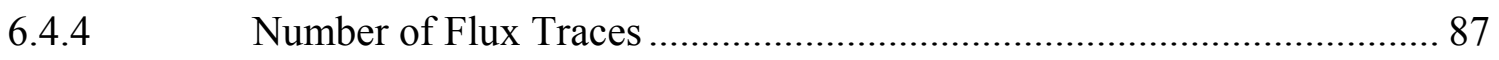

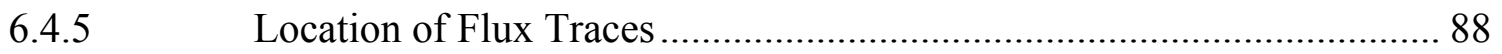

6.4.6 Number of Time Steps between Traces .............................................. 88

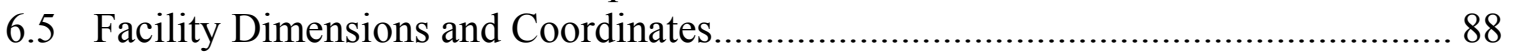

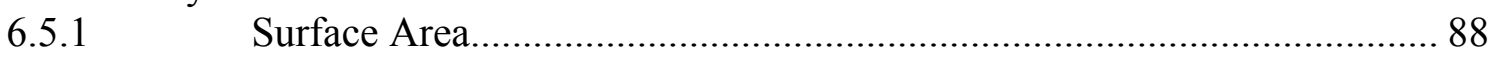

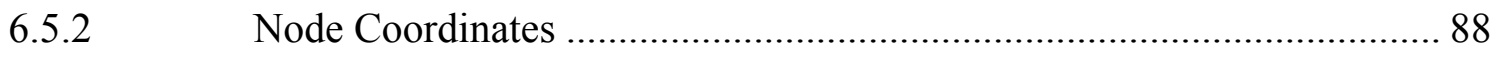

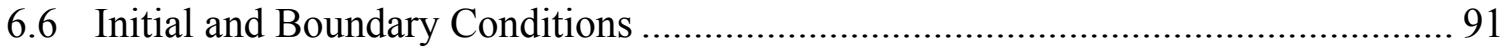

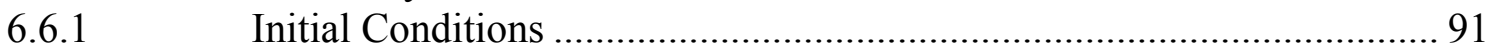

6.6.2 Top Boundary Condition Flag .............................................................. 93

6.6.3 Bottom Boundary Condition Flag.......................................................... 93

6.6.4 Number of Boundary Condition Data Points.......................................... 93

6.6.5 Boundary Condition Table for the Top Boundary .................................... 94

6.6.6 Boundary Condition Table for the Bottom Boundary ……………......... 95

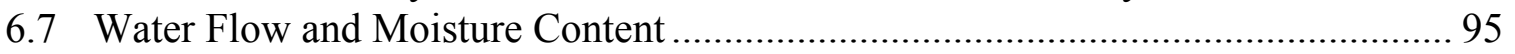

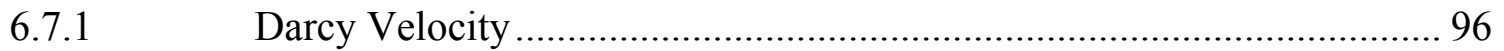

6.7.2 Moisture Content Values ...................................................................... 97

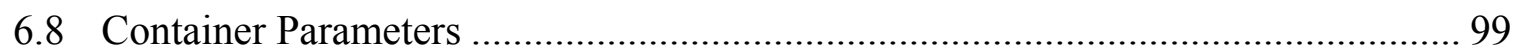

6.8.1 Number of Containers.................................................................. 100

6.8.2 Time of General Failure.................................................................. 100

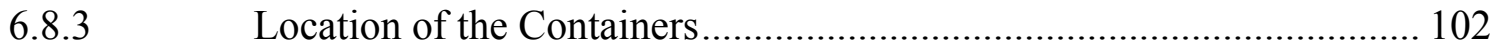

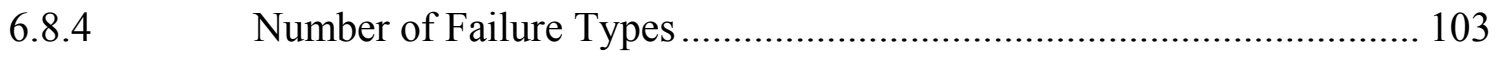

6.8.5 Corrosion Model Flag ………………............................................. 103

6.8.6 Localized Failure Parameters................................................................ 103

6.8.7 Assignment of Localized Failure Parameters to Each Container .......... 106 


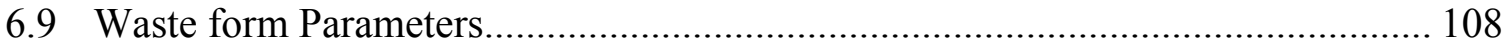

6.9.1 Number of Waste Types ………………............................................ 109

6.9.2 Finite Difference Model Release Rate Parameters ............................... 109

6.9.3 Mixing Cell Model Release Rate Parameters ........................................... 114

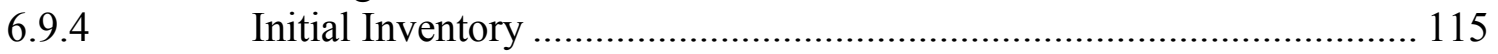

6.9.5 Waste Type Assignment to Each Container ………………………........ 116

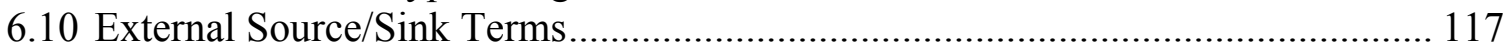

6.10.1 Number of Source Nodes............................................................... 118

6.10.2 Number of Source Profiles.............................................................. 118

6.10.3 Number of Source Data Points ........................................................ 118

6.10.4 Times and Values of the Sources...................................................... 118

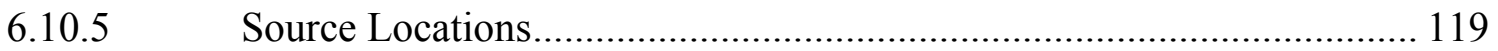

6.10.6 Source Type Assignments.................................................................... 120

6.11 Create an Input Deck for Use by the DUST-MS Code ......................................... 121

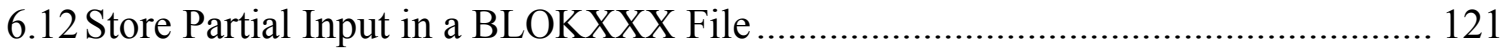

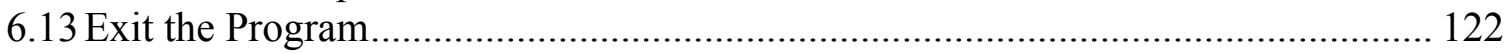

7. STRUCTURE OF A DUST-MS and DUSTMS-D INPUT DECK ………………............... 123

7.1 DATA SET 1: Title and General Problem Definition (Menu 1) ………………..... 129

7.2 DATA SET 2: Time Parameters (Menu 2) …………………………………....... 131

7.3 DATA SET 3: Material Assignments/Properties (Menu 3) ................................. 132

7.4 DATA SET 4: Output Specifications (Menu 4) ………………………………..... 134

7.5 DATA SET 5: Facility Co-ordinate Data (Menu 5)............................................... 136

7.6 DATA SET 6: Initial and Boundary Conditions (Menu 6) ……………………..... 137

7.7 DATA SET 7: Water Flow Parameters (Menu 7) ……………………………....... 139

7.8 DATA SET 8: Container Parameters (Menu 8)................................................... 140

7.9 DATA SET 9: Wasteform Leaching Parameters ………………………………..... 145

7.10 DATA SET 10: External Sources .............................................................. 148

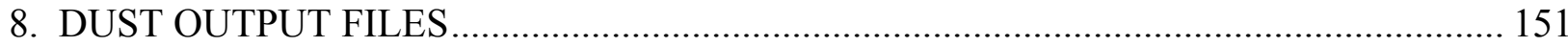

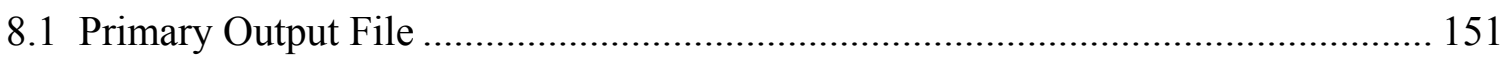

8.2 Concentration Trace File (TRCCND?.DAT) ………........................................... 158

8.3 Flux Trace File (TRCFXD?.DAT) ………………........................................ 160

8.4 Concentration File (CONCNT.DAT) ……………............................................ 163

8.5 Wasteform Release Data (LEACHRL.DAT) ………………………………........ 165

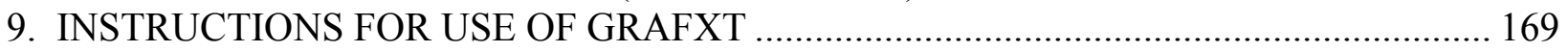

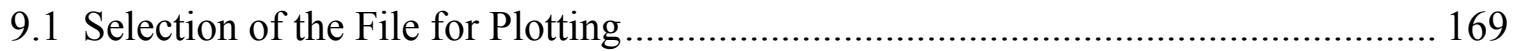

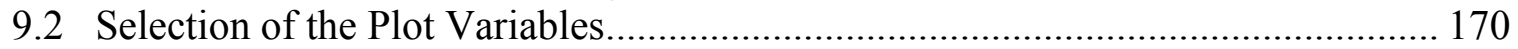

9.2.1 Number of plots per graph - Independent variable is time................................. 171

9.2.2 Number of plots per graph - Independent variable is distance............................ 172

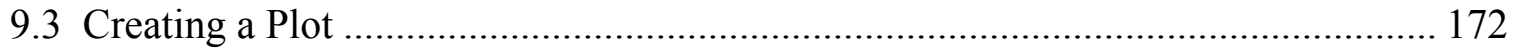

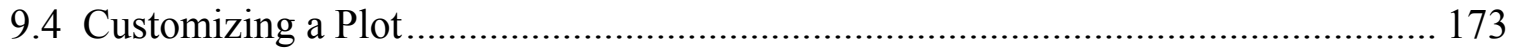

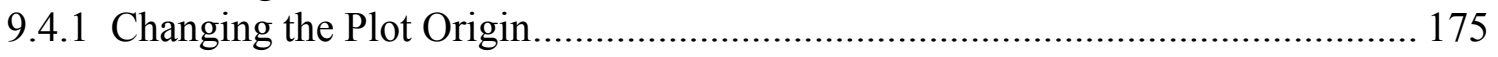

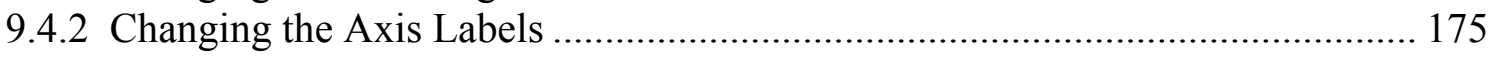

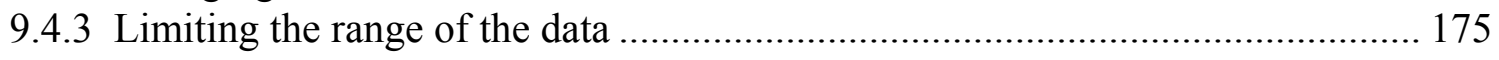

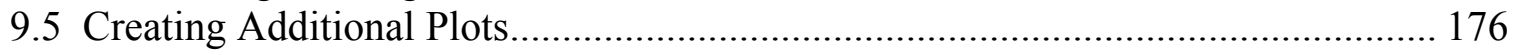


10. CONCLUSIONS.

APPENDIX A: MATHEMATICAL DESCRIPTION OF THE MODELS SELECTED FOR

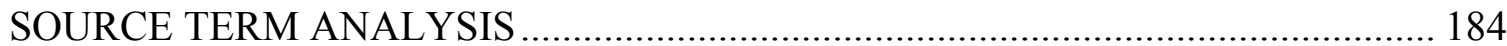

APPENDIX C: FLOW CHART FOR THE DUST-MS CODE ……………………................ 1 APPENDIX D: DATA REQUIREMENTS FOR THE PITTING MODEL IN SUBROUTINE

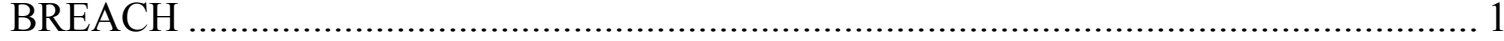




\section{LIST OF FIGURES}

$\underline{\text { PAGE }}$

Figure 1.1 Schematic diagram of a LLW Disposal Unit $1-2$

Figure 2.1 Schematic representation of the mixing cell cascade approach.

(a) a single mixing cell, (b) multiple mixing cells

Figure 3.1 Flowchart of the procedure used to take waste stream/wasteform/ container inventory data and define the necessary input parameters to estimate the release rate from a disposal facility

Figure 3.2 Schematic outline of the procedure used to analyze release from a LLW disposal facility using the DUST code package.

Figure 5.1 Test case 1, comparison of the DUST finite difference model predictions with the analytical solution for a pulse source at $\mathrm{x}=0$ for 5 days. Retardation and decay are modeled.

Figure 5.2 Test case 2, comparison of the DUST finite difference model predictions with the analytical solution for a pulse source at $\mathrm{x}=0$ for 5 days. The decay term is zero in this simulation.

Figure 5.3 Test case 3, comparison of the DUST finite difference model predictions with the analytical solution for a uniform initial concentration, zero total flux at the boundary $\mathrm{x}=0$, and an external uniform source.

Figure 5.4 Test case 4, comparison of the DUST finite difference and mixing cell cascade model at three locations. There are 12 wasteforms, each one meter in length separated by one meter of soil beginning at $\mathrm{x}=0$. The container failure time differs for the wasteforms as described in Table 5.2.

Release and transport parameters can also be found in Table 5.2.

Figure 7.1 Plot of concentration versus time 3 and $23 \mathrm{~cm}$ beneath the last wasteform for the test problems in Table 7.1 (FD) and Table 7.2 (MCMC).

Figure 9.1 Typical graph created by the program GRAFXT . $9-5$ 


\section{LIST OF TABLES}

$\underline{\text { PAGE }}$

Table 2.1 Model Selection Summary................................................................. 2-20

Table 5.1 Parameters used to verify the finite difference transport model ....................... 5-2

Table 5.2 Parameters used in test case 4: Comparison of the mixing cell and finite difference model results ...................................................... 5-6

Table 7.1 Sample finite difference input deck for a single material with containers failing at either 5 or 6 years. Release mechanisms include rinse and uniform dissolution. Blank cards are denoted by the word BLANK in columns $1-5$.....

Table 7.2 Sample multi-cell mixing cascade input deck for a single material with containers failing at either 5 or 6 years. Release mechanisms include rinse and uniform dissolution. Blank cards are denoted by the word BLANK in columns $1-5$.

Table $7.3 \quad$ Input cards required to specify an external source .................................. 7-28

Table 8.1 Typical primary output file when the FD transport model is used ................... 8-2

Table $8.2 \quad$ Typical primary output during the computation when the MCMC transport model is used .............................................................. 8-10

Table $8.3 \quad$ Typical concentration trace file, TRACECND.DAT ................................... 8-12

Table $8.4 \quad$ Typical flux trace file, TRACEFXD.DAT ................................................. 8-14

Table 8.5 Typical concentration versus location file at fixed times, CONCNT.DAT...... 8-15

Table 8.6 Typical wasteform release output file, LEACHRL.DAT ............................ 8-17 


\section{EXECUTIVE SUMMARY}

The U.S. Nuclear Regulatory Commission (NRC) Regulation 10 CFR Part 61, "Licensing Requirements for Land Disposal of Radioactive Waste," requires that after disposal of low-level wastes (LLW) there is reasonable assurance that the general public will not receive annual off-site doses in excess of 25 millirems to the whole body, 75 millirems to the thyroid, and 25 millirems to any other organ.

Demonstration that these regulatory limits are not exceeded requires the quantitative assessment of the potential radiological impact of a LLW disposal facility on the surrounding environment. Evaluation of these impacts is accomplished through a performance assessment which includes estimates of the following processes for each radionuclide: (a) the rate of release from the disposal unit (i.e., the source term); (b) the transport from the disposal unit to the accessible environment; and (c) the conversion of the radionuclide concentration at the receptor site into an equivalent dose.

The objective of this project is to provide a computer model that estimates the radionuclide release rate from the disposal facility, that is, the source term. General guidelines used while developing the computer model included: use of a modular structure to allow further refinements, limiting the complexity of the models to permit the code to be capable of running quickly on a desktop computer system, and including the flexibility to handle a wide variety of situations typically encountered in LLW disposal.

In general, the source term is influenced by the radionuclide inventory and its origin (i.e., waste stream), the wasteforms and containers used to dispose of the inventory, and the physical processes that lead to release from the facility. The complexity of the problem and the absence of appropriate data prevent development of an entirely mechanistic representation of radionuclide release from a disposal facility. Typically, a number of assumptions, based on knowledge of the disposal system, are used to simplify the problem. The assumptions used while selecting the models to represent radionuclide release from the disposal facility and the rationale for these assumptions was presented in a previous report in this program [Sullivan, 1991a].

The models selected to represent the four major processes (fluid flow, container degradation, wasteform leaching, and radionuclide transport) influencing release have been incorporated into the

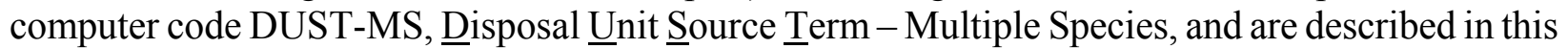
report.

The DUST-MS code calculates the transport of radionuclides through the facility using a one-dimensional finite difference (FD) model. The FD model solves the transport equation with the processes of advection, dispersion, retardation, radioactive decay and ingrowth and external sources (e.g. wasteform releases). The FD splits the modeled domain into a series of control volumes over which contaminant transport is calculated. The model permits temporally changing flow-rates and 
different material properties (sorption, dispersion, porosity, and diffusion) in the subsurface porous media.

The DUST-MS code models fluid flow through tabular input of the flow velocity versus time.

In DUST-MS container degradation is modeled with a unique time to failure for each container. In addition, partial container failure can be modeled. In the partial (localized) failure model, a fraction of the container becomes breached prior to total failure. This permits an earlier release of contaminants from the wasteform.

A specialized version of DUST-MS has also been developed. This version, DUSTMS-D (the $\mathrm{D}$ represents distributed container failure rates) permits a distribution of container failure times. Container degradation is modeled through one of three failure rates: a) instantaneous (all containers in a control volume fail at once), b) uniformly distributed failures (containers fail at a linear rate between a specified starting and ending time), and c) gaussian failure rates (containers fail at a rate determined by a mean failure time, standard deviation and a gaussian distribution). A unique set of container failure parameters can be specified for each control volume with a container.

Wasteform release is modeled through four release mechanisms: a) a surface rinse process in which radionuclides are released upon contact with the solution, partitioning (sorption) between the wasteform and solution can be modeled; b) diffusion controlled release from the wasteform (cylindrical, spherical, and rectangular geometries can be modeled); and the dissolution release model in which a fixed fraction of the inventory is released every year representing dissolution of the surface of the wasteform.. All of these release mechanisms account for radioactive decay and ingrowth of the source. The fourth model is the solubility limited release model. Release predicted by the three other mechanisms are checked to insure that th total release does not cause concentrations to exceed a user defined solubility limit. Unique wasteform release parameters can be specified for up to 20 wasteforms.

For distributed container failures (DUSTMS-D) the release from the wasteform is calculated using a convolution integral that combines the container failure rate with the time-dependent wasteform release rates. The wasteform release models, however, are independent of the container failure models. Conceptually, the distributed container failure model can be viewed as having a series of containers represented in the computational cell. Each of these containers fail at a different time and therefore have different release rates. The wasteform release rate models are used to calculate the release as a function of time and this is combined with the container failure rate to estimate the total release rate from a disposal cell.

Although DUST-MS simulates one-dimensional transport of contaminants often the analyst desires to simulate the flow through the unsaturated zone to an aquifer that typically flows in a direction perpendicular to unsaturated zone flow. This can be analyzed using DUST-MS through two separate simulations. To facilitate this process, DUST-MS can be used to generate an output file of mass flow versus time at a specified location. The output file can automatically be used as 
the upstream boundary condition in the second simulation. This approach conserves mass in the two simulations. The use of this model is discussed in this report.

To ease the burden of creating an input deck a pre-processor, DUSTINMS, was written. DUSTINMS is a menu driven program that guides the user through all of the necessary steps of creating an input deck. Use of DUSTIN permits the user to create an input deck without knowing the formatted structure used by the DUST-MS code. A complete description of DUSTINMS and its operation is provided in this report. To allow direct modification of an input deck, a complete description of the formatted structure of a DUST-MS input deck is also provided. DUSTINMS is a DOS based program.

To facilitate the analysis of the output from the code, the program GRAFMS was written. GRAFMS is a menu driven program that reads files created by DUST-MS and plots the data on a video display unit. Graphs of the concentration, flux, and total mass that has passed through a region can be displayed as a function of time or spatial location.

A Windows based version of the DUSTINMS program named DUSTWIN has also been developed. DUSTWIN allows the user to modify/create input files, run either DUST-MS or DUST, and graph or view output files. DUSTWIN has versions compatible with Windows 3.1 and Windows 95 and 98. A complete description of DUSTWIN and its operation is also provided in Appendix B to the report.

The DUST-MS code has received extensive testing and verification. DUST-MS code predictions have been compared to known analytical solutions as well as other code predictions. A discussion of several of these verification tests is provided.

The DUST-MS code improves upon existing models in that more flexibility is allowed in modeling the various waste stream/wasteform/container systems while still retaining relatively simple models that do not require extensive computer time or provide an undue burden on the code user in terms of input requirements. However, the models used within the DUST-MS code are based on a number of assumptions. A complete discussion of the limitations that result from the assumptions is presented.

In using DUST-MS, as with all computer models, the validity of the predictions relies heavily on the validity of the input parameters. Often, the largest uncertainties arise from uncertainty in the input parameters. Therefore, it is crucial to document and support the use of these parameters.

The DUST-MS code, because of its ability to compute release rates quickly, is extremely useful for screening to determine the radionuclides released at the highest rate, parameter sensitivity analyses, and, with proper choice of the input parameters, provide upper bounds to release rates. 


\section{INTRODUCTION}

The U.S. Nuclear Regulatory Commission (NRC) issued the regulation 10 CFR Part 61, "Licensing Requirements for Land Disposal of Radioactive Waste" [FR, 1982]. 10 CFR Part 61 requires, in part, that any "proposed disposal site, disposal facility design, land disposal facility operations (including equipment, facilities and procedures), disposal site closure, and postclosure institutional control are adequate to protect the public health and safety..." Protection of the public is judged by requiring that releases which may occur must be demonstrated with reasonable assurance not to exceed an equivalent dose of " 25 millirem to the whole body, 75 millirems to the thyroid, and 25 to any other organ of any member of the public." This requires the quantitative assessment of the potential impacts of a low-level radioactive waste (LLW) disposal facility on the surrounding environment. In particular, estimation of the dose to the maximally exposed individual is required.

Estimation of the dose to man is accomplished through a performance assessment. A proposed strategy for conducting such an assessment has been presented by the NRC [Starmer, 1988]. Further guidance will be provided by the NRC in their Branch Technical Position on Performance Assessment [NRC, 1997]. In the proposed performance assessment strategy, performance assessments are conducted through combining a series of separate calculations. These calculations include estimating the rate of radionuclide release from a disposal unit, (i.e., the source term), the transport of the radionuclide from the disposal unit to the accessible environment, and, finally, the conversion of the radionuclide concentration at the receptor site into an equivalent dose. Examples of this approach can be found in the reports produced for the NRC by the staff at Sandia National Laboratory [Kozak, 1989; Kozak, 1990: Chu, 1991].

The objective of this project is to provide computer models that estimate the radionuclide release rate from the disposal facility. A disposal facility is a complex, heterogeneous collection of wastes/wasteforms/containers, soils, and engineered structures (concrete vaults, backfill, vault covers, drains, etc.). For most radionuclides, release from this disposal unit is controlled by access of water to the wasteform, release from the wasteform, and transport to the disposal unit boundary. These processes are influenced by the design of the disposal unit, hydrology, geochemistry and wasteform/container characteristics. A disposal unit is schematically depicted in Fig. 1.1 and typically contains a multi-layered cover to divert water away from the waste; an engineered barrier to further reduce water flow to the wastes (for trench disposal there is no engineered barrier); and metallic, concrete, or HDPE waste containers. The waste comes in many forms including solidified in cement, dewatered resins, activated metals, dry solids (e.g. contaminated paper, cloth, rubber, plastic, glass, etc.).

The heterogeneity and complexity of the disposal facility make development of a threedimensional time-dependent model that covers all possible physical and chemical conditions an extremely cumbersome and difficult task. Even if one could develop models for all conditions, their applicability would be restricted by data limitations and the extensive computer time that would be needed. 


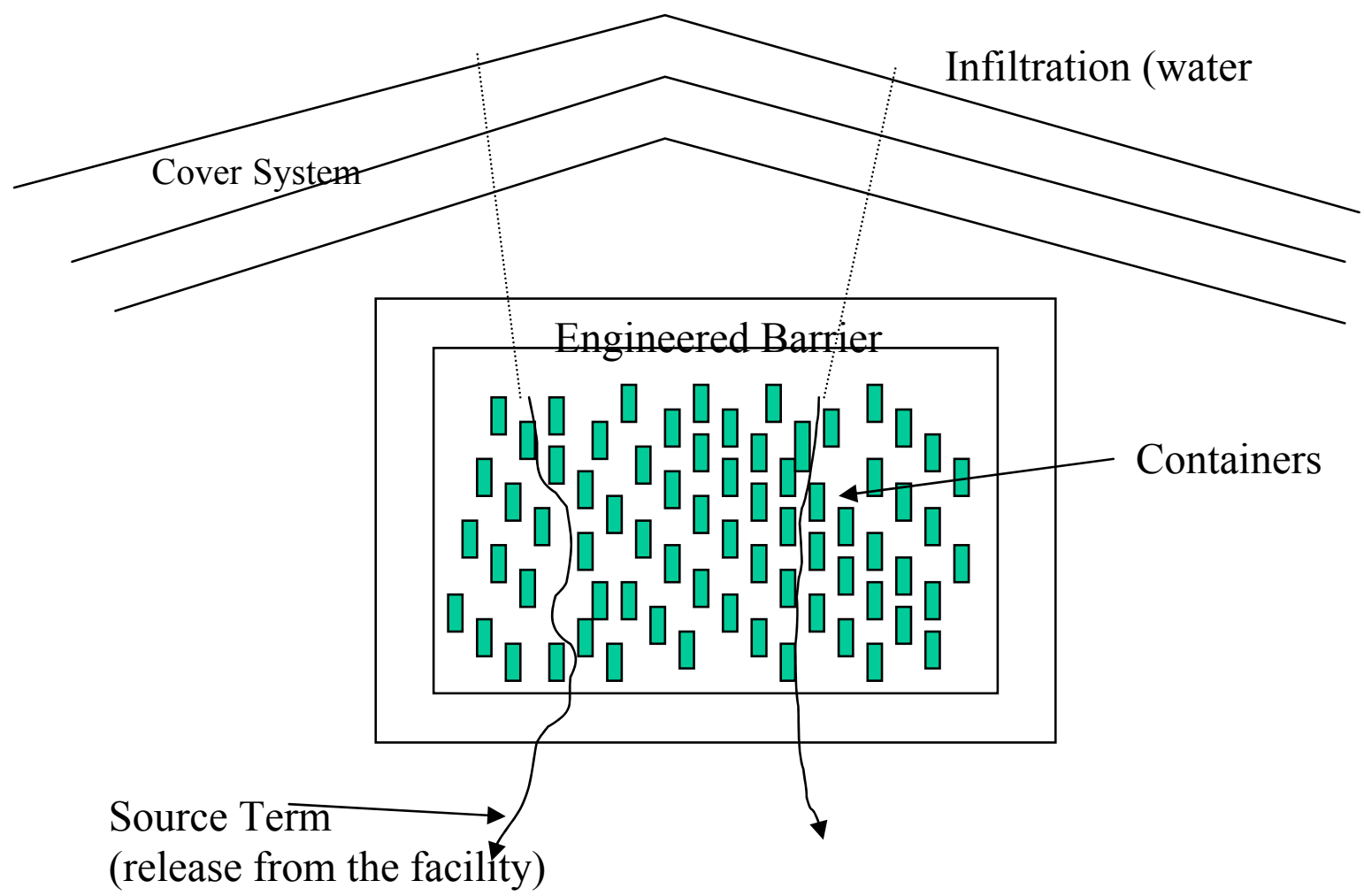

Figure 1.1 Schematic diagram of a LLW Disposal Unit.

Therefore, simplifications are justified. The simplified models should account for the most important physical processes and parameters influencing release while retaining as much accuracy as possible. Further, the models should be flexible enough to simulate the wide range of anticipated conditions and not be overly conservative.

Previous reports, [Cowgill, 1992; Cowgill, 1992a, Duguid, 1995, Yim, 1995], presented a review of waste disposal practices, physical and chemical processes that influence release from the facility, reviewed other source term modeling efforts, and recommended models for incorporation into a source term computer model [Sullivan, 1991b]. These models have been embodied into the computer code DUST-MS, Disposal Unit Source Term-Multiple Species. In addition, a specialized version of the code, DUSTMS-D (the D stands for distributed container failure) has been developed.

Chapter 2 provides a description of the mathematical models and governing equations used to represent water flow, container degradation including distributed failure models and their impacts on release, wasteform leaching including decay and ingrowth, and contaminant transport. A discussion of the range of the parameters in the various models is also provided. 
Chapter 3 discusses the procedure used to operate the DUST-MS code. In addition to DUST-MS, a DOS based pre-processor, DUSTINMS, and Windows based pre-processor DUSTWIN were written to assist the code user in creating an input deck. A post-processor, GRAFMS, was written to take output files generated by DUST-MS and plot them on the video display unit. The DUSTWIN program allows the user to create/modify input files, run the DUST-MS code, view input and output files and plot the results using GRAFMS. The DUSTWIN program is currently not able to create DUSTMS-D input files, but it can run perform all other functions for DUSTMS-D files.

Chapter 4 discusses the limitations of the models.

Several test problems with known analytical solutions have been simulated with the DUSTMS code. These tests have examined the transport model, the wasteform models including ingrowth, and the distributed failure models. The results of these verification tests are presented in Chapter 5.

Chapter 6 presents detailed instructions on how to use the pre-processor DUSTINMS. This code is menu driven and asks the user for all of the variables needed to run DUST-MS. DUSTINMS will take this information and write it to a file in the form required by DUST-MS, thereby, eliminating the need to know the exact structure of an input file. This chapter also discusses the input parameters and provides guidance on their selection. The DUSTWIN pre-processor is similar to DUSTINMS. The use of DUSTWIN and its features ae presented in Appendix B.

Chapter 7 discusses the format of the input required by DUST-MS. This permits the user to create or modify an input deck without using DUSTINMS. In addition, the modifications required to transform a DUST-MS iinput file into a DUSTMS-D input file are discussed in Chapter 6 and 7. Sample input decks are provided and discussed.

Chapter 8 discusses the various output files created by DUST-MS. In addition to the main output file, which contains the problem definition and the requested output (concentrations, fluxes, and wasteform mass release rates), DUST creates files containing concentration, flux, or total mass that has moved past a given location for use with GRAFMS, and files containing information on the wasteform release rates.

Chapter 9 discusses the use of GRAFMST, the graphics program for DUST-MS output files, TRACECND.DAT, TRACEFXD.DAT, and CONCNT.DAT.

Chapter 10 provides a brief summary and conclusion for this report. 


\section{MODEL SELECTION AND GOVERNING EQUATIONS}

A LLW disposal unit is a complex, heterogeneous collection of wastes/ wasteforms/containers, soils, and engineered structures (clay caps, concrete vaults, drains, etc.). Aqueous release of radionuclides from this disposal unit is controlled by water flow, access of the water to the wasteform, release of the radionuclide from the wasteform, and transport to the disposal unit boundary. These processes are influenced by the design of the disposal unit, precipitation, hydrology, geochemistry, and wasteform/container characteristics. To model the complete disposal unit, including every waste container individually would require a three dimensional model that considered all of these processes simultaneously. Such a model does not exist today. Even if such a model did exist, its use would require extensive computing times and the accuracy of the predictions would be questionable due to limitations in the data.

Therefore, simplifications from a fully descriptive three-dimensional model are justified. These "simplified" models are a necessary step in developing predictions of the behavior of a LLW disposal site.

The "simplified" models incorporated into DUST-MS account for the most important physical processes and parameters influencing release. Further, the models are flexible enough to simulate a wide range of conditions. For example, multiple container failure and wasteform release rates can be modeled.

The DUST-MS code has been developed in a general manner which allows simulation of the majority of situations expected to occur. However, to account for the possibility of special cases and allow easy modifications of the models within the code to reflect new and better information, the code structure is modular.

In the following sections, the framework for the DUST-MS code is presented. Within that framework, the models for the four processes (water flow, container degradation, wasteform release, and transport) that influence release are discussed separately. This chapter contains the basic equations that govern the release and transport within the facility However, the detailed equations describing the analytical solutions used in modeling these processes are presented in Appendix A.

\subsection{DUST-MS Code Framework}

The ultimate objective of the DUST-MS code is to predict the rate of release of radionuclides from a shallow land disposal facility. As discussed earlier, this is accomplished through use of computer models. These models should be simple enough to allow simulation of a large number of cases and flexible enough to allow simulation of a wide range of situations. To achieve this one can use analytical or numerical solution procedures. Analytical solutions often have the advantage of being easier to compute than numerical solutions. Numerical solutions offer the flexibility to model a wider range of conditions. Due to the complexity of the problem (multiple species, multiple 
containers and wasteforms), numerical solutions are performed in DUST-MS. An analytical solution procedure is provided in the DUST (Sullivan, 1993) code for a single species with multiple containers, however, the transport properties must remain the same throughout the modeled domain.

The DUST-MS code was written in an attempt to achieve a balance between the use of extremely simple but conservative assumptions, which lead to high predicted release rates, and complicated models that include all of the known physical and chemical processes that influence release but require extensive computer time and expertise to define the problem (select the input variables). To strike this balance, assumptions regarding which are the most important physical parameters and the level of detail needed to calculate these parameters have been made. These are discussed in detail in the model selection report [Sullivan, 1991].

The primary equation for predicting release from the disposal facility is the contaminant transport equation. In addition, there are models that supply information on fluid flow, container degradation and wasteform release. A flow chart of the various subroutines that comprise the DUSTMS code is presented in Appendix C.

The transport equation in DUST-MS is solved using a finite difference approach. The finite difference model divides the simulated domain into finite regions called control volumes. The finite difference model permits different transport properties in each control volume.

The ultimate objective of the DUST-MS code is to predict the transport of the radionuclides out of the facility. The wasteform release models provide a source to the transport equation. Therefore, the transport model is presented first.

\subsection{Radionuclide Transport}

The major pathway for release of most radionuclides disposed in a low-level waste disposal facility is movement with the groundwater. Movement of subsurface contaminants is influenced by the processes of groundwater flow, dispersion, diffusion, radioactive production and decay, geochemistry (solubility and sorption) and sources and sinks (waste form releases). The relative importance of these processes is site- and contaminant-specific.

In DUST-MS, after the completion of the waste form release calculations, the movement of the radionuclides through the subsurface system is calculated using a one-dimensional finite difference approximation to simulate transport.

The governing transport equation that is solved in DUST-MS is derived from a mass balance over the solid and liquid phases. The solid phase is treated using the assumption that it is immobile and the aqueous concentration is in chemical equilibrium with the solid phase concentration. The constitutive relationship between the mass adsorbed on the solid phase and that in the liquid phase is known as the sorption isotherm. DUST-MS uses a linear sorption isotherm. Water flow and moisture contents within the modeled domain are supplied through tabular input as described in 
section 6 and 7. The governing equation simulates the distribution and movement of contaminants due to advection, dispersion, diffusion, radioactive production and decay, sorption, and external sources and sinks (i.e., waste forms as well as other sources).

After obtaining the waste form mass release rates per unit volume for each container and each contaminant, DUST-MS takes this information and solves for the movement of each contaminant individually.

\subsubsection{Transport Equation with Chain Decay}

The governing transport equation is:

$$
\theta R_{i} \frac{\partial C_{i}}{\partial t}=\nabla \bullet \theta D_{i} \nabla C_{i}+\nabla \bullet V_{d} C_{i}-\lambda_{i} \theta R_{i} C_{i}+\sum_{j=l-N}^{j i} f_{i j} \lambda_{j} R_{j} C_{j}+S_{i}
$$

where in one-dimension the dispersion diffusion term is:

$$
\theta D=a_{t} V_{d}+D_{m} \theta
$$

and: $\quad \mathrm{C}_{\mathrm{i}}$ is the concentration of the $\mathrm{i}^{\text {th }}$ contaminant in the aqueous phase $\left(\mathrm{g} / \mathrm{cm}^{3}\right)$, $\mathrm{t}$ is the time (s)

$\theta$ is the volumetric moisture content, $R_{i}$ is the retardation coefficient for contaminant $I$,

$$
\mathrm{R}_{\mathrm{i}}=1+\rho \mathrm{K}_{\mathrm{d}, \mathrm{i}} / \theta
$$

$\rho$ is the bulk density of the soil $\left(\mathrm{g} / \mathrm{cm}^{3}\right)$,

$\mathrm{K}_{\mathrm{d}, \mathrm{i}}$ is the distribution coefficient for $\mathrm{i}^{\text {th }}$ contaminant in the porous media

$\theta \mathrm{D}$ is the component of the dispersion-diffusion tensor $\left(\mathrm{cm}^{2} / \mathrm{s}\right)$,

$\mathrm{a}_{\mathrm{t}}$ is the transverse dispersion coefficient $(\mathrm{cm})$,

$V_{d}$ is the Darcy velocity through the porous medium $(\mathrm{cm} / \mathrm{s})$,

$\mathrm{D}_{\mathrm{m}}$ is the molecular diffusion coefficient corrected for tortuosity through the porous medium $\left(\mathrm{cm}^{2} / \mathrm{s}\right)$,

$\lambda_{\mathrm{i}}$ is the radioactive decay coefficient of contaminant $\mathrm{I}\left(\mathrm{s}^{-1}\right)$,

$f_{i j}$ is the fraction of decays of contaminant I that form contaminant $j$,

$\mathrm{N}$ is the number of contaminants in the simulation,

$S_{i}$ is the external source injection rate per unit volume which includes release from the waste form. $\left(\mathrm{g} / \mathrm{cm}^{3}-\mathrm{s}\right)$.

Although not explicitly addressed in the above equation, many of the above parameters can also vary spatially due to local differences in the physical and chemical properties of the porous 
media. Typically when using DUST-MS to represent variations in transport properties, regions are defined with a unique set of material properties. For example, the cover system could be designated as material 1, the engineered concrete structure as material 2 , the waste containing region as material 3 , and the undisturbed soil surrounding the facility as material 4. Each of these material regions could be assigned unique transport properties. In DUST-MS, the distribution coefficient, diffusion coefficient, dispersion coefficients, and bulk density are allowed to vary with each material and each contaminant. In general, the soil bulk density is independent of the contaminant. The dispersivity term is a mathematical construct that has been shown to be useful in describing transport of contaminants in subsurface systems. Typically contaminant specific dispersion coefficients are not measured.

Equation 2.1 contains a generalized source term, $\mathrm{S}_{\mathrm{i}}$. This term represents all external sources to the transport equation. DUST-MS considers two distinct types of sources, waste form sources and volumetric sources. The values for the waste form source are calculated in accordance with the expressions provided in Section 2.5. Volumetric sources are specified through tabular input of the source strength versus time as described in section 6.11. Volumetric sources are distributed over the volume of the computational cell at which they are injected. In addition, due to the implicit treatment of rinse with partitioning, an additional term is added to the transport equation to represent the source due to equilibrium partitioning between the waste form and aqueous solution. As the solution concentration changes over time, mass will be released (or sorbed) by the waste form to maintain equilibrium.. This relationship is:

$$
S_{i}=\frac{\partial\left[\rho_{w f} S_{i, r, w f}\right]}{\partial t}+S_{i, w f}+S_{i, e x t}
$$

where $\mathrm{S}_{\mathrm{i}, \mathrm{wf}}$ is the waste form source due to diffusion and uniform degradation processes, $\mathrm{S}_{\mathrm{i} \text {,ext }}$ is the external source term, and the first term represents the source required to maintain equilibrium between the waste form and solution when partitioning occurs. When distributed container failures are modeled (DUSTMS-D), the waste form source term is modified to account for the distribution of failures. This is discussed further in section 2.5.X of this report.

Assuming equilibrium sorption, the first term becomes:

$$
\frac{\partial\left[\rho_{w f} S_{i, r, w f}\right]}{\partial t}=\partial \frac{\frac{\left[\rho_{w f} K_{p} V_{w f} C\right]}{V s u b e}}{\partial t}-\lambda \frac{\rho_{w f} K_{p} V_{w f}}{V_{e}} C
$$

Placing Eqn.2.4 into the transport equation, Eqn. 2-1, gives the transport equation solved in DUST-MS. 


$$
\theta R_{i^{\prime}} \frac{\partial C_{i}}{\partial t}=\nabla \bullet \theta D_{i} \nabla C_{i}+\nabla \bullet V_{d} C_{i}-\lambda_{i} \theta R_{i^{\prime}} C_{i}+\sum_{j=I-N}^{j_{i} i} f_{i j} \lambda_{j} R_{j^{\prime}} C_{j}+S_{i, w f}+S_{i, e x t}
$$

Here, the retardation coefficient on the time derivative, decay term, and ingrowth term all have been modified to account for sorption on the waste form. The modified retardation coefficient is:

$$
R_{i^{\prime}}=1+\frac{\rho K_{d, i}}{\theta}+\frac{\rho K_{p^{\prime}, i}}{\theta}
$$

where $K_{p, i}$ is the modified waste form partition coefficient. This will be discussed further in the rinse release model section of this report, Section 2.5.1.

\subsection{Fluid Flow}

Although setting a disposal facility beneath the water table is permitted, it is expected that new facilities will be located above the water table in the unsaturated zone. In either case, the most likely pathway for release will be through the water. Infiltration of water into a facility will involve many processes including precipitation, evapotranspiration, and surface run-off. Water flow in the unsaturated zone is difficult to predict due to the non-linearity of the unsaturated soil flow properties. This is further complicated by the barriers (trench cap, concrete structure, etc) any disposal facility will have to minimize infiltration into the waste containing region.

A simple model for infiltration is needed. In addition, predicting infiltration into soils in arid sites under all conditions is difficult [Gee, 1988]. To calculate flow into a disposal facility would require at least a two-dimensional simulation. Further, the flow rate will vary with time on a short time scale (hours) due to precipitation events and evapotranspiration and on a long time scale (years) due to changes caused by degradation of the infiltration barrier. To follow the evolution of water flow with time would require an extensive computing expense. Thus, this is not appropriate for the source term model.

In the DUST-MS code water infiltration is calculated as a function of time through tabular input.

$$
V_{d}(t)=F(t)
$$

where $V_{d}$ is the volumetric flow velocity (Darcy velocity) of the water and $F(t)$ is defined in an input table as discussed in Chapter 6 and 7.

This flow rate should be the yearly average based on the expected conditions. For advection driven transport it has been shown that the average rate of contaminant transport depends on the average flow rate [Sullivan, 1988a].

The choice of the value for the flow rate should be conservatively chosen or supported by 
more detailed computer simulations such as VAM2D [Huyakorn, 1989], FEMWATER [Yeh, 1987], TRACR3D [Travis, 1991], etc. If a computer simulation is not performed, an upper bound for the flow rate is the annual precipitation rate. If the evapotranspiration rate is accurately known this could be subtracted from the precipitation rate at humid sites. At arid sites this may lead to large errors in predicted recharge [Gee, 1988]. Alternatively, if the recharge rate through the disposal facility is known due to measurement at the site, this value could be used.

In the actual situation, infiltration may be very low until significant degradation of the cap occurs. If one accounts for degradation of the cap, this will require additional modeling. At this time, there is no widely accepted model for the degradation of earthen materials or engineered (i.e., concrete) caps. This is due in part to the need to predict performance over hundreds of years based on experience and data that have been collected over a period of years.

However, work is being performed to determine the degradation mechanisms of underground concrete structures. Models based on these studies are under development [Clifton, 1989; Walton, 1990; Shuman, 1991] and should help in estimating the rate of degradation. This information could then be used to calculate water flow through the degraded barrier and into the waste containing region of the disposal facility.

\subsubsection{Gas Flow}

If it is determined that the gaseous pathway may be significant, the flow of gas through the facility will need to be modeled. Conceptually, the DUST-MS code would handle the problem in an identical fashion to that for water flow, i.e., a table of volumetric gas flow rate versus time could be used for the transport model. Models for the production and/or release of gaseous phase radionuclides would have to be developed.

The transport of gaseous radionuclides within the disposal facility requires special attention. For gases, flow may be up and out of the disposal facility or out of the bottom through drains. Upward migration will involve advection due to pressure variations that will vary seasonally and diurnally, as well as diffusion. Detailed assessments of gas phase release have been performed by Yim [Yim, 1995]. This work focused on release of C-14 and radon from subsurface disposal facilities..

As with water flow, prediction of the upward migration of gas is a complicated problem. Due to the length of time to be considered in a performance assessment, it is impractical to model daily or seasonal variations in gas flow rate. Therefore, it will be necessary for the code user to supply an average gas advection velocity in order to calculate gaseous release. This flow rate should be estimated using state-of-the-art computer codes or, as a minimum, chosen to permit conservative predictions of gas release. 


\subsection{Container Degradation}

Waste containers in the early days of LLW disposal ranged from cardboard and wooden boxes to carbon steel drums and boxes. Since the passage of 10 CFR Part 61, cardboard and wooden boxes are no longer used. As of 1988, carbon steel drums and boxes were widely used to dispose of Class A wastes, the largest volume of wastes. Most Class B and C wastes are disposed of in highintegrity containers (HIC) but a small fraction have been stabilized in cement and placed in 55 gallon drums [Sullivan, 1989]. Recently, there has been a trend to rely more and more exclusively on HIC's for Class B and C wastes due to their ease of use, lack of need for processing equipment, reduced worker exposure, problems encountered with solidification of some waste streams, and their approval by NRC as a means of demonstrating structural stability.

A waste generator has a number of different HIC's from which to choose. These include HIC's made from Ferralium 255, from stainless steels, from polymer-impregnated concrete, and high-density polyethylene (HDPE). A HIC may also have an internal lining to isolate the waste from the external barrier to water flow. The liner materials are typically polyethylene. HIC's should be designed to maintain their structural stability and maintain a positive seal for 300 years as indicated in the NRC Technical Position on Wasteform [Higginbotham, 1983; Lohaus, 1991]. Structural stability does not imply that the HIC's will remain water tight. In time, water may enter through the passive gas vents required on HIC's or through small cracks and localized failures that may occur.

Currently, most waste containers are metallic. The use of HDPE alone is suspect due to the potential of long term creep affecting its stability and it is no longer on the NRC-approved list of HIC's. HDPE is used as a liner in metallic containers and within a concrete caisson which is backfilled with soil. The Richland site received five of these concrete caisson/HDPE HIC's in 1988 [Sullivan, 1989].

Modeling of metallic corrosion on a mechanistic scale is strongly dependent on the local chemistry and quite complicated. For the source term model, the work required to perform such a calculation is not justified. Rather, in the DUST code metallic container degradation models will be semi-empirical and rely on the existing corrosion in soil data base. If internal corrosion is expected to be important, this can also be included in the empirical model, however, data in this area is lacking. In the DUST code, two types of failure are modeled: general failure, and localized failure.

\subsubsection{General Failure}

In the DUST code general failure is modeled through a user-specified time of failure. In this model, the container prevents water ingress to the waste until failure, at which time the container no longer provides a barrier to water flow. For metallic containers, the time to failure could be estimated as the thickness of the container divided by the time-averaged corrosion rate.

Corrosion rates should be obtained from site specific data whenever possible. When this is not possible, the data base generated by the National Bureau of Standards, NBS (currently, National 
Institute for Standards and Technology) [Romanoff, 1957; Gerhold, 1981] for carbon steels and stainless steels could be used for these materials. There is no data base for the corrosion of Ferralium in soil systems. However, Ferralium, a duplex stainless steel, has shown superior corrosion performance as compared to 304 and 316 stainless steels in a wide range of environments. If this trend holds for soil systems, use of the NBS data for stainless steels should be conservative.

The NBS studies of carbon steels covered a period of 17 years and 47 different soils [Romanoff, 1957]. Uniform corrosion rates in this study of carbon steels ranged from $8 \times 10^{-4}-2 \times 10^{-}$ ${ }^{2} \mathrm{~cm} / \mathrm{yr}$, with the mean value being $5.7 \times 10^{-3} \mathrm{~cm} / \mathrm{yr}$. In the LLW Updated Impacts Analysis [Otzunali, 1986] the recommended value for carbon steel corrosion was 4 mils $/ \mathrm{yr}\left(1 \times 10^{-2} \mathrm{~cm} / \mathrm{yr}\right)$. For typical 55 gallon carbon steel drum thicknesses, 50 mil, container lifetimes would be expected to range from 6 to 160 years with a mean lifetime of 23 years based on the above data.

The NBS studies of 304 and 316 stainless steels were conducted over 14 years in 15 soils. General corrosion rates for 304 stainless steel ranged from $1.7 \times 10^{-5}-1.1 \times 10^{-7} \mathrm{~cm} / \mathrm{yr}$, with the mean value being $5 \times 10^{-6} \mathrm{~cm} / \mathrm{yr}$ [Gerhold, 1981]. Corrosion rates for 316 stainless steel ranged from $5.7 \times 10^{-6}-2.8 \times 10^{-8} \mathrm{~cm} / \mathrm{yr}$, with the mean value being $1.3 \times 10^{-7} \mathrm{~cm} / \mathrm{yr}$ [Gerhold, 1981]. Otzunali recommended a value of $0.3 \mathrm{mils} / \mathrm{yr}\left(7.6 \times 10^{-4} \mathrm{~cm} / \mathrm{yr}\right)$ [Otzunali, 1986]. For a $3 / 8$ inch thick stainless steel container, the lifetime based on the corrosion rate recommended by Otzunali would be 1250 years.

In the soil corrosion experiments, it was noted that corrosion rates typically decreased over time [Romanoff, 1957, Gerhold, 1981]. Provided that there is no change in the degradation mechanism, the experimentally measured decreasing corrosion rate indicates a constant rate based on short term data is likely to overpredict the total amount of corrosion.

For containers with non-metallic components (HDPE containers, polyethylene lining in metal, concrete caissons, etc.) there are few data on their long term performance. It is recommended that the general failure rate be selected in a conservative manner based on expert judgement.

\subsubsection{Localized Failure}

If localized failure occurs, water will contact the wasteform causing the release of radioactivity before the general corrosion allowance is reached. This solute may be released immediately after local failure or it may be stored within the container causing a large pulse type release when general failure occurs. In either event it may have a significant impact on predicted releases from the disposal facility.

For metallic HIC's localized failure can occur due to pitting, stress corrosion cracking, failure of the passive gas vents, or other mechanisms. In these failure scenarios, only a small portion of the container will permit water access to the wasteform. The reduced water flow impacts on the amount released and available for transport. This is accounted for in the leaching model. 


$$
A_{b}=N_{p} A_{c}\left(h^{2}-T^{2}\right)
$$

where $\quad \mathrm{Np}$ is the number of localized failures per unit area of the container;

Ac is the total container area;

$\mathrm{T}$ is the thickness of the metal; and

$\mathrm{h}$ is the penetration depth.

The penetration depth is estimated from

$$
h=k t^{n}
$$

where $\mathrm{t}$ is time in years, and $\mathrm{k}$ and $\mathrm{n}$ have been determined for carbon steel [Mughabghab, 1988] based on the NBS corrosion data in soils [Romanoff, 1957; Gerhold, 1981]. For carbon steel, the parameter $\mathrm{k}$ was found to depend on the soil-water $\mathrm{pH}$ and the parameter $\mathrm{n}$ depends on the degree of soil aeration, moisture content, and clay content. The value for $\mathrm{n}$ is always less than 1 and is higher for poorly aerated soils (poor drainage) as compared to well aerated soils.

A detailed discussion of the choice of values for $\mathrm{N}_{\mathrm{p}}, \mathrm{k}$ and $\mathrm{n}$ can be found in the BLT data input guides [Sullivan, 1989], which is reproduced in Appendix D. For carbon steels, $\mathrm{N}_{\mathrm{p}}$ was found to range from 0.05 to 0.5 per $\mathrm{cm}^{2}$, the average value for $\mathrm{k}$ was $0.0457 \mathrm{~cm} / \mathrm{yr}^{\mathrm{n}}$ and $\mathrm{n}$ ranged from 0.01 to 0.93. Typical values for these parameters are discussed in Section 6.8.6.

For stainless steels, even though there are 14 years of corrosion data in 15 different soils [Romanoff, 1957; Gerhold, 1981], the data are insufficient to support estimation of the necessary parameters. Thus, for these and other container materials, the parameters will have to be estimated using engineering judgement if localized corrosion is modeled.

Through proper choice of the localized corrosion parameters, failure of the passive gas vents required on HIC's may also be modeled. For example, by setting $n$ to zero and appropriate choice of the parameters $h$ and $N_{p}$, a constant area of failure may be estimated. This failure area may be particularly important if gaseous release is being modeled.

Consideration should also be given to internal corrosion. HIC's may store wastes without the waste undergoing a solidification process. In this case, the wastes may directly contact the lining or, in the absence of a lining, the container material. Many LLW wastes contain corrosive agents that could possibly lead to penetration via pitting (localized failure). For example, it has been shown that resin beads in contact with stainless steel led to discoloration and pitting in short term tests [Soo, 1990].

\subsubsection{Distributed Container Failure Models (DUSTMS-D code)}

DUST-MS is a one-dimensional (1-D) model that predicts the release and transport of contaminants disposed in the subsurface. The conceptual model collapses the 3-D physical system 
down to 1-D mathematical representation. This implies that there are frequently multiple containers represented in one computational cell by a single effective container. This effective container can fail at a specified time that represents the mean time to failure of all containers represented in the computational volume. However, in practice it is probable that the containers will fail over a distribution of times. To account for this, the single failure time is generalized to a distribution of failure times. In theory, the distribution can be any function. In most cases, the distribution of failure time approximates known statistical distribution functions such as the uniform, normal, lognormal, or exponential distribution functions. A specialized version of the code, DUSTMS-D, that permits time-distributed container failure rates for each cell with a container has been developed. The second D in DUSTMS-D refers to distributed failures. DUSTMS-D is an extension of DUST-MS and has all of the capabilities of DUST-MS. The changes icorporated in DUSTMS-D include:

a) Allowing a unique burial time for each container. In practice, a disposal site may be open for many years. Inventory values are reported at the time of disposal. The improved model permits a user to specify a problem start time (i.e., time at which waste was first disposed) and a disposal time for each container. This improves the accuracy for calculating releases radionuclides that have a half-life on the order of the operational time of the facility or less.

b) Allowing time-distributed container failures. In previous versions of the model, container degradation was modeled through a unique container failure time. The value for this parameter should be selected based on the materials and expected environment. It was recognized that in using the one-dimensional DUST-MS code a single modeled container often represents a series of containers. In practice the failure time of each container in the series will be different. To accommodate this, DUST-MS was generalized to permit a distribution of container failures. The distribution will be specified using either a uniform failure rate or a Gaussian (normal) distribution characterized by a mean and standard deviation.

c) Allowing a fraction of the containers to fail on emplacement. Experience has indicated that often a small fraction of the containers fail either due to emplacement practices or soon after emplacement. The improved models in DUST-MS permit the user to specify an initial failure fraction while allowing the remainder to fail based on the selected distribution and input parameters.

In DUSTMS-D three failure distributions are permitted, instantaneous, uniform, or normal.

a) Instantaneous failure of all containers at time $t_{j}$ The failure distribution function, which represents the rate of change in container failures as a function of time, is:

$$
f\left(t-t_{j}\right)=\delta\left(t-t_{j}\right)
$$

where $\delta\left(t-t_{j}\right)$ is the Kronicker delta function. The only information required for this model is the time to failure. 
This is the release rate for a single failure time for all containers and is the model in the previous versions of DUST-MS.

b) Uniform Container Failure Rate

The containers fail at a uniform rate from the beginning time of failure, $t_{b}$, to the ending time of failures, $\mathrm{t}_{\mathrm{e}}$. The failure distribution function is:

$$
\begin{aligned}
f\left(t-t_{j}\right) & =0 & & t<t_{b} \\
& =\frac{1}{\left(t_{e}-t_{b}\right)} & & t_{b} \leq t \leq t_{e} \\
& =0 & & t>t_{e}
\end{aligned}
$$

The information required for this model is the failure start time, $t_{b}$ and the failure finishing time, $t_{e}$.

c) Normal distribution failure rate.

If the container failure rate follows a normal distribution, the distribution function is:

$$
f(t)=\frac{1}{\sigma \sqrt{2 \pi}} e^{-(t-\mu)^{2} /\left(2 \sigma^{2}\right)}
$$

where $\sigma$ is the standard deviation and $\mu$ is the mean value for failure times.

User supplied input controls the choice of failure model. The implementation of the failure models and how they impact release is described in 2.5.10.

\subsection{Wasteform Leaching}

Radionuclide release from the wasteform commences upon container failure. In a LLW facility there will be several different wasteforms, a partial list of which includes: wastes solidified by one of several processes (cement, VES, bitumen); activated metals; compacted lab trash; dewatered resins; liquids contained in an absorbent; and adsorbed gases [Roles, 1990]. The disposal data must be analyzed in order to determine the most likely release mechanisms for each radionuclide. After analyzing the data, each of the major categories of wasteforms should be

grouped in terms of release mechanism. Review of the disposal data shows the following major waste streams:
a) activated metals;
b) dry active wastes;
c) resins; and
d) filter media 
Approximately $70 \%$ of the activity is in activated metals, $15 \%$ of the activity is solidified in cement, and most of the rest is dry active wastes [Sullivan, 1991b]. However, the distribution by radionuclide changes markedly from one radionuclide to the next. For example, over $80 \%$ of the Th232 is disposed of with sorbents, over $50 \%$ of the C-14 is in cement, etc [Cowgill, 1992]. Therefore, the user must determine the distribution on a radionuclide specific basis.

Based on the above groups the following release mechanisms will be modeled:

a) Solubility limited;

b) Surface wash-off subject to partitioning;

c) Diffusion; and

d) Uniform (e.g., Dissolution).

In general, a wasteform may release radionuclides by more than one mechanism. This will be allowed through user-supplied input. In particular, the user will be allowed to specify the fractional amount of mass released by each mechanism. For example, the user could specify that for $10 \%$ of the mass, release is controlled by surface wash-off, while the other $90 \%$ is controlled by diffusion. This flexibility may prove to be important when homogenizing the number of waste streams/wasteforms or in modeling large boxes containing many wasteforms.

\subsection{Waste Form Release Models}

The objective of the waste form release models is to estimate the rate of release of radionuclides from the waste form to the water after the container has failed. The release rate depends upon the physical and chemical form of the radionuclides in the wastes. To simulate the range of waste form conditions, DUST-MS has four process models that can be used to estimate the rate of release: rinse with partitioning, diffusion, uniform degradation, and solubility limited release.

Low-level radioactive wastes are generated from a variety of different processes by the nuclear industry, medical industry, universities, government, and private industry. The variety of processes that generate the waste leads to a wide range in the physical and chemical properties of the wastes. In low-level waste management the wastes can be disposed of in many forms including activated metals, cement solidified wastes, lab trash, evaporator bottoms, ion-exchange resins, and other forms. The U.S. NRC's Uniform Low-level Radioactive Waste Manifest lists over twenty different categories of wastes.[NRC, 1995] Detailed discussions of the distribution of radionuclides by various waste forms can be found in a number of studies [Yim, 1995, Dehmel, 1994, Cowgill, 1993, Roles, 1989].

In obtaining an estimate of release from the waste form, the analyst should first consider all of the different types of wastes and the distribution of radioactive contaminants by waste form. Based on this knowledge of the inventory, the analyst should attempt to define the leaching mechanisms appropriate for each waste type (for example, solidified wastes are often assumed to control release through the diffusion process, activated metals, and waste glass are often treated to 
release contaminants due to dissolution which is modeled as a constant rate process, etc.). In the absence of information, the rinse model, which releases the entire inventory on contact with water, is often used to simulate waste form releases.

The following sections will describe the four process models incorporated into DUST-MS. DUST-MS has two distinct approaches to modeling waste form release. In the first approach, analytical models are used to represent each of the processes. The analytical approach is conceptually easy to understand, but it does not always account for different interactions between release mechanisms. The second approach represents the waste form numerically using finite differences. In this approach, all release mechanisms are calculated simultaneously. In addition, the finite difference approach provides a natural method for handling ingrowth of progeny when diffusion is important.

In both the analytical and finite-difference release models a distinction is made on the mass available for each release process. In DUST-MS, input defines the amount of mass available for each release mechanism. This permits homogenization of wastes into a single waste form. For example, if in some region of the disposal facility $60 \%$ of the activity is in activated metals, $30 \%$ in compacted lab trash, and $10 \%$ in cement solidified wastes, the code user can specify that $60 \%$ of the mass is available for uniform degradation release modeling, 30\% of the mass is available for rinse with partitioning, and $10 \%$ is available for diffusion controlled release. Transfer of mass does not occur between release mechanisms unless the container is partially failed. In this case, mass that would be released in the absence of the constraints will be transferred to the rinse with partitioning model to permit faster release once the release constraints are not limiting. This is discussed in more detail in section 2.5.4.1 (partial container failure) and 2.5.6 (solubility limited release).

It is important for the analyst to realize that within the finite difference framework, the waste form sources act to inject the contaminants at specific places within the modeled domain. Waste form properties are independent of the soil transport properties. This distinction becomes important when simulating rinse release with partitioning and in conceptual model development for waste emplacement in the model. This will be discussed in Section 2.5.1 in more detail.

\subsubsection{Rinse Release with Partitioning}

The conceptual model represented by the rinse release model conforms to one that all of the radioactive contamination is on the surface of the wastes and will be released as soon as water contacts the wastes (e.g., immediately following container failure) subject to equilibrium partitioning and solubility limits. In the absence of partitioning and solubility constraints, an instantaneous release of the entire waste form inventory occurs and the inventory is available for transport.

The more general case occurs when there is partitioning of the mass between the wastes and the contacting porous medium due to sorption. Conceptually, this model considers three media, the waste form, the aqueous solution, and the porous media surrounding the waste form. . 
Initially, the entire inventory is in the waste form and the system is not at equilibrium. After container failure, the mass released from the waste form is calculated to correspond to the amount required such that there is equilibrium between the three components in the system. In theory, this is an instantaneous process. In the implementation in DUST-MS, the mass is released instantly at the beginning of the time step. That is, the rinse mass is calculated, and the solution concentration is immediately adjusted to the equilibrium value. For each radionuclide, the amount of mass released over the time step is obtained by performing a mass balance. Prior to equilibration, the mass in the system is:

$$
M_{i, t}=M_{i, w f}+M_{i, a}+M_{i, p}
$$

where $\mathrm{M}_{\mathrm{i}, \mathrm{t}}$ is the total mass ( $\mathrm{g}$ ) in the system of contaminant I, (i.e., finite difference control volume), $\mathrm{M}_{\mathrm{i}, \mathrm{wf}}$ is the mass of contaminant I on the waste form, $\mathrm{M}_{\mathrm{i}, \mathrm{a}}$ is the mass of contaminant I in aqueous solution, and $\mathrm{M}_{\mathrm{i}, \mathrm{p}}$ is the mass of contaminant I sorbed on the porous media. For notational convenience, the subscript I which denotes contaminant I in the simulation is omitted from the remainder of this section. The different masses are given by the following expressions.

$$
M_{a}(t)=\theta(t) V_{e} C(t)
$$

where $\theta$ is the volumetric moisture content in the system, $\mathrm{V}_{\mathrm{e}}\left(\mathrm{cm}^{3}\right)$ is the volume of the porous media and aqueous solution system, $\mathrm{t}$ is the time (s), and $\mathrm{C}$ is the concentration of the aqueous solution $\left(\mathrm{g} / \mathrm{cm}^{3}\right)$.

$$
M_{p}(t)=\rho S_{p}(t) V_{e}
$$

where $\rho\left(\mathrm{g} / \mathrm{cm}^{3}\right)$ is the bulk density of the porous media, and $\mathrm{S}_{\mathrm{p}}$ is the mass adsorbed on the solid per unit mass of the solid $(\mathrm{g} / \mathrm{g})$. The mass contained in the waste form is the inventory of the waste form at time, t. Expressing the waste form mass in a form similar to the porous media mass yields:

$$
M_{w f}(t)=\rho_{w f} S_{w f}(t) V_{w f}
$$

where the subscript wf refers to the waste form. Notice, the volumes of the waste form and porous media are assumed to be different. This distinction is important when homogenizing regions of waste and soil into a single computational volume. Computationally, the wastes and the soil occupy the same region of the finite difference domain.

The assumption of chemical equilibrium permits a direct relationship between the amount sorbed on the soil (or waste form) and the solution concentration as expressed by:

$$
S_{p}(t)=K_{d} C(t)
$$


and

$$
S_{w f}(t)=K_{p} C(t)
$$

where $K_{d}\left(\mathrm{~cm}^{3} / \mathrm{g}\right)$ is the soil-solution distribution coefficient and $\mathrm{K}_{\mathrm{p}}\left(\mathrm{cm}^{3} / \mathrm{g}\right)$ is the waste formsolution partition coefficient. The partition and distribution coefficients are conceptually and physically similar parameters. For clarity in the report, however, distribution coefficient will be used to discuss the soil-solution chemical equilibrium used in the transport equation, and partition coefficient will strictly apply to the waste form-solution equilibrium coefficient.

Assuming that the soil and solution are always in equilibrium, the mass balance becomes:

$$
M_{a}(t) R+M_{w f}(t)=M_{a}(t+\Delta t) R^{\prime}
$$

where $R$ is the retardation coefficient $\left(R=1+\rho K_{d} / \theta\right)$ and $R^{\prime}=$ is the three component (soil, porous media, waste form) retardation coefficient.

$$
R^{\prime}=1+\frac{\rho K_{d}}{\theta}+\frac{\rho_{w f} V_{w f} K_{p}}{\theta V_{e}}
$$

The three component model accounts for the differences in mass of the waste form and porous media.

Solving Eqn. 2.16 for the mass in solution at time $\mathrm{t}+\Delta \mathrm{t}$ yields:

$$
M_{a}(t+\Delta t)=\frac{M_{a}(t) R+M_{w f}(t)}{R^{\prime}}
$$

To obtain the mass released over the time interval $\Delta \mathrm{t}$, the difference in the mass contained on the waste form is estimated from:

$$
\Delta M=M_{w f}(t)-\rho_{w f} V_{w f} K_{p} C(t+\Delta t)
$$

The estimate for the concentration can be obtained from Eqns.2.11. To ease the input burden in DUST-MS, it is assumed that the volume of the waste form and the density of the waste form are equal to the finite difference control volume and the bulk density at that location, respectively. In general, this is not the case. However, the partition coefficient always multiplies these two parameters. Therefore, to obtain the appropriate release rate, the partition coefficient should be adjusted to reflect the differences. The adjusted partition coefficient term is: 


$$
\begin{gathered}
K_{p^{\prime}}=K_{p} \frac{\rho_{w f} V_{w f}}{\rho V_{e}} \\
\Delta M=\frac{R\left[M_{w f}(t)-\frac{\rho}{\theta} K_{p^{\prime}} M_{a}(t)\right]}{R^{\prime}}
\end{gathered}
$$

The term $\rho_{\mathrm{wf}} \mathrm{V}_{\mathrm{wf}}$ is the total mass of the waste form. If the mass of the waste form is much less than the mass of the porous media, the partition coefficient should be adjusted downward as given in Eq.. 2.20. Using this assumption and performing a few algebraic manipulations leads to the expression: DUST-MS takes this mass released and immediately adjusts the concentration within the control volume using the following expression:

$$
C_{\text {new }}(t)=C(t)+\frac{\Delta M(t)}{\theta R V_{e}}
$$

The adjusted concentration $\mathrm{C}_{\text {new }}$ represents the equilibrium concentration between the soil, waste form and aqueous solution. Typically, the adjustment is fairly substantial at the first time step after container failure. This is because the waste form and solution are not initially at equilibrium. After the initial adjustment, subsequent adjustments are generally much smaller as equilibrium is maintained by direct incorporation of a term into the transport equation to model the partitioning between the waste form and solution.

Examining Eq..2.21 the following limiting behavior can be determined. In the case of no partitioning $\left(\mathrm{K}_{\mathrm{p}}==0\right)$, the entire inventory is released over the first time step. In the other extreme, as the partition coefficient becomes large, the amount sorbed can become negative. That is the waste form will act as a sink to absorb material. Another interesting point about the release equation is that it accounts for sorption on the soil. For example, if the modified partition and distribution coefficients are equal and much greater than unity, then $\mathrm{R}$ ' is approximately twice the value for $\mathrm{R}$ and the mass released at the first time step $(\mathrm{C}(0)=0)$ is about $1 / 2$ of the total inventory. If the homogenization process has one part waste per ten parts of porous media by mass $\left(K_{p}==1 / 10 K_{p}\right)$ larger releases will occur than if the reverse was true. This reflects the amount of mass available for sorption in the porous media and waste form. Equation XX also demonstrates that if the system starts at equilibrium, the release is zero.

During the time step, equilibrium is maintained by placing the equilibrium relationship directly into the transport equation and obtaining the modified retardation coefficient, R'. This approach has the advantage of being an implicit approach and is incorporated into the DUST-MS computer code. The fact that the release can be recast into a form that modifies the transport equation by R' is an important consideration. It demonstrates that using a partition coefficient and a 
distribution coefficient can be viewed as changing the retardation coefficient in the transport equation. The analyst must insure that sorption is not excessively represented. This can be done by appropriate choice of the retardation parameters $\left(\mathrm{K}_{\mathrm{d}}\right.$ and $\left.\mathrm{K}_{\mathrm{p}}\right)$.

Discussion of the treatment of modifying the transport equation to account for waste form partitioning is presented in section 2.2

\subsubsection{Diffusion Release}

This conceptual model for release assumes that the wastes are uniformly and homogeneously distributed throughout a solidified waste form and that diffusion is the only important process. Under these conditions, analytical solutions for the release rate from the waste forms can be obtained for a variety of geometries. However, exact methods for treating ingrowth of progeny are unavailable. DUST-MS has an approximate method for the analytical models that is exact if the parnt and prgeny have identical wasteform diffusion coefficients. However, DUST-MS also provides a finite-difference wasteform release model that more accurately models ingrowth. When the parent and progeny have different wasteform diffusion coefficients, it is recommended to use the finite difference waste form release models, section 2.5.5.

The DUST-MS code permits selection form three different analytical diffusion release rate models:
a) rectangular geometry
b) cylindrical geometry,
c) spherical geometry.

All of these models assume that the concentration at the boundary is zero. The rectangular model simulates a three-dimensional waste form with unique dimensions in each direction. The cylindrical model simulates a cylindrical waste form of fixed height and radius. The spherical model simulates release from a homogeneous sphere.

. All three analytical models solve the diffusion equation with radioactive decay,

$$
\frac{\partial C_{i}(x, t)}{\partial t}=D_{i} \nabla^{2} C_{i}(x, t)-\lambda_{i} C_{i}(x, t)
$$

where $\mathrm{C}(\mathrm{x}, \mathrm{t})\left(\mathrm{g} / \mathrm{cm}^{3}\right)$ is the concentration within the waste form, $\mathrm{D}_{\mathrm{wf}}\left(\mathrm{cm}^{2} / \mathrm{s}\right)$ is the waste form diffusion coefficient, $\lambda\left(\mathrm{s}^{-1}\right)$ is the radioactive decay constant, $\mathrm{x}$ is the spatial location vector, and $\mathrm{t}$ is the time since container failure. The diffusion time does not start until the container fails. All references to time in this section refer to the time since the diffusion process starts. The DUST-MS code allows selection of a unique diffusion coefficient for each radionuclide.

The initial condition for all three models is: 


$$
C_{i}(x, 0)=C_{i, 0}
$$

$\mathrm{C}_{\mathrm{i}, 0}$ is the initial concentration in the pore waters of the waste form and $\mathrm{x}$ represents the position vector, $(\mathrm{x}, \mathrm{y}, \mathrm{z}$ for rectangular coordinates, $\mathrm{r}, \mathrm{z}$ for cylindrical coordinates, and $\mathrm{r}$ for spherical coordinates). It is estimated from the initial inventory and waste form volume.

\subsubsection{Rectangular Waste Form}

The first analytical model simulates a rectangular waste form with dimensions $2 \mathrm{a}, 2 \mathrm{~b}$, and $2 \mathrm{c}$ along the $\mathrm{x}, \mathrm{y}$, and $\mathrm{z}$ directions respectively.. The boundary conditions are zero flux through the mid-planes of the waste form $(x-z=0 ; z-y=0$, and $x-y=0)$. For example, at the $x-y=0$ plane, and zero concentration at the boundary.

$$
\begin{aligned}
& \frac{\partial C_{i}(0,0, z, t)}{\partial z}=0 \\
& C_{i}\left(x_{b}, y_{b}, z_{b}, t\right)=0
\end{aligned}
$$

Where $\mathrm{x}_{\mathrm{b}}, \mathrm{y}_{\mathrm{b}}, \mathrm{z}_{\mathrm{b}}$ denotes any point on the boundary of the waste form.

The concentration at any point in the waste form is given by:

$$
C(x, y, z, t)=\frac{64}{\pi^{3}} C_{i, 0} e^{-\lambda t} T_{x}(a, t) T_{y}(b, t) T_{z}(c, t)
$$

where $\lambda$ is the radioactive decay constant and all other variables have been defined. The function $\mathrm{T}$ written in terms of the generic dimension variable $u$ is :

$$
T_{u}(L, t)=\sum_{n=1}^{\infty} \frac{(-1)^{n+1}}{(2 n-1)} e^{-\left[\frac{(2 n-1) \pi}{2 L}\right]^{2} D t} \cos \left[\frac{(2 n-1) \pi u}{2 L}\right]
$$

To obtain the total release rate across each of the six faces of the parallelogram, equation 18 is differentiated and multiplied to get the flux out of the surface. The flux is integrated over the surface area to yield the release rate, $Q_{d}$, across the face $\mathrm{x}=\mathrm{a}$ to give: where: 


$$
\begin{gathered}
Q_{d, x=a}(\lambda, t)=\frac{512}{\pi^{4}} C_{i, o} D_{w f, i} \frac{b c}{a} e^{-\lambda t} S_{q}(b, t) S_{q}(c, t) S_{d}(a, t) \\
S_{q}(b, t)=\sum_{n=1}^{\infty} \frac{e^{-\left[\frac{(2 n-1) \pi}{2 b}\right]^{2} D t}}{[2 n-1]^{2}} \\
S_{d}(a, t)=\sum_{n=1}^{\infty} e^{-\left[\frac{(2 n-1) \pi}{2 a}\right]^{2} D t}
\end{gathered}
$$

and

Analogous expressions are derived for each face of the waste form and the total release is estimated through summation of the release from the different faces of the parallelogram.

Techniques to speed the convergence of the series in Eq. 2.31 and 2.32 have been developed \{Pescatore, 1991] and are discussed in the cylindrical waste form release model section which follows.

\subsubsection{Cylindrical waste form}

The second analytical model simulates a cylinder with radius, $\mathrm{R}$, and heigth, $\mathrm{H}$. At the edge of the waste form, the contaminant concentration is zero. As with the finite plane model, this

$$
\begin{gathered}
C(R, z, t)=C(r, 0, t)=C(r, H, t)=0 \\
\frac{\partial C(0, z, t)}{\partial r}=0
\end{gathered}
$$

boundary condition causes the maximum concentration gradient to be maintained and leads to the highest release as compared to other possible boundary conditions. The boundary conditions are:

The solution for the cumulative fractional release, CFR, is the product of two infinite series [Nestor, 1980]:

$$
C F R=1-\frac{32 S_{q} S_{d}}{\pi^{2}}
$$


where

$$
\begin{gathered}
S_{c}=\sum_{m=1}^{m=\infty} \frac{1}{\beta_{m}^{2}} e^{\frac{-\beta_{m}^{2} D_{w f} t}{R^{2}}} \\
S_{q}=\sum_{n=1}^{n=\infty} \frac{1}{(2 n-1)^{2}} e^{\frac{-(2 n-1)^{2} \pi^{2} D_{w f} t}{H^{2}}}
\end{gathered}
$$

where the $\beta_{\mathrm{m}}$ are the zeroes of the zero-th order Bessel function.

The American Nuclear Society has published a standard. ANS 16.1, which describes procedures for testing and analyzing the release form solidified low-level radioactive wastes [ANS, 1986]. In ANS 16.1, Eq.. 2.37 is the recommended approach for estimating releases from cylindrical waste forms provided the release is greater than $20 \%$. When the release is less than $20 \%$ ANS 16.1 recommends using the predicted release based on treating the waste form as a semi-infinite medium. The reason for this is the poor convergence of the semi-infinite series at low CFR values and the minor difference in predicted CFR between the semi-infinite medium model and the finite cylinder model, Eq.. 2.37 when the CFR is less than 0.2 Even though the difference is small, the semiinfinite model always predicts greater release than the series solution. Thus, at the time step when

$$
C F R=\frac{2 S A_{w f}}{V_{w f}} \sqrt{\frac{D_{w f} t}{\pi}}
$$

the switch is made, an under prediction of release at that time step will occur.

The semi-infinite waste form model for release is:

where $\mathrm{Sa}_{\mathrm{wf}}$ is the surface area of the waste form and $\mathrm{V}_{\mathrm{wf}}$ is the volume of the waste form.

An improved method for calculating the series solution has been developed [Pescatore, 1991] which permits truncation of the series after only a few terms. In this approximation

$$
C F R=1-\frac{32 S_{q, N} S_{c, M}}{\pi^{2}}
$$

where 


$$
S_{q, N}=\sum_{n=1}^{n=N-1} \frac{1}{(2 n-1)^{2}} e^{\frac{-(2 n-1)^{2} \pi^{2} D_{w f} t}{H^{2}}}
$$

and

$$
\begin{gathered}
-\frac{1}{R f_{M}} \sqrt{\pi D_{w f} t} \operatorname{erfc}\left[\beta_{M} \frac{\sqrt{D_{w f} t}}{R}\right] \\
+\frac{N}{(2 N-1)^{2}} e^{\frac{-(2 N-1)^{2} \pi^{2} D_{w f} t}{H^{2}}} \\
-\frac{\sqrt{\pi D_{w f} t}}{2 H} \operatorname{erfc}\left[(2 N-1) \pi \frac{\sqrt{D_{w f} t}}{H}\right] \\
f_{M}=\pi-\frac{S_{c, M}=\sum_{m=1}^{m=M-1} \frac{1}{\beta_{m}^{2}} e^{\frac{-\beta_{m}^{2} D_{w f} t}{R^{2}}}}{8 \pi \frac{\sqrt{\pi} D_{w f} t}{M^{2}} 2 H} \operatorname{erfc}\left[(2 N-1) \pi \frac{\sqrt{D_{w f} t}}{H}\right]
\end{gathered}
$$

Through testing it has been determined that setting $\mathrm{N}$ and $\mathrm{M}$ to ten provides sufficient accuracy.

DUST-MS uses both the semi-infinite model. Eq.. 2.40 and the series solution, Eq., 2.41 to estimate releases. Estimated releases from both equations are compared, DUST-MS selects the minimum of these two estimates. At early times the series solution over predicts release as it is not fully converged. At later times, the semi-infinite media model predicts higher releases as depletion in the waste form is not simulated. Similar remarks apply to the analytical models for release from rectangular and spherical waste forms.

The equations 2.37 and 2.38 estimate the cumulative fractional release, not the release rate, 
the quantity needed in the transport equation. The release rate per unit volume due to diffusion over the time step is estimated from the following expression:

$$
Q_{d}=\frac{C F R(t+\Delta t)-C F R(t)}{V_{e}} M_{o} e^{-\lambda t}
$$

where $\mathrm{M}_{\mathrm{o}}$ is the original mass in the waste form.

\subsubsection{Spherical Waste Form}

Release from a homogeneous spherical waste form of radius $r$ which has a uniform initial concentration is estimated from the following expression [Pescatore, 1991] for cumulative fractional

$$
C F R=1-\frac{6}{\pi^{2}} S_{s}(t)
$$

release:

$$
S_{s}=\sum_{n=1}^{n=\infty} \frac{e^{\frac{-n^{2} \pi^{2} D_{w f} t}{R^{2}}}}{n^{2}}
$$

where the series $S_{s}(t)$ is:

To close the series, the following approximation is made:

$$
S_{s, N}=\sum_{n=1}^{n=N-1} \frac{e^{\frac{-n^{2} \pi^{2} D_{w f} t}{R^{2}}}}{n^{2}}+\frac{2 N+1}{2 N^{2}} e^{\frac{-N^{2} \pi^{2} D_{w f} t}{R^{2}}}+\frac{\sqrt{\pi^{3} D_{w f} t}}{R} \operatorname{erfc}\left[N \pi \frac{\sqrt{D_{w f} t}}{R}\right]
$$

Analogous to the cylindrical case, the cumulative fractional release is calculated and Eq.. 2.48 is used to estimate the release rate over the time step.

2.5.2.4 Approximate treatment of ingrowth in the analytical diffusion models 
The analytical diffusion release models described in section 2.5 are all based on the assumption that the production of the radionuclide due to radioactive decay of other species does not occur. When ingrowth occurs, the approximation is made that the distribution of mass due to ingrowth is identical to the distribution of mass in the waste form generated by diffusion. This is equivalent to assuming that the parent and progeny have identical diffusion coefficients.

This approximation is not true in general due to the potential for differing diffusion coefficients of the parent and progeny. However, the approximation does conserve mass. If the parent moves at a slower rate than the progeny, this approximation will under predict release. The cause for this behavior is the parent's diffusion profile will be shifted more towards the center of the waste form as compared to the faster moving progeny. In the limit that the parent is immobile, the production of progeny will occur uniformly throughout the waste form. Therefore, more progeny will be produced closer to the edge of the waste form than would be predicted by the model.

In general, if ingrowth of progeny occurs it is strongly recommended to not use the analytical diffusion models. Instead the finite-difference diffusion models described in section 2.5 .5 should be used.

\subsubsection{Uniform Degradation Release}

The conceptual model for uniform release assumes that the waste form releases the radioactivity at a uniform rate. Typically this release behavior would be caused by degradation reactions occurring at the surface of the waste form (i.e., metal corrosion, glass dissolution, etc.). Due to the wide variety of waste forms/waste streams that this model may be used to represent and to avoid confusion with the term dissolution which has a specific definition, the name has been generalized to the uniform degradation release model.

In DUST-MS each radionuclide may be assigned a unique uniform release rate. In the

$$
Q_{u}=\frac{u_{i} M_{0}}{V_{c v}} e^{-\lambda t}
$$

absence of ingrowth the release rate per unit volume is estimated from:

where $u_{i}$ is the fractional release rate, and other quantities have been previously defined. If ingrowth occurs, the mass available for release, $\left(\mathrm{M}_{\mathrm{o}} \mathrm{e}^{-\lambda t}\right)$ is generalized to the mass available at that time $M(t)$ based on ingrowth as well as decay. This is discussed further in section 2.3.7

\subsubsection{Total Waste-Form Release Rate}

DUST-MS permits each waste form to release mass by any of the release mechanisms 
described in section 2.3.1 (rinse with partitioning), 2.3.2 (diffusion), and 2.3.3 (uniform). In the absence of solubility constraints, the release rate per unit volume to the transport equation is the sum of the three individual release rates. If solubility limits release the source term to the transport equation is reduced to a level such that the solution concentration equals the solubility limit. This procedure is discussed in more detail in section 2.3.6.

\subsubsection{Influence of Localized Failure on Release}

If there is localized failure, the intact portion of the container still provides a barrier to release from the waste form-container system. The conceptual model for release from partially failed containers assumes that once a container is breached, there will be a steady flow of water into and out of the container. The flow rate is estimated from the Darcy velocity multiplied by the fractional area of container that has failed. Other conceptual models could be envisioned. For example, if the breach occurred at the top, water could begin to fill the container and a bathtub could form until the water level reached the lowest breached area. This could potentially lead to a large pulse of radioactive contaminants. However, accurately predicting the location of failures around the container is beyond the state-of-the-art. The selection of the model for instant flow through the container once a breach has occurred was selected to provide an estimate of the earliest time that releases could occur.

An important aspect of the strategy used to represent localized failure is the treatment of waste form releases. The diffusion model assumes that once the waste form is contacted by water, the release process begins and is given by the analytical models, section 2.3. These models are unaffected by the breached area. Similarly, release from the uniform release model is also calculated independent of the breached ratio. To adjust for the fact that the entire container is not breached, the release rate per unit volume from the diffusion and uniform release models is reduced by the breach ratio, (area of the breach over the total container area). Mass not released is transferred to the rinse release model. This permits the analytical solutions to be retained while still taking credit for partial container failure. Release from the rinse model is treated as release from a mixing cell, all of the mass available for rinse release (including mass transferred from the diffusion and uniform release models) is assumed to enter into a mixing cell. The release from the mixing cell is the product of the Darcy velocity, breached ratio, the area available for flow, and the concentration in the mixing cell.

A number of assumptions have been made in developing the waste form release models in the case of partial container failure. Data to support these assumptions (or any other possible set of assumptions) does not exist due to the long time frame over which these processes will occur and the difficulty of experimentally measuring the entire process. Due to the speculative nature of the assumptions the model results are also speculative and care should be used when interpreting the results.

\subsubsection{Finite Difference Waste-Form Release Model}

DUST-MS provides an alternative approach to simulating release from the waste form as 
compared to the analytical models presented in section 2.5.3. In this approach all processes, rinse with partitioning, diffusion, and uniform degradation are modeled simultaneously using the method of finite differences. This numerical approach offers the following advantages over the analytical models:

- simultaneous treatment of all release processes,

- direct coupling of diffusion release to the concentration of the contacting solution, and

- method for modeling ingrowth of radionuclides in the waste form which are released by the diffusion process.

The limitations of the numerical models as compared to the analytical models is the possibility for numerical error and the increased computational requirements. In addition, the finite difference models assume one-dimensional geometry (planar, cylindrical, or spherical) as compared to the three-dimensional analytical diffusion release models. Experience has shown that calculation of waste form release is a small fraction of the entire DUST-MS calculation. Therefore, the penalty in slower execution time is not large. Testing of the finite difference models and comparison to the analytical models show excellent agreement under most situations.

The finite difference model retains the distinction between mass available for release due to each mechanism (rinse with partitioning, diffusion, and uniform degradation). The finite difference model assumes that the waste form is a porous media with a liquid phase which permits movement of aqueous species and a solid phase which releases species due to dissolution of the surface of the waste form. This dissolution will remove the outer layer of the waste form. This alters the diffusion release in two ways. First, mass in the pore waters of the dissolved region is released. Second, the size of the waste form, and therefore diffusion distance to the boundary, decreases in time. These effects demonstrate the direct coupling between the dissolution and diffusion models which was absent from the analytical models. A discussion of when this coupling is important is provided in [Sullivan, 1991].

$$
\theta \frac{\partial C_{i}}{\partial t}=\nabla \bullet \theta D_{w f, i} \nabla C_{i}+\nabla \bullet V_{w f} C_{i}-\lambda_{i} \theta C_{i}+\sum_{j=l-N}^{j i} f_{i j} \lambda_{j} C_{j}+S_{i}
$$

The general equation for predicting release with all three mechanisms is: where:

$\theta$ is the moisture content within the wasteform. For simplicity this is taken as the moisture content within the finite difference control volume .

$\mathrm{C}_{\mathrm{i}}$ is the concentration of the $\mathrm{i}^{\text {th }}$ radionuclide $\left(\mathrm{g} / \mathrm{cm}^{3}\right)$,

$\mathrm{D}_{\mathrm{wf}, \mathrm{I}}$ is the waste form diffusion coefficient for the $i^{\text {th }}$ radionculide $\left(\mathrm{cm}^{2} / \mathrm{s}\right)$,

$\mathrm{V}_{\mathrm{wf}}$ is the volumetric flow rate per unit area through the waste form $(\mathrm{cm} / \mathrm{s})$,

$\lambda_{\mathrm{i}}$ is the radioactive decay constant for the ith radionuclide $(1 / \mathrm{s})$

$\mathrm{f}_{\mathrm{ij}}$ is the fraction of decays of species $\mathrm{j}$ that form species $\mathrm{I}$, and

$\mathrm{S}_{\mathrm{i}}$ is the source/sink term within the waste form. This could be used to represent coupling between the immobile and mobile phases in the waste form due to precipitation or sorption. 
Eqn. 42 accounts for ingrowth directly. Theoretically there is one equation for each type of mass (rinse, diffusion, and general degradation). However, the rinse release model simulates a surface process and solution of Eqn 42 is not required. The mass released due to rinse with partitioning is treated using the approach described in section 2.3.1.

Although Eqn 42 is quite general, to solve this equation numerically is difficult when the waste form is dissolving. In this case, the volumes represented by the finite difference mesh may change in time. For example, the outermost volume will decrease as dissolution of the surface

$$
y=\frac{x}{L(t)}
$$

occurs. To avoid this problem a change in the position variable is introduced.

Where: $y$ is a dimensionless distance variable

$\mathrm{x}$ is the dimensioned distance variable $(\mathrm{cm})$

$\mathrm{L}(\mathrm{t})$ is the length (radius) of the waste form at time $\mathrm{t}$.

In the transformed variable space, the waste form lies between the region of 0 and 1 at all times . However, the distance variable y is now a function of time. Differentiating Eqn. 43 with respect to time gives:

$$
\frac{d y}{d t}=-\frac{y}{L} \frac{d L}{d t}
$$

The time derivative of concentration in the transformed plane becomes:

$$
\frac{\partial C(y(t), t)}{\partial t}=\frac{\partial C}{\partial t}+\frac{\partial y}{\partial t} \frac{\partial C}{\partial y}
$$

The rate of change in waste form length with time is the dissolution velocity, $d L / d t=u(t)$. Using this gives:

$$
\frac{\partial C_{i}(y, t)}{\partial t}=\frac{\partial C_{i}}{\partial t}-\frac{u_{i} y}{L(t)} \frac{\partial C}{\partial t}
$$


The dissolution velocity is negative since the length of the waste form decreases in time. For consistency with the uniform degradation model, the dissolution velocity is assumed to be solubility limited and is taken to be:

$$
u_{i}(t)=-u_{i, o} L_{o}
$$

where: $\quad \mathrm{u}_{\mathrm{o}}$ is the fractional degradation rate, section $2.3 .3(1 / \mathrm{s})$,

$\mathrm{L}_{\mathrm{o}}$ is the original waste form half width (radius) $(\mathrm{cm})$,

Using this definition, different radionuclides may have different dissolution rates due to the choice of fractional degradation rate or solubility limit.

Using the transformation in position and the definition of dissolution velocity the mass balance equation for the waste form becomes:

$$
\begin{gathered}
\theta \frac{\partial C_{i}}{\partial t}=\frac{\theta D_{w f, i}}{L(t)^{2}} \frac{\partial^{2} C_{i}}{\partial y^{2}}+\frac{V_{w f}}{L(t)} \frac{\partial C_{i}}{\partial y}+\frac{\theta u_{i}(t) y}{L(t)} \frac{\partial C_{i}}{\partial y} \\
+N_{g}\left[\frac{-\theta D_{w f}}{y L(t)^{2}} \frac{\partial C_{i}}{\partial y}+\frac{V_{w f} C_{i}}{y L(t)}\right]-\lambda_{i} \theta C_{i}+\sum_{j=l-N}^{j_{i} i} f_{i j} \lambda_{j} C_{j}+S_{i}
\end{gathered}
$$

where $\mathrm{N}_{\mathrm{g}}$ takes the value of 0 for plane geometry, 1 for cylindrical geometry, and 2 for spherical geometry. Eqn. 2.53 forms the basis for the finite difference equations used to solve for the concentration related to diffusive species and immobile dissolving species. In Eqn. 2.53 the parameter $\theta$ is the volumetric content of the phase being modeled. For the mobile phase, it is the moisture content. For the immobile phase, it is the fraction of the waste form in the solid phase, (1 $\eta$ ), where $\eta$ is the porosity of the waste form. In DUST-MS, the velocity of flow through the waste form, $\mathrm{V}_{\mathrm{wf}}$ is set to zero. For the immobile phase, Eqn. 2.53 greatly simplifies to the following:

$$
(1-\eta) \frac{\partial C_{i}}{\partial t}=\frac{(1-\eta) u_{i}(t) y}{L(t)} \frac{\partial C_{i}}{\partial y}-\lambda_{i} \theta C_{i}+\Sigma_{j=1-N}^{j_{i} i} f_{i j} \lambda_{j} C_{j}+S_{i}
$$

where $C_{i}$ is the concentration of the immobile species in the waste form. Eqn 2.54 is used to calculate the immobile species concentrations in the waste form in DUST-MS. If the initial condition is a uniform concentration and the production due to sources and radioactive decay are also uniform, Eq.. 2.54 predicts a uniform concentration of immobile species in the waste form $(\mathrm{dC} / \mathrm{dy}=0$ in the waste form). This is identical to the assumptions used to generate the analytical 
model. To improve the computational efficiency of DUST-MS, solution of Eq. 2.54 could be omitted and the releases due to degradation could be accounted modeled using the analytical models.

The initial conditions used in DUST-MS is that initially the mass is homogeneously distributed within the waste form. This prevents the need to input detailed information on the distribution of contaminant within the waste form. The only information that is required is the waste form inventory and volume. Conceptually, non-uniform initial conditions could be handled by the finite difference approach provided this information was provided.

The boundary condition used to calculate diffusive release from the waste form is the solution concentration in the finite difference control volume calculated by the transport equation. This allows direct coupling of the aqueous concentration to waste form release. This coupling is explicit as the aqueous concentration at the beginning of the time step is used and releases do not change this concentration instantly. That is, release is calculated from the finite difference model and the mass release rate per unit volume of the control volume is provided to the transport calculation. The aqueous concentration is calculated as a result of the source as well as transport processes.

Since the coupling between the aqueous solution concentration and the waste form concentration is explicit, numerical problems can arise. The potential for numerical problems is further exacerbated by a potential inconsistency between the waste form and the solution volumes. DUST-MS permits the user to supply a waste form volume and a volume for the disposal facility (area of the facility multiplied by the finite difference cell size). Problems will arise if the waste form volume is selected to exceed the facility volume. Although this is non-physical, it is permitted by DUST-MS. For example, one method to compare the 1-D finite difference model to the 3-D analytical models is to set the dimensions to large values such that the simulation is almost 1-D. As an example, to simulate radial release from a $25 \mathrm{~cm}$ radius waste form using the analytical model, the volume of the waste form could be set to $10^{10}$.. This large volume would minimize releases through the end of the cylinder as calculated by the analytical model. Now, however, if the volume of the facility is much smaller than the volume of the waste form, the aqueous solution concentration will exceed that in the waste form. In principle, mass would then diffuse back into the waste form. This can lead to numerical instabilities. To avoid this problem when using the finite difference release model, if the solution concentration exceeds the concentration in the waste form, diffusive release into the wastes is not permitted. In general, the volume of the waste form should be much less than the volume of the facility and this should be accounted for through proper choice of input.

The treatment of solubility limited release is provided in section 2.3.6.

2.5.5.1 Qualitative comparison of predicted releases based on the finite difference and analytical release models

The rinse models in the finite difference and analytical models are identical and will give identical release results if solubility limits do not apply. 
The diffusion models in the finite difference and analytical models should also give essentially identical predicted release results if the following conditions apply.

- Dissolution does not substantially decrease the size of the waste form during the time required for diffusion release.

- Solubility does not limit release

- Ingrowth of progeny does not occur.

If substantial dissolution of the waste form does occur, the finite difference model will predict higher releases due to the shorter effective diffusion length as well as dissolution of the wastes.

The general degradation models in the finite difference and analytical procedures are slightly different conceptually. The analytical model assumes a fractional release rate independent of geometry. In the finite-difference models the conceptual model assumes a surface process which dissolves the waste form. The total mass release rate over a time step is proportional to the waste form surface area. For a plane waste form, this is constant and the results of the finite-difference model should be identical to the analytical model, section 2.3.3. However, for spherical or cylindrical waste forms, as the waste form radius decreases due to degradation processes, the surface area also decreases. At early times, the finite difference model for degradation of a spherical or cylindrical waste forms predicts higher releases than either the plane finite difference model or the analytical model. This is because the waste form volume dissolved per unit time in a curved waste form is initially greater than for a plane form. Verification test results for this process and analytical expressions for the releases from cylindrical waste forms for radioactive contaminants that do not experience ingrowth are presented in the BLT-MS input guide as well as section 5 in this report.

Ingrowth is modeled in both the analytical and finite-difference degradation models. Since the contaminants available for degradation release are assumed to be immobile within the waste form, their distribution remains uniform at all times. Therefore, the analytical and plane finite difference models should give similar results when ingrowth occurs. The cylindrical and spherical geometry degradation models results will differ due to the geometric effect discussed in the previous paragraph.

\subsubsection{Solubility Limited Release}

In DUST-MS solubility limited release is treated through attempting to limit the mass release rate per unit volume to the transport equation to a level such that the solubility limit of the radionuclide is not exceeded. DUST-MS uses an implicit procedure to estimate the amount of mass that can be released without exceeding solubility limits. This approach calculates the source using the waste for release models without adjusting for solubility limits. The solution concentrations are then calculated and compared to the solubility limit. If the solubility limit is not exceeded in any of the waste form source nodes, the calculation proceeds to the next time step.

If the solubility limit is exceeded, at each location where the predicted concentration exceeds the limit, the matrix equations are adjusted to force the solution concentration to the 
solubility limit. The new set of matrix equations are solved and the waste form release rates for each release mechanism are calculated in a consistent fashion with the release required to reach the solubility concentration. The waste form release rates are then used to adjust the mass available for release at subsequent times in the calculation. When the solubility limit is exceeded, mass predicted to be released due to each mechanism is reduced by the same fraction. . For example, if the models predicted 10\% release from diffusion, 30\% from degradation processes, and $60 \%$ from rinse processes and due to solubility constraints only 2 units of mass was released during the time step, the models would release 0.2 units by diffusion ( $10 \%$ of the total), 0.6 units by degradation $(30 \%)$, and 1.2 units by rinse $(60 \%)$.

For the diffusion model, if solubility limited release occurs, the conceptual model for release takes all of the mass predicted to diffuse out of the waste form and assume that any mass predicted to be released by diffusion that would cause the solution concentration to exceed the solubility limit precipitates on the surface. During subsequent time steps this 'precipitated' mass is available for release due to the rinse mechanism once the solution concentration drops beneath the solubility limit. This precipitation model is also used for the dissolution mass that is not released due to solubility constraints. This approach insures that the cumulative release does not exceed the predicted cumulative reelase in the absence of solubility limits.

\subsubsection{Treatment of Chain Decay in the Waste-Form}

Decay of radionuclides to produce other radionuclides can be an important process for uranium and thorium containing wastes. Progeny of these nuclides may be more mobile and provide higher doses per unit intake. DUST-MS accounts for production of progeny in the waste form prior to release and during transport. Definition of the decay chains is controlled by user supplied input. DUST-MS permits multiple decay chains and branching of decay chains. Guidance on how to simulate these processes within the DUST-MS framework is provided in section 6 and 7.

The treatment of chain decay is complicated by the multiple release models permitted in DUST-MS. Within a single waste form each radionuclide can have unique release mechanisms. For example, consider a three member decay chain. Species 1 could have all of the mass designated to be released via diffusion. While for species 2 it could be assumed that mass release is a uniform degradation process and species 3 could be assumed to release via rinse with partitioning. To complicate matters, each species may designate a fraction of the initial inventory to each of the release mechanisms. There is no obvious method for determining the release mechanism for the decay product, i.e., should the release mechanism remain that of the parent or of the progeny. This is somewhat of an artificial result of defining multiple release mechanisms for a waste form. However, cases could be envisioned where the parent is released via diffusion while the progeny could be incorporated into the solid phase of the waste form and released by dissolution. Generally, data on the release from a waste form of different radionuclides in a decay chain does not exist. For this reason, it is strongly recommended that when using DUST-MS to simulate decay chains that all members of the chain have the same fraction of mass available for release to each mechanism. Unusual results may occur when altering the release mechanisms on a radionuclide specific basis 
for members of a decay chain.

In DUST-MS the following assumptions are used when different release mechanisms are specified for the members of a decay chain:

- Prior to container breach, the mass that decays to the progeny are apportioned consistent with the input choices of release mechanism. For example, if the release mechanisms assigned to species 1 are $30 \%$ diffusion release and $70 \%$ rinse release while for species $2100 \%$ uniform degradation release is specified, all of the mass that decays to species 2 will be assigned for release via the degradation model.

- After container breach, the release mechanism for the decayed mass remains the same as the parent. Using the above example after container failure, all of the mass that decays from the diffusion release portion is assigned to diffusion release for species 2 and similarly the decayed rinse mass is assigned to be released via the rinse mechanism for species 2 . None of the decayed mass is assigned to the degradation release mechanism. This approximation was selected to avoid complications when solubility limited release occurs and to simplify the treatment of the finite difference equations. In the finite difference approach, with this assumption, mass that decays is transferred directly into the next member of the chains finite difference equation. Transferring mass among release mechanisms is possible, but difficult to achieve numerically in the finite difference equations.

The actual process for estimating ingrowth is accomplished by using an explicit estimate of decay at the beginning of the time step. Prior to breach and at all times for the analytical models, the

$$
\Delta M_{j}=\sum_{i=1-N}^{i j j} f_{i j} M_{i}(t)\left[1-e^{-\lambda_{i} \Delta t}\right] \frac{\operatorname{ATM}(i)}{\operatorname{ATM}(j)}
$$

decay mass produced over a time step, $\Delta t$, for the $\mathrm{j}^{\text {th }}$ member of a chain is estimated from: Where $f_{i j}$ is the fraction of decays of species I that produce species $j, M_{i}(t)$ is the mass available at time $t$, and ATM is the atomic mass. This ratio of atomic masses correction term is needed because the mass of the parents and progeny per atom are not identical. Eq.. 2.3.47 is repeated for each release mechanism and the mass is apportioned to the release mechanisms of the progeny according to the procedure described above.

After breach, for the waste form finite difference release model, the decay is treated directly in the model equations as defined in the summation expression found Eqns.48 and 50.

The coupling between different species due to radioactive decay is treated explicitly. That is, the mass or concentration value used to estimate ingrowth is that at the beginning of the time step. This approach removes the need to simultaneously solve each transport equation to determine the appropriate values to be used for ingrowth..

2.5.8 Selection of Waste Form Release Parameters 
Due to the wide range of waste forms used in low-level waste disposal there is extremely little information on releases that is waste form specific. Even when there is information, it tends to focus on the short-lived radionuclides that have the highest activity in the wastes but not the highest impact in terms of potential dose to the public as evaluated through performance assessments. Typically, long-lived, mobile radionuclides are responsible for the majority of the estimated dose.

In estimating waste form release parameters it is recommended to use the rinse model without partitioning when contaminant specific, waste form specific data are unavailable. This model provides the highest release rate (instantaneous release of the entire inventory at the time of container failure) and therefore, highest doses through the ground water pathway in most instances (if ingrowth is of concern, the high release rate may cause releases from the facility to occur prior to substantial ingrowth and this may under predict release of progeny).

For many solidified waste forms it can be shown that diffusion is the rate controlling release process. If this is the case, and if waste-form specific contaminant specific data are unavailable, modeling of diffusion release can be accomplished with a waste form diffusion coefficient of $10^{-6}$ $\mathrm{cm}^{2} / \mathrm{s}$. This value is the maximum acceptable value for a waste form diffusion coefficient [Lohaus, 1991]. A compilation of diffusion coefficients for solidified waste forms is presented in [Sullivan, 1989].

For metallic waste forms (activated metals) and glass waste forms surface degradation processes often control release. Estimates for metallic corrosion rates can be obtained by examining the soil corrosion data [Romanoff, 1957; Gerhold, 1981]. A discussion of this data and its applicability to modeling waste form release is in [Sullivan, 1989]. Similar, glass dissolution rates may be estimated from values reported in the literature. Often, due to the uncertainties in these processes, waste form release rates due to degradation processes are estimated based on values at the high end of those reported in the literature.

In all cases when there are large uncertainties in the release rate parameters, it is recommended that multiple analyses be performed sampling from the range of expected release rate parameters.

The waste form properties are independent of the transport properties and care must be taken to insure that properties are not accounted for twice. For example, if DUST-MS is used to model contaminated soils, it is inappropriate to use partitioning with waste form release and a distribution coefficient in the soil. If the soil is in a container, the rinse model should be used. After release, the contaminant will sorb to the soil based on the distribution coefficient used in the transport section of the code. In general, extreme care must be used when selecting to use a non-zero value for the waste form partitioning coefficient. Negative releases (i.e., sorption onto the waste form) can occur and the potential for accounting for sorption twice (in waste form release and during transport) is large. The waste form partitioning model has been the most widely misused of all DUST and BLT release models. 
The partially-failed container will be treated as a mixing cell in which radionuclides released from the wasteform are uniformly mixed within the container. The release rate from the container will be the product of the container flow rate and the mixing cell concentration as calculated based on the various release mechanisms. This is identical to the approach used in the BLT computer code [Sullivan, 1989].

\subsubsection{Wasteform Release with Distributed Container Failure}

DUSTMS-D is a one-dimensional (1-D) model that predicts the release and transport of contaminants disposed in the subsurface. The conceptual model collapses the 3-D physical system down to 1-D mathematical representation. This implies that there are frequently multiple containers represented in one computational cell by a single effective container. This effective container can fail at a specified time that represents the mean time to failure of all containers represented in the computational volume. However, in practice it is probable that the containers will fail over a distribution of times. To account for this, the single failure time is generalized to a distribution of failure times. In theory, the distribution can be any function. In most cases, the distribution of failure time approximates known statistical distribution functions such as the uniform, normal, lognormal, or exponential distribution functions.

In general, the total release rate from a set of containers would be the summation of the release rate from each container. Accounting for each container is not computationally feasible. Therefore, in this model, the release rate from all of the wasteforms represented in a single control volume is simulated by a representative release rate from a single wasteform. That is all of the wasteforms within the control volume are lumped into a single effective wasteform with the total inventory for all wasteforms and one set of release rate parameters. Thus, the total release rate is a function of the release rate for the representative waste form and the container failure parameters.

Two situations can be envisioned for describing the release under conditions of distributed container failure. In the first, the waste form release rate is completely independent of the time to failure or distribution of failure times. In this case, the total release rate from all containers is defined as:

Release Rate $=$ Release rate from the wasteform $*$ fraction of failed containers.

An example of this type of behavior is a uniform release for all times. An important example for this type of release would be an exponentially decaying release rate, with the decrease due to radioactive decay.

The second situation occurs when the release rate is dependent on the time of failure. This will be the typical case in waste disposal. In this case, the release rate can be represented as a convolution integral that takes into account the interrelationship between wasteform release and container failure time. The mathematical form is described in detail in the next section. 


\subsubsection{Mathematical Model}

With a distribution of failure times, calculation of release from the waste form becomes more complicated than for a single failure time. To calculate release with a distribution of failures requires the combination of the fraction of containers failed at a given time and the release rate over the time since container failure. This can be represented as a sum over all containers

$$
R(t)=\sum_{j=1}^{n} r_{j}\left(t-\tau_{j}\right) f\left(\tau_{j}\right)
$$

where $\mathrm{R}(\mathrm{t})$ is the total release rate from all waste packages

$r_{j}\left(t-\tau_{j}\right)$ is the release rate from waste package $j$ at time $t-\tau_{j}, t>\tau_{j}$

$\tau_{j}$ is the failure time of the $\mathrm{j}^{\text {th }}$ container, and

$\mathrm{f}\left(\mathrm{t}_{\mathrm{j}}\right)$ is the fraction of containers that fail at time $\mathrm{t}_{\mathrm{j}}$ (in statisitcs this is known as the probability density function)

As an example, consider three containers with three different failure times, (10, 20, and 30 years). The total release rate from all three after 15 years would be:

$R(15)=1 / 3 r_{1}\left(t-\tau_{1}\right)=r_{1}(5) / 3$

Containers 2 and 3 have not failed at this time. Note this approach assumes that the mass is distributed uniformly between the three containers. Therefore, the total release is scaled by the factor of $1 / 3$ which represents the fraction of containers that fail at a given failure time. After 40 years, the release would be:

$$
R(40)=1 / 3 r_{1}(30)+1 / 3 r_{2}(20)+1 / 3 r_{3}(10)
$$

The above approach is appropriate when modeling only a few containers with known failure times. However, when attempting to model a large number of containers it becomes more computationally efficient to represent the failure times with a continuous distribution that represents the range of possible failure times. With a continuous distribution of failure times, Eqn (1) can be generalized as a convolution integral:

$$
R(t)=\int_{0}^{t} r(\tau) \cdot f(t-\tau) \partial \tau
$$

In equation 2 we have assumed that all containers represented by the distribution of failure times have identical release rates. That is, one set of release rate parameters describe all of the containers in this computational volume. This is less general than the example given for the discrete case where each container was allowed to have unique release properties. However, it is required due to the impracticallity of defining a unique set of release parameters that varies as a function of failure time. 


\subsubsection{Special Cases of Probability Density Functions}

c) Instantaneous failure of all containers at time $t_{j}$

The failure distribution function is:

$$
f\left(t-t_{j}\right)=\delta\left(t-t_{j}\right)
$$

where $\delta\left(t-t_{j}\right)$ is the Kronicker delta function.

Use of this function, Eqn (2.63), in Eqn (2.62) gives the release rate as:

$$
\begin{array}{lll}
R(t)=0 & t<t_{j} & \\
R(t)=r\left(t-t_{j}\right) & t>t_{j}
\end{array}
$$

This is the release rate for a single failure time for all containers.

d) Uniform Container Failure Rate

The containers fail at a uniform rate from the beginning time of failure, $t_{b}$, to the ending time of failures, $t_{\mathrm{e}}$. The distribution function is:

$$
\begin{aligned}
f\left(t-t_{j}\right) & =0 & & t<t_{b} \\
& =\frac{1}{\left(t_{e}-t_{b}\right)} & & t_{b} \leq t \leq t_{e} \\
& =0 & & t>t_{e}
\end{aligned}
$$

Using this failure rate function in Eqn (2.62) gives

$$
\begin{aligned}
& R(t)=0 \quad t<t_{b} \\
& =\frac{1}{\left(t_{e}-t_{b}\right)} \int_{t_{b}}^{t} r(t-\tau) d \tau \quad t_{b} \leq t \leq t_{e} \\
& =\frac{1}{\left(t_{e}-t_{b}\right)} \int_{t_{b}}^{t_{e}} r(t-\tau) d \tau \quad t>t_{e}
\end{aligned}
$$

c) Normal distribution failure rate.

If the container failure rate follows a normal distribution, the distribution function is:

$$
f(t)=\frac{1}{\sigma \sqrt{2 \pi}} e^{-(t-\mu)^{2} /\left(2 \sigma^{2}\right)}
$$


where $\sigma$ is the standard deviation and $\mu$ is the mean value for failure times.

Using this distribution, the release rate becomes

$$
R(t)=\frac{1}{\sqrt{2 \pi}} \int_{0}^{t} \frac{r(t-\tau)}{\sigma} e^{-(t-\mu)^{2} /\left(2 \sigma^{2}\right)} d \tau
$$

Introducing a change of variables, $\tau^{\prime}=(\tau-\mu) / \sigma$ leads to the following expression for release rate.

$$
R(t)=\frac{1}{\sqrt{2 \pi}} \int_{-\mu / \sigma}^{(t-\mu) / \sigma} r\left(t-\sigma \tau^{\prime}-\mu\right) e^{-\tau^{\prime 2} / 2} d \tau^{\prime}
$$

This distribution may not be an appropriate choice when the standard deviation is close to the mean. When this occurs, the model may predict a large fraction of containers to fail upon emplacement.

For example, assume a mean value of 40 years and a standard deviation of 20 years. In this case, the distribution would predict $2.2 \%$ of the containers fail before time, $\mathrm{t}=0$. Thus integration from $t=0$ out to $t=\infty$ would predict failure of only $97.8 \%$ of the containers. This can be treated by using another type of distribution (e.g. log normal) or by declaring that $2.2 \%$ of the containers fail at emplacement.

\subsubsection{Combination of DUST-MS release models with distributed failure times}

In DUST-MS release from a waste form is governed by one of four processes; diffusion, dissolution, rinse with partitioning, and solubility. The user controls the choice of the release model for each container. The user also controls the choice of container failure model and can select instantaneous, uniform, or guassian failure distribution. Based on the model selected, the code uses the appropriate equation ( 2.64 for instantaneous failure rate, 2.65 for uniform failure rate, and 2.69 for Gaussian failure distribution) with the appropriate release model. Ingrowth of radionuclides is calculated for all models.

\subsubsection{Selection of Release Models}

As part of this program an evaluation of disposal data has been made [Cowgill, 1992a; Sullivan, 1991; Cowgill, 1992]. These reports discuss the distribution of radioactivity by waste class, waste stream, and wasteform and provide a starting point for selection of the appropriate leaching model.

A major finding of these studies is that in general, there is limited data on releases from most low-level waste streams or wasteforms. The lack of data covers the major waste streams: dry active wastes, dewatered resins, and activated metals. There is substantial data on releases of $\mathrm{Cs}, \mathrm{Sr}$, and 
Co in cement solidified wastes. Unfortunately, only a small fraction of the activity of these radionuclides is in cement. For radionuclides that do exhibit substantial quantities in cement, e.g., C-14, Tc-99, U-238, and Ra-226, there is also limited data.

Due to the lack of data, it is recommended that the surface rinse model be used for all dry active wastes and dewatered resins. If a partition coefficient is available and can be justified it should be used. For cement solidified wastes, leaching data indicate that diffusion controlled release should be used. The choice of diffusion coefficient should be supported by data. If data does not exist, a conservative estimate for the diffusion coefficient is $10^{-6} \mathrm{~cm}^{2} / \mathrm{s}$. This is equivalent to a Leach Index of 6, the minimum allowed by the wasteform technical position [Lohaus, 1991]. For activated metals, a fractional release rate based on typical dissolution rates of the metals may be possible to justify.

For convenience, Table 2.1 presents a summary of the models selected for each of the four processes that influence release from the disposal facility.

\subsection{Transport Equation Initial and Boundary Conditions}

DUST-MS permits the user to define the initial condition of each contaminant at each spatial location in the modeled domain. Typically, the initial concentration is zero everywhere.

For each contaminant, DUST-MS permits a selection from four boundary conditions : Specified concentration:

$$
C_{i}\left(x_{b}, t\right)=f_{i}(t)
$$

Specified Total Flux: 


$$
\left(V_{d} C_{i}\left(x_{b}, t\right)-\theta D_{i} \nabla C_{i}\left(x_{b}, t\right)\right)=g_{i}(t)
$$

Specified Advective Flux:

$$
\left[V_{d} C_{i}\left(x_{b}, t\right)\right]=h_{i}(t)
$$

Specified Dispersive Flux:

$$
\theta D_{i} \nabla C_{i}\left(x_{b}, t\right)=j_{i}(t)
$$

Where the subscript $b$ on the spatial coordinate identifies the location as being part of the boundary. In Eqns.58 - 61 all boundary conditions are represented as a function of time. In addition, each contaminant can have its own unique set of boundary conditions. The values for the boundary condition functions, $\mathrm{f}_{\mathrm{i}}, \mathrm{g}_{\mathrm{i}}, \mathrm{h}_{\mathrm{i}}$, and $\mathrm{j}_{\mathrm{i}}$ are supplied through input as described in sections 6 and 7. Different boundary conditions can be used at each boundary. For example, for a contaminant, one boundary could have a specified total flux, while another boundary could have a specified concentration.

Selection of the boundary condition is an important aspect of defining the conceptual model of the system. The choice of boundary conditions will affect the model results. For example, consider a problem with vertically downward flow, selecting a zero concentration at the bottom boundary would lead to the maximum flux out of the system. However, it will not accurately calculate the concentrations at (or near) the boundary. Similarly, specifying a zero flux boundary condition will maximize the concentration at the boundary, but will not accurately represent the flux.

Typically, the boundary conditions specify zero flux out of the top of the system (i.e., no mass transfer out of the top of the facility and zero concentration at the bottom of the facility (i.e., maximum mass transfer out of the facility).

\subsubsection{Tabular Inlet Boundary Conditions}

Normally, DUST-MS has the boundary condition specified as a table of time versus the boundary condition (specified concentration, specified flux, etc.). This is discussed in detail later in this report (Sec 7.6). In addition, the user can specify that the upstream boundary condition can be a large table of values (up to 1000) for the specified mass flux. This is most frequently used when DUST-MS is run twice in succession. The first run is used to represent the disposal site and the vadose zone. As part of the first run, a set of files, one for each isotope, is created with the mass flux 
at specified locations. These files are called BOUND?.DAT with the ? being a number from 0 to 9 that represents the isotope in the simulation. If there are 10 istopes, the file Bound0.DAT represents that isotpe. By selecting a location at the bottom of the vadose zone, the file stores the flux in the aquifer. The second simulation uses the specified mass flux as the upstream boundary condition. This conserves mass between the vadose zone and the aquifer. Details of how to accomplish this can be found in Section 7.6. 


\begin{tabular}{|c|c|c|c|}
\hline Fluid Flow & Container Failure & $\begin{array}{l}\text { Wasteform } \\
\text { Release }\end{array}$ & Transport \\
\hline $\begin{array}{l}\text { Flow rate and moisture content within the } \\
\text { disposal facility evaluated using a table } \\
\text { look-up. }\end{array}$ & $\begin{array}{l}\text { Each representative container may have } \\
\text { different properties, i.e., } \\
\text { a) Time to failure. } \\
\text { b) Partial failure due to localized effects } \\
\text { (e.g., pitting). Early water access. } \\
\text { (FD Model) } \\
\text { Distributed Failure (DUSTMS-D) } \\
\text { Each representative control volume can } \\
\text { fail its containers in three ways } \\
\text { a) Instantaneous at a specified time } \\
\text { b) Uniformly distributed between a } \\
\text { failure start and end time } \\
\text { c) Gaussian distribution based on a } \\
\text { mean value and a standard } \\
\text { deviation. } \\
\text { The uniform and gaussian models can } \\
\text { also specify a fraction of the containers to } \\
\text { fail on emplacement. }\end{array}$ & $\begin{array}{l}\text { Each representative wasteform may have } \\
\text { different properties. Release } \\
\text { characteristics are nuclide specific. } \\
\text { Models account for: } \\
\text { a) Surface rinse with partitioning (Lab } \\
\text { trash, contaminated soils, DAW) } \\
\text { b) Diffusion (solidified wastes) - } \\
\text { Analytical solutions to the diffusion } \\
\text { equation assuming zero boundary } \\
\text { concentration (maximum release } \\
\text { rate). Release is geometry } \\
\text { dependent, models are provided for } \\
\text { both cylindrical and rectangular } \\
\text { wasteforms. (FD Model) } \\
\text { c) Uniform (activated metals) - } \\
\text { Constant release rate. } \\
\text { d) Solubility limited release. } \\
\text { (FD Model) } \\
\text { DUSTMS-D } \\
\text { Convolution is used to couple wasteform } \\
\text { release rates with the time distributed } \\
\text { container failures. }\end{array}$ & $\begin{array}{l}\text { Transport parameters such as the } \\
\text { retardation coefficient are nuclide } \\
\text { specific. } \\
\text { One-dimensional finite difference model. } \\
\text { Model that includes source terms (e.g. } \\
\text { wastes) and radioactive decay and } \\
\text { ingrowth... }\end{array}$ \\
\hline
\end{tabular}




\section{PROCEDURE FOR CALCULATING RELEASES USING DUST-MS}

The preceding chapter describes the models selected for estimating the source term but does not provide a clear indication of the steps needed to take the raw data, transform the data into the form required by the models, input the data and estimate the source term. Figure 3.1 is a schematic diagram that outlines this procedure.

The first step in this process is to compile the inventory data for the radionuclides of interest. Three factors that are determined before the waste is emplaced in a disposal facility figure prominently in determining release. These are the radionuclide's waste stream, wasteform, and container. A radionuclide contained in an activated metal will be released at a much different rate than the same radionuclide that exists as a surface contaminant on lab trash. Similar remarks apply for releases from different wasteforms. The container will control the time that release begins and for localized failure, the amount of water that accesses the waste.

Work to determine the feasibility of characterizing the radionuclide inventory based on the three parameters listed above has been done. This effort, based on commercial disposal data from 1987 through 1989 [Roles, 1990], will determine the activity fraction of the waste streams contained in various wasteform and container types (e.g. HIC's, carbon steel, etc.). Discussion of the most important waste stream/wasteform/container systems in terms of activity are provided in [Sullivan, 1991b; Cowgill, 1992; Cowgill, 1992a].

Due to the large number of possible waste stream/wasteform/container combinations it will not be possible, nor even desirable, to model each of these systems individually. Therefore, many of the combinations that do occur should be lumped together to form a "representative" wasteform/container systems. This grouping should be performed to handle the most important wasteforms in terms of release. After the "representative" systems have been identified, appropriate container degradation and wasteform release models and parameters need to be determined.

Using the flexibility of the source term model, it will be possible to specify different release models and parameters for each waste stream/wasteform combination. For example, ion exchange resins solidified in cement may be assumed to follow diffusion-controlled release with one diffusion coefficient while evaporator bottoms solidified in cement may be given another, and activated metals may be assumed to follow dissolution controlled release with a constant release rate. Similarly, for two identical waste stream/wasteforms in two separate containers, the predicted release can be different due to different container properties.

The inventory, container degradation and wasteform release parameters are input into the source term model as schematically depicted in Fig 3.1. These parameters along with the radionuclide specific parameters (e.g. half-life, solubility limit, etc.), transport parameters, water flow parameters (velocity and moisture content), initial conditions, and boundary conditions (finite difference model only) fully describe the problem. 


\title{
Source Term Calculation
}

\section{Data Evaluation}

\author{
Release Rate
}

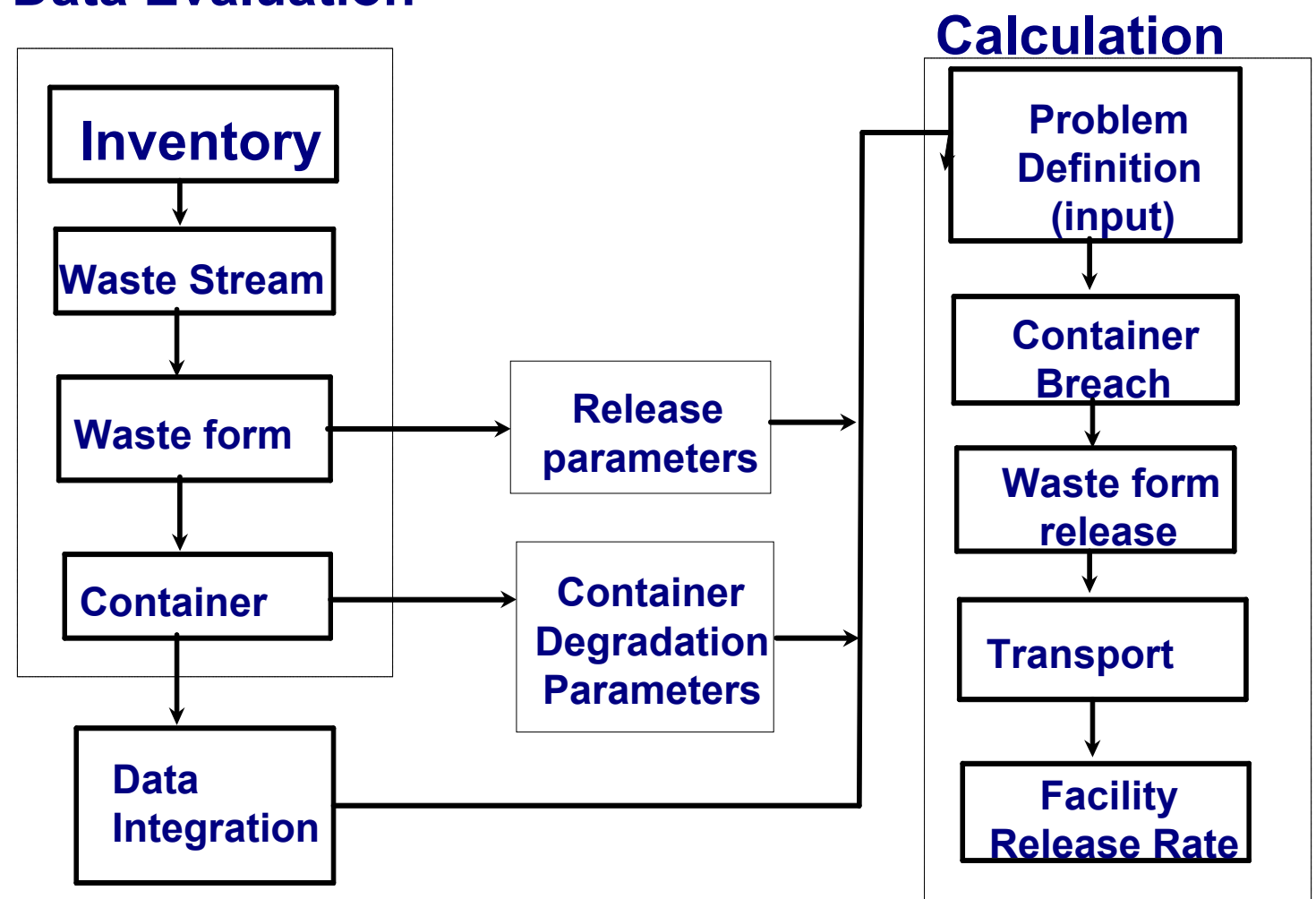

Calculation

Figure 3-1 Flowchart of the procedure used to take waste stream/wasteform/container inventory data and define the necessary input parameters to estimate the release rate from a disposal facility.

In general, the choice of input parameters used by the DUST-MS code must be justified. Justification can come from experimental data, that is, use measured diffusion coefficients to predict wasteform release, from the use of more sophisticated computer codes, or well-documented expert judgement. For example, the MCMC model in the DUST-MS code assumes a constant flow rate through the disposal unit. In selecting the flow rate, a two-dimensional computer code that predicts unsaturated flow such as VAM2D could be used as a basis for determining this parameter. In any case documentation of the basis for the use of an input variable should be supplied with the results of any simulation. The potential for misusing the simple models through improper choice of input data is large.

Once the data has been selected, an input deck for the DUST-MS code must be created. To facilitate this process, the program DUSTIN was written. DUSTIN is a menu-driven code that prompts the user for the input required by DUST. DUSTIN allows the user to create an entire input 
deck or, modify an existing input deck. Modifications can be individually made to every single input parameter. Use of DUSTIN is described in Chapter 6. DUSTIN was written for DOS based systems. Refinements to DUSTIN were made to create the pre-processor DUSTWIN. This processor works on Windows machines and provides a visual interface for creating input files.

After creating an input file, the calculation is ready to proceed. For the MCMC model which relies on an analytical solution, the predicted release is calculated at the times specified through input. For the finite difference model, the predicted release is obtained through solving the differential equation describing release and transport through the disposal facility at a fixed time, incrementing the time and repeating the procedure until the problem is finished.

The output of these models will be the release rate from the disposal unit as a function of time. This output will be stored in tabular form for use with performance assessment codes, such as PAGAN [Chu, 1991], that predict the transport of radionuclides through the unsaturated zone to the aquifer and ultimately to a receptor.

If requested by the code user, DUST-MS creates output files named TRCCND?.DAT and TRCFXD?.DAT. These files contain the concentration at specified locations and the flux and mass release at specified locations as a function of time. If the FD transport model is used, the file CONCNT.DAT is written. This file contains the concentration at every location at the times requested in the primary output file. The program GRAFMS.EXE can plot each of these three files on a video display unit. The use of GRAFMS is described in Chapter 9. The procedure to be followed in creating an input deck, running the DUST code and analyzing the output is presented in Figure 3.2. 


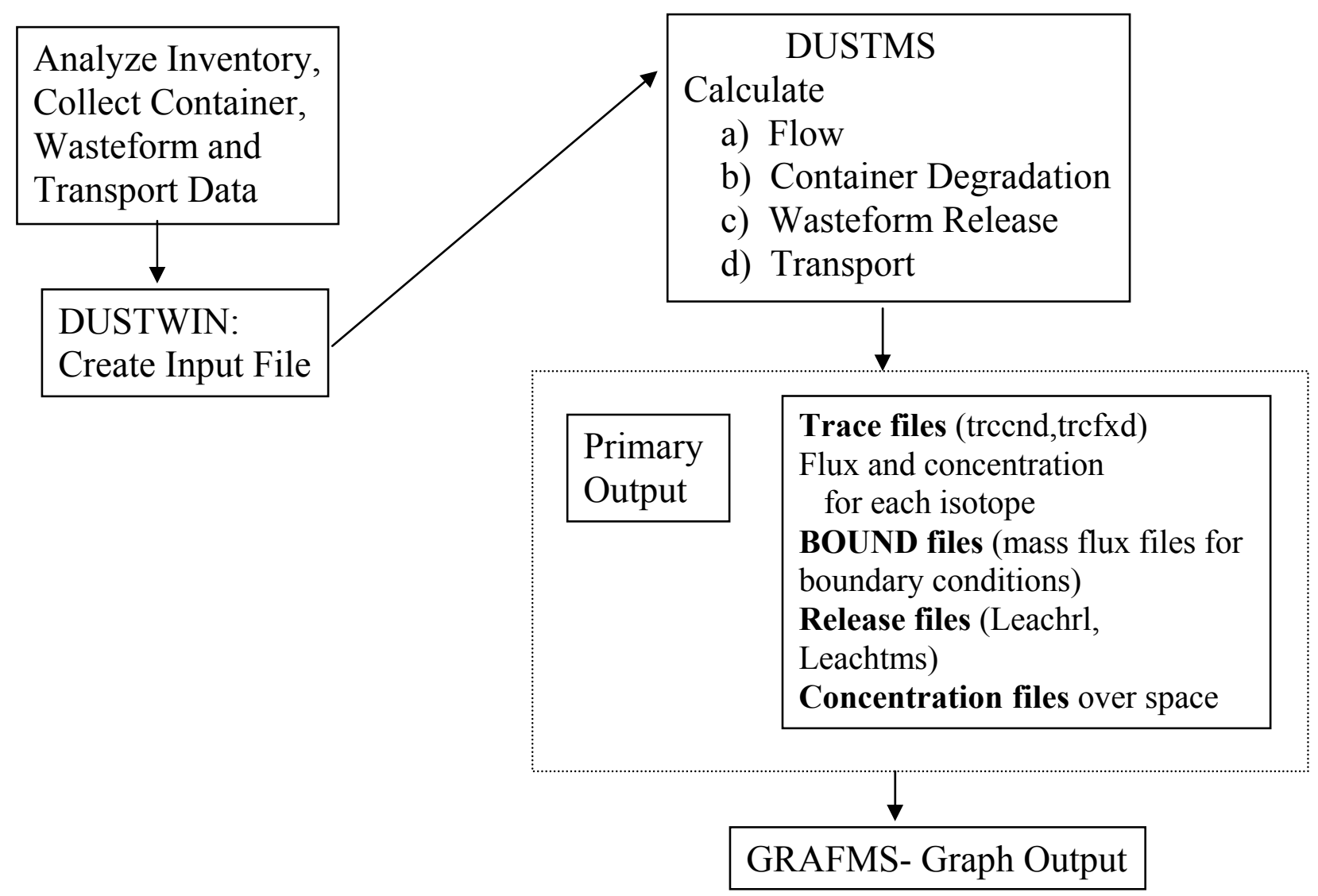

Figure 3- 2 Schematic outline of the procedure used to analyze release from a LLW disposal facility using the DUSTMS code package. 


\section{APPLICABILITY AND LIMITATIONS OF THE DUST_MS CODE}

The DUST-MS code models release and transport of a single radioactive contaminant through a low-level waste disposal facility in one spatial dimension. The source for transport is the contained radioactive wastes emplaced in the facility. Releases are a function of the container performance (time to failure) and wasteform performance (release rate). Each container may be assigned a unique time to failure and wasteform release rate parameters. This permits the flexibility to model a wide range of problems related to a waste disposal facility. These include determining the effects of the following parameters on release from the facility:

a) infiltration (flow rate);

b) container performance;

c) wasteform release rates; and

d) soil transport properties (retardation).

The DUST-MS code can be applied to a wide range of problems pertaining to low-level waste disposal ranging from lysimeter studies in which there is only a single soil and no waste container to below ground vaults with caps, multiple containers and wasteforms, engineered structures and backfill. However, due to the simplifications used in developing the models, the validity of the predicted results depends quite heavily on the input data, as discussed in Chapter 3. Many parameters in DUST (for example, water flow rate and container failure time) are determined within the code directly from user supplied input and not from first principles. Therefore, justification of the choice of input parameters is a critical aspect in developing confidence in DUST predictions.

\subsection{Limitations}

In developing the DUST-MS code, due to lack of mechanistic data and in order to limit the problem size to make the code executable on small desktop systems within a reasonable time, a number of assumptions were invoked. These assumptions may make use of the code inappropriate under certain conditions. In developing the MCMC model, a number of additional assumptions are used as compared to the FD model. The implication of these assumptions are discussed separately below. A list of the limitations follows.

(1) The DUST-MS code simulates only one spatial dimension. It therefore assumes a uniform geometry in the other two dimensions. This approximation will be best near the center plane of the facility where edge effects are minimized. The lack of spatial resolution may cause problems near special features such as drains or fractures in engineering structures. Modeling the entire facility such that the bottom boundary has the 
properties of a drain will overestimate release, while modeling the facility as being completely intact may underestimate releases. 
(2) The DUST-MS code models flow through a porous medium. Fracture flow, which may become significant at high relative moisture contents, through engineered barriers is not considered.

(3) Water flow and container failure time are not calculated from first principles in DUSTMS. They are determined through input. The user must justify the values used for these parameters.

(4) Biodegradation can produce radioactive gases, e.g. tritiated methane, 14CO2, or 14CH4. As a single species code, release in the gaseous and aqueous phase can not be handled simultaneously. An ad hoc procedure which partitions the inventory into the gaseous and aqueous phases may be used provided the code is run twice, once with the appropriate inventories and flow parameters for gaseous release and once for aqueous release. Work on estimating the partitioning between the gaseous and aqueous phases has been recently initiated.

(5) In modeling only a single nuclide, care must be taken to insure that solubility limits are not exceeded if other species containing that element exist. The solubility limit used in the DUST code applies to the modeled nuclide only. If the element exists in more than one nuclide, the solubility limit must be decreased to account for this problem. For example, if the DUST code were used to model U-238 and solubility limits are used, this solubility limit should reflect the presence of other uranium species.

(6) DUST-MS does not model changes in chemistry. The chemistry of the disposal facility is modeled through the distribution coefficient. Changes in this parameter due to changes in $\mathrm{pH}$, Eh and competition with other ions for sorption sites are not considered.

(7) In the FD model, the diffusion release subroutine is independent of the concentration in solution and solubility limits. This is a result of using the analytical solution based on a boundary condition of zero concentration at the wasteform-solute interface.

(8) In the MCMC model, the Darcy velocity must remain constant at all times. In the FD model, the Darcy velocity is calculated from a table of velocity versus time.

(9) In the MCMC model localized container failure is not modeled.

(10) In the MCMC model the distribution coefficient and moisture content must be constant throughout the facility and in time. The FD model permits these parameters to vary spatially.

(11) In the MCMC model release mechanisms are limited to the uniform release and rinse release without a wasteform partitioning factor. The FD model allows a partitioning factor and models diffusion controlled release. 
(12) As shown in the development of the equations, both the MCMC and FD models use upwind differencing to model the advection term. Upwind differencing insures that information is only advected downstream, however, it leads to numerical dispersion. The value of the numerical dispersion coefficient, $\mathrm{D}_{\mathrm{n}}$, in the FD model is [Roache, 1976]:

$$
D_{n}=\frac{V_{d} \Delta x}{2}(1-C)
$$

where $\mathrm{V}_{\mathrm{d}}$ is the Darcy velocity, $\Delta \mathrm{x}$ is the size of the finite difference node, $\mathrm{C}$ is the dimensionless Courant number, $\mathrm{V}_{\mathrm{d}} \Delta \mathrm{t} / \Delta \mathrm{x}$, and $\Delta \mathrm{t}$ is the time step size. In the limit as the time step size approaches 0 , the solution to the FD equation approaches the solution of the original differential equation modified due to numerical dispersion. In this case, the Courant number goes to zero and the numerical dispersion coefficient becomes:

$$
D_{n}=\frac{V_{d}}{2} \Delta x
$$

Heuristically, this can be viewed as the numerical dispersion coefficient for the MCMC model which uses an analytical solution. Numerical studies comparing the FD model with zero mechanical dispersion and diffusion with the MCMC model support this heuristic estimate of numerical dispersion for the MCMC model.

Recalling that the mechanical dispersion term from Eqn (2.1) is:

$$
\mathrm{D}=\mathrm{V}_{\mathrm{d}} \mathrm{a}_{\mathrm{t}}
$$

it is seen that the numerical dispersivity is $\Delta \mathrm{x} / 2$. Therefore, if the mesh size is more than twice as large is the mechanical dispersivity value, numerical dispersion will dominate. From the numerical dispersion expression it is clear that it can be minimized through taking small nodes. As a rule of thumb, the mechanical dispersion is typically $1 / 10$ to $1 / 100$ of the scale of the modeled domain. Therefore, $\Delta x$ should be much smaller than $1 / 5$ to $1 / 50$ of the modeled domain to insure that numerical dispersion is unimportant.

(13) Although conceptually, modeling gas flow through the facility is similar to modeling water flow, there is extremely little data on production of radioactive gases or expected flow rates. Therefore, the user must be extremely careful when selecting these values.

Radionuclides disposed of in gaseous form are expected to release quickly after container failure. For adsorbing gases it is expected that release would be controlled by a partitioning factor. Gases can also be formed by biodegradation, for example, tritiated methane, ${ }^{14} \mathrm{CO}_{2}$, or in the case of radon, through radioactive decay of radium. Few data 
exists on the formation of radioactive gases in a disposal facility. However, tritiated methane, ${ }^{14} \mathrm{CO}_{2},{ }^{14} \mathrm{CO}$, and other radioactive gases have been detected at the closed disposal sites at Sheffield [Streigel, 1985] and West Valley [Kunz, 1982; Matuszek, 1983]. While it is likely that the better disposal techniques (concrete vaults, no wooden or cardboard containers, solidification of the wastes in cement, etc.) planned for the new facilities may lead to less organic material, many of the wastes contain significant amounts of organics. This is particularly true for ${ }^{14} \mathrm{C}$ wastes [Gruhlke, 1986]. Therefore, releases caused by biodegradation cannot be dismissed at this time.

As a final caveat, uncertainties in the input data are often the most significant limitation in many models and codes. Frequently, many input parameters are not accurately known or available. Users of the DUST-MS code should be constantly aware of the limitation imposed by the quality of the input data. 


\section{VERIFICATION PROBLEMS}

It is important to verify that DUST-MS can solve the types of problems for which it is intended. Verification is critical to building confidence in the reliability of the computer model. Verification is usually accomplished through comparison with other codes or known analytical solutions. Both approaches are used here.

Successful code verification does not imply that the model can or will be successfully applied to any particular problem. It is the job of the code user to demonstrate that the models incorporated into DUST-MS capture the major physical and chemical processes that influence release from the disposal facility, that the data selected to represent the system accurately reflect the expected values, and the code is properly used (i.e., time step selection, element size, application of the initial and boundary conditions, proper translation of the physical model into a form amenable to solution by computer, etc.).

Even when properly applied, DUST-MS, in all likelihood, will not be able to reproduce the actual release rates that will eventually occur with a high degree of accuracy. The causes for the discrepancies are the long time frame (hundreds or thousands of years) in most simulations, the complex, heterogeneous nature of the natural and engineered physical setting, the variability in wastes and container, and lack of understanding of the physical and chemical processes that control release and transport. These limitations apply to all models. DUST-MS provides an excellent method for evaluating the interaction between several processes that are believed to control release from a disposal site.

DUST-MS is an extension of the DUST code [Sullivan, 1991] and, as such, the waste form leaching and container degradation models have undergone extensive verification studies previously [Sullivan, 1989, 1991]. Verification test cases were performed to demonstrate that in the absence of chain decay the DUST-MS model could reproduce the results generated by DUST. The container degradation, waste form leaching, and transport models were all tested independently. For presentation in this report, verification problems focused on the improvements to the original DUST code which permit solution of multiple species in a single simulation. Two major classes of problem were investigated: multi-species transport with chain decay and release of contaminants from the waste form when ingrowth occurs due to radioactive decay. The results of these studies are presented in the following sections.

\subsection{One-Dimensional Transport of a Single Three-Member Decay Chain}

To demonstrate that DUST-MS can accurately simulate transport, a one-dimensional test problem for a three-member decay chain was simulated. This problem contained a boundary source for the three members of the chain. At the left boundary, $x=0$, the incoming flux is the Darcy velocity multiplied by the concentration of the radionuclide of interest. For the first member of the chain, the initial concentration is unity and this changes in time due to exponential decay. The 
concentration of the second and third members of the chain are calculated using Bateman's equation [Bateman, 1910] with the assumption that their initial concentration is zero. The time dependence of the boundary condition was simulated through tabular input of the boundary condition flux values at specified times, Table 5.1. Linear interpolation was used for times not in the table. For convenience, the concentration at the boundary that corresponds to the flux in Table 5.1, is also presented in Table 5.1 The initial conditions were zero concentration at all locations for all radionuclides being simulated. The distance simulated in this problem was 70 meters. This distance is large enough such that during the problem simulation time, 273 years, essentially no material reached the right boundary. An analytical solution for this problem on a semi-infinite domain exists [Coats and Smith, 1964]. For comparison purposes, the contaminant and material specific transport parameters for this test problem were chosen to match with those reported by Huyakorn [Huyakorn, 1989]. These parameters are reported in Table 5.2.

The decay chain in this problem assumes that species one always decays to species two which always decays to species three. Branching of the decay chain is not considered. The values of the half-lives in Table 5.2 for the three contaminants are similar to those of Am-241, Pu-241, and $\mathrm{Pu}-240$. These three contaminants do not form a decay chain (e.g., Pu-241 decays to Am-241 which decays to Np-237). The half-lives were chosen to reflect a variety of decay rates over the time scale of interest (hundreds of years). The test problem selected the half-life of the first member of the chain to be short enough to insure that decay would occur and long enough such that it would not all decay. The contaminants are referred to as species 1,2, and 3 to emphasize that this problem does not represent a real decay chain.

\section{Table 5.1 Incoming Flux and Concentration at the Boundary for the Single Decay Chain Problem}

Flux versus time at the boundary (values used for the boundary conditions in the numerical simulations). Flux values are in $\left(\mathrm{g} / \mathrm{cm}^{2} / \mathrm{s}\right)$.

\begin{tabular}{lcccc}
\hline \multirow{2}{*}{ Contaminant } & \multicolumn{4}{c}{ Time(yrs) } \\
\cline { 2 - 5 } & 0 & 100 & 200 & 300 \\
\hline 1 & $2.3110^{-4}$ & $1.9710^{-4}$ & $1.6810^{-4}$ & $1.4310^{-4}$ \\
2 & 0.0 & $4.4910^{-6}$ & $6.3010^{-6}$ & $6.9010^{-6}$ \\
3 & 0.0 & $2.1610^{-6}$ & $1.8310^{-5}$ & $3.5610^{-5}$ \\
\hline
\end{tabular}

Concentrations versus time at the boundary. Concentration values are in $\mathrm{g} / \mathrm{cm}^{3}$.

\begin{tabular}{ccccc}
\hline \multirow{2}{*}{ Contaminant } & \multicolumn{4}{c}{ Time $(\mathrm{yrs})$} \\
\cline { 2 - 5 } & 0 & 100 & 200 & 300 \\
\hline 1 & 1.0 & .852 & 0.725 & 0.619
\end{tabular}




\begin{tabular}{lllll}
2 & 0 & 0.031 & 0.026 & 0.022 \\
3 & 0 & 0.116 & 0.247 & 0.353 \\
\hline
\end{tabular}

Table 5.2 Transport Properties Used in the Three-member Decay Chain Transport Problem

\begin{tabular}{lcc}
\hline & \multicolumn{2}{c}{ Radionuclide Dependent Properties } \\
\cline { 2 - 4 } Contaminant & \multicolumn{1}{c}{ T 2 (years) } & Distribution Coefficient $\left(\mathrm{K}_{\mathrm{d}}\right)$ \\
\hline 1 & 433 & 400 \\
\multicolumn{1}{c}{3} & \multicolumn{1}{c}{6540} & 400 \\
Contaminant Independent & Properties & 400 \\
\hline Moisture Content & 0.1 & \\
Darcy Velocity & $2.3110^{-4} \mathrm{~cm} / \mathrm{s}$ & \\
Bulk Density & $2.34 \mathrm{~g} / \mathrm{cm}^{3}$ & \\
Dispersion Coefficient & $259 \mathrm{~cm}$ & \\
Molecular Diffusion & $0 \mathrm{~cm}^{2} / \mathrm{s}$ & \\
Coefficient & & \\
\hline
\end{tabular}

The finite-difference solution was obtained on a uniform grid consisting of two elements 25 $\mathrm{cm}$ in length.. Flow was uniform in the X-direction and all transport properties were held constant over the modeled domain. The time step was one year for the entire simulation.

The distribution of the three contaminants as a function of distance at 273 years is presented in Figure 5.1. Comparison of the predicted concentrations with results obtained using VAM2D [Huyakorn, 1989] and BLT-MS shows excellent agreement [Table 5.3]. Differences between the three simulations are generally less than a few percent in the regions where the concentration is within two orders of magnitude of the maximum incoming concentration (unity). Comparisons at other times during the simulation showed similar differences in predicted results. The largest differences occurred for contaminant three. This may be due to the different treatment of radioactive decay. The VAM2D simulations used a central time difference procedure whereas, DUST-MS used a fully implicit backward difference solution procedure. VAM2D results were 
compared directly to the analytical solution and the results were judged to accurately represent transport [Huyakorn, 1989].

Figure 5.1 Comparison of DUST-MS and VAM2D Predicted Concentrations at 273 Years for a Three Member Decay Chain

Comparison of the analytical values for the boundary concentration to the results predicted

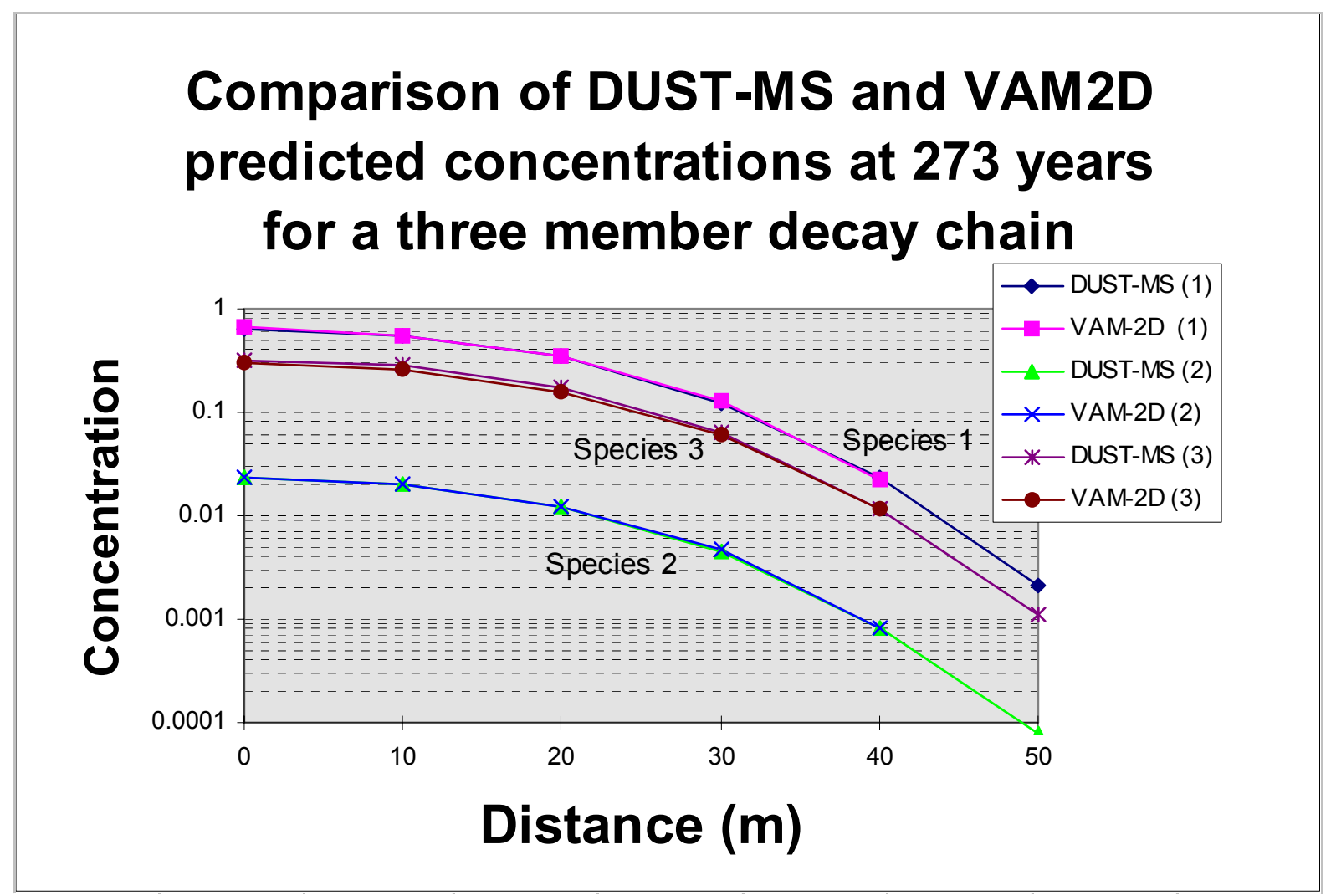

by the two codes indicates that in general DUST-MS provides slightly better estimates. The cause for this most likely lies in the different numerical treatment of the boundary conditions used in the two codes and the different mesh spacing and time steps. It has not been demonstrated that the DUST-MS methodology is superior to VAM2D for anything other than this test problem under these conditions. Both match the analytical solution within acceptable limits. The analytical value for the concentration at the boundary at 273 years for contaminant one is 0.646 which is between the values projected by the two codes ( 0.66 for VAM2D and 0.638 for DUST-MS). For contaminant two, the analytical boundary value is 0.0232 which again lies between the VAM2D $(0.0237)$ and the DUSTMS (0.0229) predictions. For contaminant three, the analytical value is 0.326 , which is slightly greater than the DUST-MS estimate, 0.320, and the VAM2D estimate, 0.305 . 


\subsection{One-dimensional Transport with Two Decay Chains}

To demonstrate the ability of DUST-MS to simulate multiple decay chains with branching, the preceding test problem was modified. The change in the problem involves the definition of the decay chain. In this simulation, species one decays to species two one-half the time and it decays to species three one-half of the time. Species two always decays to species three. The change in decay chain causes a change in the boundary conditions for the second and third species. The new boundary conditions are presented in Table 5.4. In the multiple decay chain model, all transport parameters are identical to the problem described in section 5.1 and Table 5.2

The change in the decay scheme reduces the source for species two by a factor of one-half. This is reflected in the boundary flux specified in Table 5.4. Therefore, predicted concentrations for species two should be one-half the value of the previous test case. The situation is more complex for species three. One-half of the decay from species one goes directly to species three while the other half goes to species two before decaying to species three. The decay directly to species three would indicate that the predicted concentrations should be higher than in the previous case when all of species one decayed to species two prior to becoming species three. Due to the short-half life of species two (15 years) this effect is not large. If the half-life of species two were much larger (hundreds or thousands of years), the effect of branching on species three would be much more pronounced. 
Table 5.3Comparison of VAM2D, BLT-MS and DUST-MS Results at 273 Years for the One-dimensional Single Decay Chain Problem

\begin{tabular}{|c|c|c|c|c|c|c|}
\hline \multirow[t]{2}{*}{ Species 1} & \multicolumn{6}{|c|}{ Distance (m) } \\
\hline & $\underline{0}$ & $\underline{10}$ & $\underline{20}$ & $\underline{30}$ & $\underline{40}$ & $\underline{50}$ \\
\hline BLT-MS & 0.639 & 0.560 & 0.347 & 0.124 & 0.0229 & 0.0021 \\
\hline DUST-MS & 0.639 & 0.559 & 0.350 & 0.128 & 0.0244 & 0.0023 \\
\hline VAM2D & 0.660 & 0.558 & 0.341 & 0.129 & 0.0220 & N/A \\
\hline Difference & -0.021 & 0.002 & 0.009 & -0.001 & 0.0024 & \\
\hline $\begin{array}{l}\% \\
\text { Difference }\end{array}$ & -3.2 & 0.2 & 2.6 & -0.8 & 10.9 & \\
\hline \multirow[t]{2}{*}{ Species 2} & \multicolumn{6}{|c|}{ Distance (m) } \\
\hline & $\underline{0}$ & $\underline{10}$ & $\underline{20}$ & $\underline{30}$ & $\underline{40}$ & $\underline{50}$ \\
\hline BLT-MS & 0.0229 & 0.020 & 0.0124 & 0.00446 & 0.000827 & $.7 \mathrm{E}-5$ \\
\hline DUST-MS & 0.0229 & 0.020 & 0.0126 & 0.0047 & 0.00088 & $8.3 \mathrm{E}-5$ \\
\hline VAM2D & 0.0237 & 0.020 & 0.0122 & 0.00465 & 0.000799 & $/ \mathrm{A}$ \\
\hline Difference & -0.00080 .0 & 0.0002 & -0.0004 & 0.000005 & $8 \mathrm{E}-5$ & \\
\hline $\begin{array}{l}\% \\
\text { Difference }\end{array}$ & -3.4 & 0.0 & 3.2 & 1.1 & 10.0 & \\
\hline \multirow[t]{2}{*}{ Species 3} & \multicolumn{6}{|c|}{ Distance (m) } \\
\hline & $\underline{0}$ & $\underline{10}$ & $\underline{20}$ & $\underline{30}$ & $\underline{40}$ & $\underline{50}$ \\
\hline BLT-MS & 0.32 & 0.281 & 0.175 & 0.0633 & 0.0119 & 0.0011 \\
\hline DUST-MS & 0.32 & 0.284 & 0.180 & 0.067 & 0.0129 & 0.0012 \\
\hline VAM2D & 0.305 & 0.259 & 0.160 & 0.062 & 0.0114 & N/A \\
\hline Difference & 0.015 & 0.025 & 0.020 & 0.005 & 0.0015 & \\
\hline $\begin{array}{l}\% \\
\text { Difference }\end{array}$ & 4.9 & 9.6 & 12.5 & 8.0 & 13.1 & \\
\hline
\end{tabular}




\section{Table 5.4 Incoming Flux and Concentration at the Boundary for the Two Decay Chain Problem}

Flux versus time at the boundary (values used for the boundary conditions in the numerical simulations). Flux values are in $\left(\mathrm{g} / \mathrm{cm}^{2} / \mathrm{s}\right)$.

\begin{tabular}{ccccc}
\hline \multirow{2}{*}{ Contaminant } & \multicolumn{4}{c}{ Time(yrs) } \\
\cline { 2 - 5 } & 0 & 91 & 200 & 300 \\
\hline 1 & $2.3110^{-4}$ & $1.9410^{-4}$ & $1.6810^{-4}$ & $1.4310^{-4}$ \\
2 & 0.0 & $3.7110^{-6}$ & $3.0110^{-6}$ & $2.5510^{-6}$ \\
3 & 0.0 & $2.7810^{-5}$ & $6.02210^{-5}$ & $9.83710^{-5}$ \\
\hline
\end{tabular}

Concentrations versus time at the boundary. Concentration values are in $\mathrm{g} / \mathrm{cm}^{3}$.

\begin{tabular}{ccccc}
\hline \multirow{2}{*}{ Contaminant } & \multicolumn{4}{c}{ Time $(\mathrm{yrs})$} \\
\cline { 2 - 5 } & 0 & 100 & 200 & 300 \\
\hline 1 & 1.0 & 0.852 & 0.725 & 0.619 \\
2 & 0 & 0.0155 & 0.013 & 0.011 \\
3 & 0 & 0.118 & 0.260 & 0.364 \\
\hline
\end{tabular}

The results from this simulation are presented in Table 5.5 and compared to the previous results from DUST-MS. The results are as expected. Species one predicted concentrations remain unchanged, species two concentrations have decreased by 2, and species three concentrations have increased by a few percent. A graphical display of the results generated by VAM2D is presented in [Huyakorn, 1989]. Comparison of the DUST-MS predicted concentrations to those in the graph showed agreement similar to that found for the problem described in section 5.1 (i.e., differences in predicted values less than $10 \%$ ). A detailed estimation of the differences could not be made because of the difficulties in obtaining two significant figures from the graph. 


\section{Table 5.5 Comparison of DUST-MS Results at 273 Years for Chain Decay and Chain Decay with Branching}

In the branched chain example, species one decays to species two one-half of the time and to species three the other half. Species two decays to species three all the time.

\begin{tabular}{|c|c|c|c|c|c|c|}
\hline \multirow[t]{2}{*}{ Species 1} & \multicolumn{6}{|c|}{ Distance (m) } \\
\hline & 0 & 10 & 20 & 30 & 40 & 50 \\
\hline Single chain & 0.639 & 0.559 & 0.350 & 0.128 & 0.0244 & 0.00233 \\
\hline $\begin{array}{l}\text { Branched } \\
\text { chain }\end{array}$ & 0.639 & 0.559 & 0.350 & 0.128 & 0.0244 & 0.00233 \\
\hline \multirow[t]{2}{*}{ Species 2} & \multicolumn{6}{|c|}{ Distance (m) } \\
\hline & 0 & 10 & 20 & 30 & 40 & 50 \\
\hline Single chain & 0.0229 & 0.020 & 0.0126 & 0.00447 & 0.00088 & $8.3 \mathrm{E}-5$ \\
\hline $\begin{array}{l}\text { Branched } \\
\text { chain }\end{array}$ & 0.01144 & 0.010 & 0.00628 & 0.00223 & 0.00044 & 4.18E-5 \\
\hline \multirow[t]{2}{*}{ Species 3} & \multicolumn{6}{|c|}{ Distance (m) } \\
\hline & 0 & 10 & 20 & 30 & 40 & 50 \\
\hline Single chain & 0.32 & 0.284 & 0.180 & 0.067 & 0.0129 & 0.00125 \\
\hline $\begin{array}{l}\text { Branched } \\
\text { chain }\end{array}$ & 0.364 & 0.291 & 0.181 & 0.067 & 0.0130 & 0.00126 \\
\hline
\end{tabular}

\subsection{Ingrowth of Progeny Prior to Release from the Waste Form}

For radionuclides that are members of a decay chain, their inventory will change in time prior to release from the waste form. A test problem was devised to demonstrate that DUST-MS could accurately simulate ingrowth in the waste form. In the test problem, there are two containers with identical waste forms. The first container fails at emplacement, $($ time $=0)$ and the second fails after 100 years. In DUST-MS for the analytical release models, the mass assigned for release due to one mechanism is independent of the other mechanisms (i.e., mass apportioned to diffusion must be released via diffusion). 


\subsubsection{Rinse Model}

The rinse model releases all of its available inventory during the first time step after container breach. Therefore, it provides an excellent method of testing the DUST-MS calculation of ingrowth in the waste form because the analytical inventory prior to release can be calculated using the Bateman equations. In this test problem, the three contaminants from the previous example were used. The initial inventory of species one was selected to be unity while the initial inventory of species two and three was zero. Therefore, any inventory of species two and three is a result of radioactive decay and ingrowth. The half lives are given in Table 5.2.

The results of this test are presented in Table 5.6. For the container that failed at time 0 , species one released the entire inventory as expected. At 100 years, the time of failure for the second container, the predicted results matched the analytical results to three significant figures.

Table 5.6 DUST-MS Predicted Rinse Release Compared to Analytical Rinse Release (Analyt)

\begin{tabular}{ccccccc}
\hline Container & \multicolumn{2}{c}{ Species 1 } & \multicolumn{2}{c}{ Species 2 } & \multicolumn{2}{c}{ Species 3 } \\
\cline { 2 - 6 } $\begin{array}{c}\text { Failure } \\
\text { Time (yrs) }\end{array}$ & DUST-MS & Analyt & DUST-MS & Analyt & DUST-MS & Analyt \\
\hline 0 & 1.0 & 1.0 & 0 & 0 & 0 & 0 \\
100 & 0.852 & 0.852 & 0.0309 & 0.309 & 0.116 & 0.116 \\
\hline
\end{tabular}

\subsubsection{Diffusion Model}

DUST-MS contains two classes of waste form leaching diffusion release models. The first class contains analytical solutions for diffusion from a finite-sized cylinder or plane with a uniform initial concentration and zero concentration at the boundary between the outside edge of the waste form and the contacting solution. When diffusion can be shown to be the dominant release mechanism, these models can be an excellent approximation to release from solidified waste forms. The second class contains a one-dimensional finite difference representation of diffusion through a solidified medium. This procedure is more general and permits solution feedback effects (non-zero concentrations at the boundary, e.g., solubility limited), and non-uniform production due to decay to be addressed in the framework of diffusion controlled release. These models are discussed in detail in section 2.32. and 2.3.5. 
When ingrowth occurs, the assumptions used to generate the analytical solution are no longer met. The production of progeny depends on the distribution of the precursor radionuclide. Even if the parent radionuclide begins with a uniform distribution in the waste form, due to the diffusion process, in time, the parent will have a spatially dependent distribution which implies a spatially dependent production rate of the progeny. To overcome the lack of an analytical solution for the case of a spatially varying source, the approximation is made that the spatial distribution of the source due to decay is identical to the spatial variation in concentration of the progeny in the waste form. In this case, the effect is to shift the entire concentration versus location curve in the waste form up to account for ingrowth. This approximation allows the analytical solution for diffusion release to be retained by simply adjusting the Ainitial concentration@. This approximation does not have a physical basis although it will conserve mass. In most cases, this approximation will tend to under predict releases from the waste form of the progeny species. If diffusion controlled release from the waste form is important, the use of the analytical diffusion models is not recommended when ingrowth occurs.

In contrast, the finite-difference leaching model accounts for the spatial variation in production rate as the problem evolves. Ingrowth is calculated within each finite difference volume used to represent the waste form. Therefore, if the parent and progeny have different diffusion rates this is automatically calculated as part of the solution procedure.

Several test problems were simulated to test the diffusion-controlled release leaching model when ingrowth occurs. The test problems considered three species. The initial inventory of species one was unity while the initial inventory of the second and third species was zero. In the first test problem, the diffusion coefficient of each species was $10^{-8} \mathrm{~cm}^{2} / \mathrm{s}$. The waste form was assumed to be represented by a plane with a half-width of $25 \mathrm{~cm}$. Two containers were considered, the first failed upon emplacement, the second failed after 100 years. The half-lives of the contaminants are those used previously and can be found in Table 5.2.

Table 5.7 contains the cumulative release after 273 years for the three species. For the first and third species the results of the two models are essentially identical. The amount of species two released is substantially higher for the finite-difference model as compared to the approximate analytical model. For species two, the difference between the two models is greatest for the container that failed at $\mathrm{t}=0$. 
Table 5.7 Comparison of Finite-Difference (PFD) and Approximate Analytical Model (AAM) Predictions for Cumulative Mass Release (CMR) from a Plane Waste Form with Each Species Having Identical Diffusion Coefficients in the Waste form.

\begin{tabular}{lcccc}
\hline & Species 1 & Species 2 & Species 3 & $\begin{array}{c}\text { Container } \\
\text { Failure } \\
\text { time }\end{array}$ \\
\cline { 2 - 5 } Model & CMR & CMR & CMR & \\
\hline PFD & 0.364 & $9.41 \mathrm{E}-3$ & 0.0443 & 0 \\
AAM & 0.365 & $1.71 \mathrm{E}-3$ & 0.0443 & 0 \\
PFD & 0.259 & $9.36 \mathrm{E}-3$ & 0.0635 & 100 \\
AAM & 0.259 & $4.77 \mathrm{E}-3$ & 0.0633 & 100 \\
\hline
\end{tabular}

A similar test problem was simulated for a cylindrical waste form with a $25 \mathrm{~cm}$. radius. The analytical diffusion model in cylindrical geometry also accounts for the finite height of the waste form. In this problem the height was set to a large value $(>5000 \mathrm{~cm})$ to insure that release occurred primarily in the radial direction. This allowed direct comparison with the finite-difference model which only simulates the radial direction. Other than the change in geometry, all other parameters were unchanged from the problem described above in this section.

Table 5.8 presents the cumulative release and mass available after 273 years for three species from a cylindrical waste form. The releases of the first species are much higher in this case. This is due to the geometry, more of the mass lies closer to the outside boundary in a cylinder of $25 \mathrm{~cm}$ radius as compared to a plane of $25 \mathrm{~cm}$ half-width. Thus, the average diffusion length is shorter and the release is higher. Again agreement between the analytical diffusion model and the finitedifference model is excellent for the first and thrid species. For the second species, the results were similar to those found for plane geometry. The finite-difference model predicts higher releases with the difference between the two models more pronounced as the diffusion time (time since breach) increases. 
Table 5.8 Comparison of Finite-difference (CFD) and Approximate Analytical Model (AAM) Predictions for Cumulative Fractional Release (CMR) from a Cylindrical Waste Form with Each Species Having Identical Diffusion Coefficients in the Waste Form

\begin{tabular}{lcccc}
\hline & Species 1 & Species 2 & Species 3 & $\begin{array}{c}\text { Container } \\
\text { Failure } \\
\text { time }\end{array}$ \\
\cline { 2 - 5 } Model & CMR & CMR & CMR & \\
\hline FD & 0.605 & 0.0149 & 0.063 & 0 \\
AAM & 0.609 & 0.0031 & 0.065 & 0 \\
FD & 0.447 & 0.0163 & 0.105 & 100 \\
AAM & 0.456 & 0.0089 & 0.106 & 100 \\
\hline
\end{tabular}

A similar test problem was simulated for a spherical waste form with a $25 \mathrm{~cm}$. radius. Other than the change in geometry, all other parameters were unchanged from the problem described above in this section.

Table 5.9 presents the cumulative release after 273 years for three species from a spherical waste form. The releases of the first species are much higher in this case as compared to either the plane or cylindrical geometry case.. This is due to the geometry, more of the mass lies closer to the outside boundary in a sphere of $25 \mathrm{~cm}$ radius as compared to a plane or cylinder with the principal dimension of $25 \mathrm{~cm}$. Thus, the average diffusion length is shorter and the release is higher. Again agreement between the analytical diffusion model and the finite-difference model is excellent for the first species. For the second and third species, the results were similar to those found for the other geometries. That is, the finite-difference model predicts higher releases of each species with the difference between the two models more pronounced as the diffusion time (time since breach) increases. 
Table 5.9 Comparison of Finite-difference (CFD) and Approximate Analytical Model (AAM) Predictions for Cumulative Mass Release (CMR) from a Spherical Waste Form with Each Species Having Identical Diffusion Coefficients in the Waste Form

\begin{tabular}{lcccc}
\hline & Species 1 & Species 2 & Species 3 & $\begin{array}{c}\text { Container } \\
\text { Failure } \\
\text { time }\end{array}$ \\
\cline { 2 - 5 } Model & CMR & CMR & CMR & \\
\hline FD & 0.757 & 0.017 & 0.066 & 0 \\
AAM & 0.761 & 0.0042 & 0.070 & 0 \\
FD & 0.581 & 0.021 & 0.131 & 100 \\
AAM & 0.585 & 0.012 & 0.133 & 100 \\
\hline
\end{tabular}

\subsubsection{Diffusion with Ingrowth}

To demonstrate that the finite-difference model can accurately simulate ingrowth due to radioactive decay, the following test problem was used. A two species decay chain was modeled with U-238 as the first member of the chain and Th-230 as the second member. Three spherical waste forms were modeled each with a radius of $25 \mathrm{~cm}$. The diffusion coefficient for U-238 was set to $10^{-26} \mathrm{~cm} 2 / \mathrm{s}$ in each waste form. The low diffusion coefficient and the long half-life of U-238 (4.47 $10^{9}$ years) provide an approximately constant source over the 1000 year time frame simulated. The diffusion coefficient for Th-230 was selected at $10^{-6}, 10^{-8}$, and $10^{-10} \mathrm{~cm}^{2} / \mathrm{s}$ for the three waste forms, respectively. The initial concentration of Th-230 in the waste form was zero while the initial mass of U-238 in each waste form was $10^{5}$ grams.

The analytical solution for the concentration within a sphere with a constant source and zero concentration at the boundary when diffusion is the only transport mechanism can be obtained by analogy with the heat conduction results found in Carslaw and Jaeger [Carslaw and Jaeger, 1959, p. 243]. The resulting concentration profile is:

$$
C(r, t)=\frac{Q_{0}}{6 D}\left(a^{2}-r^{2}\right)+\frac{2 Q_{0} a^{3}}{\pi^{3} D r} \sum_{n=1}^{\infty} \frac{(-1)^{n}}{n^{3}} \sin \left[\frac{n \pi r}{a}\right] e^{-n^{2} \alpha t}
$$

where $Q_{0}$ is the source per unit volume per unit time, $D$ is the diffusion coefficient, a is the radius of the waste form, $r$ is the position co-ordinate, and $\alpha$ is the time constant. In this problem, and 


$$
\begin{gathered}
Q_{0}=\frac{\lambda_{U} M_{0}}{\theta V_{w f}} \\
\alpha=\frac{\pi^{2} D}{a^{2}}
\end{gathered}
$$

where $\lambda_{U}$ is the decay constant for $U-238$, and $M_{o}$ is the initial mass of $U-238, \theta$ is the moisture content in the waste form and $\mathrm{V}_{\mathrm{wf}}$ is the volume of the waste form.

The flux out of the waste form can be obtained by differentiating the concentration expression, evaluating at $r=a$, and multiplying by the negative of the diffusion coefficient. The cumulative mass release (CMR) is obtained by integrating the product of the flux and waste form surface area over time. Performing these calculations yields:

$$
C M R=\lambda M_{0} t+\frac{6 \lambda M_{0}}{\pi^{2} \alpha} \sum_{n=1}^{\infty} \frac{(-1)^{n}}{n^{4}} e^{-\left(\alpha n^{2} t\right)}
$$

Equation 5.1 was evaluated at 500 and 1000 years at the ten locations corresponding to the finite difference locations used by DUST-MS. Equation 5.4 was evaluated at 500 and 1000 years for three diffusion coefficient values, $10^{-6}, 10^{-8}$, and $10^{-10} \mathrm{~cm}^{2} / \mathrm{s}$. Comparison of the DUST-MS results and analytical solution is presented in Table 5.10 for 500 years and Table 5.11 for 1000 years.

From Table 5.10 and 5.11 it is clear that DUST-MS reproduces the analytical solution for concentration to within $1 \%$ over the range tested. For the $D=10^{-6} \mathrm{~cm}^{2} / \mathrm{s}$ case, the steady state solution has been reached by 500 years and the concentration does not change between 500 and 1000 years. The $\mathrm{D}=10^{-8} \mathrm{~cm}^{2} / \mathrm{s}$ case, the solution is approaching steady-state after 1000 years. The test case with the smallest diffusion coefficient, $\mathrm{D}=10^{-10} \mathrm{~cm}^{2} / \mathrm{s}$, the solution has not come close to reaching steady-state even after 1000 years. The total mass produced after 1000 years is $1.55 \mathrm{E}-7$, therefore, in the higher diffusion cases, almost $100 \%$ of the mass is released. The DUST-MS estimate for the mass released is approximately $10 \%$ low for the $\mathrm{D}=10^{-10} \mathrm{~cm}^{2} / \mathrm{s}$ case. This is believed to be due to inaccuracies in the finite difference approximation at the boundary when the gradient is large in the last computational node as compared to in previous nodes.

Performing the same calculation with the analytical spherical release model yields a cumulative mass release at 1000 years of $1.5 \mathrm{E}-9,1.86 \mathrm{E}-8,1.07 \mathrm{E}-8$, for $\mathrm{D}=10^{-6}, 10^{-8}, 10^{-10} \mathrm{~cm}^{2} / \mathrm{s}$, respectively. Thus, the analytical model predicted mass release is ranges between two orders of magnitude low for the fastest diffusion coefficient to a factor of two low for the slowest diffusion coefficient. This demonstrates the inaccuracies that can arise in using the analytical models when ingrowth occurs. 
Table 5.10 Comparison of DUST-MS and Analytical Solution predicted concentrations and Cumulative Mass Release (CMR) at 500 years.

\begin{tabular}{lcccccc}
\hline & $\mathrm{D}=10^{-6}$ & \multicolumn{3}{c}{$\mathrm{D}=10^{-8}$} & $\mathrm{D}=10^{-10}$ & \\
\cline { 2 - 7 } $\begin{array}{l}\text { Distance } \\
(\mathrm{cm})\end{array}$ & Analyt & $\begin{array}{c}\text { DUST- } \\
\text { MSS }\end{array}$ & Analyt & $\begin{array}{c}\text { DUST- } \\
\text { MSS }\end{array}$ & Analyt & $\begin{array}{c}\text { DUST- } \\
\text { MSS }\end{array}$ \\
\hline 2.5 & $3.87 \mathrm{E}-14$ & $3.92 \mathrm{E}-14$ & $3.48 \mathrm{E}-12$ & $3.49 \mathrm{E}-12$ & $5.92 \mathrm{E}-12$ & $5.91 \mathrm{E}-12$ \\
5.0 & $3.75 \mathrm{E}-14$ & $3.78 \mathrm{E}-14$ & $3.39 \mathrm{E}-12$ & $3.37 \mathrm{E}-12$ & $5.92 \mathrm{E}-12$ & $5.91 \mathrm{E}-12$ \\
7.5 & $3.56 \mathrm{E}-14$ & $3.58 \mathrm{E}-14$ & $3.22 \mathrm{E}-12$ & $3.20 \mathrm{E}-12$ & $5.92 \mathrm{E}-12$ & $5.91 \mathrm{E}-12$ \\
10.0 & $3.28 \mathrm{E}-14$ & $3.30 \mathrm{E}-14$ & $2.99 \mathrm{E}-12$ & $2.97 \mathrm{E}-12$ & $5.92 \mathrm{E}-12$ & $5.91 \mathrm{E}-12$ \\
12.5 & $2.93 \mathrm{E}-14$ & $2.95 \mathrm{E}-14$ & $2.68 \mathrm{E}-12$ & $2.67 \mathrm{E}-12$ & $5.92 \mathrm{E}-12$ & $5.91 \mathrm{E}-12$ \\
15.0 & $2.50 \mathrm{E}-14$ & $2.52 \mathrm{E}-14$ & $2.30 \mathrm{E}-12$ & $2.29 \mathrm{E}-12$ & $5.92 \mathrm{E}-12$ & $5.91 \mathrm{E}-12$ \\
17.5 & $1.99 \mathrm{E}-14$ & $2.01 \mathrm{E}-14$ & $1.85 \mathrm{E}-12$ & $1.84 \mathrm{E}-12$ & $5.92 \mathrm{E}-12$ & $5.91 \mathrm{E}-12$ \\
20.0 & $1.41 \mathrm{E}-14$ & $1.42 \mathrm{E}-14$ & $1.32 \mathrm{E}-12$ & $1.32 \mathrm{E}-12$ & $1.17 \mathrm{E}-11$ & $5.88 \mathrm{E}-12$ \\
22.5 & $7.43 \mathrm{E}-15$ & $7.53 \mathrm{E}-15$ & $7.00 \mathrm{E}-13$ & $7.05 \mathrm{E}-13$ & $9.84 \mathrm{E}-12$ & $5.47 \mathrm{E}-12$ \\
24.37 & $1.93 \mathrm{E}-15$ & $1.96 \mathrm{E}-15$ & $1.83 \mathrm{E}-13$ & $1.83 \mathrm{E}-13$ & $3.82 \mathrm{E}-12$ & $2.59 \mathrm{E}-12$ \\
$\mathrm{CMR}$ & $7.73 \mathrm{E}-8$ & $7.74 \mathrm{E}-8$ & $5.87 \mathrm{E}-8$ & $5.85 \mathrm{E}-8$ & $8.51 \mathrm{E}-9$ & $7.76 \mathrm{E}-9$ \\
\hline
\end{tabular}


Table 5.11 Comparison of DUST-MS and Analytical Solution predicted concentrations and Cumulative Mass Release (CMR) at 1000 years.

\begin{tabular}{lcccccc|}
\hline & $\mathrm{D}=10^{-6}$ & \multicolumn{3}{c}{$\mathrm{D}=10^{-8}$} & $\mathrm{D}=10^{-10}$ & \\
\cline { 2 - 7 } $\begin{array}{l}\text { Distance } \\
(\mathrm{cm})\end{array}$ & Analyt & $\begin{array}{c}\text { DUST- } \\
\text { MS }\end{array}$ & Analyt & $\begin{array}{c}\text { DUST- } \\
\text { MS }\end{array}$ & Analyt & $\begin{array}{c}\text { DUST- } \\
\text { MS }\end{array}$ \\
\hline 2.5 & $3.87 \mathrm{E}-14$ & $3.92 \mathrm{E}-14$ & $3.84 \mathrm{E}-12$ & $3.87 \mathrm{E}-12$ & $1.18 \mathrm{E}-11$ & $1.18 \mathrm{E}-11$ \\
5.0 & $3.75 \mathrm{E}-14$ & $3.78 \mathrm{E}-14$ & $3.72 \mathrm{E}-12$ & $3.74 \mathrm{E}-12$ & $1.18 \mathrm{E}-11$ & $1.18 \mathrm{E}-11$ \\
7.5 & $3.56 \mathrm{E}-14$ & $3.58 \mathrm{E}-14$ & $3.53 \mathrm{E}-12$ & $3.54 \mathrm{E}-12$ & $1.18 \mathrm{E}-11$ & $1.18 \mathrm{E}-11$ \\
10.0 & $3.28 \mathrm{E}-14$ & $3.30 \mathrm{E}-14$ & $3.26 \mathrm{E}-12$ & $3.26 \mathrm{E}-12$ & $1.18 \mathrm{E}-11$ & $1.18 \mathrm{E}-11$ \\
12.5 & $2.93 \mathrm{E}-14$ & $2.95 \mathrm{E}-14$ & $2.91 \mathrm{E}-12$ & $2.92 \mathrm{E}-12$ & $1.18 \mathrm{E}-11$ & $1.18 \mathrm{E}-11$ \\
15.0 & $2.50 \mathrm{E}-14$ & $2.52 \mathrm{E}-14$ & $2.49 \mathrm{E}-12$ & $2.49 \mathrm{E}-12$ & $1.18 \mathrm{E}-11$ & $1.18 \mathrm{E}-11$ \\
17.5 & $1.99 \mathrm{E}-14$ & $2.01 \mathrm{E}-14$ & $1.98 \mathrm{E}-12$ & $1.99 \mathrm{E}-12$ & $1.18 \mathrm{E}-11$ & $1.18 \mathrm{E}-11$ \\
20.0 & $1.41 \mathrm{E}-14$ & $1.42 \mathrm{E}-14$ & $1.40 \mathrm{E}-12$ & $1.41 \mathrm{E}-12$ & $1.17 \mathrm{E}-11$ & $1.15 \mathrm{E}-11$ \\
22.5 & $7.43 \mathrm{E}-15$ & $7.53 \mathrm{E}-15$ & $7.39 \mathrm{E}-13$ & $7.46 \mathrm{E}-13$ & $9.84 \mathrm{E}-12$ & $9.87 \mathrm{E}-12$ \\
24.37 & $1.93 \mathrm{E}-15$ & $1.96 \mathrm{E}-15$ & $1.92 \mathrm{E}-13$ & $1.93 \mathrm{E}-13$ & $3.82 \mathrm{E}-12$ & $3.74 \mathrm{E}-12$ \\
$\mathrm{CMR}$ & $1.55 \mathrm{E}-7$ & $1.55 \mathrm{E}-7$ & $1.35 \mathrm{E}-7$ & $1.34 \mathrm{E}-7$ & $2.37 \mathrm{E}-8$ & $2.21 \mathrm{E}-8$ \\
\hline
\end{tabular}




\subsubsection{Uniform Degradation Model}

There are two major classes of solution procedure for the uniform degradation model. The analytical uniform degradation model, which in the absence of ingrowth and decay, projects a uniform time independent release rate until the entire inventory is released. For example, if the fractional degradation rate was 0.01 per year, $1 \%$ of the inventory would be released for 100 consecutive years after breach. This release is adjusted for decay and ingrowth as discussed in section 2.3.7.

The second class of degradation model is the finite-difference models, discussed in section 2.3.5. The finite-difference models can simulate either plane, cylindrical, or spherical geometry. In each case, the fractional degradation rate is multiplied by a characteristic length (width for plane geometry, radius for cylindrical or spherical geometry) to obtain a dissolution velocity. The release rate is the concentration in the waste form available for uniform degradation release multiplied by the dissolution velocity multiplied by the surface area to volume ratio of the waste form. For plane geometry, this reduces to an expression identical to the analytical model. For cylindrical and spherical geometry, the effects of curvature cause a time-dependent release rate. That is, the amount released is proportional to the surface area which is a function of the time-dependent radius. As the radius decreases due to dissolution, the surface area decreases and so does the release rate.

To test the dissolution models in DUST-MS the test problem described in the previous section was modified slightly. The problem again considers three species with the initial condition that species one has a unit inventory while species two and three are initially absent. The fractional degradation rate was set to 0.001 for all three species. Two container failure times, 0 and 100 years, were simulated. For the finite-difference models, the half-width was set to $25 \mathrm{~cm}$ for plane geometry and the radius was set to $25 \mathrm{~cm}$ for cylindrical geometry.

The results of this test problem are presented in Table 5.12. As expected, the analytical model and the plane-geometry finite difference model gave identical results for all three species. The cylindrical release model exhibits higher releases and the spherical geometry model exhibits the highest releases, .as expected.

To verify the accuracy of the models an analytical expression for the cumulative fractional release is obtained for the first member of the chain.

$$
Q=\int_{0}^{t} u L_{0} M_{o} e^{(-\lambda t)} \frac{A(t)}{V_{0}}
$$

Where $\mathrm{u}$ is the fractional degradation rate, $\mathrm{L}_{\mathrm{o}}$ is the characteristic length (width for plane geometry, radius for cylindrical or spherical geometry ), $\mathrm{M}_{\mathrm{o}}$ is the initial inventory in the waste 
form, $\lambda$ is the decay constant, $V_{o}$ is the volume of the waste form at emplacement, and $A(t)$ is the surface 
Table 5.12 Comparison of Plane Finite-difference (PFD), Cylindrical Finite Difference (CFD), Spherical

Finite Difference (SFD) and Analytical Model (AM)

Predictions for Cumulative Mass Release (CMR) due to dissolution of a waste form.

\begin{tabular}{lcccc}
\hline & Species 1 & Species 2 & Species 3 & $\begin{array}{c}\text { Container } \\
\text { Failure } \\
\text { time }\end{array}$ \\
\cline { 2 - 5 } Model & CMR & CMR & CMR & \\
\hline PFD & 0.222 & $7.48 \mathrm{E}-3$ & 0.0448 & 0 \\
AM & 0.222 & $7.38 \mathrm{E}-3$ & 0.0445 & 0 \\
CFD & 0.388 & 0.0129 & 0.073 & 0 \\
SFD & 0.513 & 0.0169 & 0.0892 & 0 \\
PFD & 0.129 & $4.85 \mathrm{E}-3$ & 0.0393 & 100 \\
AM & 0.129 & $4.90 \mathrm{E}-3$ & 0.0387 & 100 \\
CFD & 0.237 & $8.92 \mathrm{E}-3$ & 0.0798 & 100 \\
SFD & 0.328 & $1.232 \mathrm{E}-2$ & 0.0958 & 100 \\
\hline
\end{tabular}

area of the waste form. For plane geometry, $\mathrm{L}_{\mathrm{o}} \mathrm{A}(\mathrm{t}) / \mathrm{V}_{\mathrm{o}}$ is unity and the expression reduces to:

$$
Q_{p}=\frac{u M_{o}}{\lambda}\left[1-e^{(-\lambda t)}\right]
$$

For the container that fails at emplacement, evaluation of the above expression at 273 years yields a value of 0.22 as predicted by the DUST-MS analytical model and plane finite-difference model. For the container that fails at 100 years, $\mathrm{M}_{\mathrm{o}}$ is reduced to 0.85 (to account for decay prior to breach and $t$ is 174 years. In this case, the analytical release is 0.13 which agrees with the DUSTMS model predictions. The above relationship could be generalized by replacing $\mathrm{M}_{\mathrm{o}} \mathrm{e}^{(-\lambda \mathrm{t})}$ by the expression for the mass as a function of time as determined from the Bateman equations.

For cylindrical geometry, $\mathrm{L}_{\mathrm{o}}$ is the initial radius $\mathrm{r}_{\mathrm{o}}, \mathrm{V}_{\mathrm{o}}$ is the initial volume of the waste form, $\pi r_{o}{ }^{2} h$, and $A(t)$ is the area which can be expressed as: 


$$
L_{0} A(t) / V_{0}=2(1-u t)
$$

Using these expressions and placing this into the integral for $\mathrm{Q}(\mathrm{t})$ yields:

$$
Q_{c}=\frac{2 u M_{o}}{\lambda}\left[\left(1-\frac{u}{\lambda}\right)\left(1-e^{(-\lambda t)}\right)+u t e^{(-\lambda t)}\right]
$$

Evaluation of this expression at 273 years yields a cumulative fractional release of 0.38 , consistent with the prediction of the cylindrical finite-difference model, Table 5.12

For spherical geometry, $L_{0}$ is the initial radius $r_{0}, V_{o}$ is the initial volume of the waste form, $4 \pi r_{0}{ }^{3} / 3$ , and $\mathrm{A}(\mathrm{t})$ is the area which can be expressed as:

$$
\mathrm{A}(\mathrm{t})=4 \pi\left(\mathrm{r}_{\mathrm{o}}-\mathrm{ur} \mathrm{r}_{\mathrm{o}} \mathrm{t}\right)^{2}
$$

Using these expressions,

$$
\begin{aligned}
& \mathrm{L}_{\mathrm{o}} \mathrm{A}(\mathrm{t}) / \mathrm{V}_{\mathrm{o}}= 3(1-\mathrm{ut})^{2} \\
& Q_{c}=\frac{3 u M_{o}}{\lambda}\left[\left(1-\frac{2 u}{\lambda}+\frac{2 u^{2}}{\lambda^{2}}\right)-e^{(-\lambda t)}\left[(1-u t)^{2}-\frac{2 u}{\lambda}(1-u t)+\frac{2 u^{2}}{\lambda^{2}}\right]\right]
\end{aligned}
$$

Placing this into the integral for $\mathrm{Q}(\mathrm{t})$ yields:

Evaluation of this expression at 273 years yields a cumulative fractional release of 0.51 , consistent with the prediction of the DUST-MS spherical finite-difference model. Table 5.12. 


\subsection{DUSTINMS: A PREPROCESSOR THAT CREATES AN INPUT DECK}

\section{FOR DUST-MS}

In order to facilitate ease of use of the DUST-MS computer code, a pre-processor which takes the user through all of the steps necessary to create an input deck has been written. This preprocessor, DUSTINMS, relieves the code user of knowing the exact format and structure of an input deck and is menu driven. The menus present a series of choices and generally request a numeric response. In the few cases when an alpha-numeric response is required, e.g., defining titles, this is clearly noted. The DUSTINMS pre-processor was written before DUSTMS-D and does not allow access to all the features of DUST-MSD. Additions to the DUST-MS input file include allowing definition of the burial time for each container, the container failure mode (uniform and guassian) and the use of boundary condition files for the upstream mass flux boundary. These changes are discussed in Chapter 7, where the structure of the DUST-MSD input file is given.

DUSTINMS has the flexibility to independently alter any single parameter required by the DUST-MS code. DUSTINMS has the capability of creating an entirely new input deck or reading a partially or fully completed input deck which can then be modified. A major advantage in using the DUSTINMS code is that it provides an annotated input file for DUST-MS. This greatly facilitates direct modification of the input deck, Chapter 7.

The first menu asks the user if a completely new input deck is to be created or if an existing input deck is to be modified. After this decision has been made, the code proceeds to the main menu. The main menu permits the user to modify any variable required for input to the DUST-MS code independently through access to sub-menus. After the input variables have been defined, DUSTINMS allows the user to create a trial input deck or create a "partial" input deck. The flexibility allowed in being able to independently modify any single variable within the code makes it nearly impossible to guarantee that a consistent input deck is created by the DUSTINMS user. Although, there are many checks within the code to prevent obvious problems, it is possible to create an invalid input deck. For example, the user could define the number of containers to be 20 and not specify any failure parameters for the containers. If this occurs, DUSTINMS will try to make an input deck if requested, but obviously the input deck will not be valid. For this reason, DUSTINMS permits the user to create an output file that is identical to the output file that would be obtained if the DUST-MS code were used. This is a useful aid in debugging the trial input deck.

In addition, DUSTINMS permits the user to create a "partial" input deck which can be read in later by the DUSTINMS code and modified as necessary. Use of this feature is strongly recommended. A "partial" input deck is any set of input data created by DUSTINMS using the namelist procedure described later in this section. The advantage to this approach is that the check to determine if the data forms a valid input file is not made when reading the data. In contrast, if the user attempts to read in a completed input deck that is not valid, an error message is printed and 
control is returned to the operating system. That is, data on an invalid completed input deck cannot be modified by DUSTINMS.

The remainder of this chapter takes the reader through the various menus that appear when running the DUSTINMS computer code. All sections that have a double border and are highlighted in boldface print are the screens that appear on the console. Italic characters are examples of the code users response to the query. For convenience in referencing and to enhance the ease of locating discussions of the parameters, the numbering system for this chapter will correlate to the numbers in the main and secondary menus. For example, Section 6.4 .3 will refer to the fourth item on the main menu and the third item on the secondary menu. For this reason, the input selections menu and the main menu will appear in this section.

This chapter will serve as the most detailed reference on the input variables required for DUST-MS. In addition to describing the operation of the DUSTINMS code, selection of the appropriate values for the physical parameters, instructions on the operations of the models, and recommendations on when to use certain models will be primarily provided in this chapter.

\section{INPUT SELECTIONS:}

When running the DUSTINMS code, the first menu is:

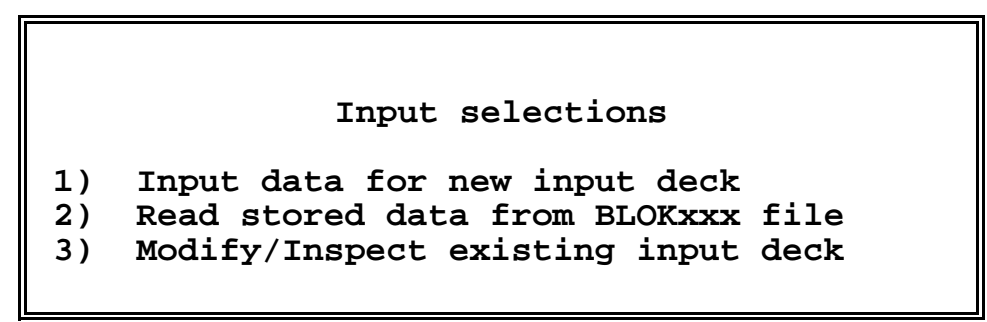

Choice $=1$ :

The code user will create an entirely new input deck. Default values are specified for some parameters but generally these need to be redefined. After choosing this value, the main menu is displayed.

Choice $=2$ :

Upon entering a value of 2 , the following screen is displayed:

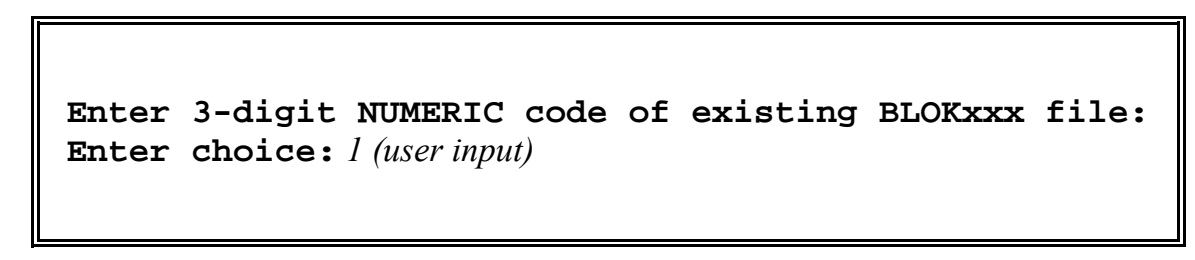




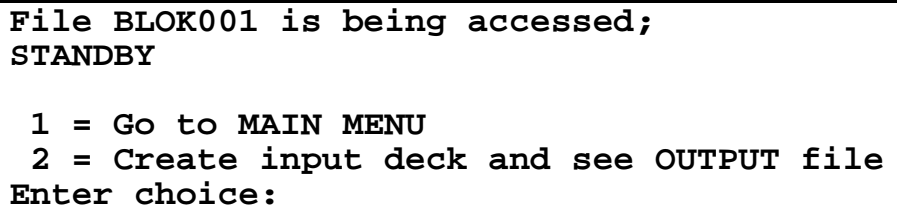

DUSTINMS will read a file previously prepared by DUSTINMS called BLOKxxx, where xxx is a three digit number. In the example, the 1 digit number is translated by the code to 001 and the file BLOK001 is read. BLOKxxx is a file created by using FORTRAN namelists. As such, it is an exact copy of all the input variables needed by the DUST-MS code at the time that the file was written. There are no checks on whether this set of variables forms a valid input deck. Therefore, the BLOKxxx file is useful when the code user is uncertain if a valid input deck has been created or if a partial input deck has been created.

The code checks to determine if the BLOKxxx file exists. If it does not exist, the user is returned to the Input Selections Menu. After successfully reading the file, the code user is allowed to attempt to make an input deck or proceed to the main menu.

Choice $=3$ :

The code requests the name of the input file, a name for the output file to be created, and if the output file exists, the code asks if the file should be overwritten. The completed screen for this procedure is displayed:

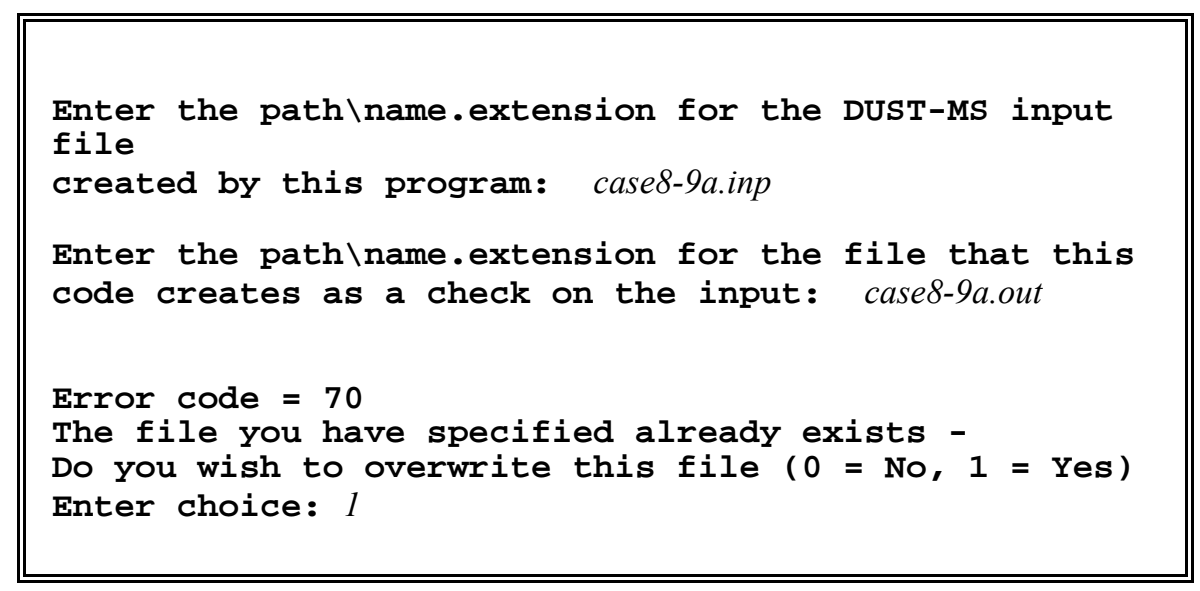

The DUSTINMS code then proceeds to the Main Menu. If the file case8-9a.inp did not exist or if the file does not contain a valid input deck, the code fails and control is returned to the operating system. 


\section{MAIN MENU:}

The main menu consists of a list of general categories that comprise the groups of input parameters needed by the DUST-MS code. The main menu provides access to the sub-menus defined by the grouping scheme. Definition of the input variables occurs in the sub-menus. This section will provide a description of the main menu and the variables contained under the grouping scheme. Detailed discussions on the variables and how they impact on model predictions will be presented when discussing the sub-menus. The main menu for DUSTINMS follows:

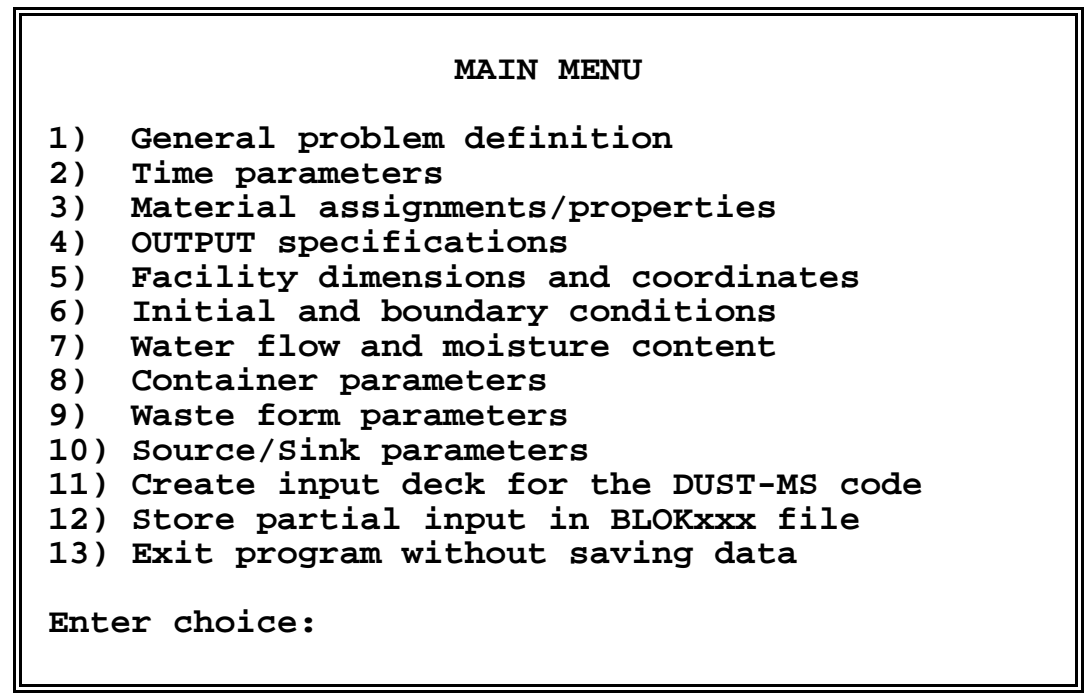

Choice $=1$ : General problem definition

The general problem definition includes variables which define the following: title, number of isotopes, a flag to specify if the input is in mass units of grams or curies (if the input is in curies, the code internally translates this to grams for consistency with the units on the distribution coefficient and solubility limits, upon output, the mass is translated back into the original input units), the number of control volumes in the computation, the number of decay chains, and the definition of the decay chains (length of the chain, members in the chain, and the fraction of decays leading to each progeny). In addition, for each isotope being simulated, the name of each radionuclide, half-life, atomic mass, and solubility limit are covered in this input deck.

Choice $=2:$ Time parameters

Time parameters include the number of time steps, the number of time step changes, the initial time step, fractional change in time step, maximum time step, and maximum problem time .

Choice $=3:$ Material assignments/properties 
The DUST-MS model allows multiple material types and requires the number of different materials. For each material type and isotope, the bulk density, soil distribution, dispersion, and diffusion coefficients is required. If there is more than one material, specification of the location of each material is required.

Choice $=4:$ OUTPUT specifications

Output specifications include printer control variables which determine if output occurs at each time step, the number and location of concentration and flux traces, and the number of time steps between writing values to the trace files.

Choice $=5:$ Facility dimensions and coordinates

The DUST-MS transport model allows variable thickness cells and, therefore, the thickness of each cell must be specified along with the facility surface area. The height of the facility is calculated from the input. The surface area of the facility is a normalizing factor to account for the volume of the 3-D facility in a 1-D model. It is an important parameter in determining concentrations of radionuclides in solution. For example, if the mass inventory is released instantly and uniformly across the facility, the concentration is determined from the inventory, height, surface area, and moisture content. Since dose is proportional to concentration, the normalization by surface area is required.

Choice $=6:$ Initial and boundary conditions

Initial and boundary conditions are required for each isotope being simulated. The DUST-MS code allows specified concentration, total flux, advective flux, or dispersive flux as a boundary condition.

Choice $=7$ : Water flow and moisture content

The Darcy velocity and moisture content are required. A spatially uniform, time-varying Darcy velocity, and a spatially varying, time invariant moisture content are modeled through tabular input. A time varying Darcy velocity could be used to model degradation in the ability of the cap to prevent water ingress.

Choice $=8:$ Container parameters

Container parameters include the number of containers, their location in the modeled domain and their failure time. In addition, DUST-MS allows localized (pitting) failure and, therefore, requires information on the localized failure rate parameters as well as assignment of these parameters to each container.

Choice $=9$ : Waste form parameters 
Waste form release parameters include the inventory, the number of different sets of release rate parameters, a flag to indicate which release rate parameters are to be used on each waste form, and the release rate parameters. The DUST-MS code has three release models: only rinse release subject to partitioning, an uniform fractional release rate corrected for radioactive decay and diffusion controlled release from either cylindrical or rectangular finite sized waste forms. Therefore, input is required to define the diffusion coefficients and the dimensions of the waste form. Release rate parameters are defined for each isotope in the simulation.

Choice $=10:$ Source/Sink parameters

In addition to the waste forms releasing contaminants into the system, for each isotope, external sources or sinks can be specified by the code user. These sources are defined through tabular input which provides the source strength as a function of time, as well as input specifying the number of sources, the number of different types of sources (e.g., number of source strength versus time tables), the location of the sources, and the assignment of a source type to a location.

Choice $=11:$ Create input deck for the DUST-MS code

This selection creates the input deck to be used by the computer code DUST-MS. It first asks for the file name for the input file. If it exists, it asks the user if it should overwrite this file. It then asks if an attempt to create an output file should be made. If so, it requests the name of the output file. After completing this task, control is returned to the Main Menu. This can be useful when creating multiple decks in which only a few parameters change.

Choice $=12:$ Store partial input in BLOKxxx file

This option writes the file BLOKxxx where the values for $\mathrm{xxx}$ are determined through input. After completing this task, control is returned to the Main Menu.

Choice $=13:$ Exit program without saving data

Selection of this value exits the program. Control is returned to the operating system. 


\subsection{General Problem Definitions}

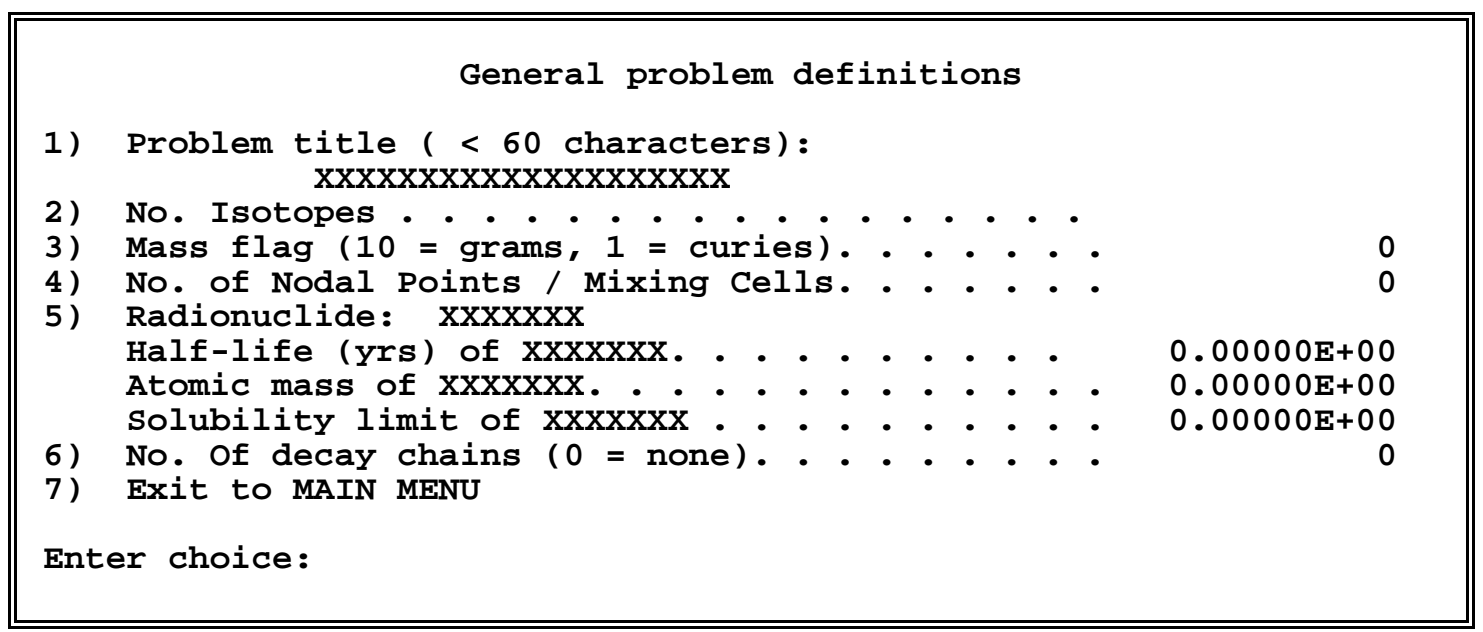

On every sub-menu the most recent values for the various parameters are printed when the menu is accessed. The XXXX values for alpha-numeric characters indicate input has not been specified. In this example, the user is creating a new deck and nothing has been specified. If the user had read in an existing input file, the values would be those found in that file. If more than one nuclide is specified, the first nuclide is listed under item 5. Aces to set the properties of other nuclides can be obtained by inputting selection 5. Similarly, access to define decay chain information is obtained by selecting choice 6 .

\subsubsection{Problem Title}

An alpha-numeric string up to 60 characters in length that describes the problem. Use of the character "/" causes problems when creating BLOKxxx files because the namelist procedure takes this as a variable delimiter. Therefore, use of "/" is not recommended.

\subsubsection{Number of isotopes}

DUST-MS can simulate up to 10 species in a single run. This number defines the number of species.

\subsubsection{Radionuclide}


DUSTINMS has an auxiliary file called RNUCL2.DAT that contains over 200 radionuclides, their half-life in years, atomic mass, and a default solubility limit of $10 \mathrm{gm} / \mathrm{cm}^{3}$. The atomic mass is used when converting mass between curies and grams. The solubility limit is used in controlling release. It is well known that solubility values are highly dependent on the environment. The default value has been selected to be large enough that solubility limits will not influence release. If better values for this number are defensible, they can be input to the code. When choice 2 is selected, the following menu is displayed:

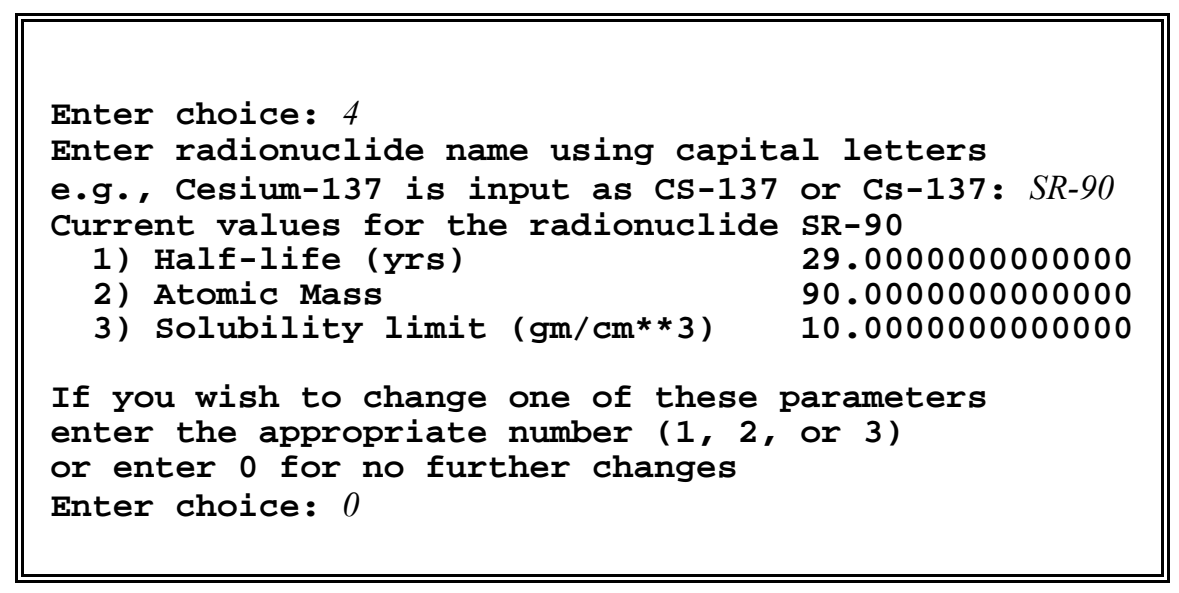

Capital letters are required for the radionuclide name because the computer code takes the input name and performs a string comparison with the radionuclides on the file RNUCL2.DAT. If the radionuclide name does not match any of those on file, the code allows the user to either try another name, or enter the values for half-life, atomic mass and solubility limit.

\subsubsection{Units Flag for Mass Input}

Mass can be input in units of becguerels, curies or grams. If the input is in becguerels or curies, the code uses the half-life and atomic mass to convert to units of grams. This is done for consistency with $\mathrm{Kd}$ values, waste form partition coefficients, and solubility limits. For user convenience all output is converted back to the original input units. That is, if the input is in curies, the output is in curies.

\subsubsection{Number of Computational Cells}

The number of nodal points are input. Currently, the maximum value is dimensioned at 500 .
6.1.6
Decay Chains 
DUST-MS can simulate up to 10 decay chains and up to 10 members in a decay chain. This number provides the number of decay chains to be modeled. If this number is greater than 0 , another menu is presented and the user defines the length of the chain, identifies the radionuclides in the chain, and specifies the fraction of decays that lead to the progeny. In most cases, the decay fraction is 1.0 (i.e, $100 \%$ of the decays of the parent lead to the progeny). In some cases, when a radionuclide can undergo more than one decay path, this fractional decay will be less than one. For example, if a radionuclide decayed to progeny X $75 \%$ of the time and progeny $\mathrm{Y} 25 \%$ of the time, the fractional decay would be 0.75 in the first decay chain, and 0.25 in the second decay chain.

\subsection{Time Parameters}

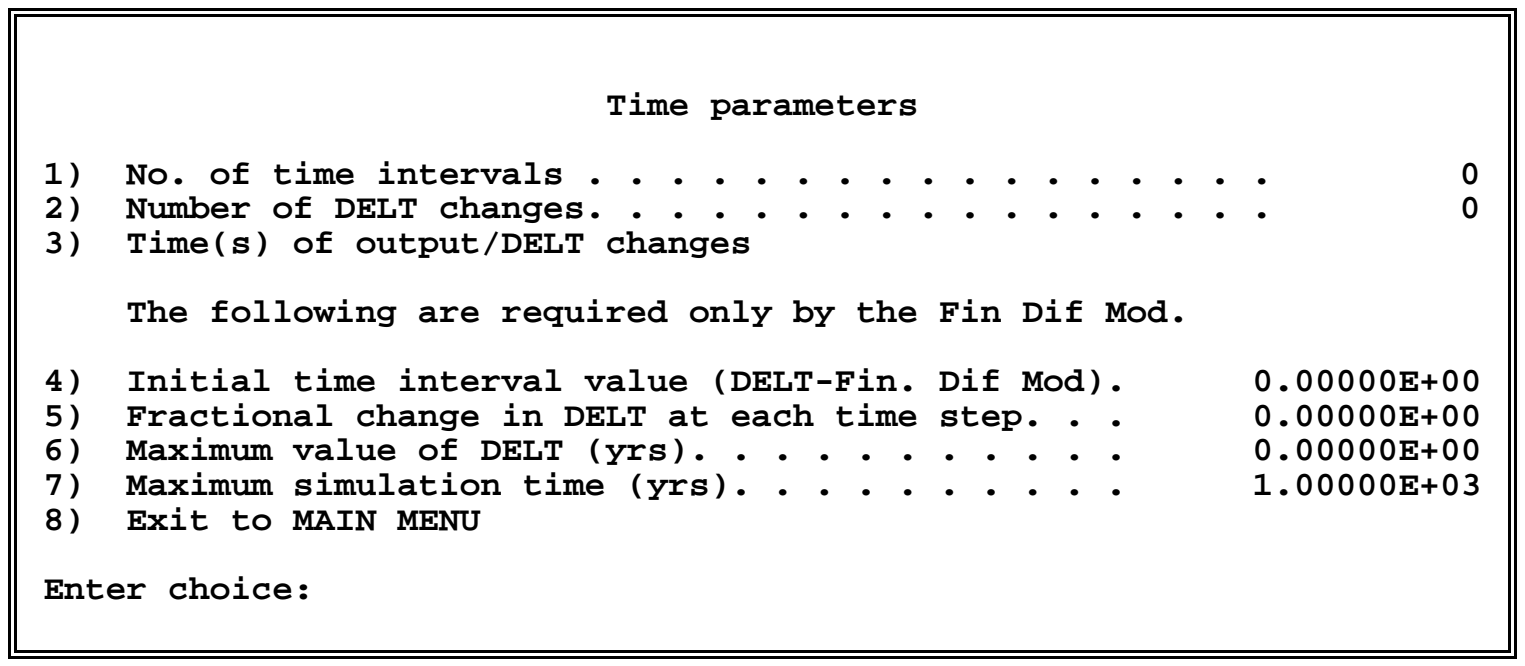

\subsubsection{Time Intervals}

The maximum number of time steps permitted in solution of the finite difference transport equations. There is no maximum value for the number of time intervals.

\subsubsection{Number of Time Step Changes}

The time step size is determined from the initial time step and a multiplier applied at the end of each time step. The size of the time step is limited by a maximum value of the time step, (DELMAX). For example, if the time step is 1 , and the fractional multiplier is 0.1 . The second time step is 1.1 , the third is 1.21 , etc. The increase is applied until the maximum value is reached, Sections 6.2.4 - 6.2.7. Time step logic within the code permits the time step to be reset to the initial time step at user specified times. This can be useful if it is known a priori that an event that will 
change the movement of contaminants will occur at a specific time. For example, if it is known that containers fail at 20 and 50 years, the user may want to have relatively small time steps around the times of container failure. This can be achieved through resetting the time step at these two times.

\subsubsection{Output Times or Time Step Changes}

In the sub-menu, it should be noted that a value is not listed for this parameter. This occurs because this parameter requires an array of values. Upon selection of this sub-menu item, the current values in the array are printed. All parameters that require an array are presented in this fashion.

This input specifies the time at which the time step is to be reset to its initial value. If this value is greater than the maximum problem time, the time step is never reset.

\subsubsection{Initial Time Step}

Initial time step size in years.

\subsubsection{Fractional Time Step}

The fractional change in time step is input in this section. Assuming that the maximum time step has not been reached and the time step has not been reset, the time step size at the N-th calculation is:

$$
\mathrm{DT}_{\mathrm{N}}=\mathrm{DT}_{\mathrm{i}} *(1+\mathrm{FRX})^{(\mathrm{N}-1)}
$$

whereDT $_{\mathrm{N}}=$ the $\mathrm{N}$-th time step.

$\mathrm{DT}_{\mathrm{i}} \quad$ initial time step, and

$\mathrm{FRX}=$ fractional change in time step.

At the time at which the time step is reset to its initial value, the value of $\mathrm{N}$ is reset to 0 .

\subsubsection{Maximum Time Step}

Maximum value for the time step (years). This value should be selected based on the problem being modeled and the degree of accuracy required in the solution.

Although the solution procedure used is fully implicit, as a rule of thumb, the contaminant should not move more than the width of a computational cell in one time step. Therefore, the following relationship should be maintained: 


$$
\frac{V_{D} \Delta t}{R \Delta x}<1.0
$$

where $\mathrm{V}_{\mathrm{D}}$ is the Darcy Velocity, $\Delta \mathrm{t}$ is the time step size, $\mathrm{R}$ is the retardation coefficient and $\Delta \mathrm{x}$ is the cell width. The above expression can be used to obtain a maximum time step.

\subsubsection{Maximum Simulation Time}

Maximum problem time (years), the default value is 1000 years.

\subsection{Material Assignments/Properties}

Up to 10 different material types can be specified throughout the facility. This additional flexibility requires that each control volume is assigned a material type. The code automatically defines all control volumes as material type 1 . Therefore, definition of material type is required only for control volumes that are not material type 1. The material properties menu is:

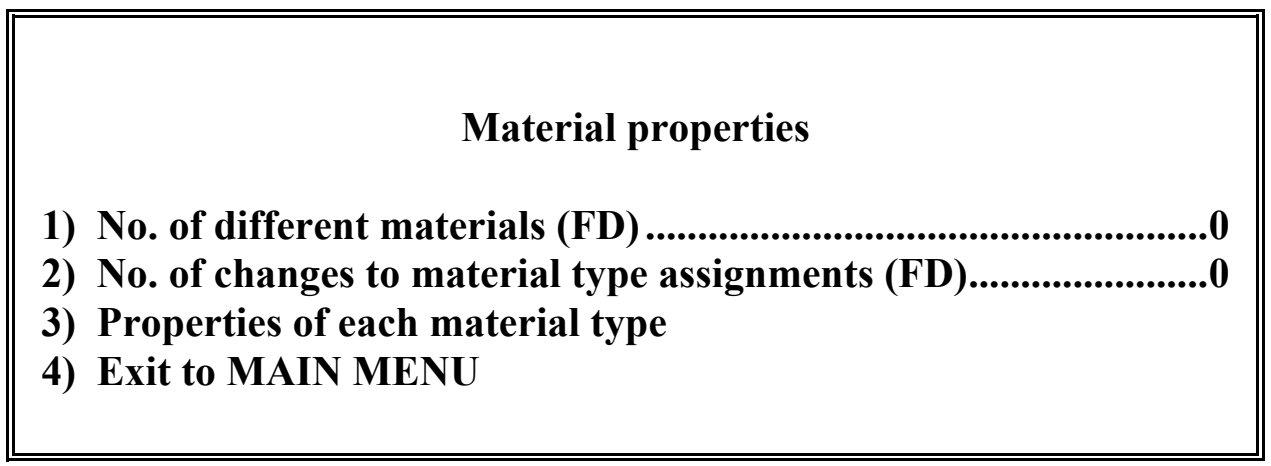

\subsubsection{Number of Different Materials}

The variable specifies the number of different materials in the facility. The maximum value is 10.

\subsubsection{Material Type Assignments}

Material type reassignment is achieved through the following routine. Explicit instructions and variable definitions are provided when running DUSTINMS as in the example that follows. 
In this example, the code asks for the number of material type reassignments. To minimize input errors, the DUSTINMS code uses a routine that requires the user to input values within a specified range. The maximum and minimum of the range are determined internally by the code and are based on consistency with previously defined values. In this example, the maximum value of 100 is determined as the maximum number of control volumes as specified in Menu 1.4.

In this case, the code user asked to reassign 10 nodes (control volumes). The code then prints the instructions and definitions for the input variables. The first of which is the node number for the first node in this sequence. Again, Min and Max values are calculated by the code. The user selected a value of 90 .

The code asks for the number of nodes to be reassigned in this sequence. The user requested 10 , the total number previously specified. If the first node in the sequence was 95, the code would permit only 6 nodes (nodes 95 - 100 inclusive) to be redefined. For the increment between nodes, the code calculated that the maximum increment was 1 and forces the code user to input a value of 1 . If for example, the user asked to redefine 2 nodes starting at node 90 , the maximum increment would be 10 (i.e., nodes 90 and 100 could be defined on this card) and any value between 1 and 10 would be acceptable.

The code asks for the material type to assign to the first node in this sequence. The maximum value of 3 was determined from the value specified in Menu 3.1. The code user selected material type 3.

The increment in material type to each node was determined to be zero (there are only 3 materials). In a 1-D simulation most often, the materials will occur in layers covering several nodes and zero will be the appropriate choice. However, if the material types change in a regular fashion, this feature may be used.

Upon completing this sequence of input, the code prints out the values and asks if they are acceptable. In the example, we have defined nodes 90 - 99 to be material type 3 . Note, node number 100 is still material type 1.

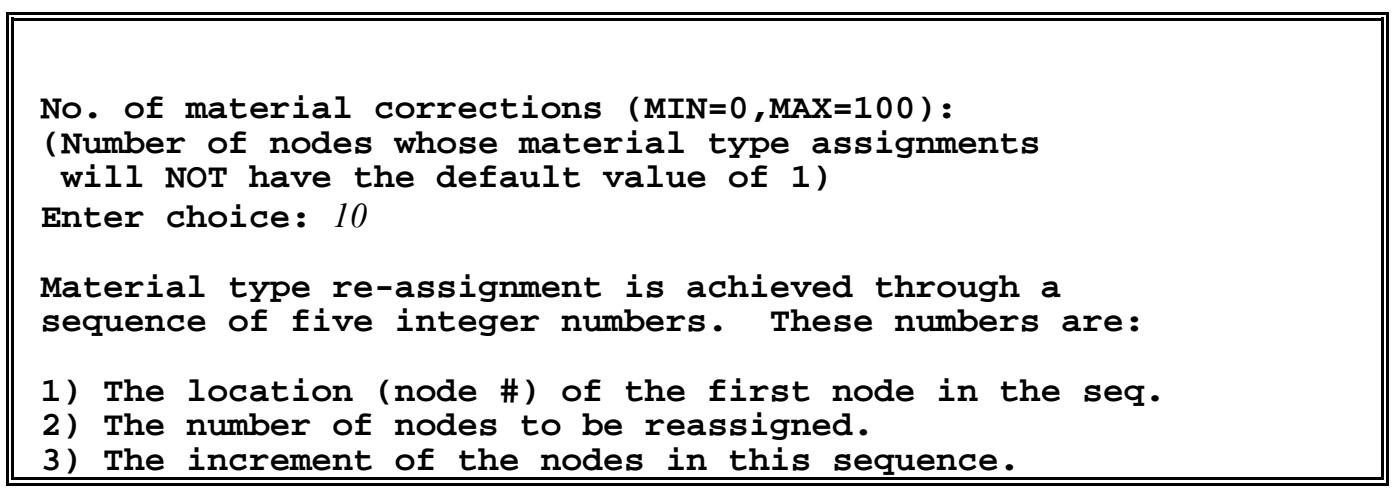




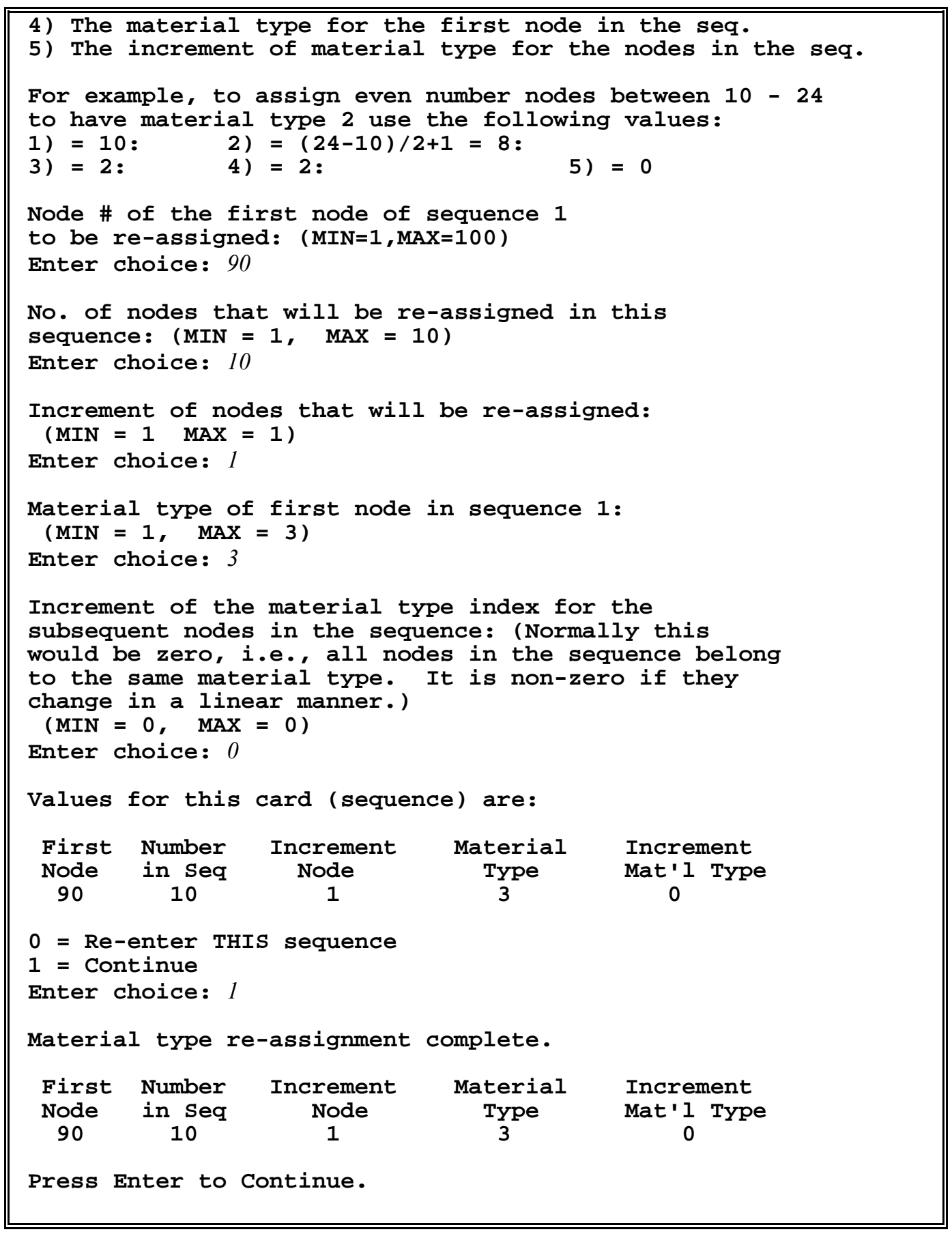

The code checks to determine if the number of material type reassignments equals the total number specified in Menu 3.1, if it does, the sequence of numbers that define material type are printed. If it does not, the code returns to the beginning of this section and asks for more reassignments. Although there is a check on the total number of reassignments, there is no check on whether a node has been reassigned twice. For example, the user could specify that nodes $1-5$ are 
material type 2 in the first sequence and then specify nodes $3-7$ are material type 3 in the second sequence. The code would then only define 7 nodes having material properties different than material 1.

\subsubsection{Material Properties}

Four soil material properties (distribution coefficient, bulk density, dispersivity, and diffusion coefficient) are required. The following example provides a typical input session for the first material. First, the code prints out the existing values for all material types (specified in Menu 3.1). If the user decides to change some of these properties, the code asks for the material type to change, prints out the existing values for that material and allows the user to change any one of the values independently or all of the values simultaneously. In this example, all of the values are changed. The units required for the various parameters are printed to remind the user that cgs units are used for material properties. After the user has input the parameters, the code prints these values and allows the user another chance to change the input. If the input is correct, the code returns to the materials properties menu and asks for changes in other materials. 
Material properties:

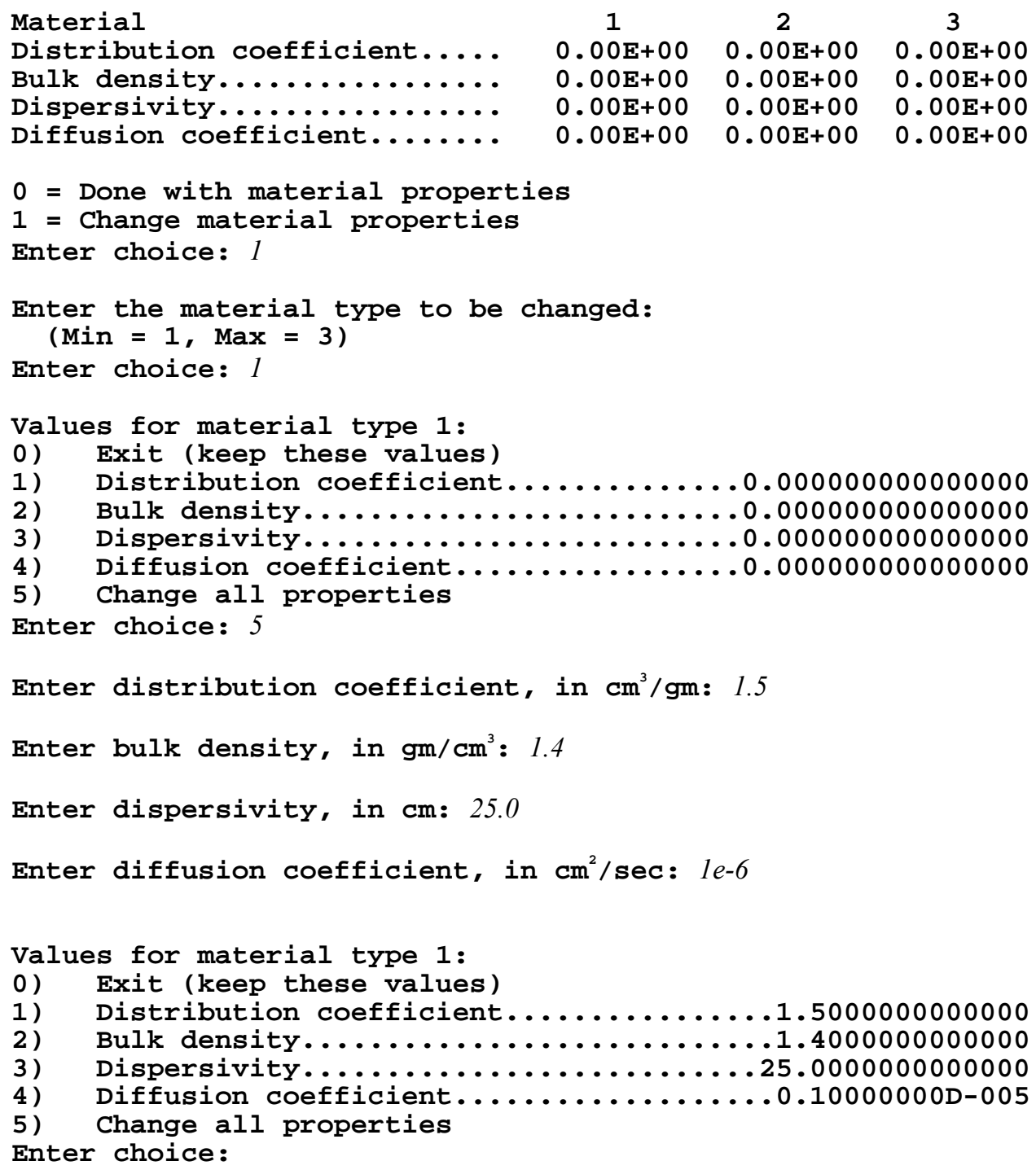

\subsection{Output Specifications}

This sub-menu controls output produced by the DUST-MS code.

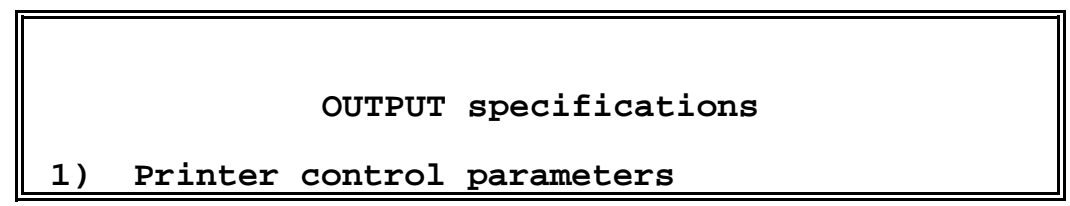




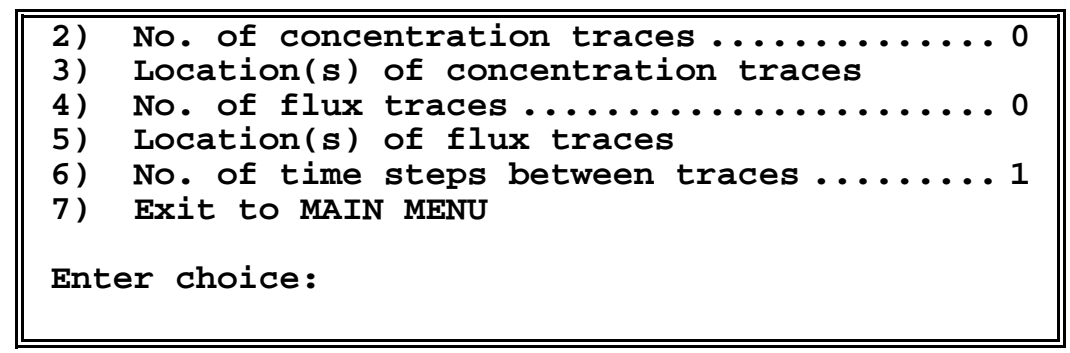

\subsection{1}

\section{Printer Control Parameters}

The first menu item controls the main print out. In DUST-MS there is a variable KPR(I) where I ranges from 1 to the number of time steps.

If $\mathrm{KPR}(\mathrm{I})=0$, nothing is printed at the I-th time step. If $\mathrm{KPR}(\mathrm{I})=1$, the concentration at each finite difference point is printed. If $\mathrm{KPR}(\mathrm{I})=2$, the concentration and flux are printed. If $\mathrm{KPR}(\mathrm{I})=$ 3 , container breach and waste form release parameters are printed. If $K P R(I)=4$ and the finite difference leaching model is specified, the concentrations withing the waste form are printed. When using DUST-MS, often many intermediate calculations are performed to enhance numerical accuracy, therefore, zero is the default value for all items in KPR. Thus, in order to obtain the main output, some of the values for KPR must be specified. At every time step in which KPR $>0$, the concentrations at every location are written to the file CONCNT.DAT. This file can be used by the program GRAFMS to graph the data on the video console.

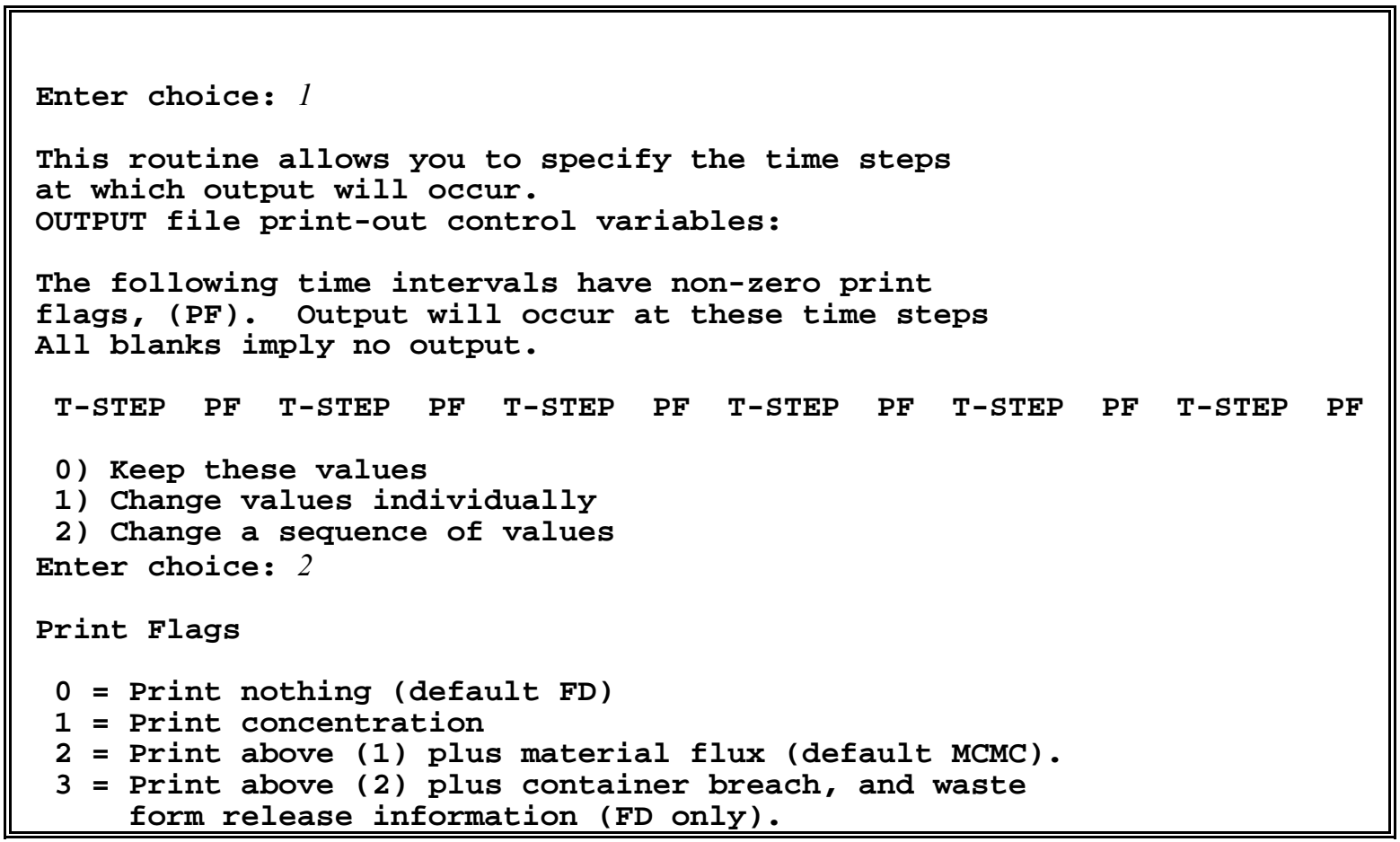




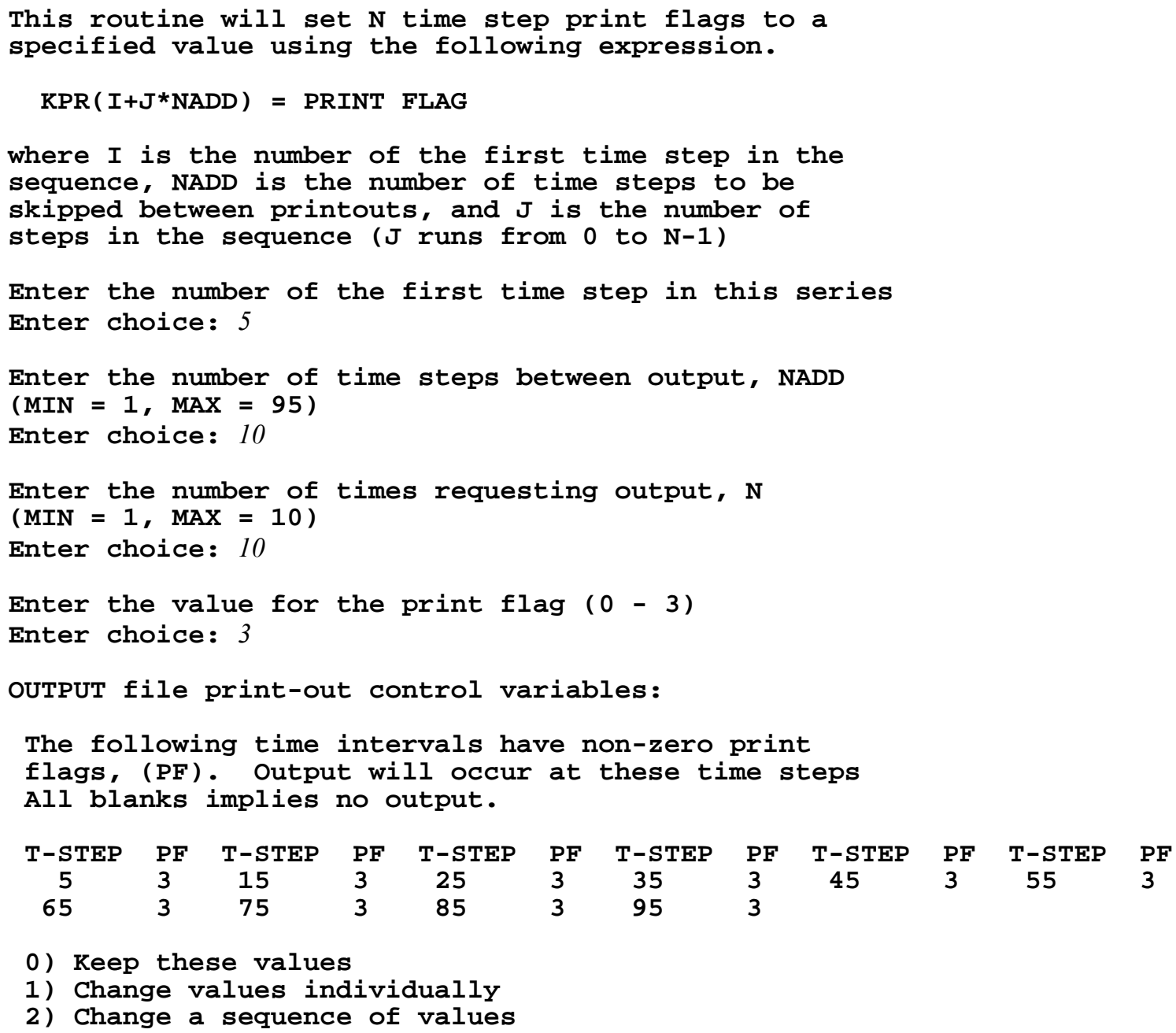

0) Keep these values

1) Change values individually

2) Change a sequence of values

Enter choice: 0

In the preceding example, the initial values for KPR are zero. The user selects to obtain the full printout, $K P R=3$, starting at time step 5 and for every 10-th time step after this. Instructions on how to input this information are provided while running the code as shown. After specification is complete, the code returns to the output specifications menu.

If option 1, change values individually, were selected the code asks for the number of values that are to be changed and then uses this number to repeat a sequence of questions asking for the time step and print flag to be specified. Again, instructions are provided during operation of DUSTINMS.

\subsubsection{Number of Concentration Traces}

Requesting a concentration trace requires the DUST-MS computer code to write a file containing concentrations at a specified location as a function of time. Up to twenty locations can be specified. The output file created by DUST-MS is called TRACCND?.DAT. The ? Takes on a 
value of 0 - 9 to represent the various isotopes in the simulating. TRACNCD!.DAT contains isotope 1 information, TRACCND2.DAt contains isotope 2 information and so on. If 10 isotopes are simulated, the maximum number permitted, TRACCND0.DAT contains the information for that isotope. These files can be used directly by the graphics program GRAFMS.EXE and a plot of concentration versus time can be viewed immediately after running the DUST-MS code. Similar remarks apply to the TRACEFXD?.DAT files which contain information on the flux, mass flow rate, and cumulative mass that has passed by the measurement point.

\subsubsection{Location of Concentration Traces}

The location of the concentration traces are determined by specifying the node (or mixing cell) at which a trace should be made. In DUSTINMS the user is given the option of redefining a single trace node, or all of the trace nodes. In the following example, trace nodes had been previously specified and the user decided to change the last trace node from 50 to 60. After this, the code would print out the new values for trace nodes and ask if any further changes were desired.

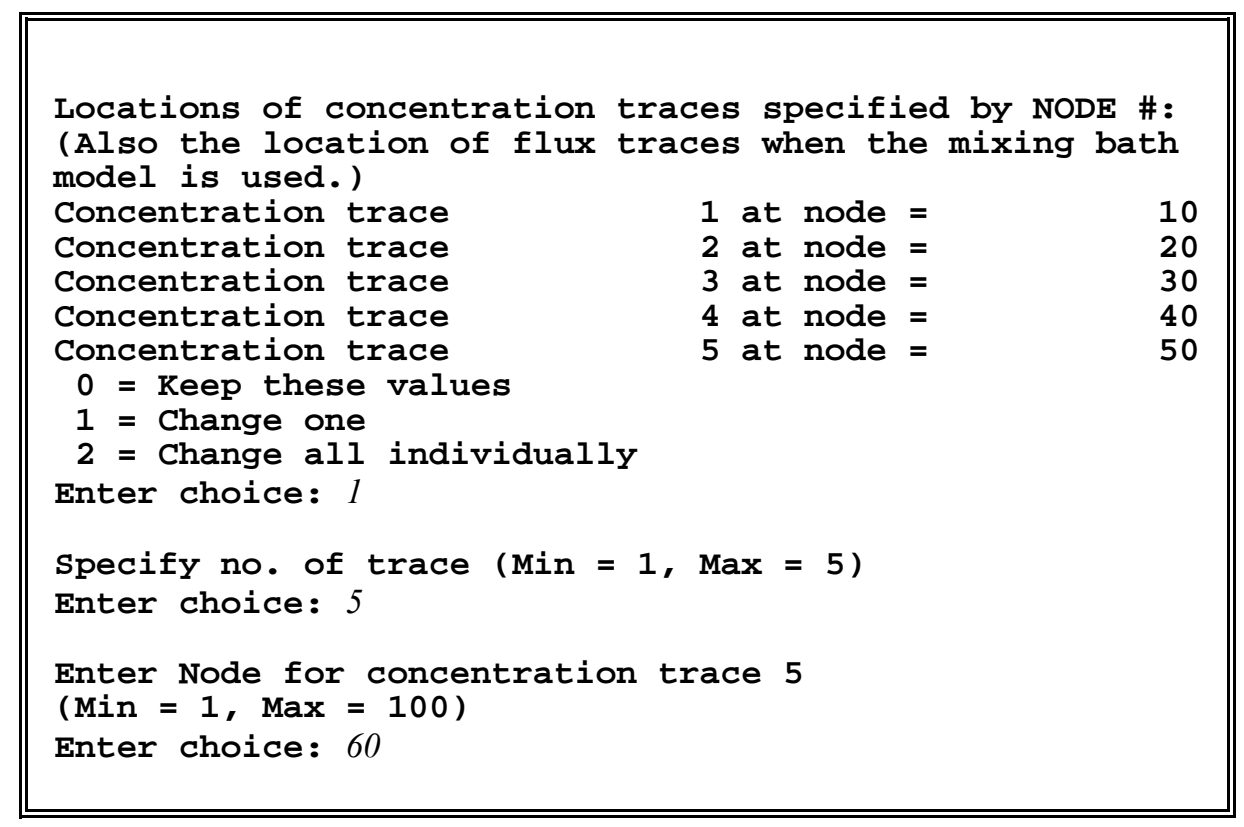

\subsubsection{Number of Flux Traces}

Requesting a flux trace requires DUST-MS to create a file containing the instantaneous mass flux, total mass (flux integrated over time multiplied by the surface area of the facility (Menu 5.2)) that has passed through that point, and mass release rate (flux multiplied by the surface area). The total mass is calculated numerically by summing the product of the flux, facility area, and time step. 


\subsubsection{Location of Flux Traces}

The location of flux traces are determined by specifying the node (or mixing cell) at which a trace is requested.. The input procedure for specifying location for flux traces is identical to that of concentration traces.

\subsubsection{Number of Time Steps between Traces}

This value specifies how frequently the trace files are updated. If the default value of 1 is used, traces are written at every time step. If a value of $\mathrm{N}$ is used, traces are written every $\mathrm{N}$-th time step.

\subsection{Facility Dimensions and Coordinates}

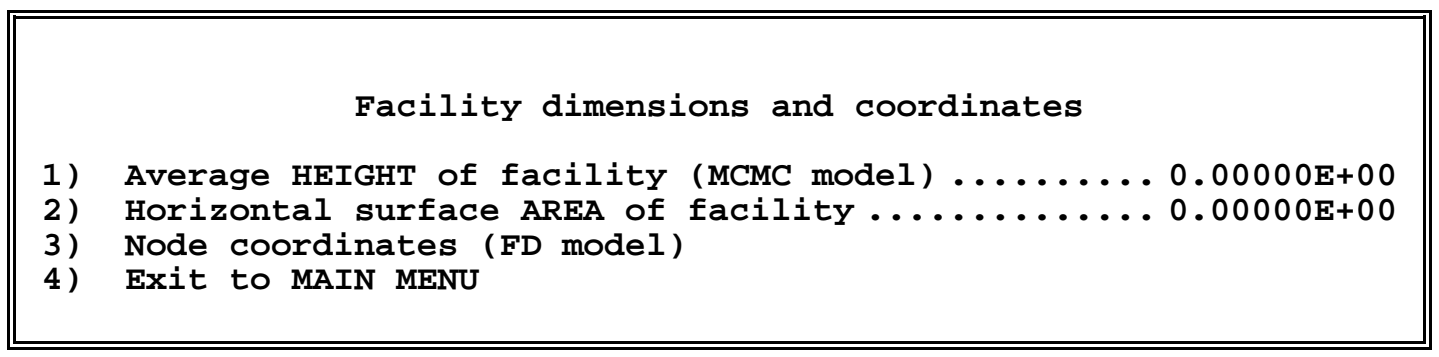

\subsubsection{Surface Area}

The surface area is a normalization factor that permits scaling of the concentrations (mass per unit volume) to account for the 3-D facility in a 1-D model. The volume of a computational cell is the height of the cell multiplied by the surface area. Obtaining the correct concentration is important as the dose to man scales linearly with concentration. The area must be input in units of square centimeters.

\subsubsection{Node Coordinates}

The DUST-MS model permits non-uniform mesh spacing. This is useful when modeling different regions which exhibit different transport characteristics. The values for the node coordinates represent depth and can be positive or negative. However, Node 1 is always the highest node (least deep) and the last node is the lowest node (greatest depth). When entering coordinate 
values, the coordinates must increase with depth and the user must input the coordinates in order, beginning with Node 1. (The DUSTINMS code forces the user to begin with Node 1 and continue sequentially to the last node.) The distance must be in units of $\mathrm{cm}$. For regularly spaced nodes, automatic generation of the nodes can be specified through the formula in the following example.

Upon entering this menu, the following description of the input requirements is presented.

Node coordinates
The values for the node coordinates represent DEPTH,
and can be positive or negative. Node 1 is always
highest (least deep), and the last node is always
lowest (greatest depth).
We recommend assigning node 1 a depth of 0.0 cm. In
any case, coordinates must INCREASE with increasing
node number.
Press Enter to Continue.
Input of the nodal coordinates is accomplished by
using a series of cards that will automatically
calculate the coordinates using a regular sequence
In particular, this routine will ask for:
a) the location of the first node in the sequence, (X) (I));
b) the distance between nodes in this sequence, DELTAX, and;
c) the fractional change in node size, XFACT.
d) the number of nodes in a sequence (N);
The location of each node is calculated from:
X(ItJ) = X(I) + DELTAX* (1+XFRAT) * J
where J=0 to N-1,

The example below defines nodes 1 - 50 having a mesh spacing of $10 \mathrm{~cm}$ beginning with node 1 at $x=0$.

Coordinate of node $1(\mathrm{~cm}): 0.0$
Distance between each node for the nodes in this
first sequence $(\mathrm{cm}): 10.0$
Fractional change of the distance between nodes over
the preceding distance: 0.0
No. of nodes that will be assigned coordinates in
this first sequence, including node 1




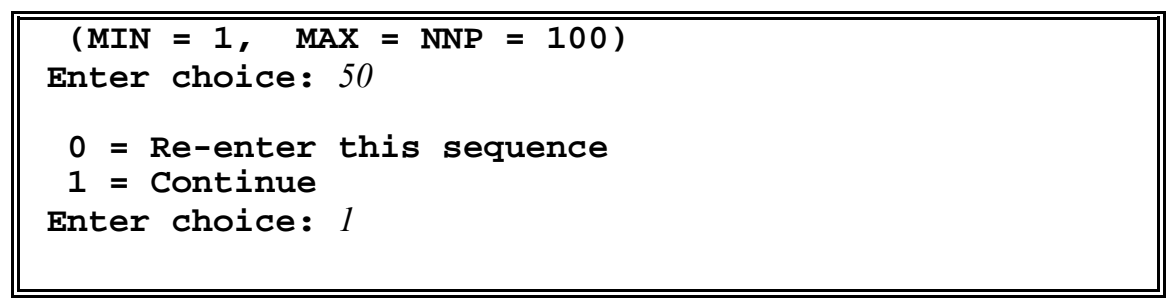

The DUSTINMS code permits the user to re-enter the sequence if an error occurred. If the sequence is correct, the code automatically checks to make sure that every node receives a coordinate value. In this case, more nodes need to be defined and the code calculates the value of the last node and the number of nodes remaining to be defined, and asks for more input. In this example, nodes $51-100$ have a mesh spacing of $20 \mathrm{~cm}$ beginning with $\mathrm{X}(51)=500 \mathrm{~cm}$.

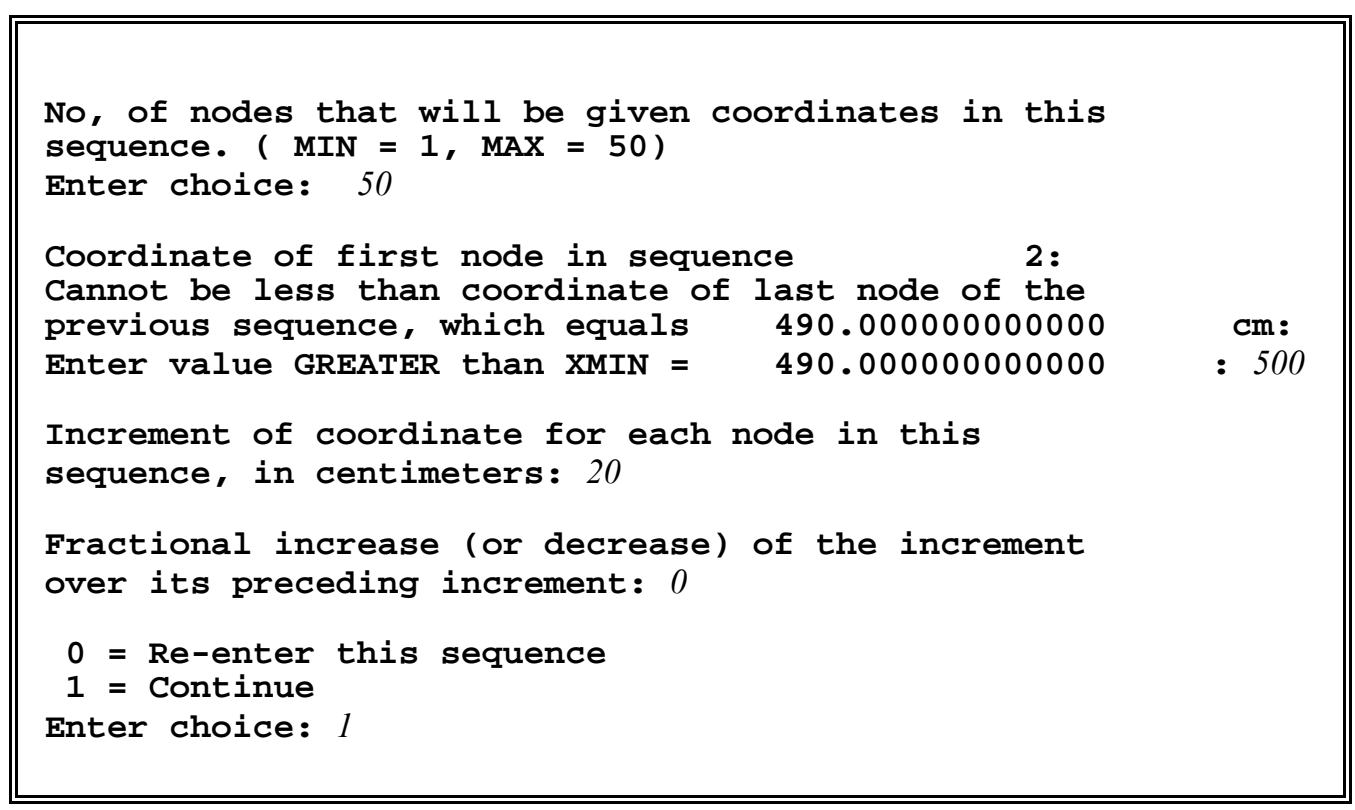

After completion of the input, the code prints the values requested in the form used by the DUST-MS code.

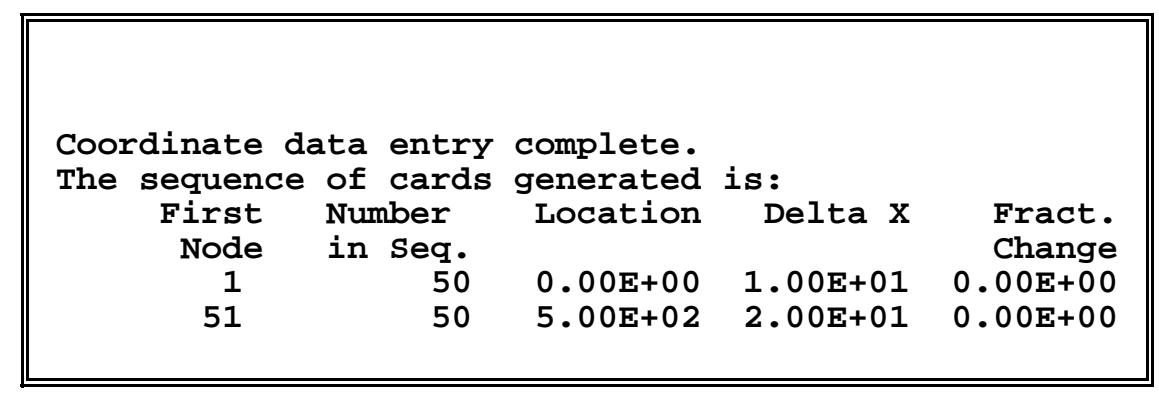




\subsection{Initial and Boundary Conditions}

The following menu appears for selecting initial and boundary conditions..

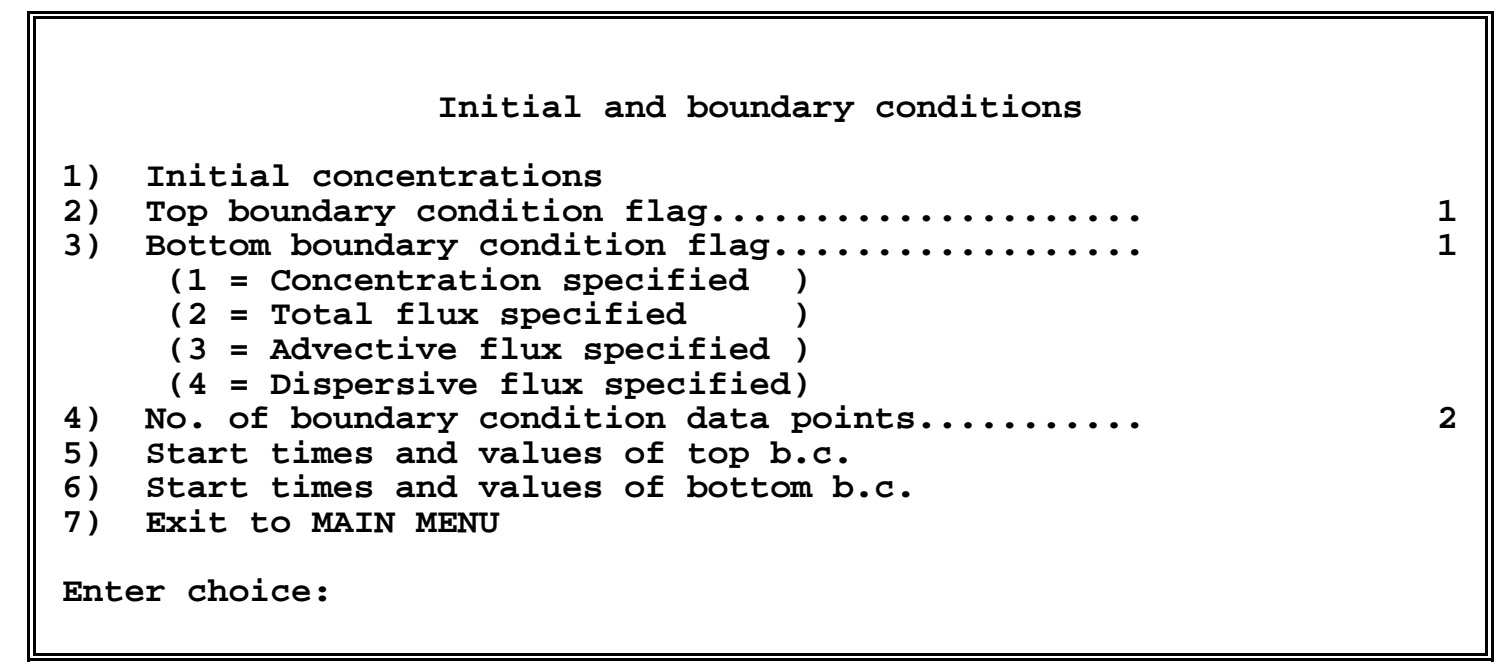

\subsubsection{Initial Conditions}

The initial concentration at every finite difference point must be specified. If a number of concentrations are equal or change in a regular manner, the input can be greatly simplified by entering a sequence of three numbers which represent the number of nodes (locations) at which the concentration is to be defined, the value for the concentration at the first of these nodes, and the amount to add to each successive node. DUSTINMS requires that the initial conditions be specified beginning with Node 1 continuing sequentially to the last nodal point. Upon completion of specifying all concentrations, the sequence of values are printed and control is returned to the Boundary and Initial Conditions submenu. If the initial condition is zero everywhere, the user can request the code to generate the appropriate input automatically.

The instructions printed by DUSTINMS when entering this menu are:

Initial condition: CONCENTRATIONS

0 = Use DEFAULT of 0.0 initial concentration at all locations. 


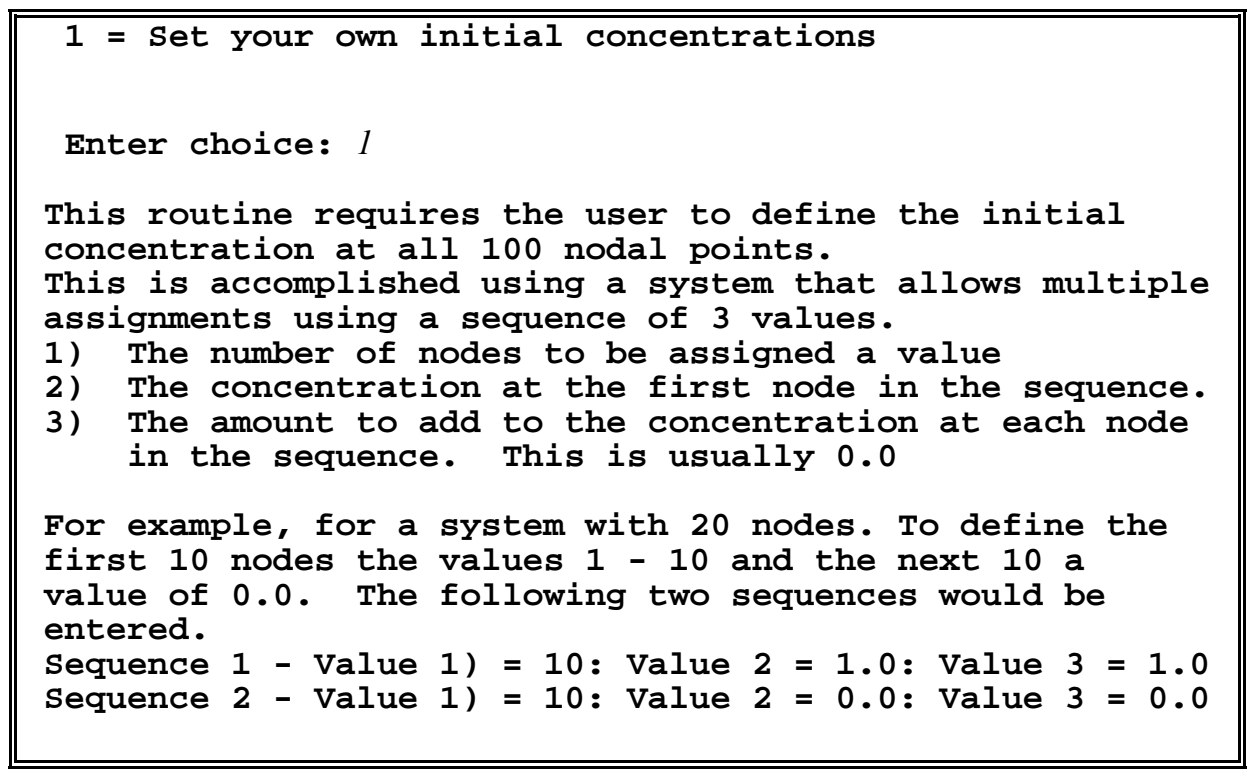

An example in which 100 nodal points have been requested follows. The first 50 nodes have an initial concentration of one. The units of concentration are either gms $/ \mathrm{cm}^{3}$ or Ci $/ \mathrm{cm}^{3}$ depending on whether the mass inventory is input in units of grams or curies (Menu 1.3 Mass Flag).

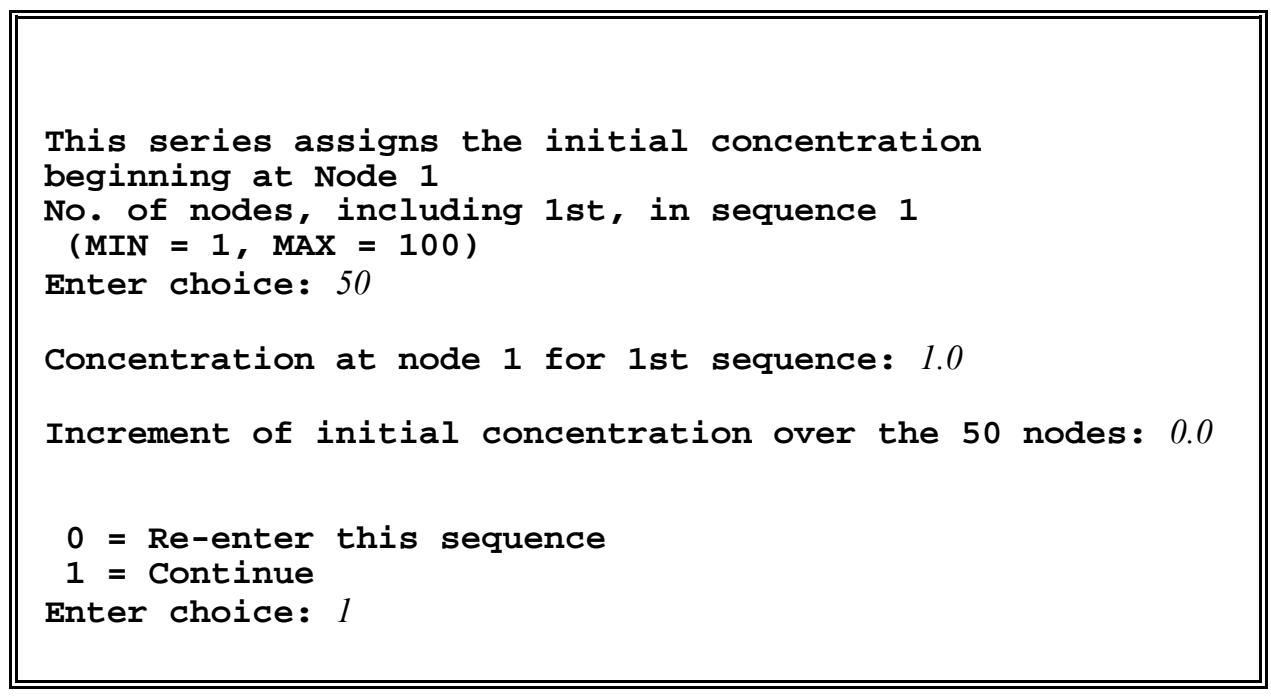

At this point, the code recognizes that not all 100 nodes have been given an initial concentration and more data is requested.

This series starts from Node 51

No. of nodes including 1 st, in sequence 2:

$(\mathrm{MIN}=1, \mathrm{MAX}=50)$ 


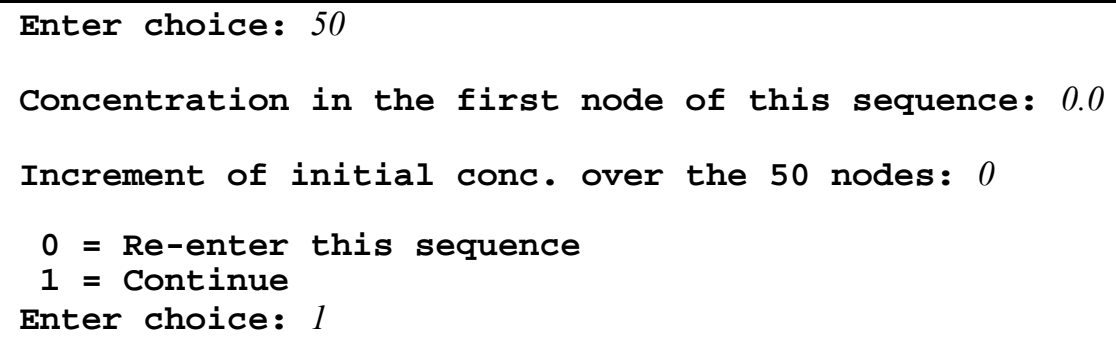

At this point, all nodal points have a specified initial condition and the code prints out the input values in the form required by the DUST-MS code.

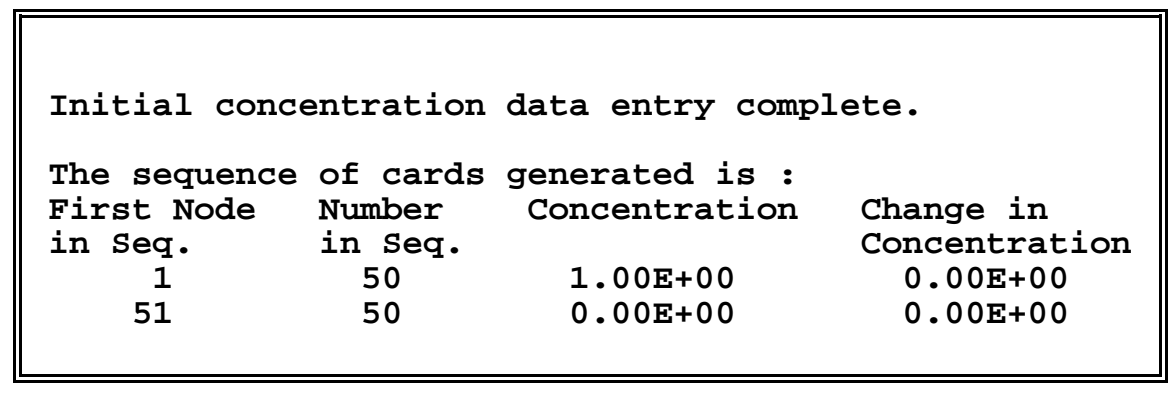

\subsubsection{Top Boundary Condition Flag}

Four choices for boundary conditions are allowed within the DUST-MS code. The concentration, total flux, advective flux, or diffusive/dispersive flux can all be specified as a function of time. These are described in detail in Section 2.6.

This input value selects the boundary condition from among these choices. The default value requires the concentration to be specified.

\subsubsection{Bottom Boundary Condition Flag}

The choices are identical to those at the top boundary as described in 6.6.2.

\subsubsection{Number of Boundary Condition Data Points}

All boundary conditions are specified as a function of time through a table containing the value of the boundary condition and the time in years. At any given simulation time, the value of the boundary condition is determined through interpolation of this table. The last time in the boundary 
condition table should be greater than or equal to the maximum simulation time. If this is not done, unpredictable results may occur. The minimum number of data points required to specify the interpolation table is 2 . The maximum number allowed by the code is 10 .

\subsubsection{Boundary Condition Table for the Top Boundary}

Boundary conditions (BC) are input as ordered pairs of time (years) and BC value (where the units are consistent with previous input, e.g., if mass is input in curies, mass units for the $\mathrm{BC}$ are in curies).

The following example has 4 data points in the table (specified in Menu 6.4), a maximum simulation time of 100 years (specified in Menu 2.7) and sets the concentration to 1.0 between 0 and 10 years and 0.0 after 10.01 years. Between 10 and 10.01 years the concentration varies linearly between 1 and 0 . Upon entering this submenu, the code prints out the existing values for the BC (all zero by default in the example). After a decision has been made to change the values, the code prompts the user for the first concentration value and forces the first time in the table to 0.0 years. After this, the code prompts the user for the time and boundary value. These are input on a single line separated by a space. After all of the boundary points are specified, the table is printed for review by the user.

\subsubsection{Boundary Condition Files}

DUST-MS permits the use of an auxiliary input file to provide boundary conditions of specified mass flux. This is useful for simulation of the transfer of mass from the unsaturated zone to the aquifer which will have different flow and transport characteristics. This option is permited in the Windows pre-processor, DUSTWIN, but not in the DOS version of the pre-processor. These files are created by the code DUSTAQIN.EXE which reads tracfxd?.dat files created by DUST-MS. The boundary condition files are called BOUND?.DAT, where the ? is a numeral which identifies the isotope under simulation.

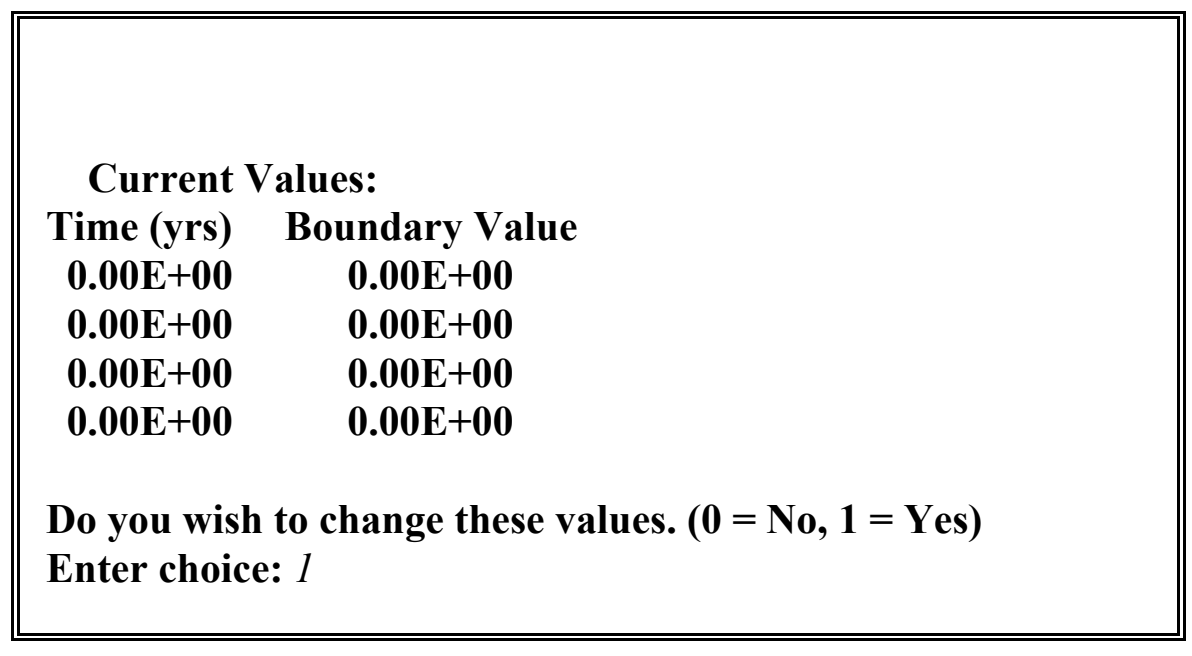




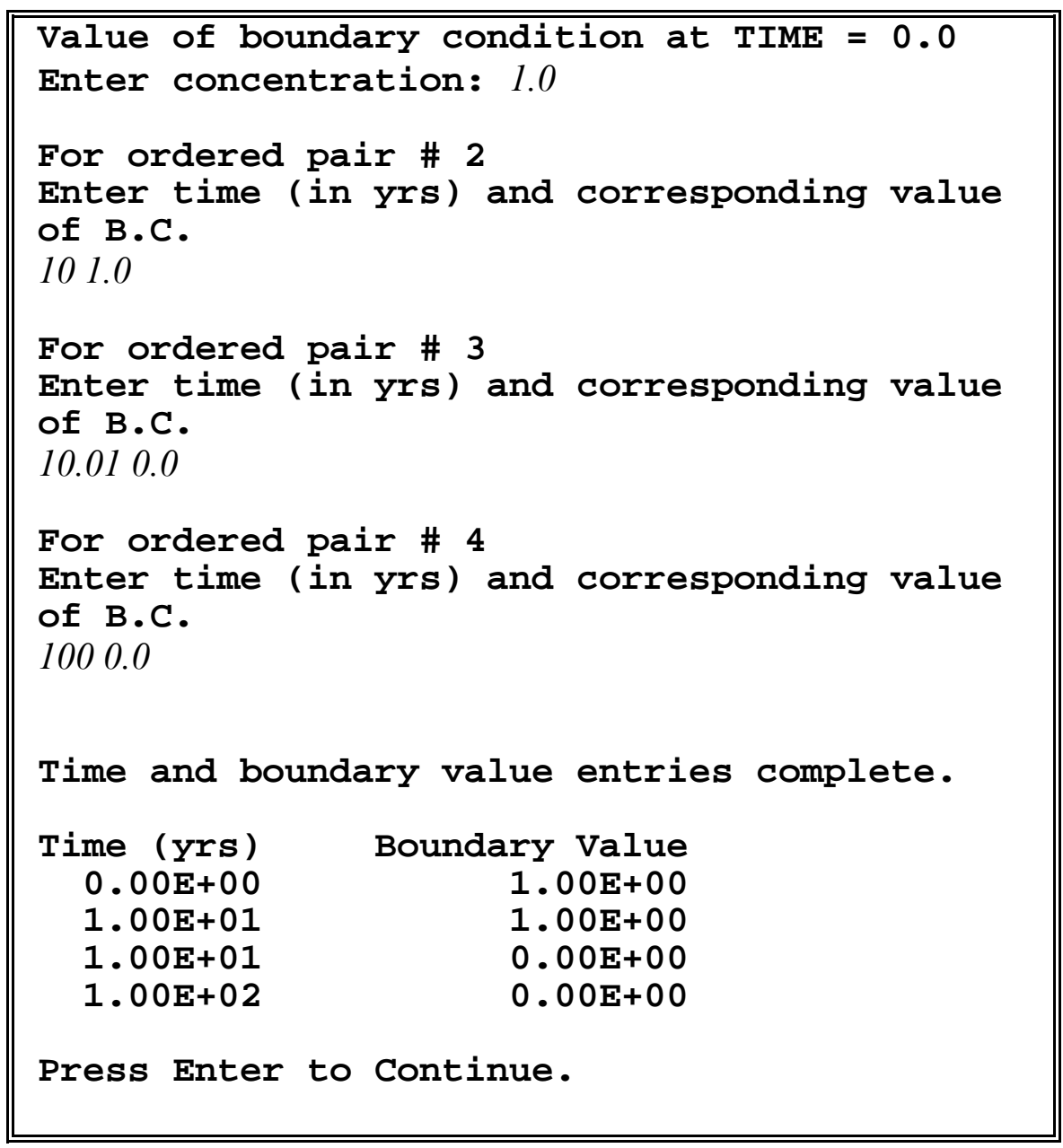

\subsubsection{Boundary Condition Table for the Bottom Boundary}

This procedure is identical to that of the top boundary. For details see Section 6.6.5.

\subsection{Water Flow and Moisture Content}

DUST-MS permits the Darcy velocity to be obtained as a function of time through tabular input in an approach similar to that of specifying the boundary conditions. The moisture content is permitted to vary spatially. The values for the Darcy velocity and moisture content should be obtained from numerical simulations with site specific data. The menu is:

Water flow and moisture content 


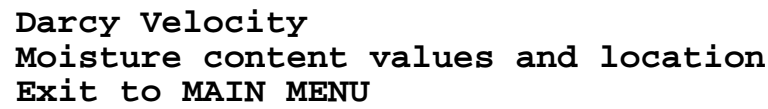

\subsubsection{Darcy Velocity}

A table of velocity versus time may be input or a single value for the Darcy velocity may be used. If a single value is used, the code asks the user for that value and creates the input table by specifying this value at $t=0$ and $t=T M A X$ (Menu 2.7). If a table is input, the velocity at any simulation time is calculated through linear interpolation using the table. If the last time value in the table is less than the maximum simulation time, unpredictable results may occur.

In the following example, the user has decided to specify an increase in velocity over time between 0 and 1000 years. DUSTINMS prints current values for the table ( 0 by default) and asks if a change is requested.

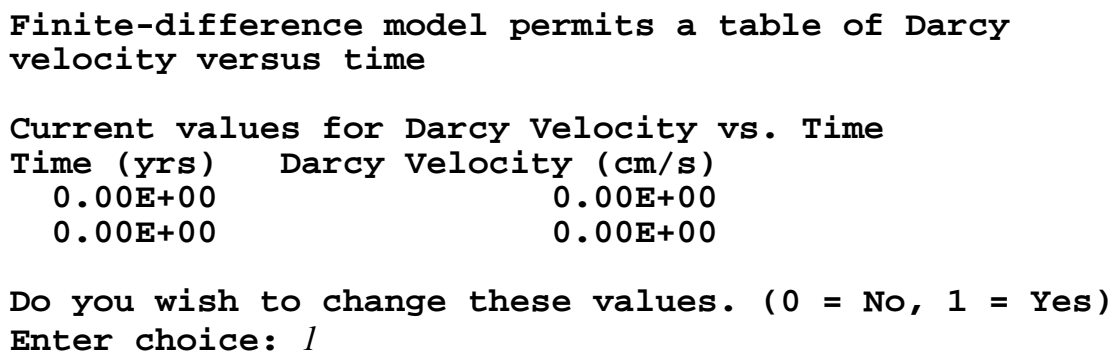

At this point, the user is provided a choice of using a single value or inputting a table of values. If a table of values is desired, the user inputs the number of points in the table and the code then prompts the user for the values. The code forces the first time value to be at zero years.

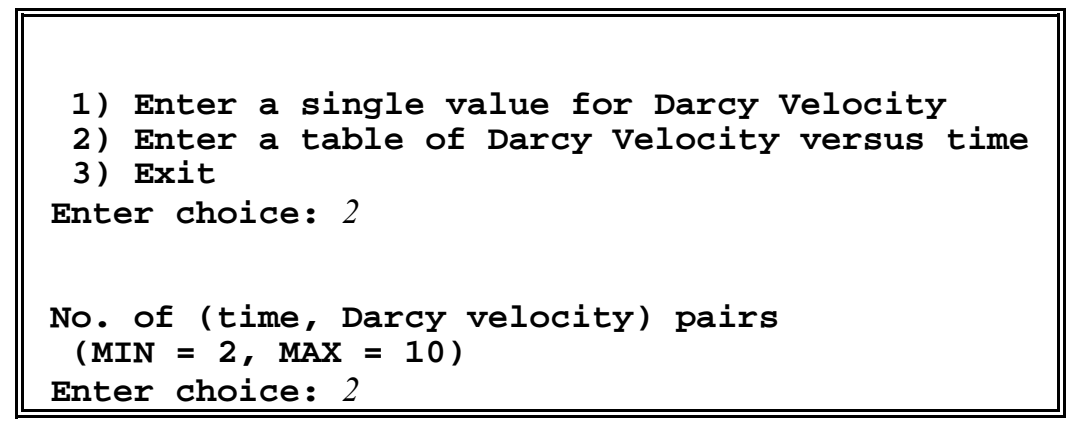




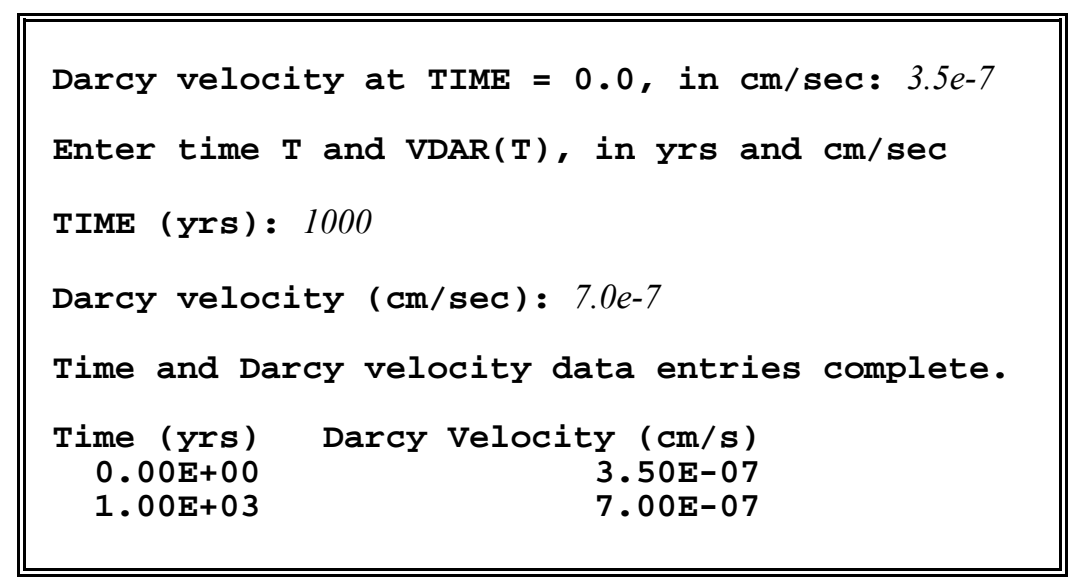

\subsubsection{Moisture Content Values}

The moisture content may vary with position. The values for moisture content should be determined from detailed simulations of the flow around the facility. In assigning moisture content values, the user must specify a value for every control volume. If the moisture content values remain constant or change linearly between control volumes for a region of the modeled domain, automatic generation of the moisture content can be prescribed.

The DUSTINMS code forces the user to enter the moisture content for each control volume (node), beginning with Node 1 and continuing sequentially until all nodes are specified. When entering this menu, existing values are printed in the form required as input by DUST-MS and the user is asked if a change is desired.

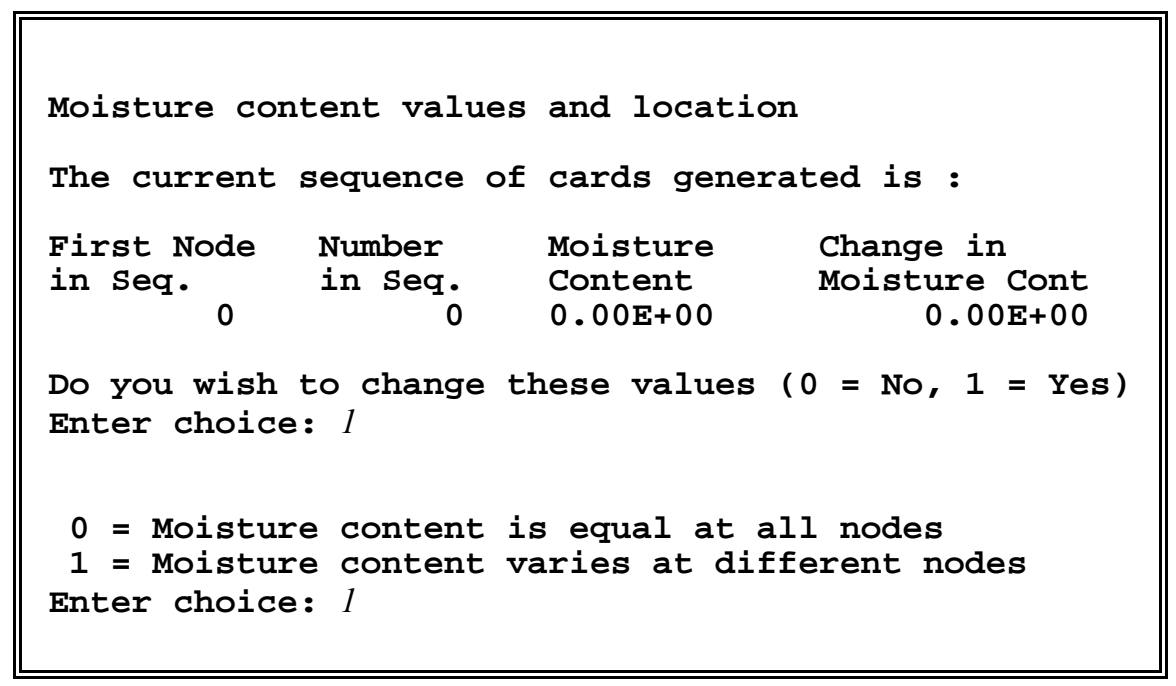


If the moisture content is constant ( 0 entered on the previous menu), the code asks for the value. If the moisture content changes with location, the following instructions are printed.

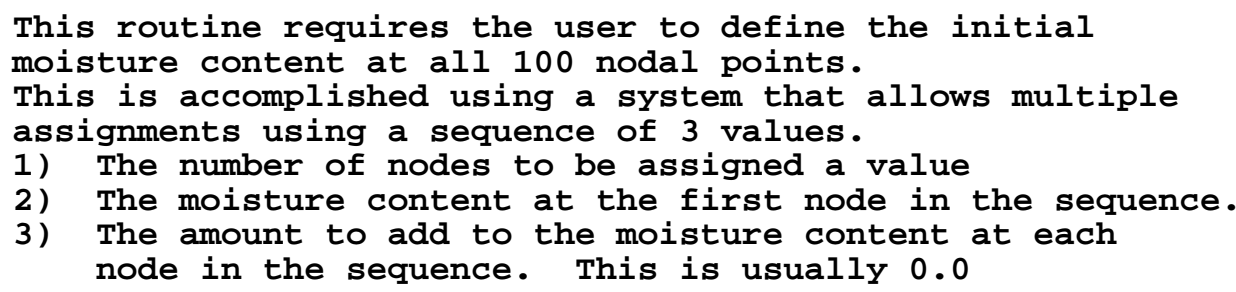

In the following example, the number of nodes has been specified to be 100 and the moisture content is set to 0.25 for the first 50 nodes and 0.3 for the last 50 nodes.

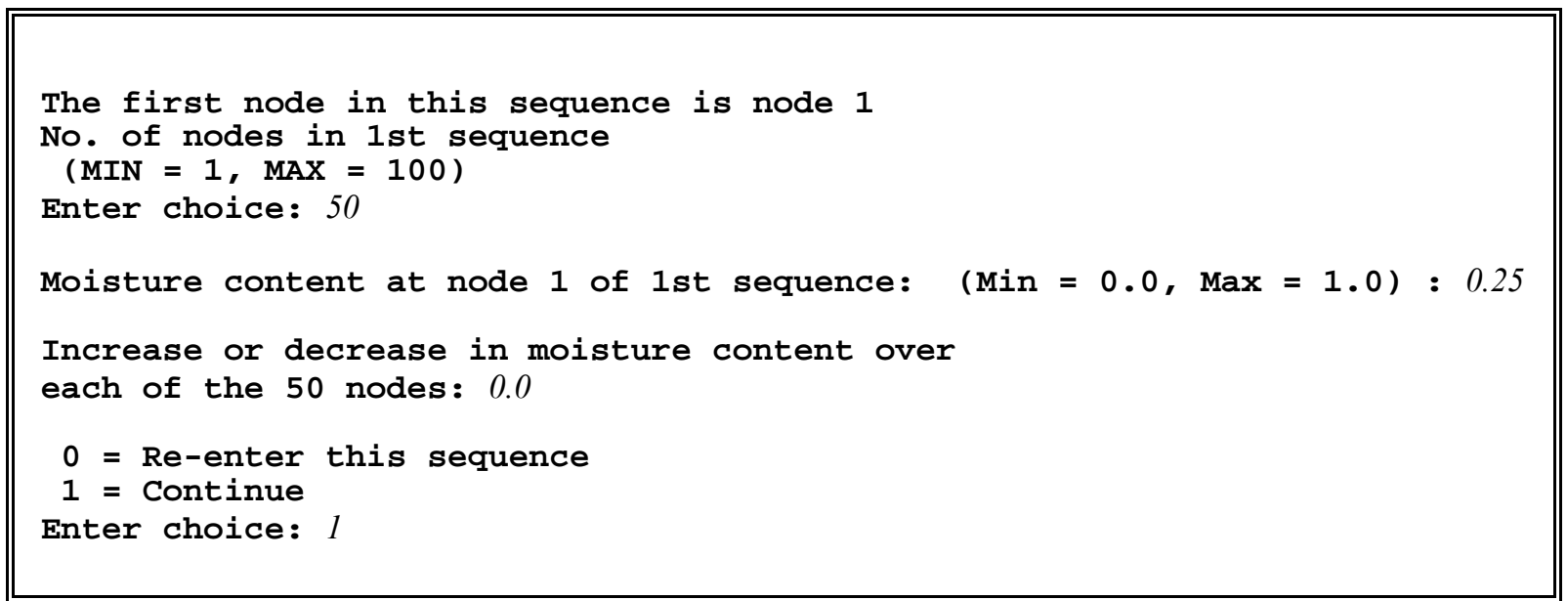

At this point, the code recognizes that only 50 of the 100 nodes have been given a value for moisture content. Therefore, it prints the instructions out and requests more input. Notice that the code begins this sequence at Node 51, the first undefined node.

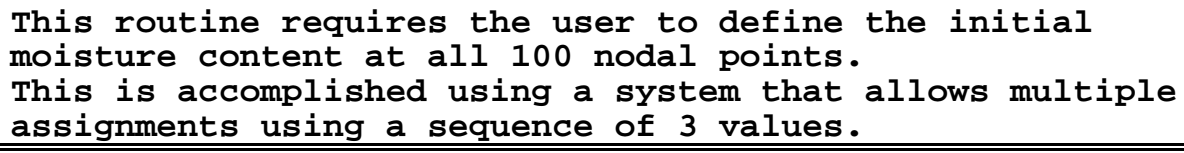




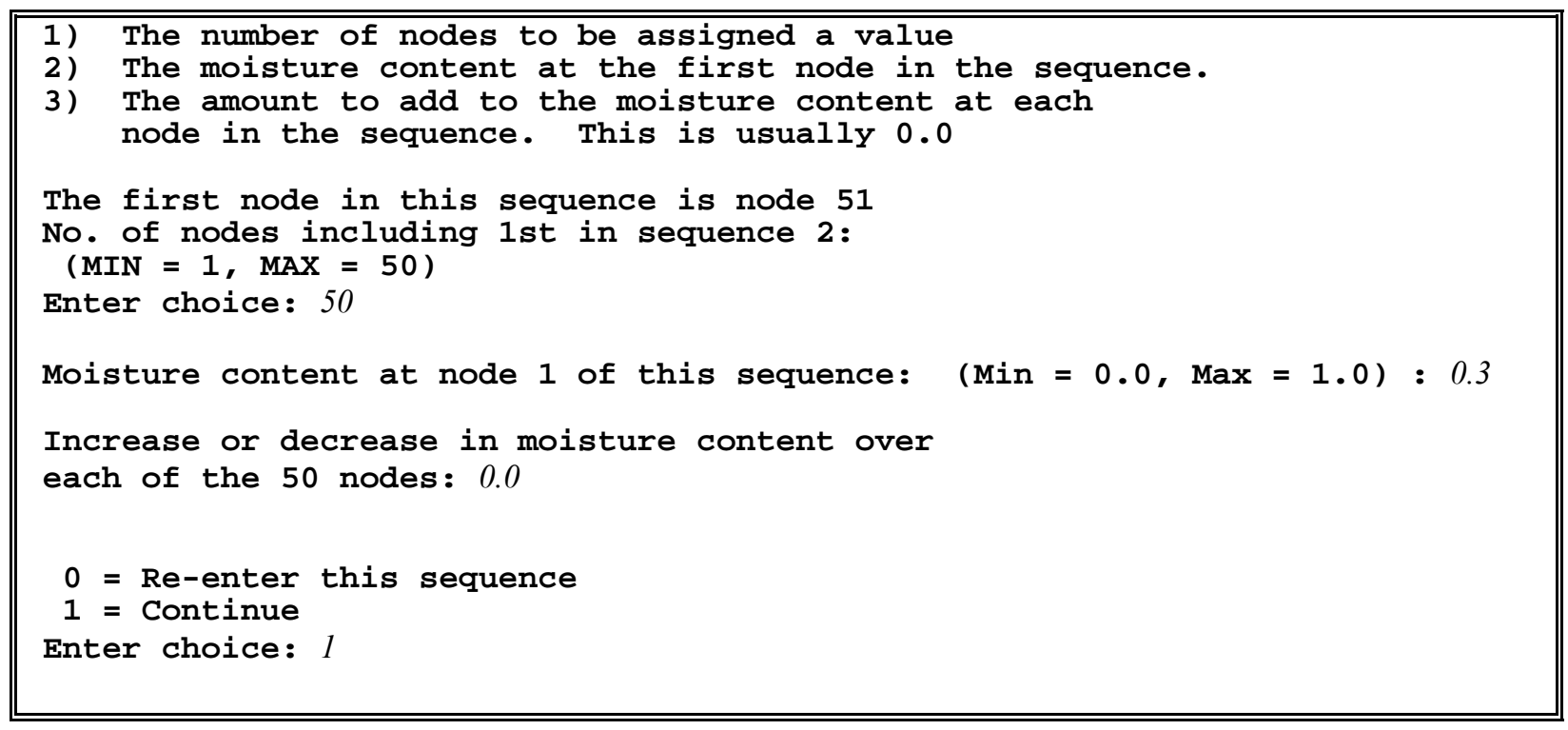

Upon specifying all moisture content values the code prints out the input cards needed by the DUST-MS code.

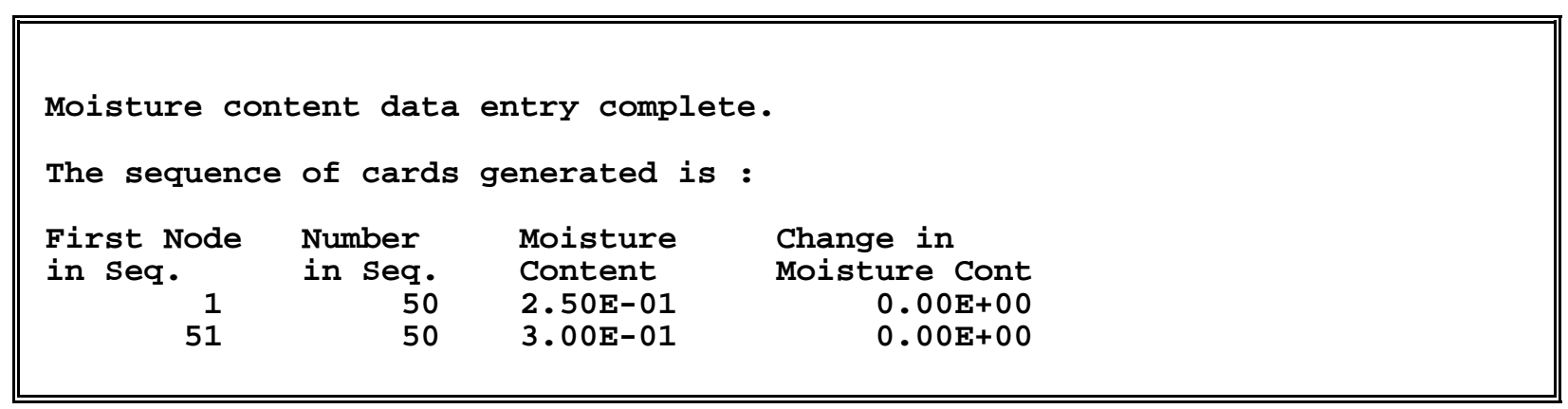

\subsection{Container Parameters}

The DUST-MS computer program allows up to 300 different containers each with a unique time to failure. The DUST-MS model permits up to 20 different localized container failure rates to be applied to the containers. Therefore, it requires input on the rate parameters, and integer flags that specify which set of rate parameters should be used for each container. The container menu is:

Container parameters

1) No. of containers .....................

2) Time of general failure for each container 


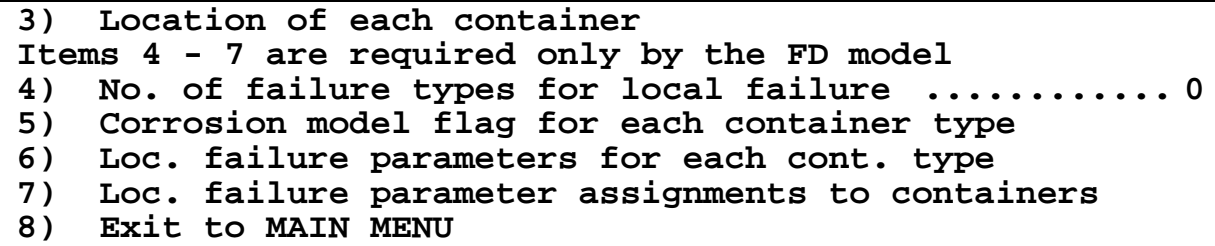

\subsection{1}

\section{Number of Containers}

This input variable provides the total number of mixing cells or control volumes that have a waste form source.

\subsubsection{Time of General Failure}

Each container has a time of general failure after which the container no longer provides a barrier to release from the waste form. These times should be estimated based on the material, thickness, and expected degradation rates. Information is available on corrosion rates for carbon and stainless steels in soil systems [Romanoff, 1957; Gerhold, 1981; Sullivan, 1989].

The failure time must be input for each container in units of years. Upon entering this submenu, the code prints the current values for failure times and allows the user to change each one of these times independently or set a series to a single value.

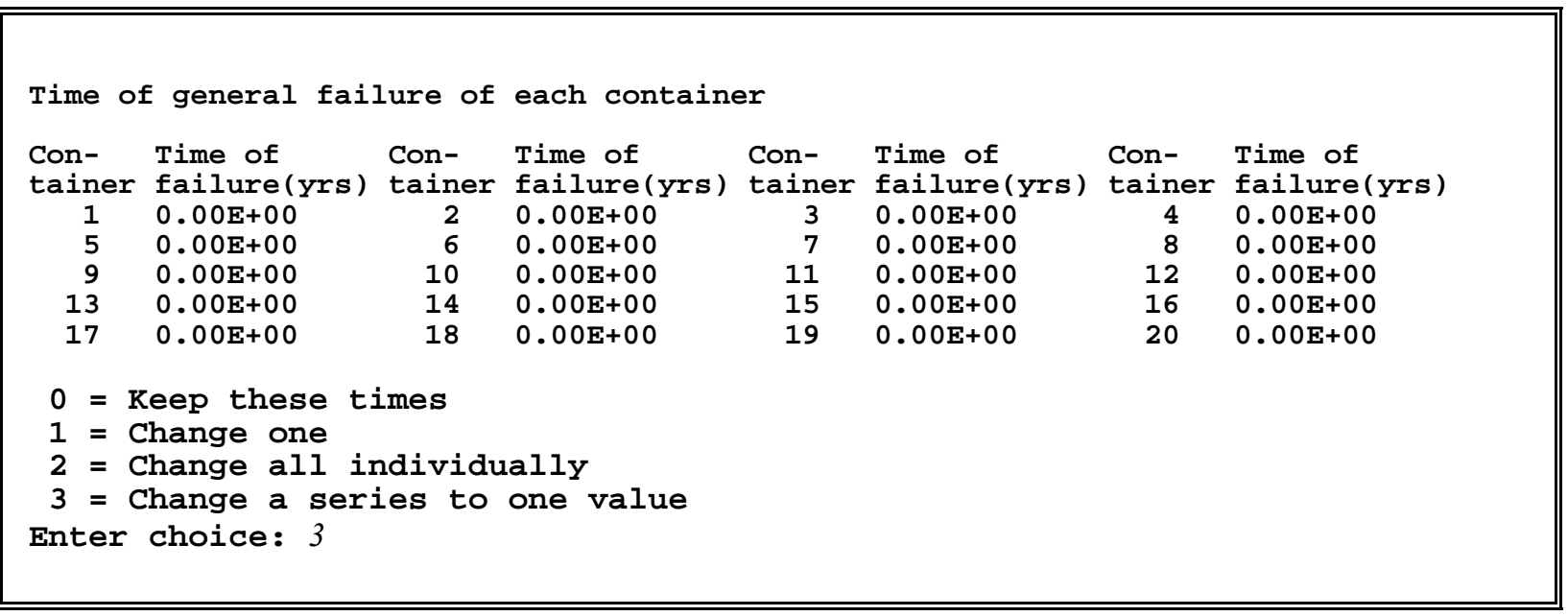

In changing a single value, the code asks for the node number and the failure time. When changing a series to one value, the following instructions are provided on the screen when running 
DUSTINMS. In the example, the user has previously specified that there are 20 containers and then sets the failure time of the first 10 containers at 10 years and the last 10 containers at 20 years. This is achieved through input of three values: a) the value for the first container in this sequence; $b$ ) the total number of containers in the sequence; and c) the failure time. After a sequence has been completed, the current failure times are printed and the user is asked if more changes are required.

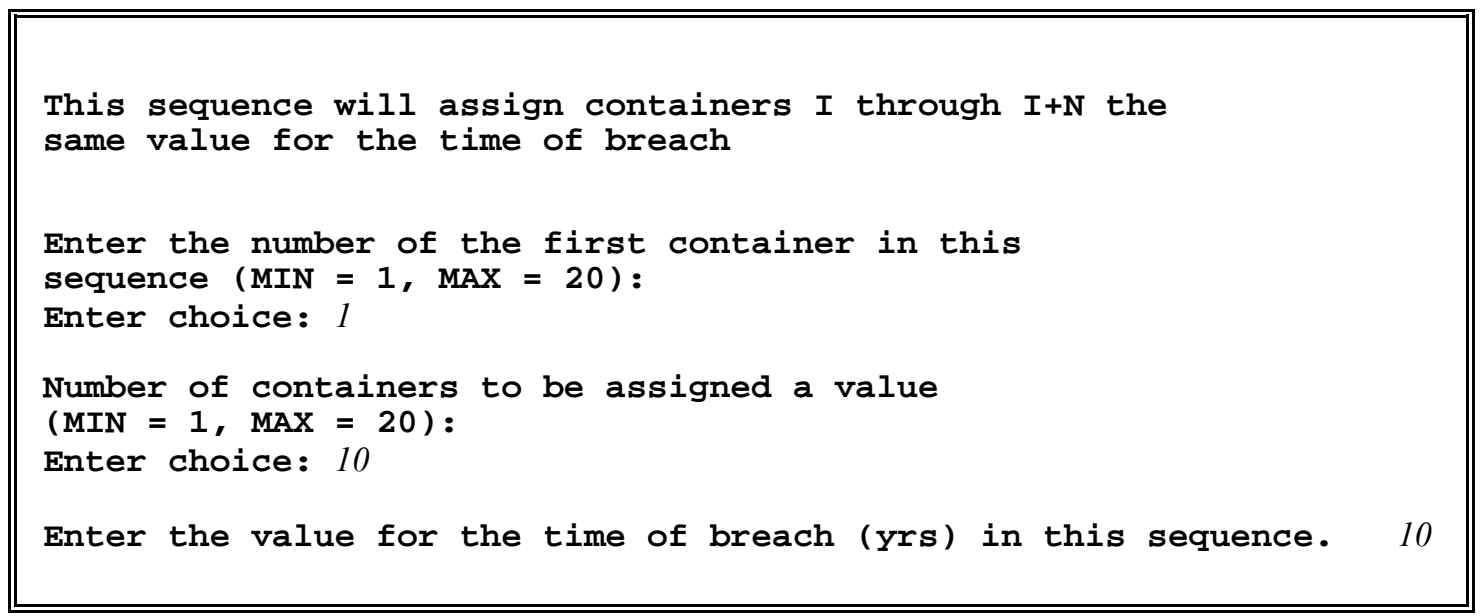

The process was continued for the remaining containers. Output of the current values for failure times follows:

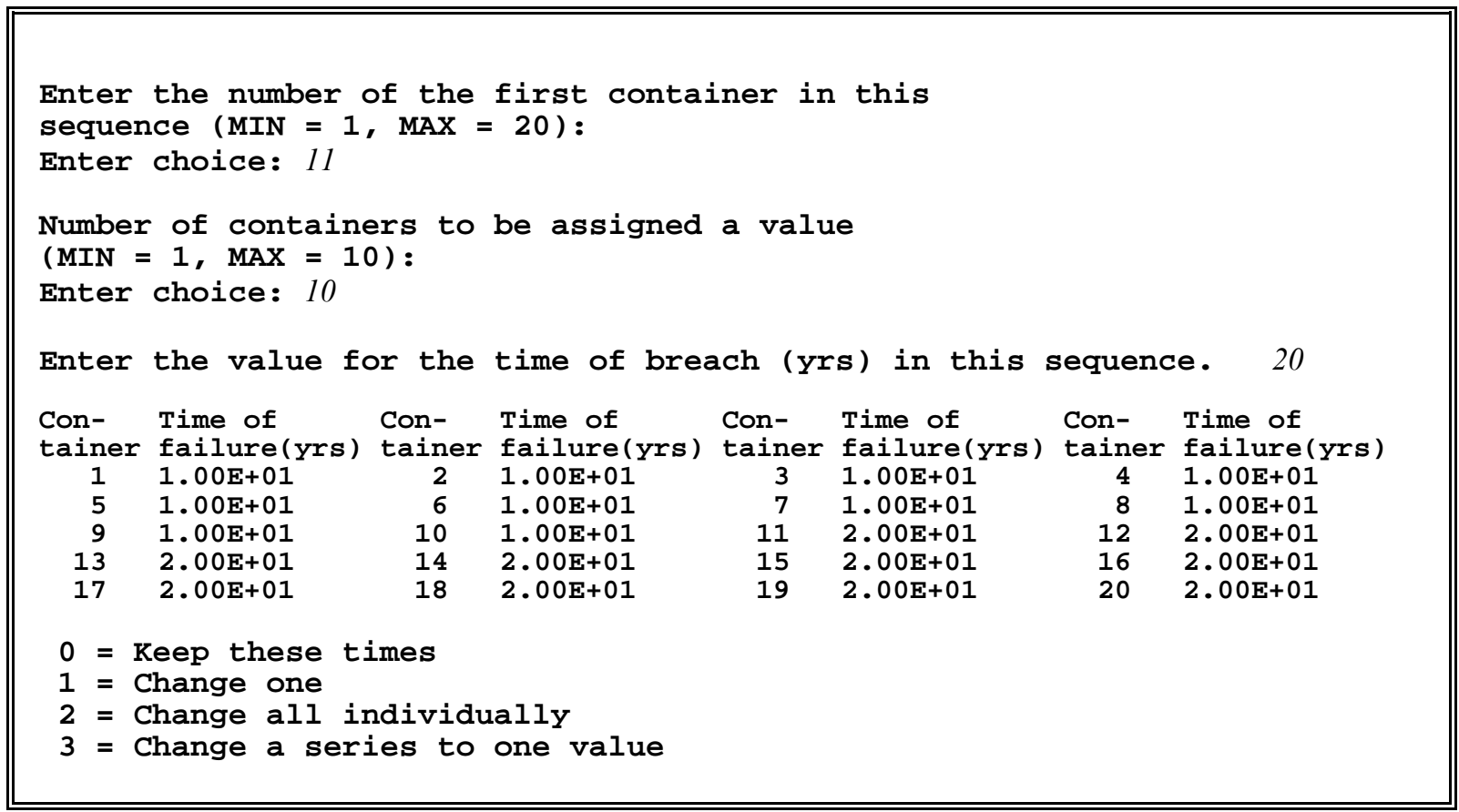




\subsubsection{Location of the Containers}

Each container must be assigned to a unique location. This is accomplished by DUSTINMS in a similar fashion as specifying the failure times. The user is permitted to change each value independently, or change a sequence of values. If a sequence of values are to be created, four values are needed: a) the number of the first container in the sequence; b) the location (cell number) of the first member of the sequence; c) the distance between (number of cells) adjacent containers; and d) the number of values to be assigned in the sequence. The default value for container location is zero. Failure to define this number will cause the DUST-MS code to fail.

The equation used to generate this sequence is presented in the menu as shown below. In the example problem, the user has specified that the first container is in node 20 and the remaining containers are in the 19 adjacent cells. The DUSTINMS code requires that each container location be defined. If the user has not defined all locations the code returns to the location specification menu and requires further input.

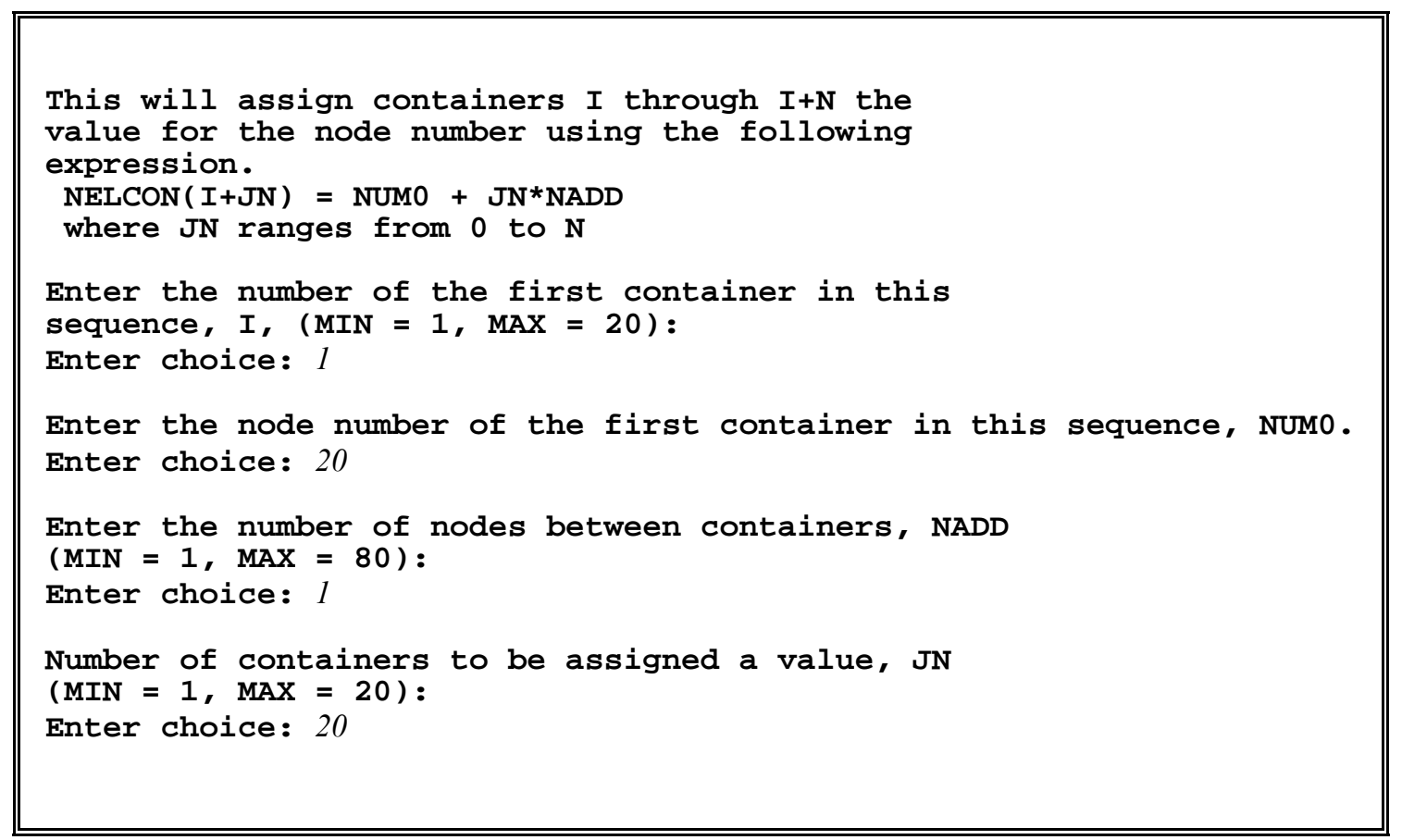

Upon completion of the input, the values are translated into cell (node) numbers and the results are printed for review. 


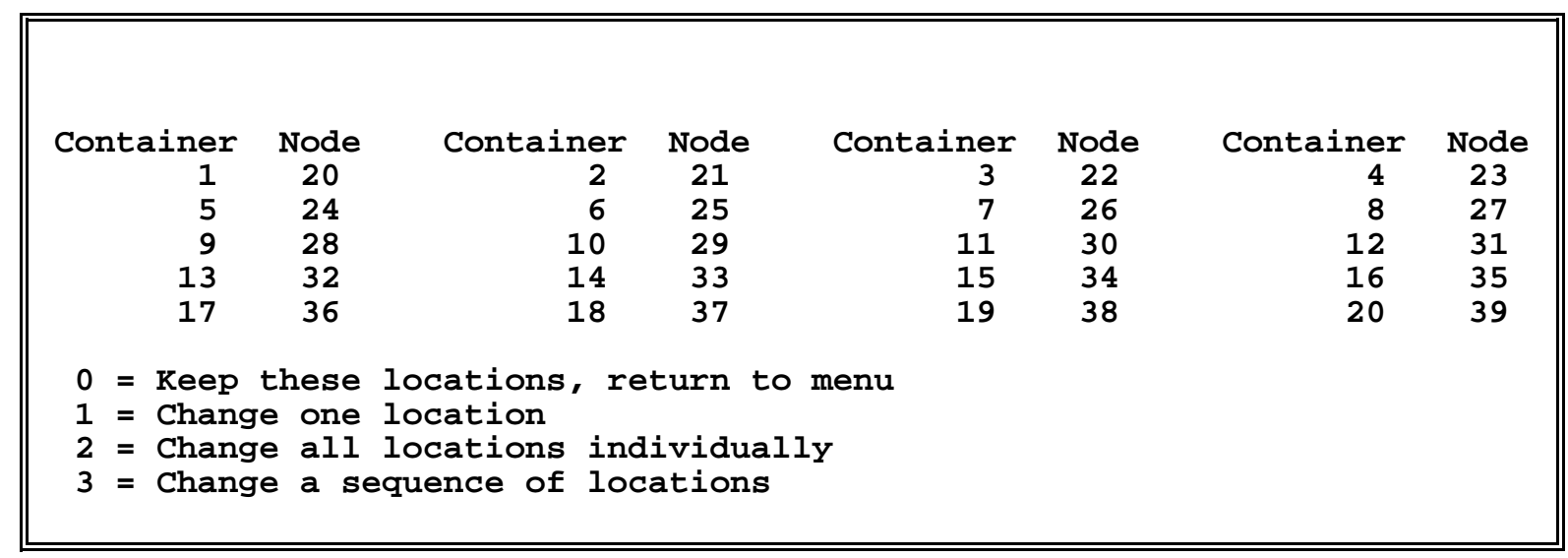

\subsubsection{Number of Failure Types}

Localized failure can be modeled in DUST-MS. The data in the model has been specialized to pitting corrosion but it may be generalized to provide an early localized failure for any process. Up to 20 different localized failure rates may be specified. If localized failure is not desired leave this value equal to the default value, 0 .

\subsubsection{Corrosion Model Flag}

All containers must have a specified time to failure. However, each container type may also fail due to local corrosion. The choice is specified through setting this flag to 1 for local corrosion. This is used only if the number of failure types is non-zero. The structure of the input routine for this value is identical to that for specifying the time to failure and will not be repeated. This is useful when the user desires to model a portion of the containers failing by local corrosion and the remainder by a general failure time. The code user could specify more than one container type and require that one container type does not undergo local corrosion by setting this flag to zero.

\subsubsection{Localized Failure Parameters}

The model for localized failure has been presented in Section 2.4.2. The model requires six parameters (the number of penetrating pits per container, the area of the container, the area scaling factor, the container thickness, and the two empirically determined rate parameters used to describe pit growth). Suggested values for these parameters are presented in Appendix D. The DUSTINMS code permits the user to change every single variable independently through the use of the submenus. Upon entering this submenu, the following instructions are printed. 


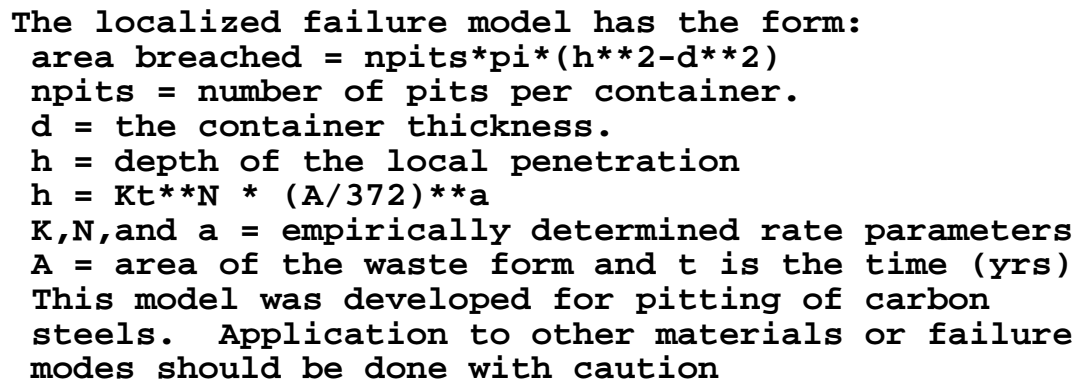

The current values for these parameters are then printed and the user is asked if a change is requested.

\begin{tabular}{||llllllr||}
\hline \hline & & & & \\
Thick & $\mathbf{N}$ & $\mathrm{K}$ & Area & A-scale & No. of Pen. & Index \\
$0.00 \mathrm{E}+00$ & $0.00 \mathrm{E}+00$ & $0.00 \mathrm{E}+00$ & $0.00 \mathrm{E}+00$ & $0.00 \mathrm{E}+00$ & $0.00 \mathrm{E}+00$ & 1 \\
$0.00 \mathrm{E}+00$ & $0.00 \mathrm{E}+00$ & $0.00 \mathrm{E}+00$ & $0.00 \mathrm{E}+00$ & $0.00 \mathrm{E}+00$ & $0.00 \mathrm{E}+00$ & 2 \\
Do you wish to change any of these parameters $(0=$ No, $1=$ Yes $)$ & \\
Enter choice: 1
\end{tabular}

The code then prints out the container types that require localized corrosion parameters and asks which container type will receive changes.

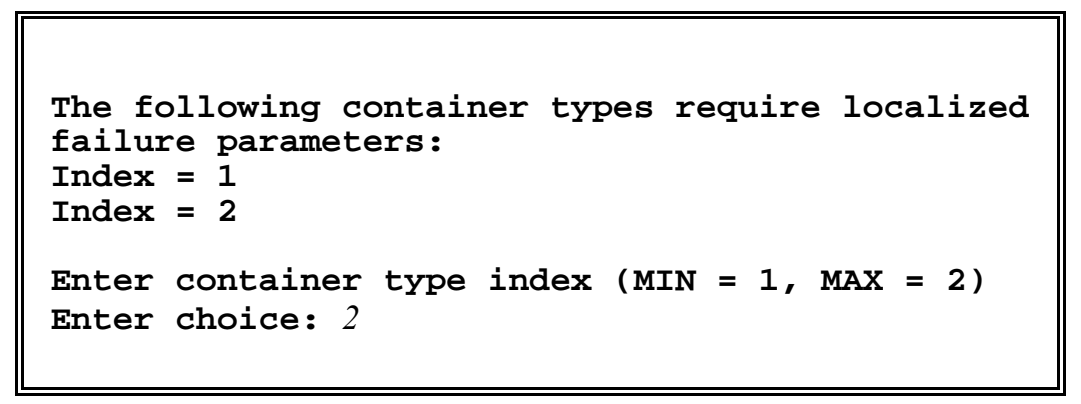

Here container 2 receives the changes. The code then prints out information on the input variables in the failure parameter menu. A full discussion of these variables can be found in [Sullivan, 1989]. 


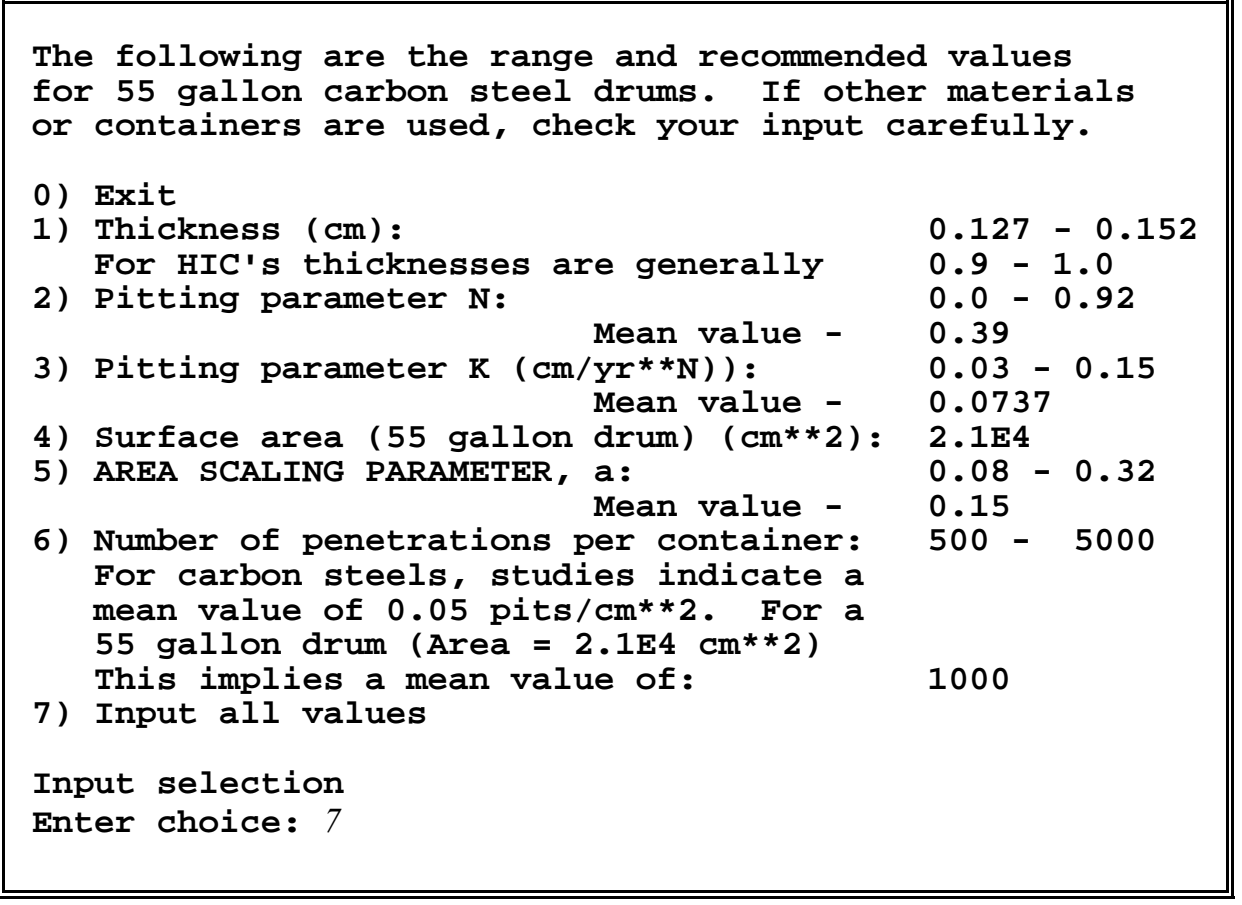

In this case, all parameters will be changed. The code then prints the following and requests the input for all parameters on one line separated by spaces.

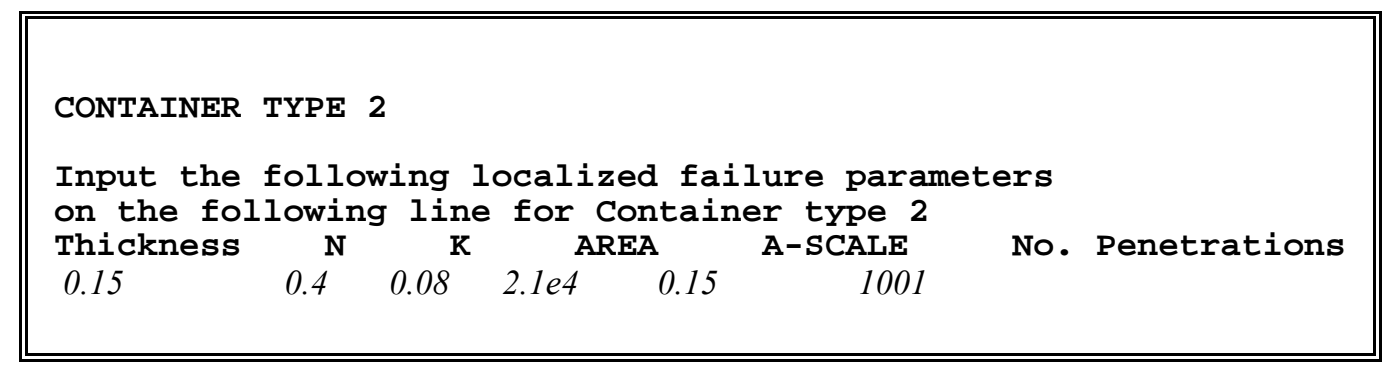

If the change was to be made only to a single parameter, the code prints the parameter name and asks for the input value. It then returns to the parameter menu and asks if another parameter is to be changed. In the example, the code asks if the user wants to continue data entry. If further input is requested, the code asks which material is to receive changes. If input of local failure parameters is complete, control is returned to the container parameter menu (i.e., the menu containing information on number of containers, time of failure, etc.). Upon returning to that menu, the values for the rate parameters are printed. 


\begin{tabular}{||llllllr||}
\hline Thick & N & K & Area & A-scale & No. of Pen. & Index \\
$1.50 \mathrm{E}-01$ & $3.90 \mathrm{E}-01$ & $1.00 \mathrm{E}-01$ & $2.10 \mathrm{E}+04$ & $1.50 \mathrm{E}-01$ & $1.00 \mathrm{E}+03$ & 1 \\
$1.50 \mathrm{E}-01$ & $4.00 \mathrm{E}-01$ & $8.00 \mathrm{E}-02$ & $2.10 \mathrm{E}+04$ & $1.50 \mathrm{E}-01$ & $1.00 \mathrm{E}+03$ & 2 \\
& & & & & & \\
\hline
\end{tabular}

\subsubsection{Assignment of Localized Failure Parameters to Each Container}

If more than one set of container types is requested in Menu 8.4, the user must specify the container type for each container. This assigns local container failure parameters to each container. The procedure is similar to that used in specifying times to failure, the user can change any single container type or a sequence of container types. Upon entering this menu, the code prints out the existing values and requires reassignment to non-zero values.

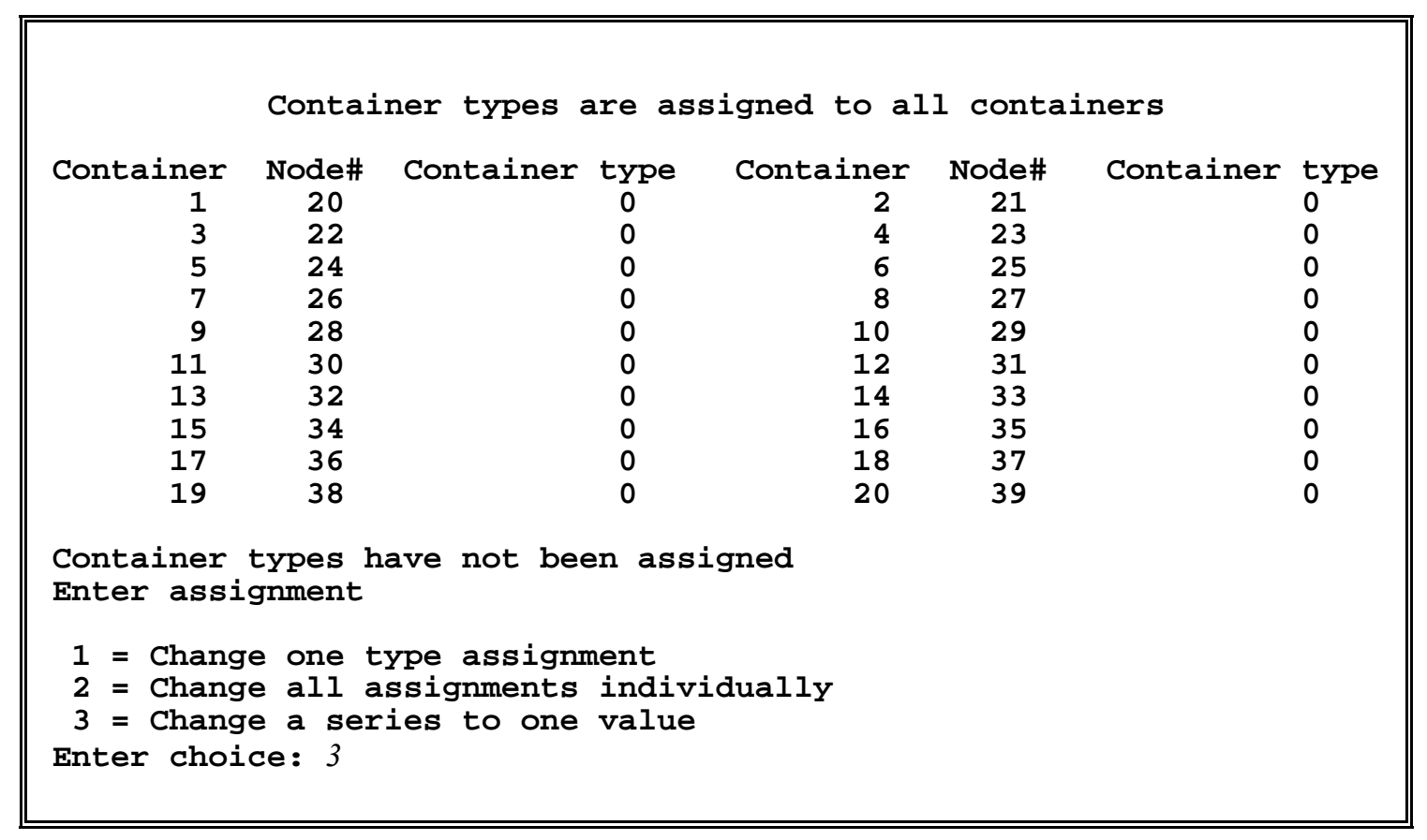

To reassign a series of container types three input variables must be supplied, the container number of the first container in the series, the number of containers in the series, and the container type for the series. In this example, containers 1 - 10 are given type 1 failure parameters and containers $11-20$ are specified as type 2 containers. 


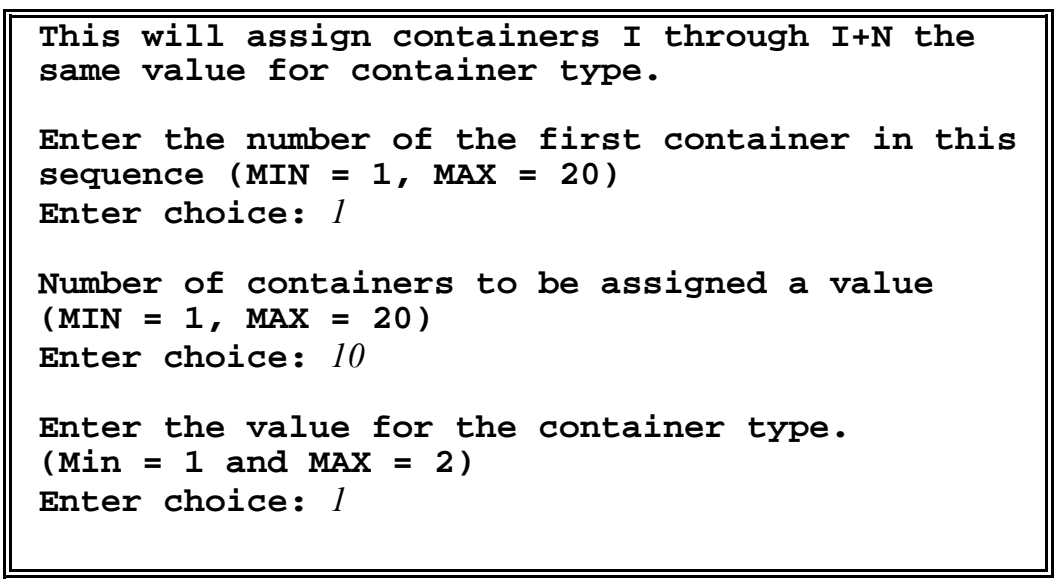

At this point, only 10 of the 20 containers have been reassigned. The DUSTINMS code automatically asks for a continuation until all 20 containers are assigned a non-zero value.

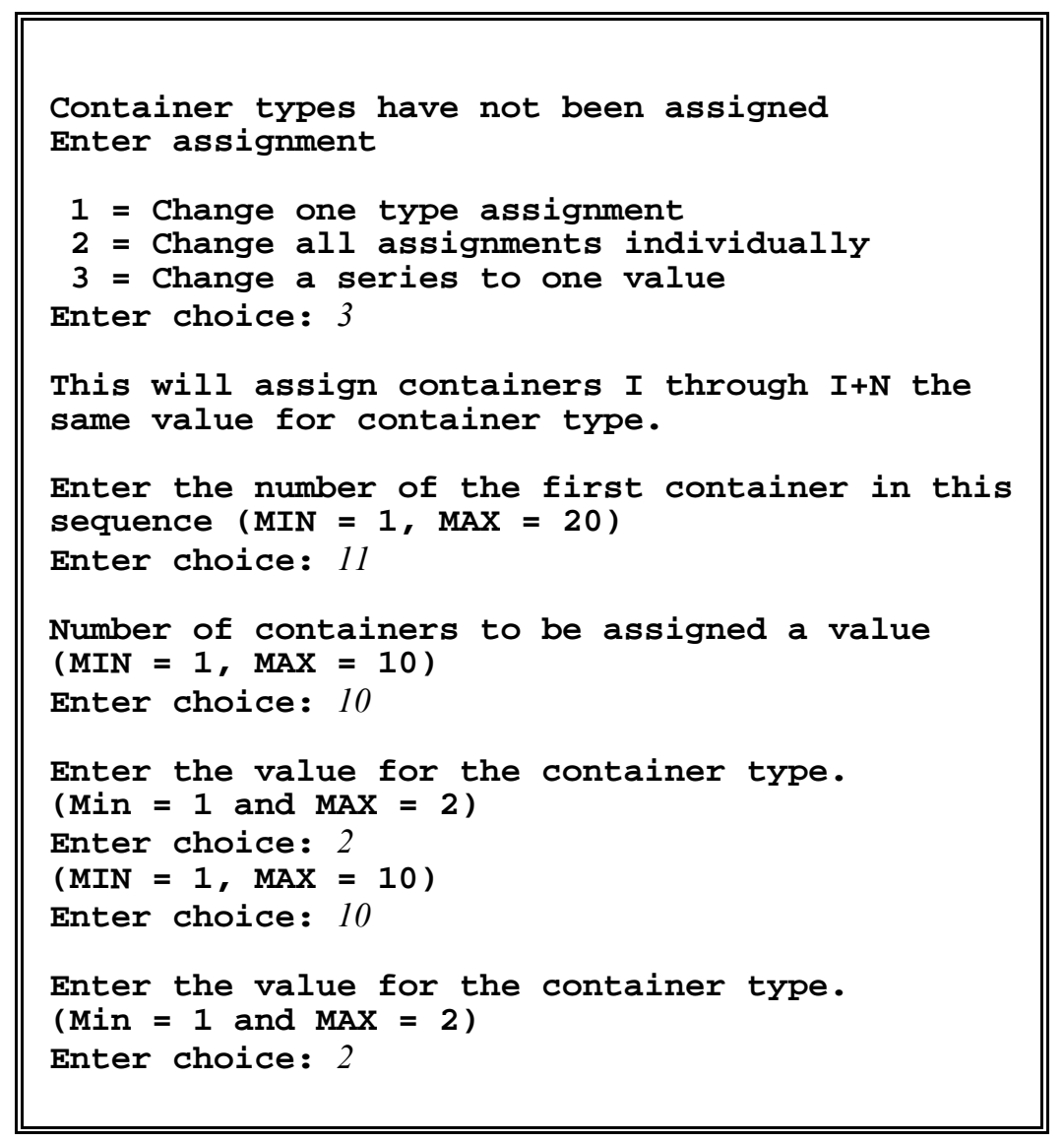

After the container assignments are complete, the values are printed for review. 


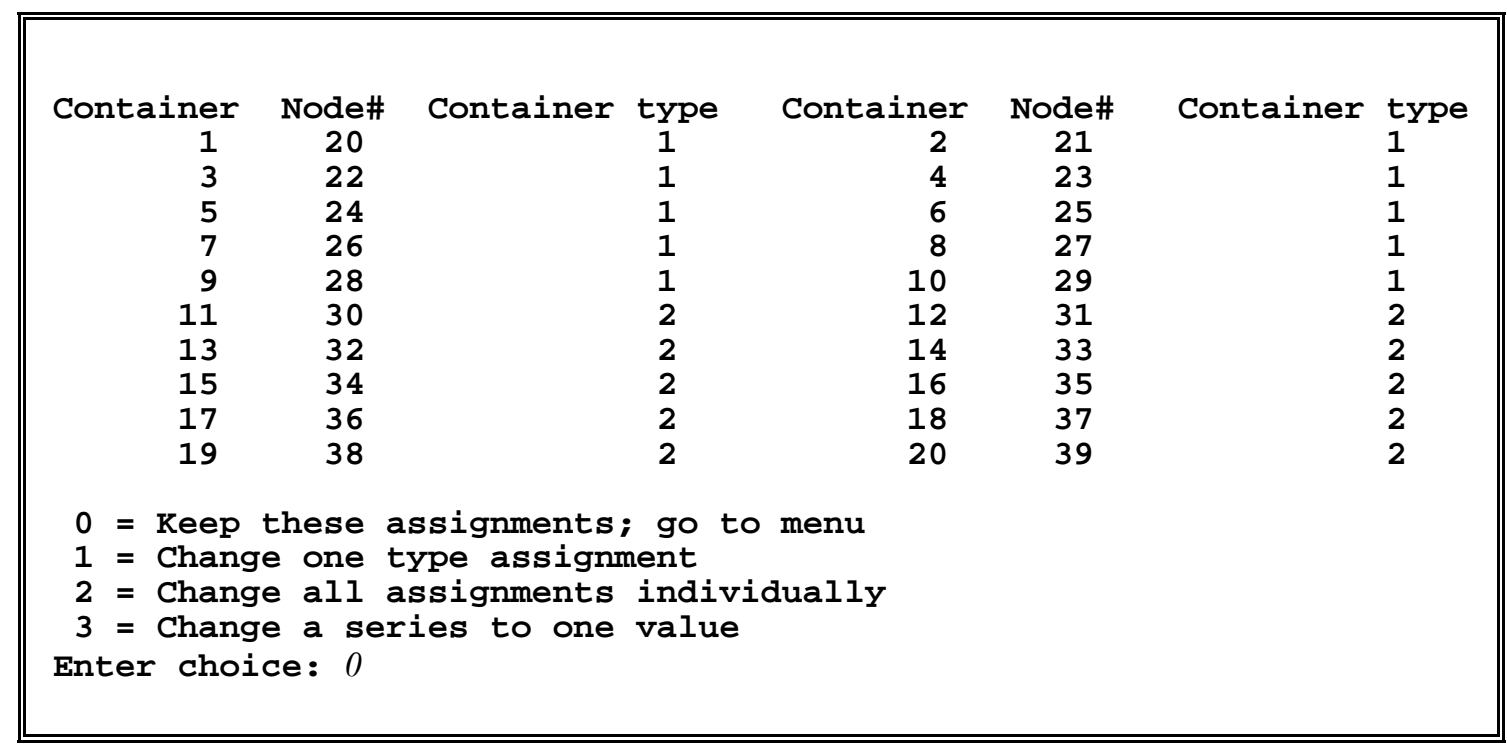

\section{9}

\section{Waste form Parameters}

The DUST-MS computer code simulates a waste form in every control volume that has a container (the maximum number of containers is 300). Each waste form is permitted to have a unique inventory. However, the code permits only twenty different waste form types to be modeled. Each waste form type is represented by a unique set of release rate parameters. For each waste type, each isotope can have unique release parameters.

DUST-MS allows four mechanisms to control release. Rinse with partitioning, diffusion release, uniform release and solubility limited release. Diffusive releases may be simulated with analytical models which assume uniform initial concentration, zero boundary concentrations and do not account for ingrowth due to decay. Diffusive releases are dependent on the geometry and dimensions of the waste form. This requires additional input. Either rectangular, cylindrical, or spherical can be modeled. In addition, a finite difference representation of the waste form in onedimension for any of the above geometries is permitted. The finite difference waste form combines all three release mechanisms into a unified model and permits direct handling of ingrowth.

The waste form parameters menu is:

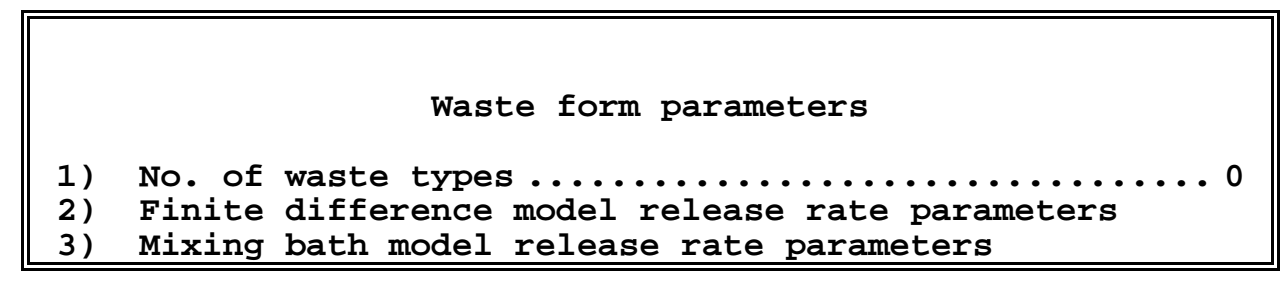




\begin{tabular}{|ll||}
\hline 4) Initial amount of $\mathrm{XXXXXXX}$ in each container \\
6) Waste type assignment to each container \\
Enter choice:
\end{tabular}

\subsubsection{Number of Waste Types}

The number of waste types is the number of waste forms having unique release rate parameters. The maximum value permitted in DUST-MS is 20 .

\subsection{2}

Finite Difference Model Release Rate Parameters

Upon requesting to alter the finite difference release rate parameters, the following menu is printed.

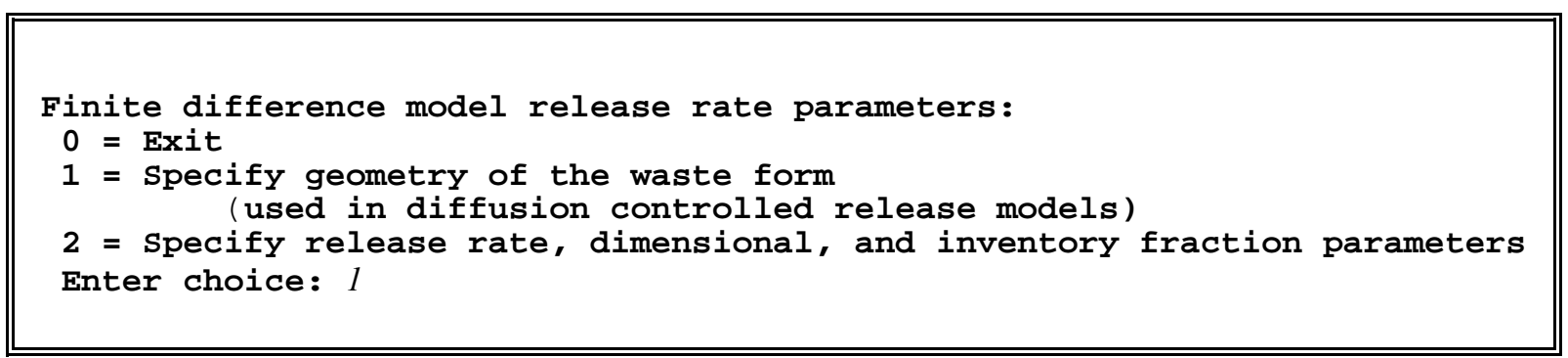

The default choice is rectangular geometry with an analytical solution. If other diffusion models are required, the geometry flags need to be redefined.

If the geometry is to be redefined, the code prints out existing values for the flags, and the geometry parameters (radius, volume, and half-width), and asks if a change is desired. If changes are desired for a particular waste form the following menu is displayed.

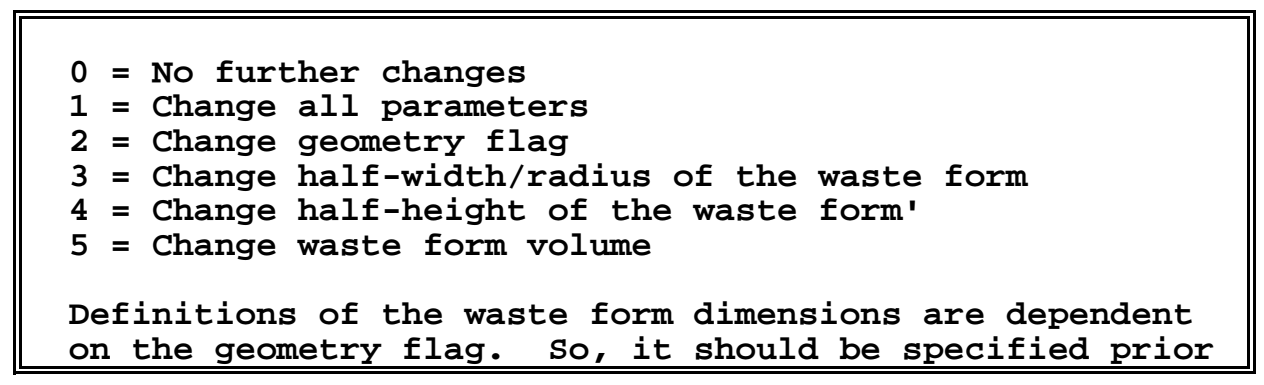




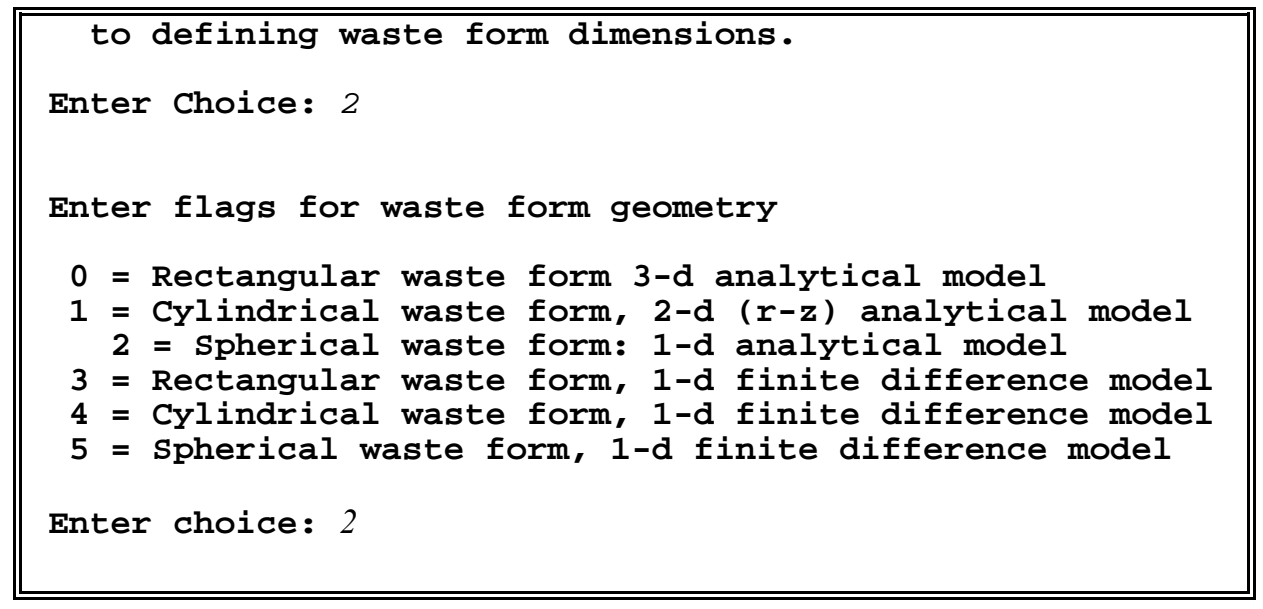

In this example, the user has selected the analytical release model from a spherical waste form. In general, the radius of the waste form would be defined next to complete the geometry definition for this waste type.

At this point, the code prints the values for the flags and provides the user with an opportunity to make further changes or return to the menu which accesses the release rate parameter menu.

Item 3 is a dual purpose variable. For cylindrical and spherical waste forms, it is the radius. For rectangular waste forms it is the half-width. This variable is used for predicting diffusion release and defining the waste form finite difference geometry. The analytical release models require the dimensions of the waste form. In DUST-MS, these are specified by supplying the radius for spherical waste forms, the radius and volume for cylindrical waste forms, and the half-width, halfheight, and the volume combine to define the geometry of rectangular waste forms.

Item 4 , is the half-height of a rectangular waste form. This variable is not used for spherical or cylindrical waste forms.

Item 5 , is the volume of the waste form. This value is calculated by the code for spherical waste forms. For spherical waste forms, the volume is calculated as $4 \pi \mathrm{r}^{3} / 3$, where $\mathrm{r}$ is the radius. If the finite-difference model waste forms are specified and the waste form volume is input as zero, the volume is calculated from the radius (or half-width) as follows. For cylindrical waste forms, the volume is calculated assuming a unit height, therefore, it is $\pi r^{2}$ where $r$ is the radius. For rectangular waste forms, the volume is calculated assuming a unit area, therefore it is $2 r$, where $r$ is the halfwidth. The volume of the waste form determines the concentration in the waste form. Therefore, it effects the release due to solution feed back effects. In the finite difference waste form models, release to solution is dependent on the gradient of the concentration at the edge of the waste form and in solution.

Caution must be exercised to insure that consistent values are used for both the volume of the waste form and the volume of the contacting solution. The volume of the contacting solution is the 
area of the facility multiplied by the size of the computational cell and the moisture content in the cell. For example, assume that the user defines the area of the facility to be $1 \mathrm{~cm}^{2}$, the computational cell to be $1 \mathrm{~cm}$ long, and the moisture content of 0.2 . Therefore the volume of solution in the contacting cell is $0.2 \mathrm{~cm}^{3}$. If release from a 200 liter drum (volume $210^{5} \mathrm{~cm}^{3}$ ) is modeled, diffusive releases will be very low because the amount of mass required to equilibrate the concentration in the $0.2 \mathrm{~cm}^{3}$ of solution is small compared to the total mass in the $210^{5} \mathrm{~cm}^{3}$ waste form.

At this time, the example below indicates that release rate parameters require definition.

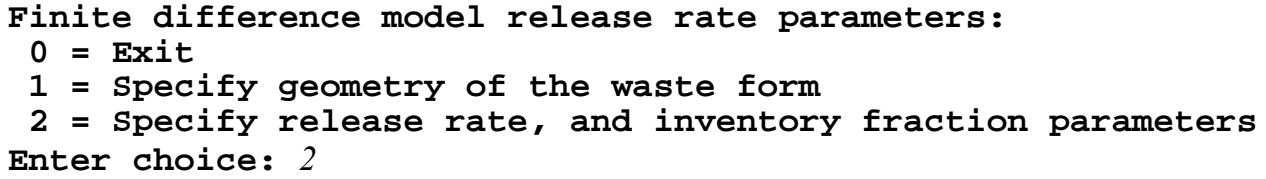

The release rate parameter menu follows. Again, the user can change all parameters independently through the menu. Upon entering this menu, the current values are printed and the user is asked if changes are desired for one or radionuclides. Also, the changes to a specific waste type must be specified. In this example, two waste types (Menu 9.1) have been requested and the FD transport model is specified.

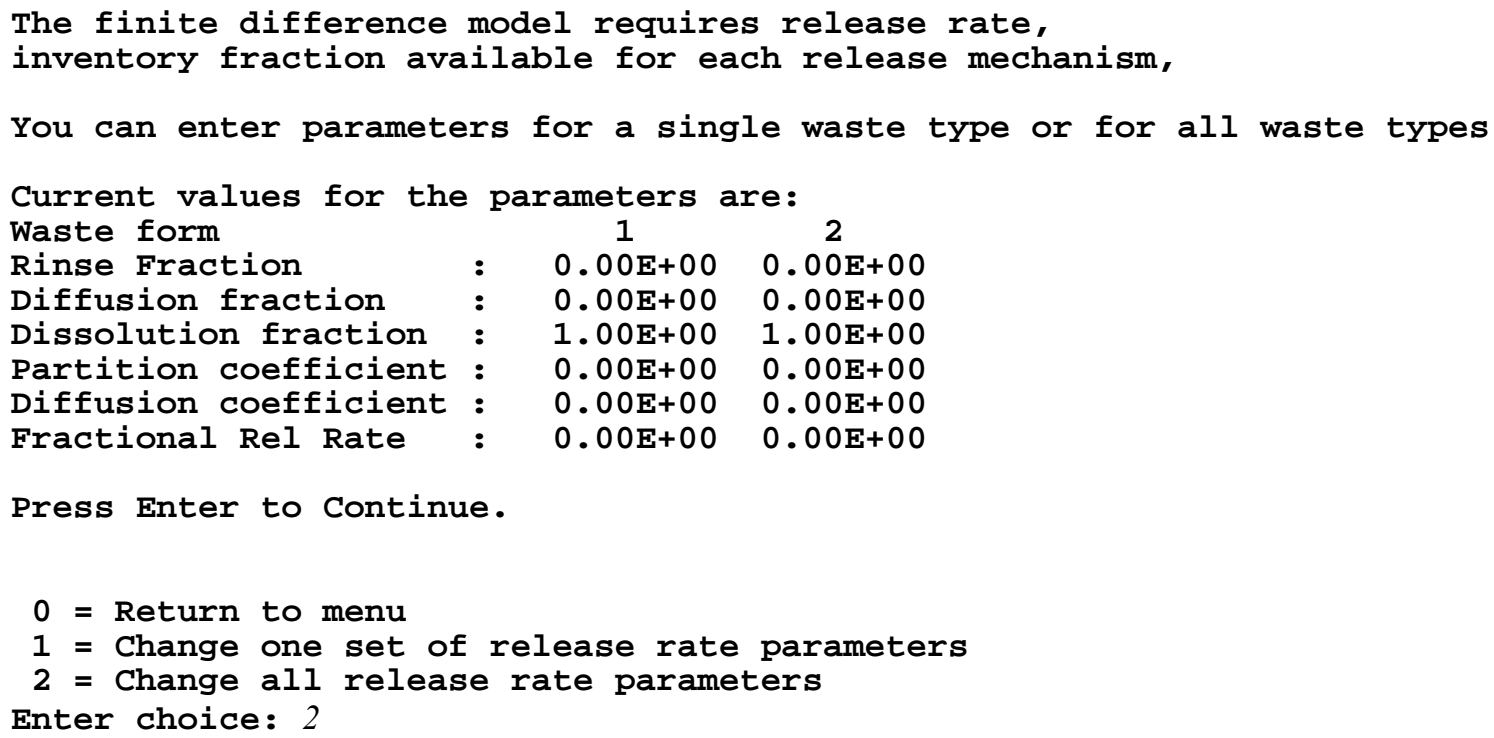


The code will cycle through all of the different waste types asking for changes to the release parameters. In this example, waste type 1 has been defined and the code asks for changes to waste type 2 .

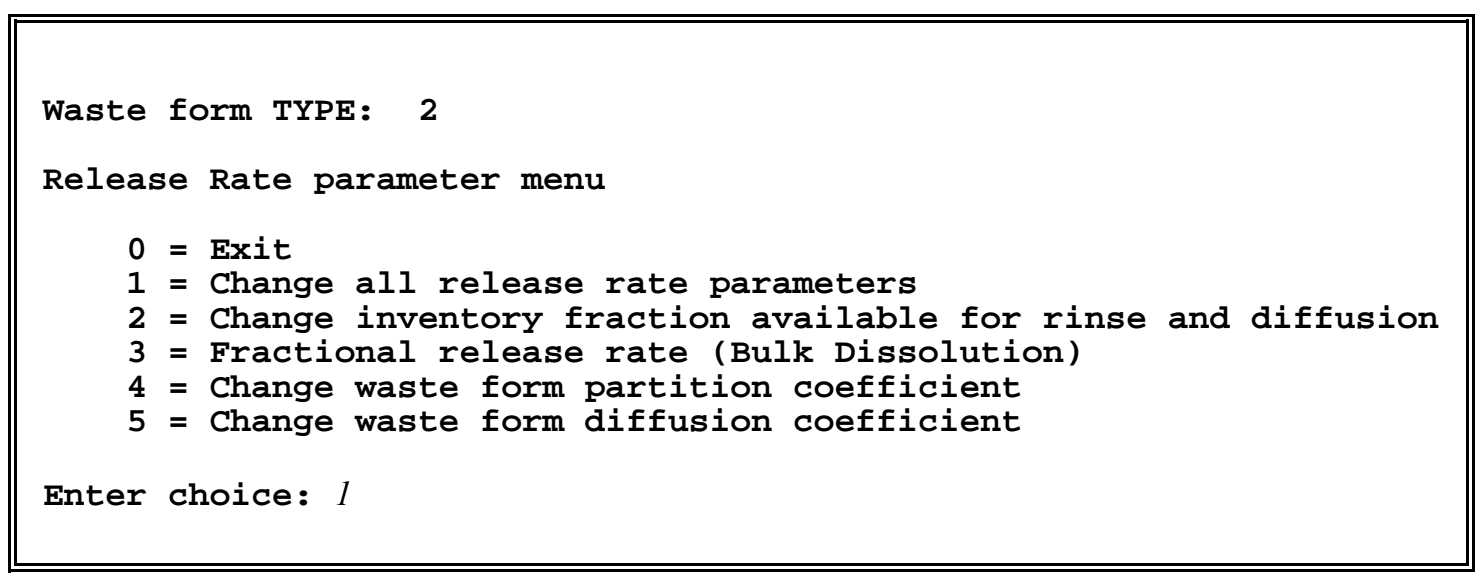

The first time through this menu it is necessary to define all of the parameters. There are three release mechanisms: rinse, diffusion, and uniform. For the analytical release models, IDIFF $=0,1$, or 2, each of these is independent. The amount of mass available for release by each mechanism is the total mass in the waste form (Menu 9.5) multiplied by the fraction of mass available for each mechanism. These are input as the fraction available for rinse and the fraction available for diffusion. The fraction available for uniform release is determined by requiring that all three sum to 1 .

Item 3 on the menu, the fractional release rate, is the fraction of the uniform release fraction that is released per year. The definition of the fractional release rate changes depending on the choice of the analytical or finite-difference waste form release models. For the analytical models, the fractional release rate is the uniform value specified in input. For example, if a waste form had an initial inventory of 1 Curie and $10 \%$ of the inventory was available for uniform release, a fractional release rate of 0.05 per year would release $0.005 \mathrm{Ci} / \mathrm{yr}$ (this value would be adjusted to account for decay) due to uniform release. For the finite-difference models, the fractional release rate is the fraction of the original length that dissolves per year. For example, for a waste form with a radius of $20 \mathrm{~cm}$, a fractional release rate would of $0.05 / \mathrm{yr}$ would dissolve $1 \mathrm{~cm} / \mathrm{yr}(20 \mathrm{~cm}$ multiplied by 0.05 ). The amount released depends on the geometry. For rectangular geometry, release would be $1 / 20$ of the inventory or $0.005 \mathrm{Ci} / \mathrm{yr}$ identical to the analytical models. For spherical and cylindrical waste forms, the amount release changes in time due to the changing amount of volume dissolved. For example, for a spherical waste form with an initial radius of 20 $\mathrm{cm}$, removal of the outer $\mathrm{cm}$ removes $14.2 \%$ of the volume as compared to $5 \%$ of the volume for the rectangular waste form. Therefore, the first year release would be $.0142 \mathrm{Ci} / \mathrm{yr}$.. Additional releases would occur due to the diffusion and rinse mechanisms. 
Item 4, the waste form partition coefficient, can be used to represent control of release by reversible chemical interactions with the waste form, for example sorption on dewatered ion exchange resins. If this value is zero, the entire rinse mass (total mass multiplied by rinse fraction) is released into solution upon breach of the container subject to solubility limits. If the partition coefficient is non-zero, releases from this waste form can be negative. The model performs a mass balance and determines the distribution of contaminants subject to the partition coefficient. Therefore, contaminants in solution may be removed causing a negative release for this waste form. When using the partition coefficient the code user must be extremely careful so as not to account for sorption effects twice as there is also a distribution coefficient in the transport model. This is discussed in detail in Section 2.5.2.

Item 5, waste form diffusion coefficient, is used when the diffusive release fraction is nonzero. The code has analytical solutions for finite-sized cylindrical and rectangular waste forms, see Section 2.5.3. Values for the diffusion coefficient depend on the waste stream and solidification agent. The Technical Position on Waste form [Lohaus, 1991] requires that the diffusion coefficient be smaller than $10^{-6} \mathrm{~cm}^{2} / \mathrm{s}$ for solidified wastes. A collection of diffusion coefficient values is presented in [Sullivan, 1989].

Diffusive releases depend strongly on the waste form dimensions. For a fixed value of the diffusion coefficient, larger waste forms will have a lower cumulative fractional release as compared to smaller ones. Therefore, it is crucial to input the proper dimensions. In many cases, in order to improve the numerical accuracy of the model, it may be necessary to model a single waste form using several control volumes. In this case, the waste form dimensions must be input as the true dimensions. To properly model the total release, the waste form mass should be evenly divided among all control volumes representing the waste form. For example, if 10 control volumes were used to model a cylindrical waste form having a height of $50 \mathrm{~cm}$, a radius of $25 \mathrm{~cm}$ and with an initial inventory of 40 curies, the proper way to model this would define 10 waste forms all with the same release mechanisms, i.e., all the same waste form type. The radius of this waste form type is $25 \mathrm{~cm}$ and the volume is $9.82 \mathrm{E} 4 \mathrm{~cm}^{2}$. The initial inventory for each of these 10 waste forms is 4 curies.

For rinse release, the dimensions are unimportant in estimating waste form release. Therefore, if a waste form is divided, the initial mass of the contaminant should be divided uniformly between the control volumes. For uniform release, if the analytical diffusion models are selected, IDIFF $=0,1$, or 2 , the dimensions again do not influence release. For the finite-difference model, the geometry does effect the release for the uniform release mechanism. In the finitedifference model, uniform degradation implies a surface reaction that removes the outer layer of the waste form at a constant rate. Therefore, for curved waste forms (cylindrical or spherical) releases are highest initially because the volume removed per unit change in radius is largest initially

The following example requests the properties for waste type 2 which was previously defined as having cylindrical geometry. The user has requested to change all variables. The code prompts the user for each variable and gives the required units. 


\begin{tabular}{|l||}
\hline Waste form TYPE: 2 \\
Fraction of waste's inventory available for SURFACE RINSE: 0.1 \\
Fraction of waste's inventory available for DIFFUSION: 0.8 \\
Waste form partition coefficient (cm**3/gm): 0.0 \\
Diffusion coefficient inside waste form (cm**2/s): $1 e-9$ \\
Radius of cylindrical waste (cm): 25 \\
Volume of waste form (cm^3): $1.67 e 5$ \\
Waste form type parameters complete. \\
\hline \hline
\end{tabular}

Upon completing the input routine, the code prints out the current values for the release rate parameters and asks if further changes are requested.

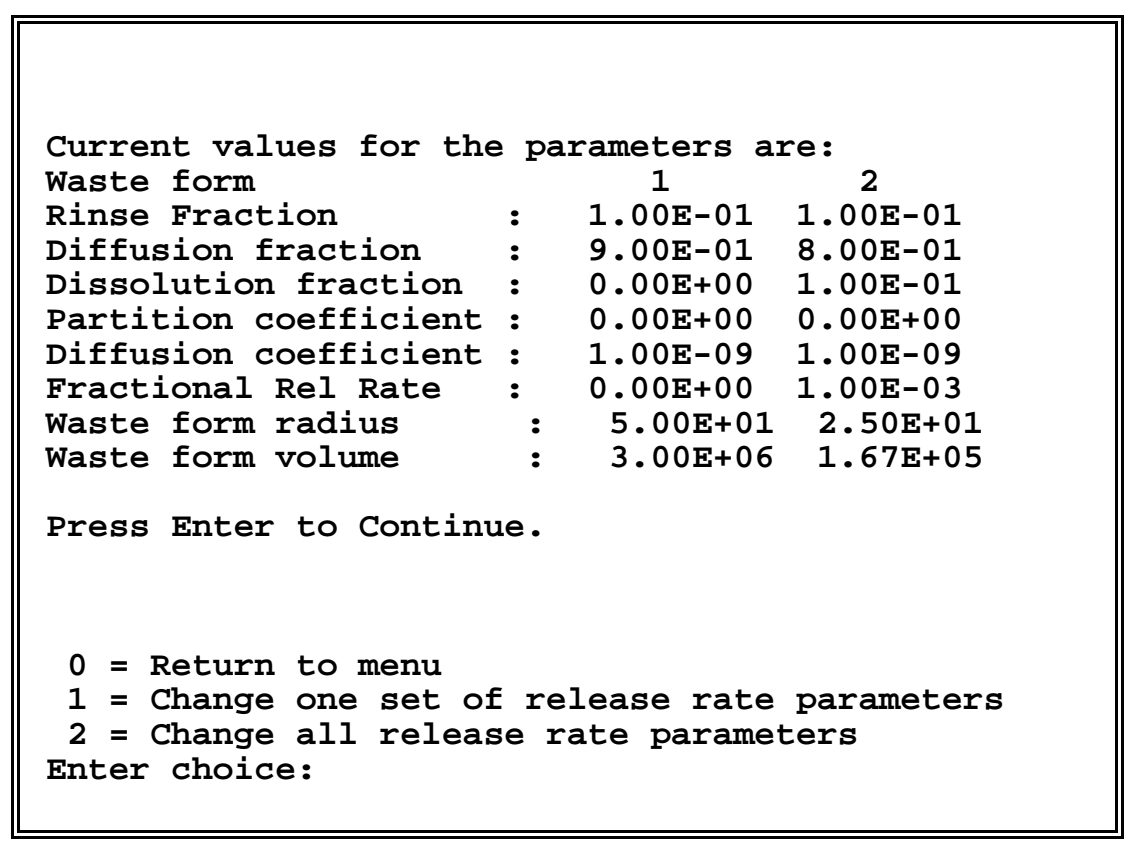

6.9.3 Mixing Cell Model Release Rate Parameters 
The MCMC model is not permitted in DUST-MS. It is a remnant from the DUST

\subsubsection{Initial Inventory}

The user must supply the initial inventory for each waste container. The units for inventory are specified by menu item 1.3. Input can be done for each individual container or a series of waste forms may be assigned an identical inventory. In the following example, the user has previously requested 20 waste containers (Menu 8.1) with the inventory units of curies. The code prints the current inventory (all zero by default) and asks for changes. In this case, a series of inventories will be set to a single value.

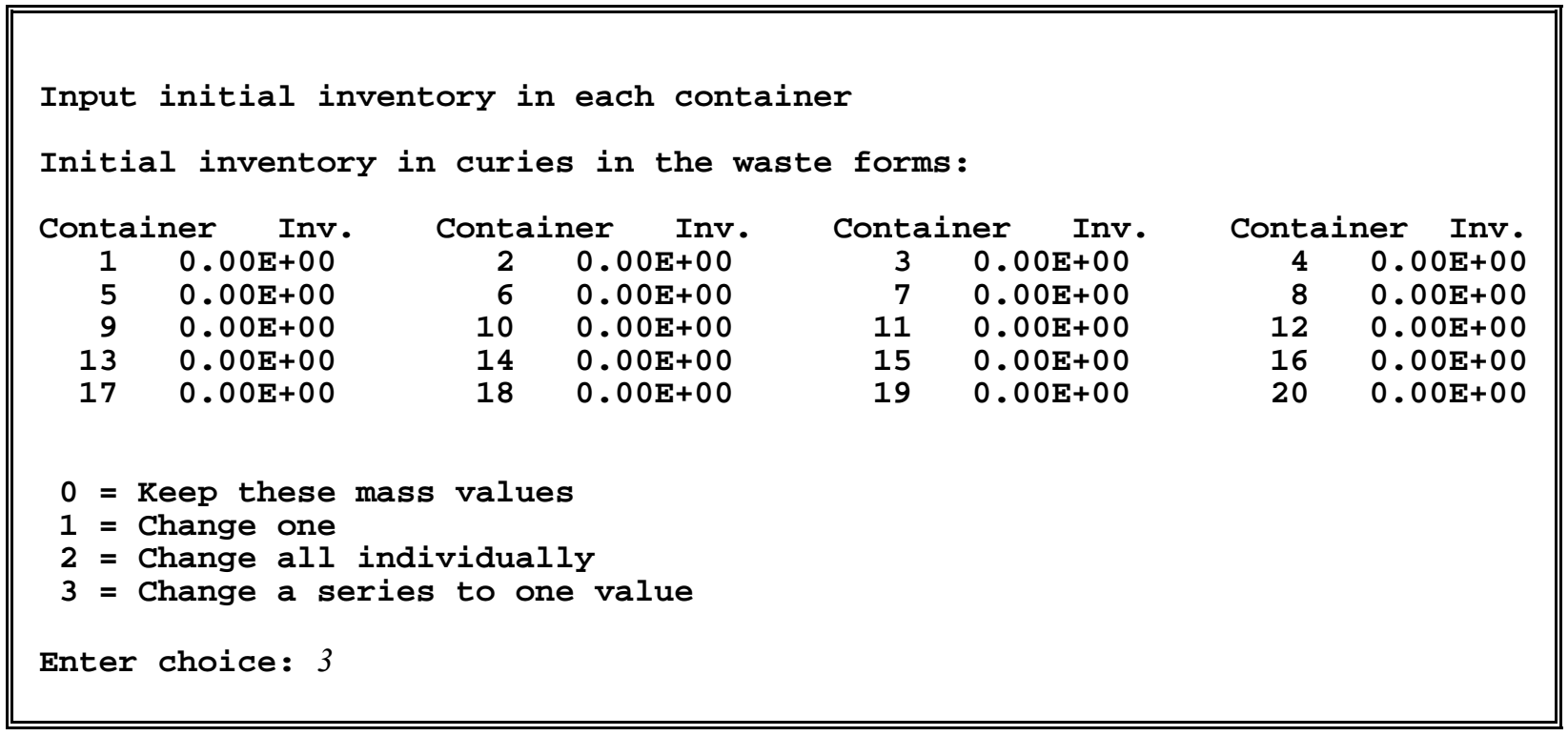

The next few lines define waste containers $1-20$ as having an initial inventory of 10 Curies.

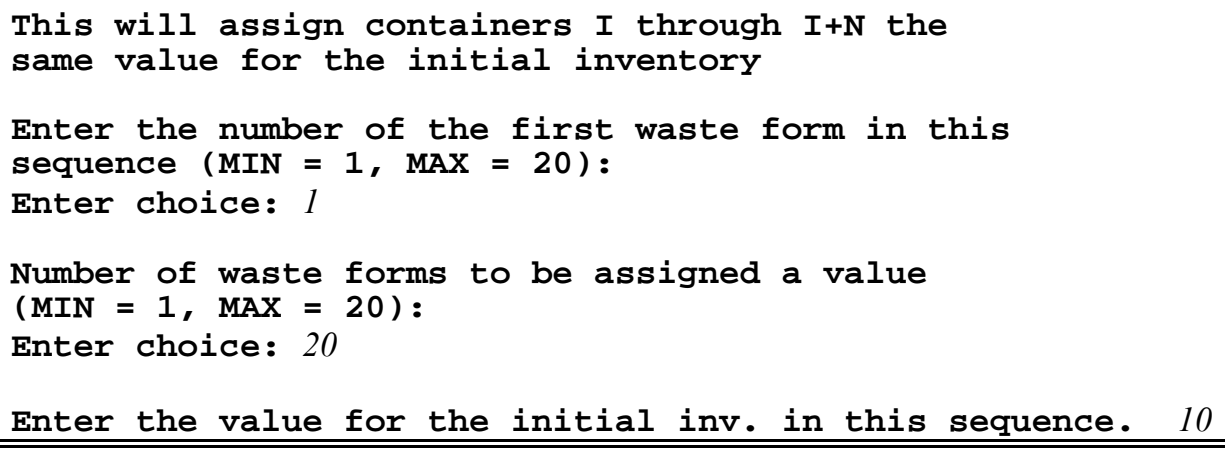


After completing the input, the current values for the inventory are printed and the user is asked if further changes are required.

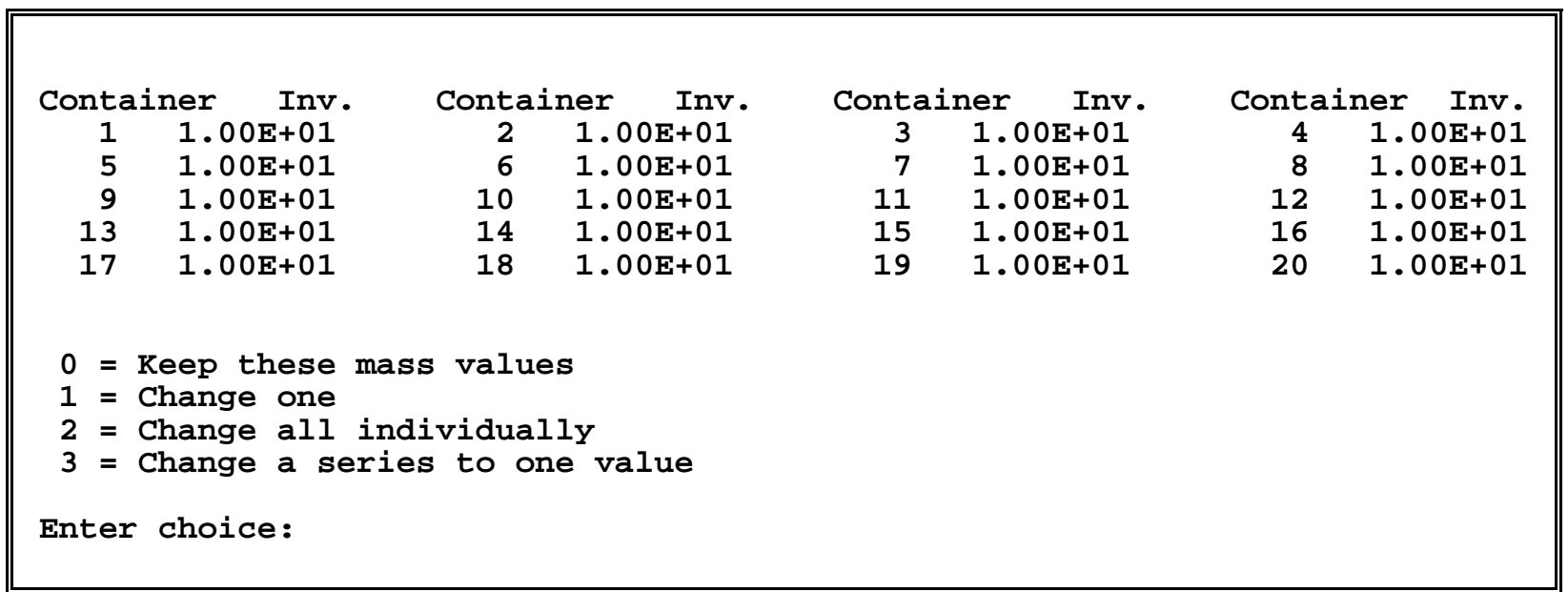

\subsubsection{Waste Type Assignment to Each Container}

This variable acts as a flag that links a set of waste form release rate parameters to a specific container and is required only if more than one set of release parameters are defined. The input routine is similar to that used for inventory assignment. The user has the option of changing a single waste type assignment, changing all waste type assignments individually, or changing a sequence of assignments to a single value.

Upon entering this menu, the code prints the current values and asks the user if further changes are required. In this example, the first 10 containers are assigned waste type 1.

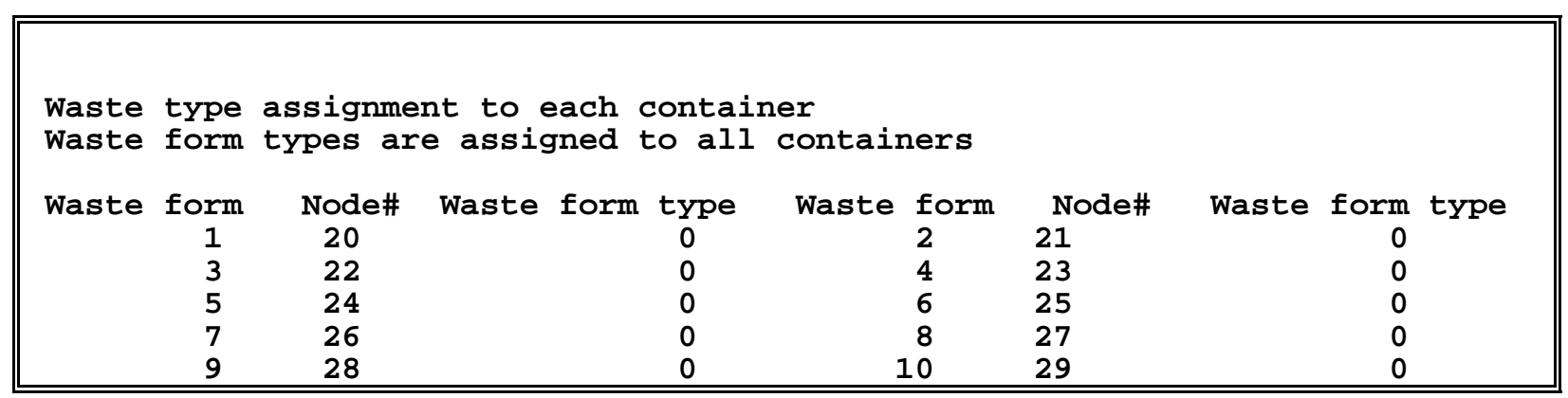




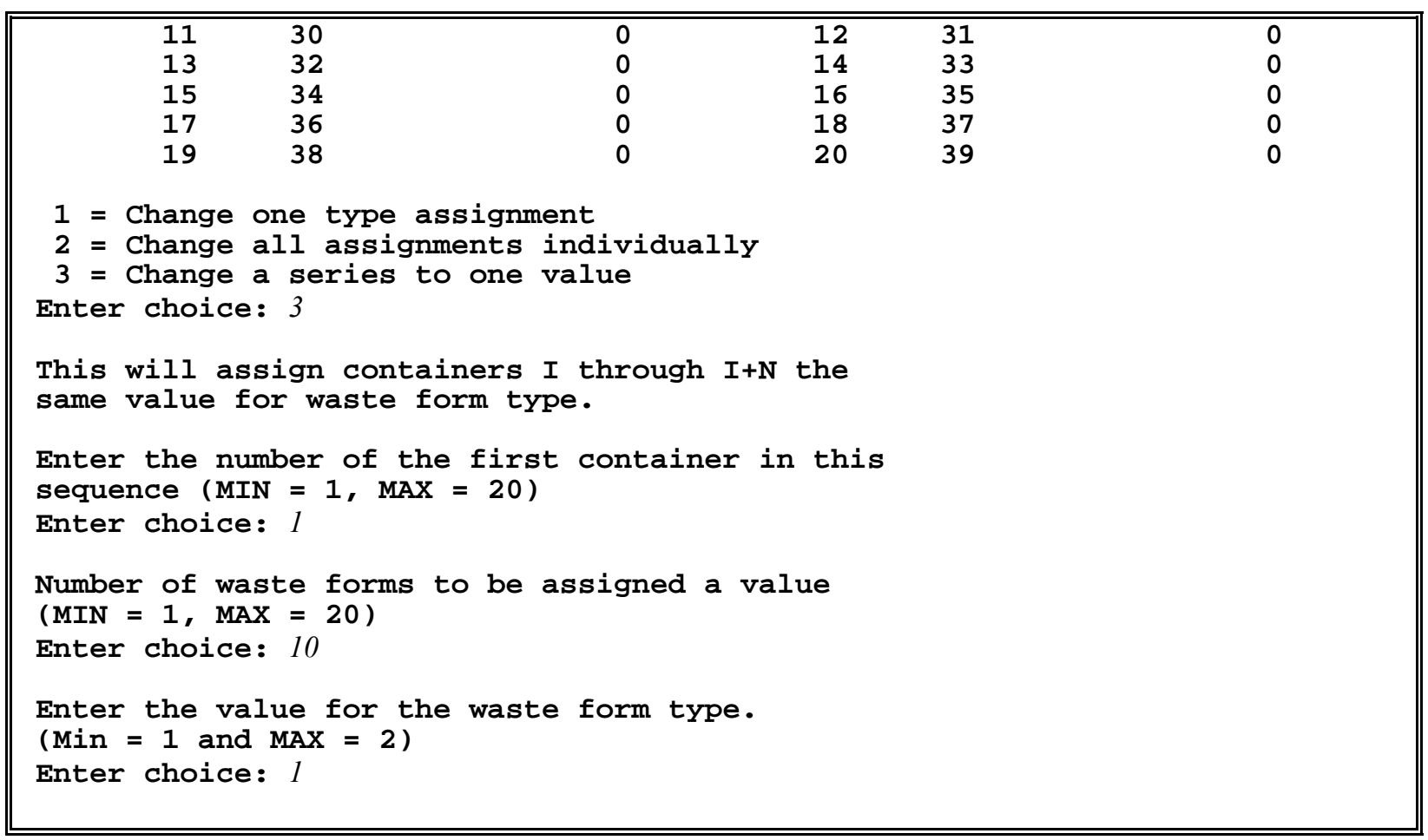

At this point, the program would print out the existing values for waste type assignment and require the user to input more values. The code requires that all waste type assignments are nonzero before allowing the user to return to the waste form menu.

\subsection{External Source/Sink Terms}

The DUST-MS code user can supply an external volumetric source/sink term. This term represents the rate of injection/removal of mass into the volume represented by one finite difference control volume in the system. The external source term is specified as a function of time through tabular input. Values are obtained through interpolation using the table. In the DUST-MS code this term has units of mass $/ \mathrm{cm}^{3} / \mathrm{s}$. The mass units are determined by the activity flag, Menu 1.3. If the mass units are curies, the DUST-MS code internally transforms this into grams to maintain consistency. The main menu for Source/Sink parameters follows.

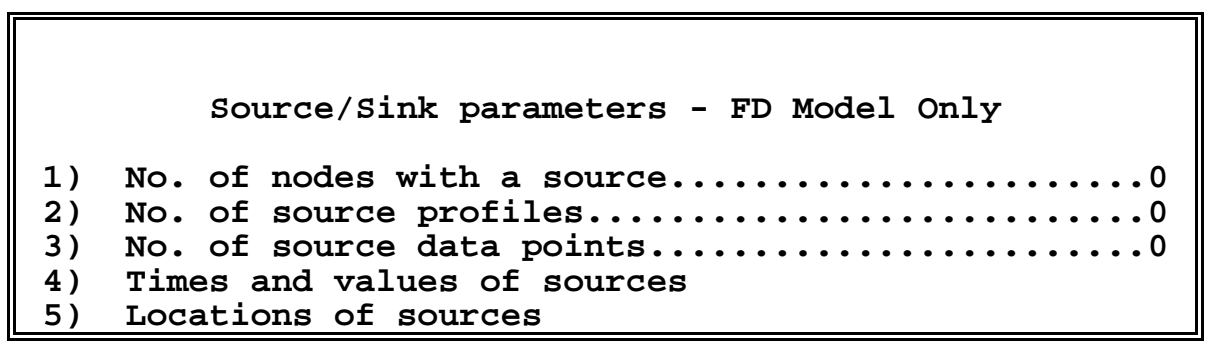


6) Source profile assignment to nodes with source

7) Exit to MAIN MENU

If there are no external sources, items 4 - 6 of the menu are not used by the DUST-MS code. DUSTINMS prevents the user from inputting values for these items when the number of source nodes is zero.

\subsubsection{Number of Source Nodes}

The total number of locations where a source exists ranges up to 500, however, it is not to exceed the number of nodal (control volume) points specified in Menu 1.4.

\subsubsection{Number of Source Profiles}

The DUST-MS code accepts up to 8 different source profiles. This value must be non-zero if the number of source nodes is non-zero.

\subsubsection{Number of Source Data Points}

Each source profile table must contain at least two points and no more than 8 points. The specified number of data points applies to every profile.

\subsubsection{Times and Values of the Sources}

The magnitude of the source is specified in a table which contains a set of ordered pairs of time (years) and source strength (mass $/ \mathrm{cm}^{3} / \mathrm{s}$ ). The code prompts the user for the ordered pairs to be entered on a single line.

In the following example, the user previously requested 2 source profiles with 4 data points. The code prints the current values for the table and then asks if further changes are requested. In this case, profile number 1 has a concentration of $1 \mathrm{E}-5 \mathrm{Ci} / \mathrm{cm}^{3} / \mathrm{s}$ between 0 and 10 years. This decreases to zero at 10.01 years and remains there until 1000 years. Interpolation is used to determine the calculation at times not in the table. Therefore, the first time should be the problem start time (normally 0) and the last time should be greater than the maximum problem time, Menu 2.7. 


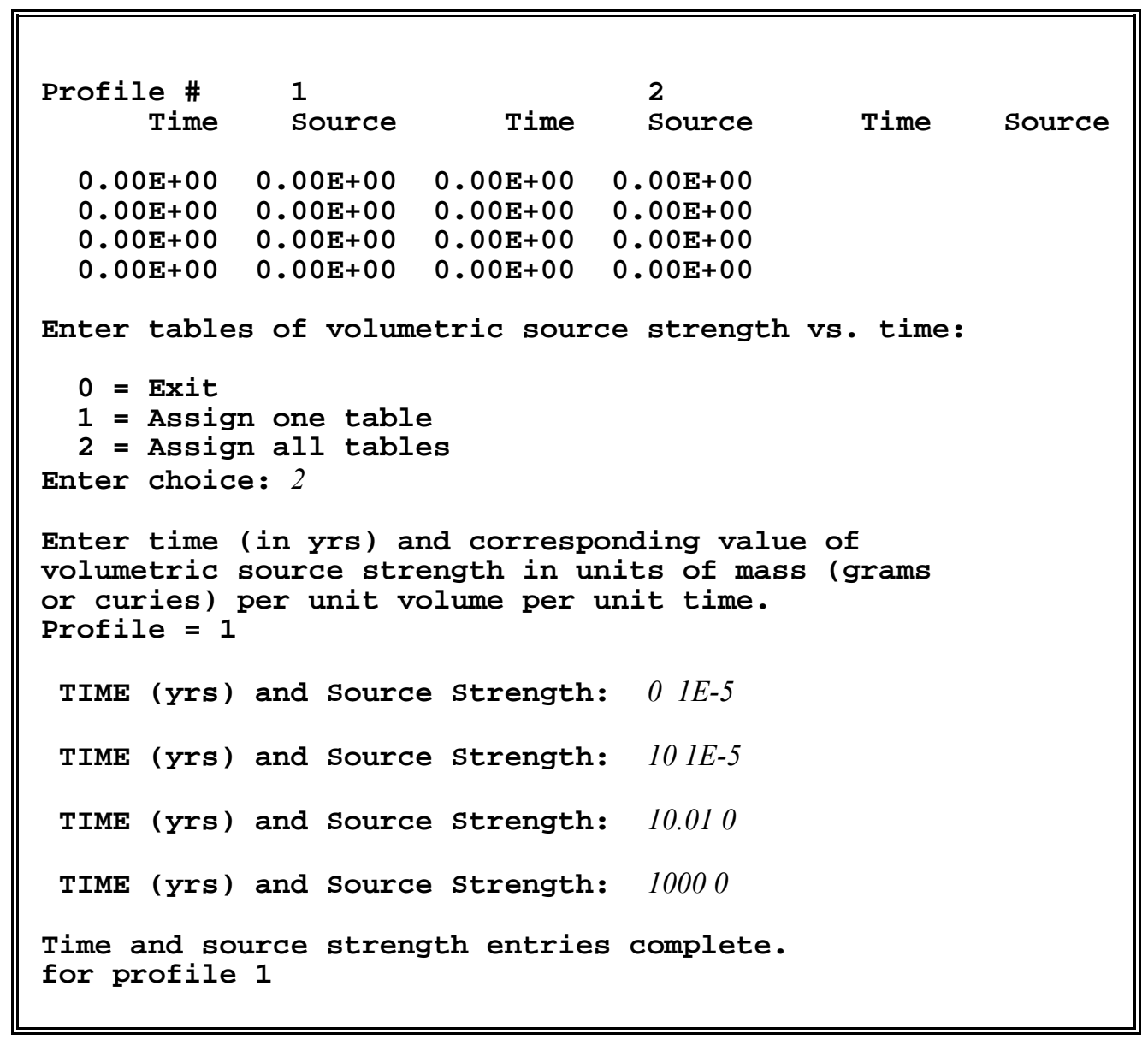

At this point, the code would prompt the user for the second source profile. Upon completion of this input, the current values are printed for review.

\subsubsection{Source Locations}

Each source must be assigned to a specific nodal location within the modeled domain. In the following example, the user requested only two source points. These are specified as occurring in Node 30 and 40. 


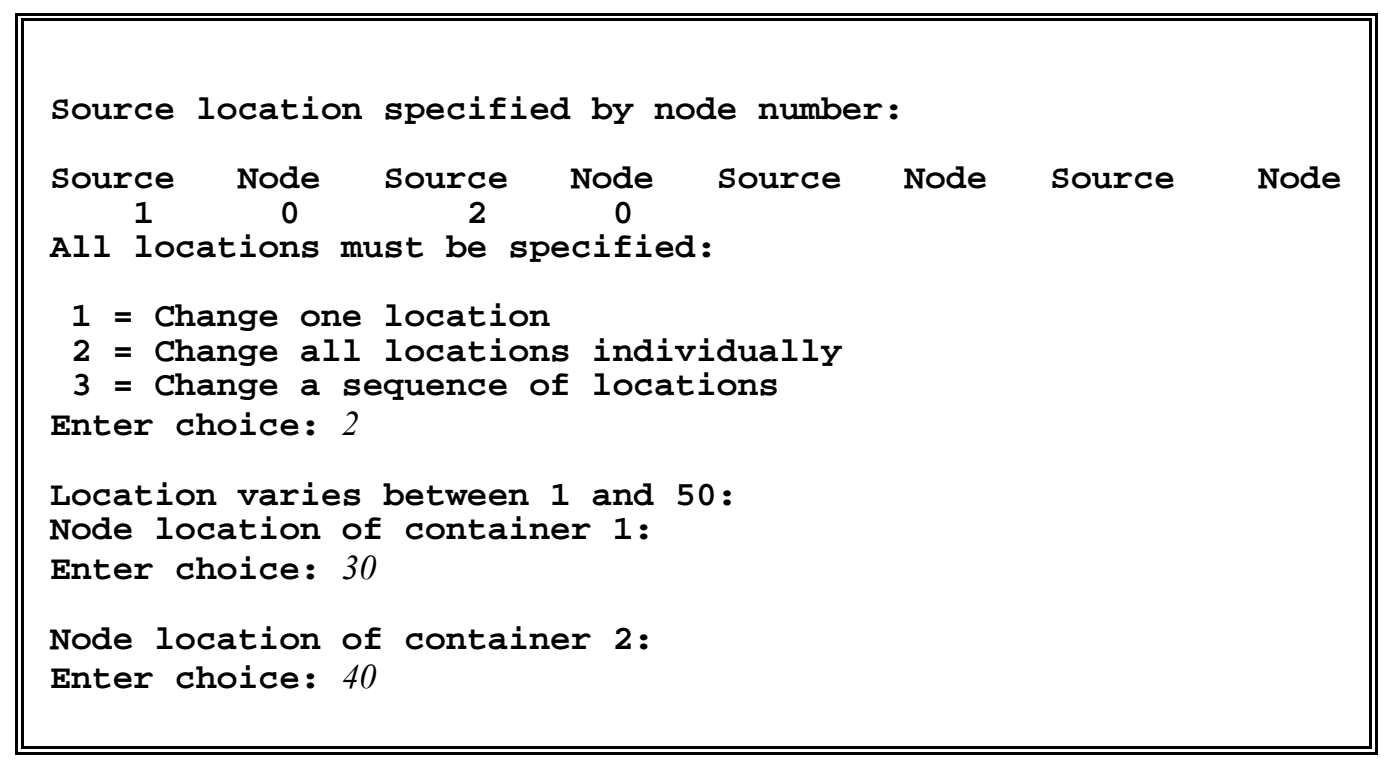

\subsubsection{Source Type Assignments}

Each of the different source nodes must be assigned a source type. In this example, there are two source nodes and two source types. Source type 1 is assigned to source 1 in node 30 . Source type 2 is assigned to source 2 .

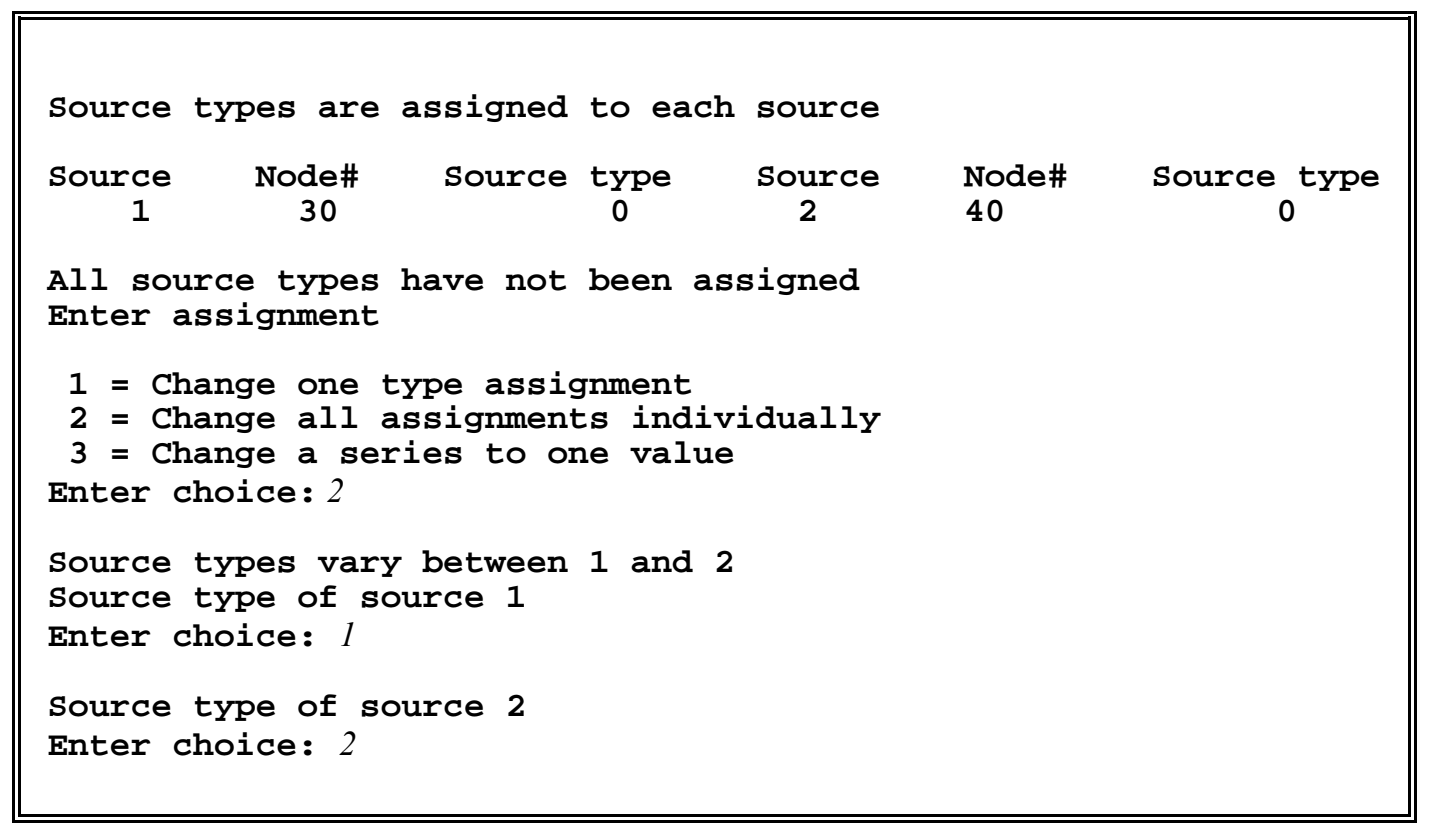




\subsection{Create an Input Deck for Use by the DUST-MS Code}

After specifying all of the input variables, the user may attempt to create a DUST-MS input deck. Upon entering this menu, the code prompts the user for the file name to give to the input deck. This file name may include the path. If the path is omitted, the file is stored in the current directory. The user is also asked if an attempt should be made to read the input file using the routine found in the DUST-MS code. If this is not done, or the input deck is valid, control is returned to the main menu. If an attempt is made to read an input deck that is not valid, execution of DUSTINMS is terminated and control is returned to the operating system. A typical input session follows.

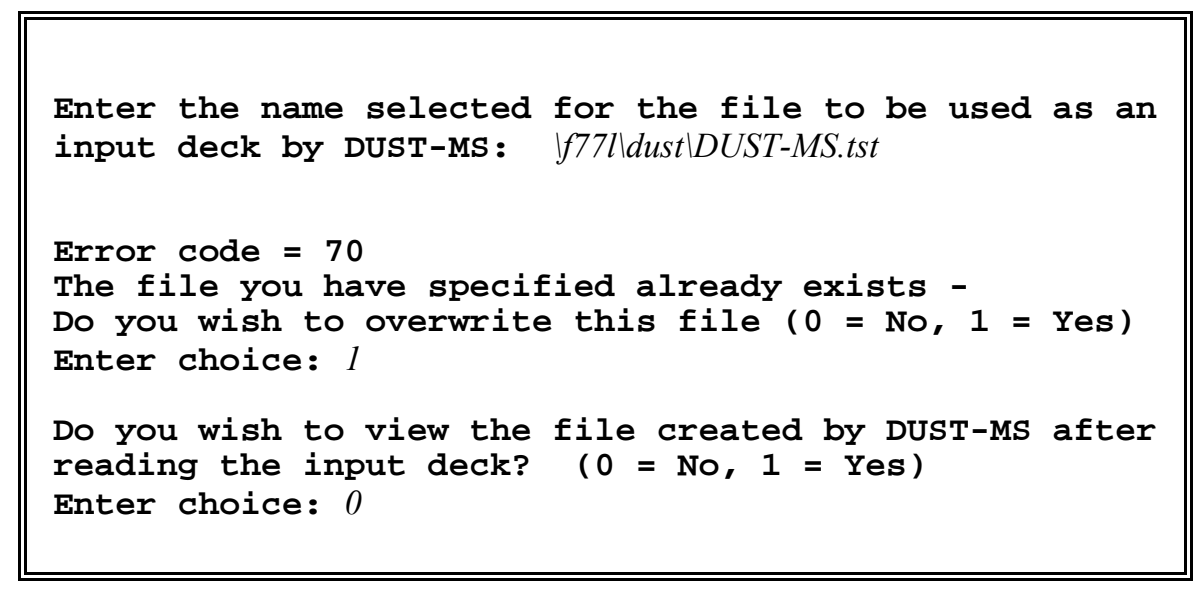

If the input file created by this process is not valid, attempts to use the DUST-MS code will cause the program to "bomb." Also, attempts to read this file back in to DUSTINMS will cause DUSTINMS to "bomb" because the input routines are identical. For this reason, it is strongly recommended to create a BLOKXXX file prior to creating an input deck.

\subsection{Store Partial Input in a BLOKXXX File}

If the code user has not completed the process of creating an input deck, or if a backup copy of all specified input is desired, a BLOKXXX file may be created. All input variables required by the DUST-MS code are part of a NAMELIST. This NAMELIST is written to the BLOKXXX file when using this routine. The XXX in the file name is a three-digit indicator that can be used to uniquely identify separate files.

A typical input routine using this menu follows. If the file exists, the code asks the user if the file is to be overwritten. 


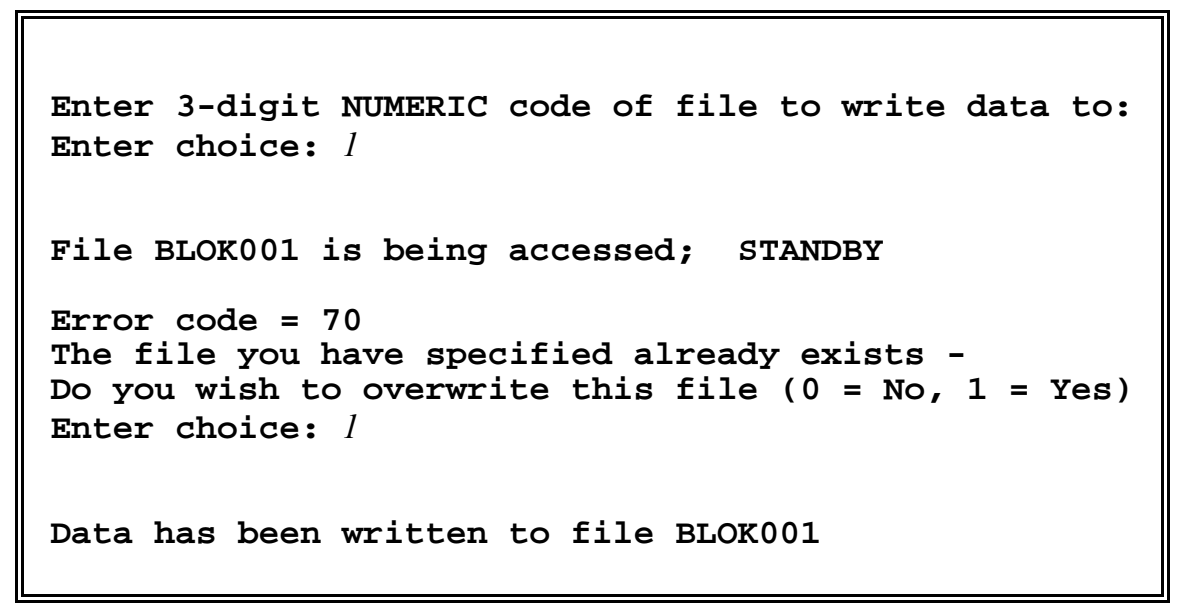

\subsection{Exit the Program}

This selection exits the program without saving data and returns control to the operating system. 


\section{STRUCTURE OF A DUST-MS and DUSTMS-D INPUT DECK}

A DUST-MS input deck is composed of a series of Data Sets which are groupings of data covering a particular topic. There are 10 Data Sets as follows:

1) General problem definition;

2) Time parameters;

3) Material assignments/properties;

4) OUTPUT specifications;

5) Facility dimensions and coordinates;

6) Initial and boundary conditions;

7) Water flow and moisture content;

8) Container parameters;

9) Wasteform parameters; and

10) Source/Sink parameters.

Information is required for each Data Set. However, the user does not need to invoke all of the models during a simulation. For example, if wasteforms are not modeled, Data Set 8 would contain one data input line telling the code that the number of containers is zero and Data Set 9 would contain a similar line indicating that there are no wasteforms. Data Set 10 has non-zero values only when external source/sink (i.e., not wasteform sources) are used.

Within a DUSTMS-D input deck, there are two major categories of input lines, label lines and data input lines (for notational convenience, each line of the input deck is referred to as a line). The beginning of each Data Set within DUSTMS-D is denoted with a label line. It is recommedned that the label line contain the name of the Data Set and a brief description of its contents, e.g., "Data Set 8 - Container Parameters." The label can be any alpha-numeric string with a length of 79 characters or less. For example, the user may want to put more information on the identifier, e.g., "DS-8 - Container Parameters - Gaussian Failure " would be an acceptable label. Following the Data Set label line are the data input lines. In the data input lines, the first 10 columns on each line are reserved for comments supplied the code user. The comments are used to identify the variables contained on the line and can be any alpha-numeric string of up to 10 characters. For example, for the container parameter TIMEB, which gives the time of general failure of the container, the comment included could be "TIME FAIL." All data sets are separated by a label line which is typically left blank.

Label lines are also used to identify the major property input parameters which are input several to a line. These include parameters describing transport in soil, container degradation, and wasteform release. These label lines will be discussed in the appropriate sections of this chapter.

A typical input deck is displayed in Table 7.1. 
The modeled problem has 200 finite difference control volume. There is only one material type. There are three isotopes being simulated, Th-230, Ra-226, Rn-222. There are 20 containers, the first ten fail after 50 years, the remainder after 100 years. There is one waste form type. Examples of input requirements for distributed container failure models are provided and discussed in the container corrosion parameters, Data Set 8, section.

The remainder of this chapter provides the structure of the DUSTMS-D input deck. All input is read into the code through formatted read statements. For clarity, each of the formatted read statements will be supplied along with the FORMAT statement. These are supplied in the single line boxes in this chapter. Often at the top of the box, a few lines in italics will appear. These are the actual input lines found in Table 7.1 A blank line is often used to signal the end of a data set. These are indicated in the examples of this chapter by the words "BLANK LINE."

The code DUSTMS-D makes extensive use of the subroutines INPTR, INPTI, INPTR2, READR, and READN for reading the input data. These procedures are explained below and will be referred to later in this chapter as necessary.

Table 7.1 Example DUSTMS-D Input deck

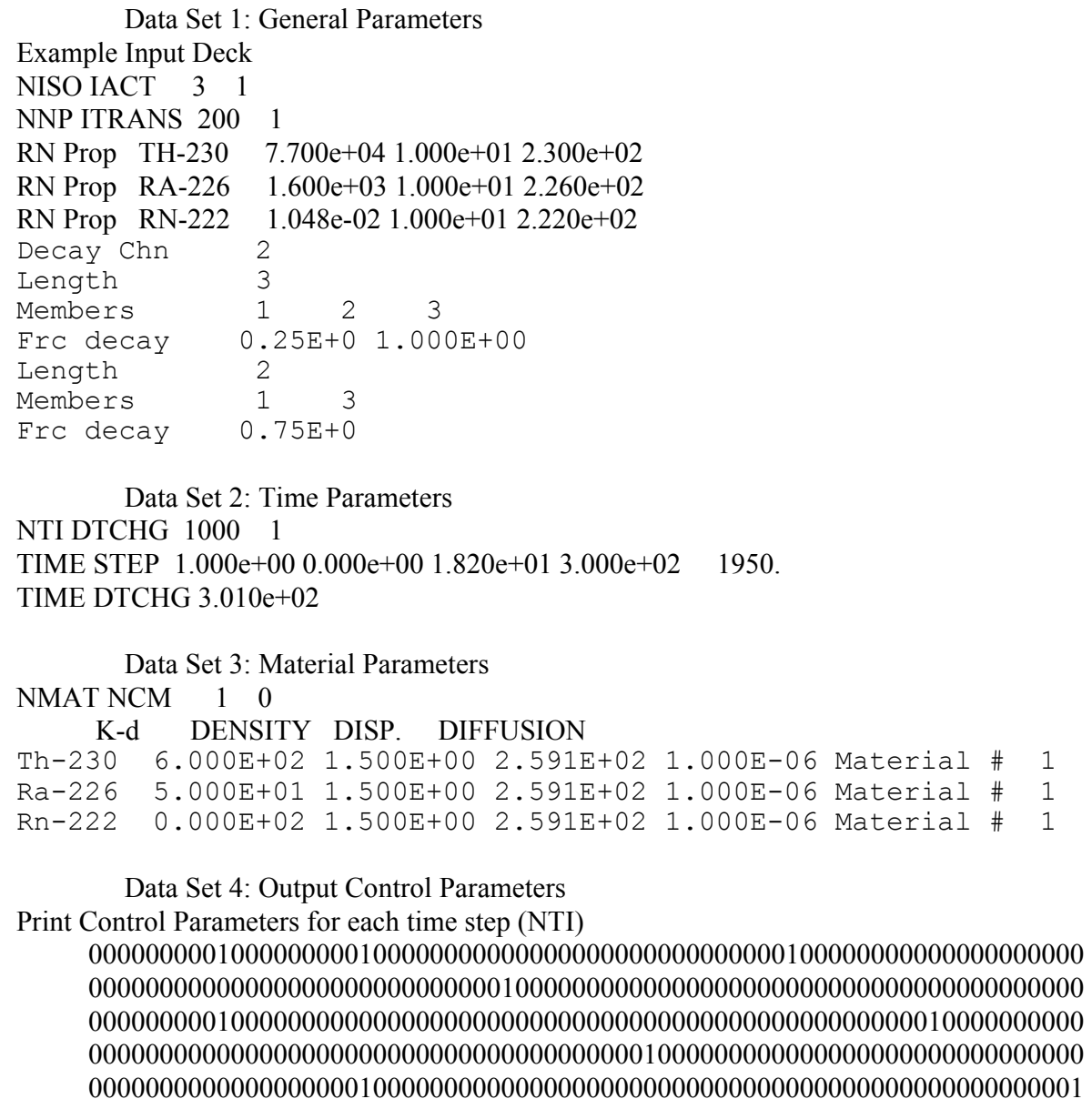


0000000000000000000000000000000000000000000000000100000000000000000000 0000000000000000000000000000010000000000000000000000000000000000000000 0000000001000000000000000000000000000000000000000000000000010000000000 0000000000000000000000000000000000000001000000000000000000000000000000 0000000000000000000100000000000000000000000000000000000000000000000001 0000000000000000000000000000000000000000000000000100000000000000000000 0000000000000000000000000000010000000000000000000000000000000000000000 0000000001000000000000000000000000000000000000000000000000010000000000 0000000000000000000000000000000000000001000000000000000000000000000000 00000000000000000001

Trace var $\begin{array}{lllll}6 & 6 & 3 & 5\end{array}$

Con Tr Loc $10 \quad 20 \quad 30 \quad 40 \quad 50 \quad 60$

FX Tr Loc $10 \quad 20 \quad 40 \quad 60 \quad 80 \quad 90$

\begin{tabular}{lccccccc}
\multicolumn{8}{c}{ Data Set 5: Facility Coordinate Data } \\
Area & $1.000 \mathrm{E}+08$ \\
Delta-X & 1 & 5 & 1 & $0.000 \mathrm{E}+00$ & $5.000 \mathrm{E}+01$ & $0.000 \mathrm{E}+00$ \\
Delta-X & 6 & 195 & 1 & $2.500 \mathrm{E}+02$ & $5,000 \mathrm{E}+01$ & $0.000 \mathrm{E}+00$ \\
& 0 & 0 & 0 & & 0 & 0 & 0
\end{tabular}

Data Set 6: Initial and Boundary Conditions

TH-230 INV $\quad \begin{array}{llllll}2 & 200 & 1 & 0.000 \mathrm{E}+00 & 0.000 \mathrm{E}+00\end{array}$

$\begin{array}{llllll}0 & 0 & 0 & 0 & 0 & 0\end{array}$

TH-230 INV $\quad \begin{array}{lllllll}1 & 200 & 1 & 0.000 \mathrm{E}+00 & 0.000 \mathrm{E}+00\end{array}$

$\begin{array}{llllll}0 & 0 & 0 & 0 & 0 & 0\end{array}$

TH-230 INV $\quad \begin{array}{lllllll}1 & 200 & 1 & 0.000 \mathrm{E}+00 & 0.000 \mathrm{E}+00\end{array}$

$\begin{array}{llllll}0 & 0 & 0 & 0 & 0 & 0\end{array}$

$\begin{array}{lllll}\text { BC FLAGS } & 2 & 1 & 7 & \mathrm{TH}-230\end{array}$

Time Top 0.000E+00 1.000E+02 2.000E+02 3.000E+02 4.000E+02 6.010E+02 8.010E+02

$\begin{array}{lllllllll}\text { VALUE } & 0.000 \mathrm{E}+00 & 0.000 \mathrm{E}+00 & 0.000 \mathrm{E}+00 & 0.000 \mathrm{E}+00 & 0.000 \mathrm{E}+00 & 0.000 \mathrm{E}+00 & 0.000 \mathrm{E}+00\end{array}$

Time Bot $\quad 0.000 \mathrm{E}+00 \quad 1.000 \mathrm{E}+02 \quad 2.000 \mathrm{E}+00 \quad 3.000 \mathrm{E}+02 \quad 4.000 \mathrm{E}+02 \quad 6.010 \mathrm{E}+02 \quad 8.010 \mathrm{E}+02$

$\begin{array}{llllllll}\text { VALUE } & 0.000 \mathrm{E}+00 & 0.000 \mathrm{E}+00 & 0.000 \mathrm{E}+00 & 0.000 \mathrm{E}+00 & 0.000 \mathrm{E}+00 & 0.000 \mathrm{E}+00 & 0.000 \mathrm{E}+00\end{array}$

$\begin{array}{lllll}\text { BC FLAGS } & 2 & 1 & 2 & \mathrm{RA}-226\end{array}$

TIME TOP $0.000 \mathrm{E}+003.000 \mathrm{E}+02$

VALUE $\quad 0.000 \mathrm{E}+000.000 \mathrm{E}+00$

TIME BOT $\quad 0.000 \mathrm{E}+003.000 \mathrm{E}+02$

VALUE $\quad 0.000 \mathrm{E}+00 \quad 0.000 \mathrm{E}+00$

$\begin{array}{lllll}\text { BC FLAGS } & 2 & 1 & 2 & \mathrm{RN}-222\end{array}$

TIME TOP $0.000 \mathrm{E}+003.000 \mathrm{E}+02$

VALUE $\quad 0.000 \mathrm{E}+00 \quad 0.000 \mathrm{E}+00$

TIME BOT $\quad 0.000 \mathrm{E}+00 \quad 3.000 \mathrm{E}+02$

VALUE $\quad 0.000 \mathrm{E}+000.000 \mathrm{E}+00$

Vel Pts 2

TIME $\quad 0.000 \mathrm{e}+003.000 \mathrm{e}+02$

VALUE 2.316e-04 2.316e-04

$\begin{array}{lllllll}\text { MST CONT } & 1 & 200 & 1 & 1.000 \mathrm{E}-01 & 0.000 \mathrm{E}+00\end{array}$

$\begin{array}{llllll}0 & 0 & 0 & 0 & 0 & 0\end{array}$

Data Set 8: Container Parameters

NCON TYPE $20 \quad 1 \quad 0$

TIME FAIL 5.000E+01 5.000E+01 5.000E+01 5.000E+01 5.000E+01 5.000E+01 5.000E+01

TIME FAIL 5.000E+02 5.000E+02 5.000E+02 $1.000 E+02 \quad 1.000 E+02 \quad 1.000 E+02 \quad 1.000 E+02$

TIME FAIL 1.000E+02 1.000E+02 1.000E+02 1.000E+02 1.000E+02 1.000E+02

$\begin{array}{lllllll}\text { Time Bur } & 1950 . & 1952 . & 1955 . & 1961 . & 1965 . & 1968 .\end{array}$ 


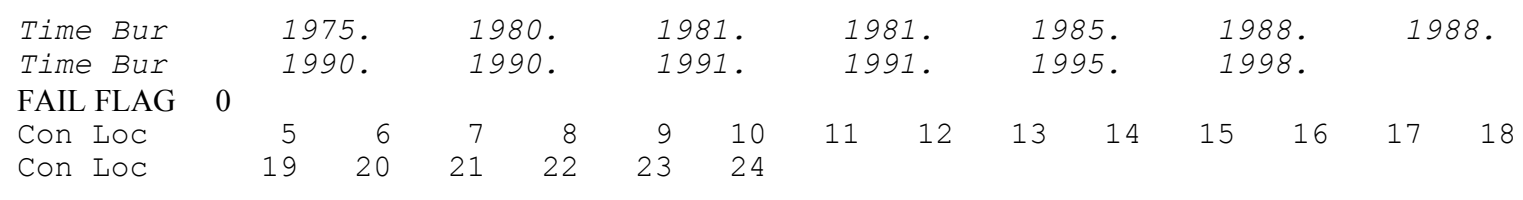

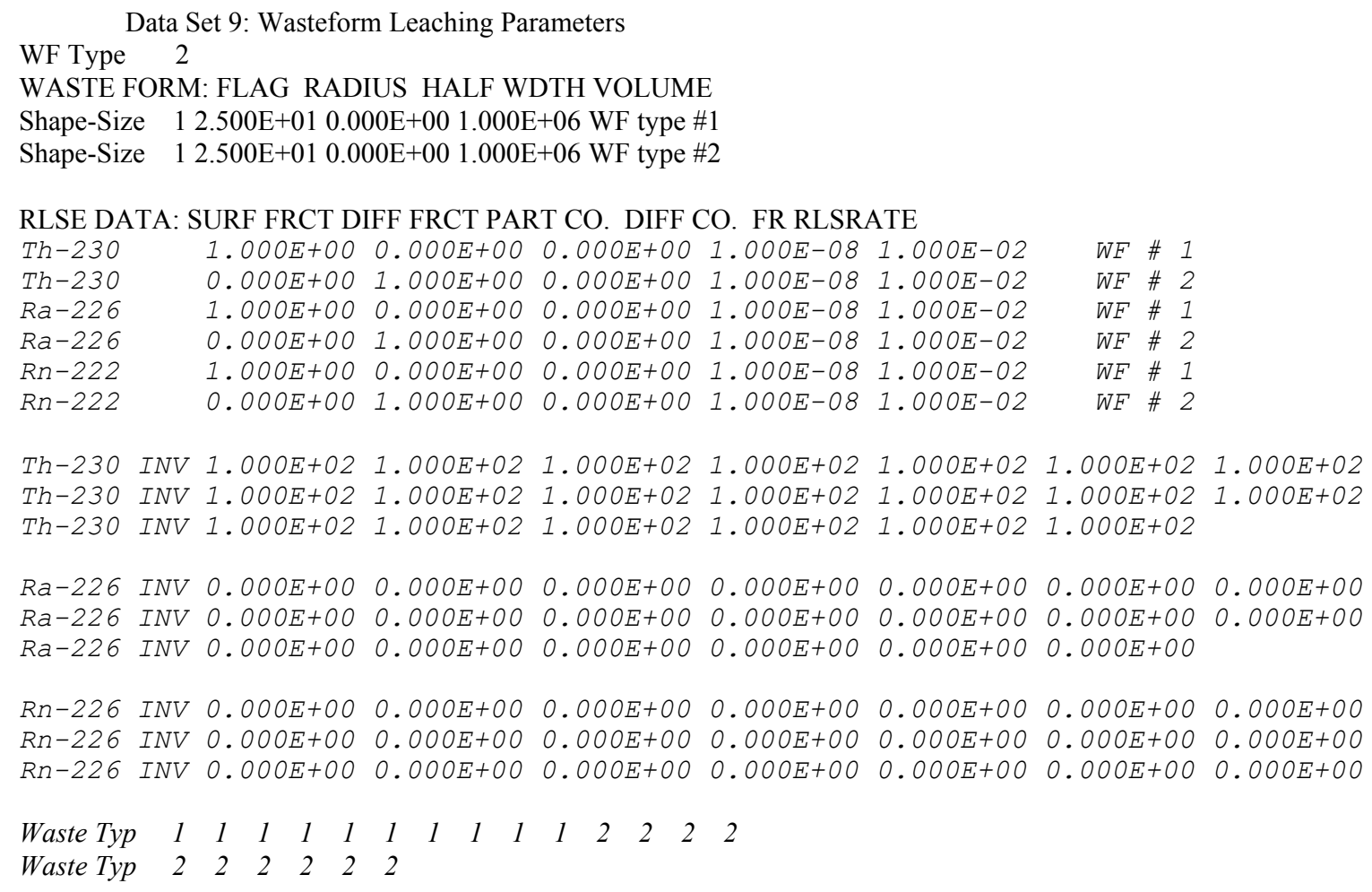

Data Set 10: External Sources (F.D. model only)

TH-230 SRC 0000

RA-226 SRC 0000

RN-222 SRC $\quad 0 \quad 0 \quad 0$

\section{$\underline{\text { Subroutine INPTR }}$}

The DUSTMS-D code contains a subroutine, INPTR, which reads in real variables in blocks of 7 with a label in columns 1 - 10. The formatted read statement in INPTR is:

\section{READ(5,20) SL,(F(I),I=IMN,IMX) \\ 20 FORMAT(A10,7D10.0)}


The variables, IMN and IMX are calculated by the code. In INPTR, there is a DO WHILE loop which increases the value of IMN until it exceeds the maximum number of input values requested. For example, the first time through the loop, IMN $=1$ and $I M X=7$. The second time through the loop, IMN $=8$ and IMX $=14$, and so on. The value for IMX is limited to be no greater than the maximum number of variables to be specified. For example, if the maximum number to be specified is 10 , the second time through the loop IMX $=10$. In attempting to go through the loop a third time, IMN would be 15 which is greater than the maximum. Therefore, the input routine is terminated.

The array $\mathrm{F}$ and the maximum number of variables to be specified are determined through arguments in the call statement.

Subroutine INPTR is used to define the variables FBRANCH (branching fractions for decay reactions), TIMOUT (times at which the time step is reset in the FD model), TIMEF (time of container failure), and WTINIT (initial mass in each container).

\section{$\underline{\text { Subroutine INPTI }}$}

Subroutine INPTI is the analogue of INPTR for integer numbers. In this subroutine, 14 integer values are input per line. The read statement is:

\section{READ(5,10) SL,(NF(I),I=IMN,IMX) \\ 10 FORMAT(A10,14I5)}

The first time through, IMN is 1 and IMX is the minimum of 14 or the maximum number to be redefined. Again, these values are increased in steps of 14 until IMN exceeds the maximum value. arguments.

The array NF and the maximum number of input variables are defined through calling

Subroutine INPTI is used to define ICHAIN (pointer array that defines the decay chain), NTRCEC (nodes for concentration traces), NTRCEF (nodes for flux traces), IPIT (flag for modeling local failure), NELCON (nodal location of all containers), ICTYPE (container type at each location), IWTYPE (waste type at each location), LSRC (nodal location of external sources), and ISTYPE (source type at each location).

$\underline{\text { Subroutine INPTR2 }}$ 
Subroutine INPTR2 is identical to INPTR except that instead of defining a one-dimensional array, a two-dimensional array is defined. Two dimensional arrays are used to specify all of the tables involving a variable as a function of time. The read statement is:

\section{READ(5,20) SL,(F(I,J),I=IMN,IMX)) \\ 20 FORMAT(A10,7D10.0)}

The procedure used to define IMN and IMX is identical to that used in INPTR. The dummy array $\mathrm{F}$, the maximum number of values to be defined, and the index $\mathrm{J}$ are all defined in the calling arguments. Typically, the call statement for INPTR2 is embedded in a loop in which J increases from 1 to the number of profiles requiring input.

Subroutine INPTR2 is used to define the tables of boundary condition versus time at both boundaries, Darcy velocity versus time, and source strength versus time.

\section{$\underline{\text { Subroutine READR }}$}

READR takes a set of six numeric values and calculates a series of coordinates based on these values. These values are:

1) Number of the first node in the sequence, $\mathrm{NI}$

2) Number of nodes defined by the sequence, NSEQ

3) Number to add to each node in the sequence, NAD

4) Value for the first node in the sequence, FNI

5) Value to add to each node in the sequence, FAD

6) Fractional increment in value for each node in the sequence, FRD

The location is specified through the following equation:

$$
\mathrm{F}(\mathrm{NI}+\mathrm{I} * \mathrm{NAD})=\mathrm{FNI}+\mathrm{FAD} *(1+\mathrm{FRD})^{* *}(\mathrm{I})
$$

In the example presented in 7.1, the user specified the location of all 200 nodes to be $50 \mathrm{~m}$ apart. With the following lines:

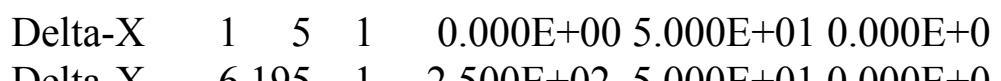

$\begin{array}{llllll}\text { Delta-X } & 6195 & 1 & 2.500 \mathrm{E}+02 & 5.000 \mathrm{E}+01 & 0.000 \mathrm{E}+0\end{array}$ 
Although, this example uses constant mesh spacing, it illustrates the process needed to define nonconstant mesh spacing using multiple lines of input.

The format for this input statement in subroutine READR is:

\section{READ(5,40) SL,NI,NSEQ,NAD,FNI,FAD,FRD \\ 40 FORMAT(A10,3I5,5X,3(D10.3))}

The subroutine READR continues to read input until the value for NI is zero. Therefore, a blank line is often used to signify the end of all READR input variables. After a zero is input, the code checks to insure that all NNP locations are defined (NNP is the number of nodal points specified in Menu 1). READR is also used for input of initial concentration and moisture content at each location.

$\underline{\text { Subroutine READN }}$

READN is only used when redefining the location at which certain material properties exist. For this reason, the discussion of READN can be found in Section 7.3.

\subsection{DATA SET 1: Title and General Problem Definition (Menu 1)}

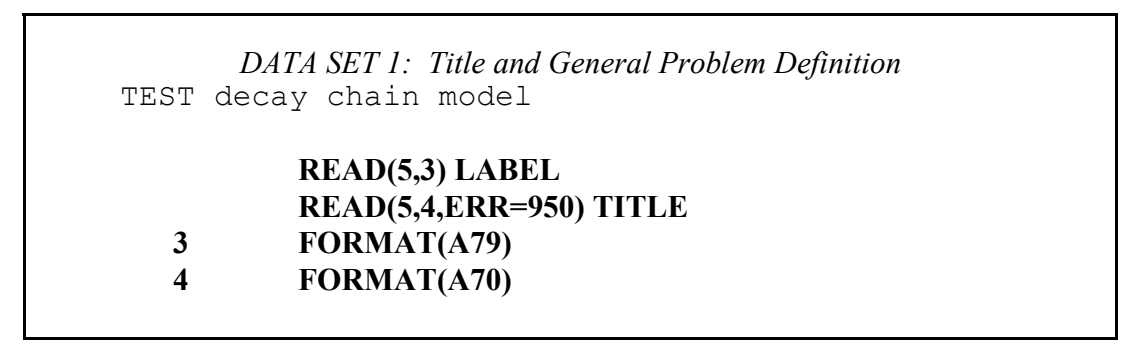

The first line in the input deck is the label for Data Set 1. The second line contains the title for this problem and can be up to 70 characters in length. The format for reading the LABEL remains unchanged in DUSTMS-D and will not be repeated in the remainder of this chapter.

The general problem definition includes the number of isotopes in the simulation, the mass activity flag, the number of nodal points, radionuclide specific data, and information defining the decay chains. The first line contains the number of isotopes and the mass activity flag $(0=$ mass input in grams, 1 = mass input in Curies, $2=$ mas input in Becquerels). The second line contains the number of nodal points in the simulation. 
The next set of lines contains information to define the radionuclide specific properties (name, half-life in years, solubility limit $\left(\mathrm{gm} / \mathrm{cm}^{3}\right.$ ), and atomic mass (used in converting from Curies or Becquerels to grams)). There is one line for each isotope in the simulation (three in this example)..

The final set of lines defines the decay chains that are simulated. The first line in this set reads the number of decay chains. If this value is zero, no further lines are required in this data set. For non-zero values, input is required to define the decay chains. The example has two decay chains, for each decay chain, three lines of input are required. The first provides the number of members (length) in the decay chain, the second defines the order of the decay chain, and the third defines the fractional decay to the progeny (branching fraction, useful when an isotope decays directly to more than one progeny).

In this example, the first chain has three members. The members of the chain are isotopes 1 , 2 , and 3 where the isotope number corresponds to the order in which the radionuclides are input. In this example, isotope 1 is Th-230, isotope 2 is Ra-226, and isotope 3 is $\mathrm{Rn}-222$. The next line of input defines the branching fractions. In this hypothetical example, the fraction of Th-230 that decays to Ra-226 0.25. The fraction of Ra-226 that decays to Rn-222 is 1.0 . Note, the number of branching fractions required is one less than the length of the chain. The second chain has two members and Th-230 decays to Rn-222 with a branching fraction of 0.75 . Due to difficulties in calculating ingrowth for distributed failure models, it is not recommended to have a radionuclide receiving ingrowth in more than one decay chains. Receiving ingrowth from multiple radionuclides has not been tested for the distributed failure models.

\begin{tabular}{|c|c|}
\hline $\begin{array}{l}\text { NISO IACT } \\
\text { NNP ITRANS }\end{array}$ & 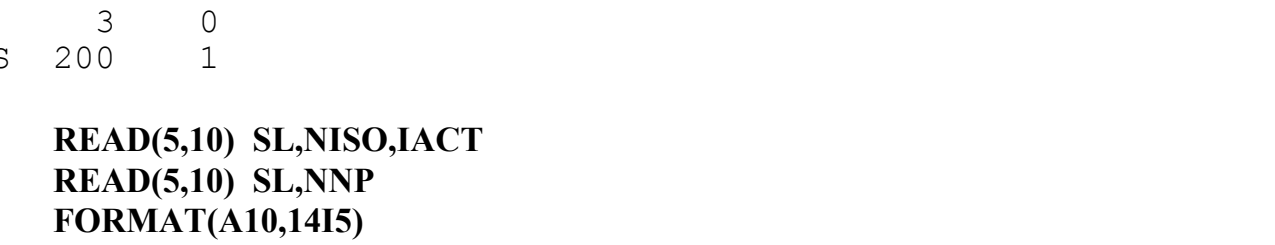 \\
\hline $\begin{array}{ll}\text { RN } & \text { Prop } \\
\text { RN } & \text { Prop } \\
\text { RN } & \text { Prop }\end{array}$ & 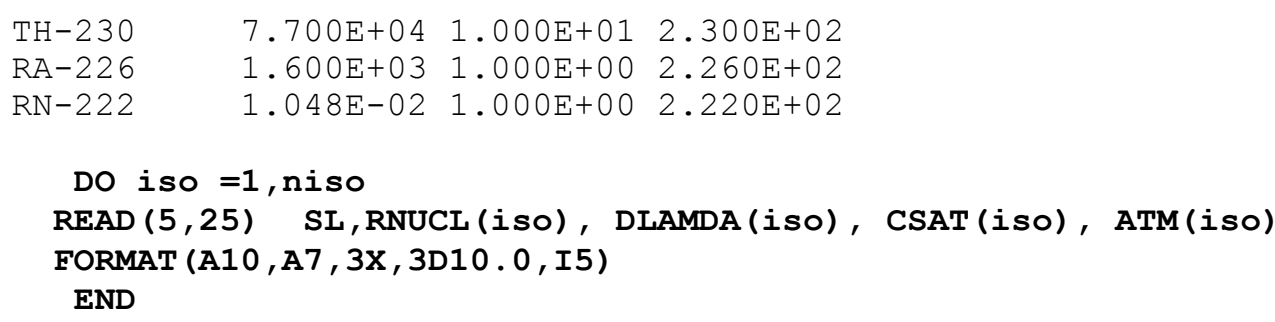 \\
\hline $\begin{array}{l}\text { Decay Chn } \\
\text { Length } \\
\text { Members }\end{array}$ & $\begin{array}{l}2 \\
3 \\
1\end{array}$ \\
\hline
\end{tabular}




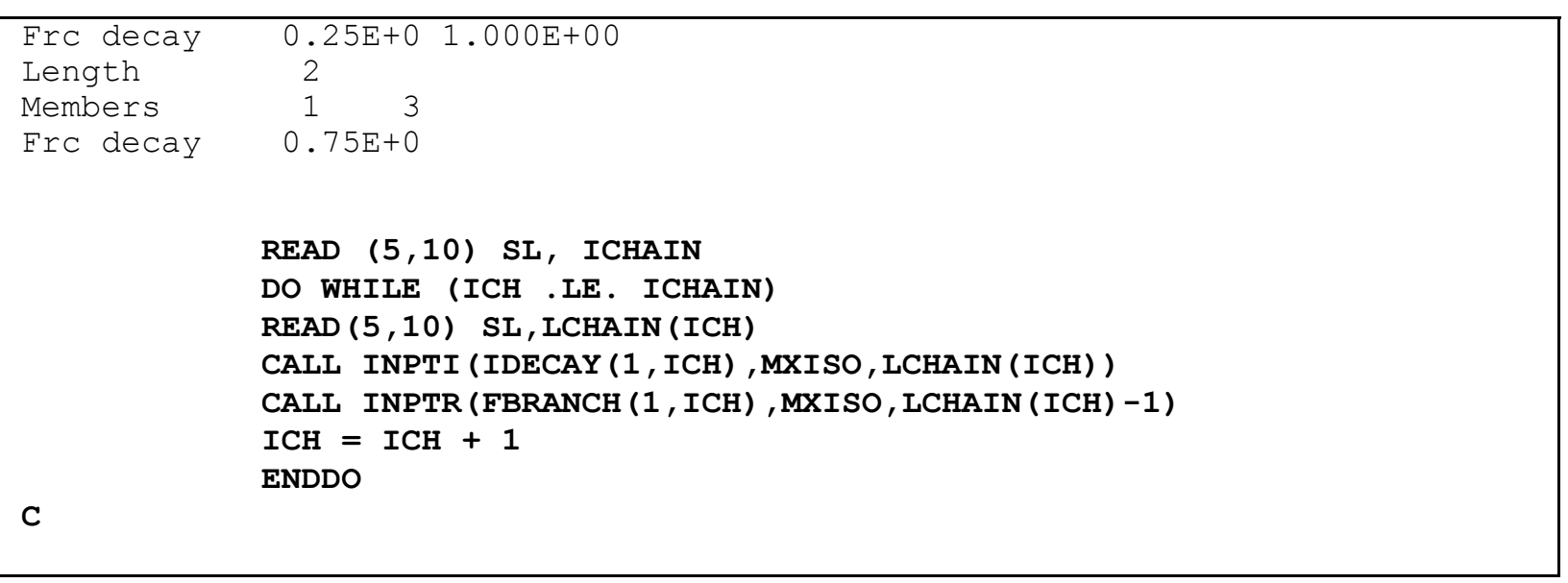

The parameter SL appears at the beginning of each data input line and is the 10 character label used to identify the data on the line. The FORMAT identified by statement 10 is used to read in all integer parameters used within DUSTMS-D. The variables LABEL and SL are not used by the DUSTMS-D code. Their sole purpose is to identify variables in the input deck.

\subsection{DATA SET 2: Time Parameters (Menu 2)}

This data set includes all the parameters that set time step size and simulation time. The first line contains the maximum number of time steps used and the variable NDTCHG. NDTCHG represents the number of times at which the time step is to be reset to its original value

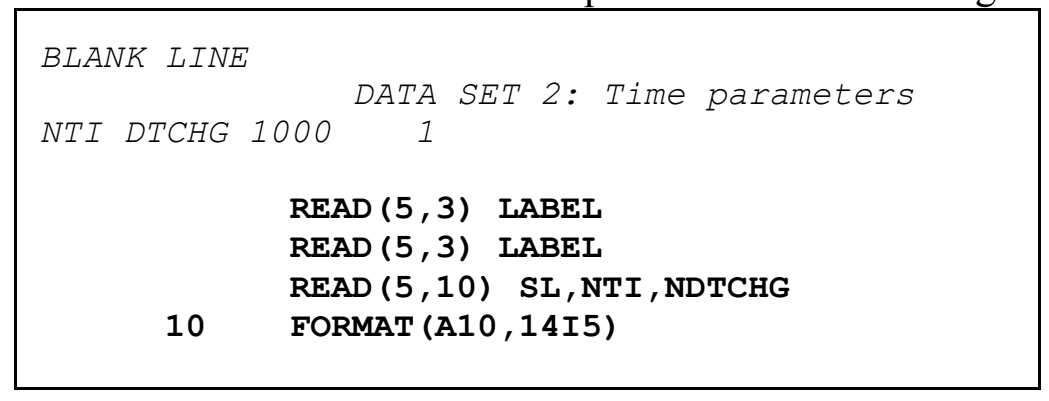

The following input line which specifies the time step parameters, (initial time step, fractional change in time step, maximum time step, and maximum simulation time) is required. All times in the DUSTMS-D code are input in units of years. The maximum number of time steps is 10,000 .

TIME STEP 1.000E+00 0.000E-00 1.82000E+01 3.00E+02 1950.

READ (5,20) SL,DELT,CHNG,DELMAX,TMAX,TIMSTRT 
In the example problem, Table 7.1, the initial time step is 1.0 years while the maximum time step is 18.2 The selection of the variable $\mathrm{CHNG}=0$ makes all time steps equal to the initial time step of one year. The maximum simulation time is 300 years. The problem start time is 1950 . This start time is used to calculate decay for wastes emplaced at different times. The emplacement time is discussed in Data Set 8.

The final set of data lines in this set specifies NDTCHG. If NDTCHG $=0$ this line is not required. DUST uses the input routine INPTR to read in the values. The formatted read is:

TIME DTCHG 3.010E+02

20

READ (5,20) SL,(F(I),I=IMN,IMX)

FORMAT(A10,7D10.0)

On this call to INPTR, the dummy array F represents the array TIMOUT. As on all calls to INPTR, an input line is required for every 7 values of this variable.

\subsection{DATA SET 3: Material Assignments/Properties (Menu 3)}

As with each new Data Set, the first two lines are label lines indicating the end of the previous data set and the beginning of a new data set. The input statements are:

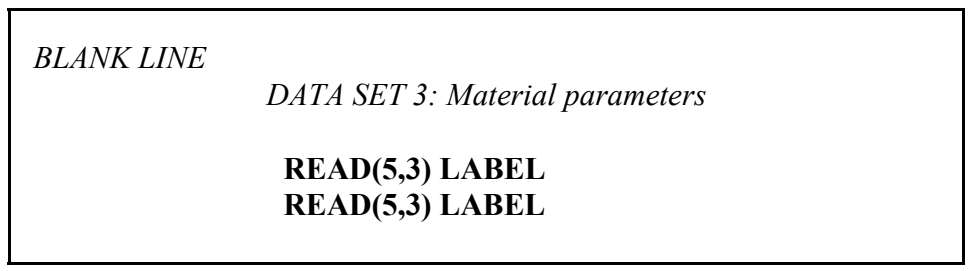

The DUSTMS-D model allows up to 10 material types and four material properties.

The first line required defines the number of materials (NMAT) and the number of materials that will be redefined from material $1(\mathrm{NCM})$. Initially all nodes are assumed to have material properties specified by material 1 , the default material.

NMAT NCM 10 


\section{0 \\ READ(5,10) SL,NMAT, NCM \\ FORMAT(A10,14I5)}

The next group of lines in this set requires the material properties for each material. All material properties are isotope specific. The model has NMPPM properties (NMPPM $=4)$. The properties for material J are PROP(1,J,ISO), the distribution coefficient; PROP(2,J,ISO), the soil bulk density; PROP(3,J,ISO), the dispersivity; and PROP(4,J,ISO), the effective diffusion coefficient (tortuosity effects should be included in this parameter). This set of lines requires a label line. The label line, if written by DUSTINMS, contains identifiers for the four material properties. The input routine requires all properties for a material and a given isotope to appear on a single line. Note, DUSTINMS automatically wrote an identifier after the last variable (diffusion coeffiecient). This information is not read by DUSTMS-D and is supplied to inform the code user. The input procedure is repeated for each material as follows:

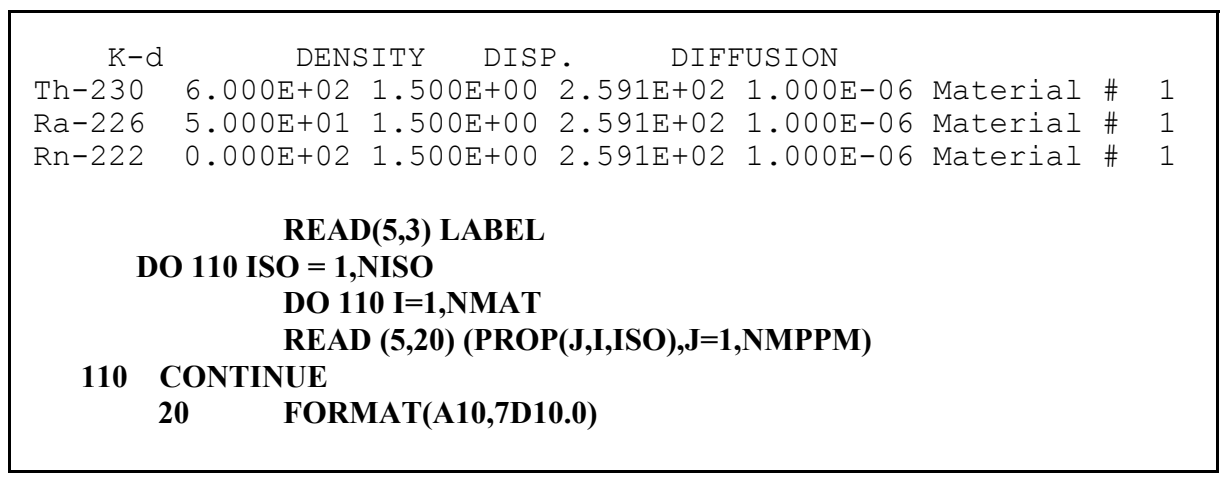

If more than one material is present, the data structure requires that material properties for each isotope are input prior to collecting input for the next isotope in the order.

The final set of lines define the link between nodes and material type. Material type assignment is achieved using the subroutine READN. If the number of changed materials is zero, or the number of material types is 1, this set of lines is not required, as in Table 7.1.

READN reads a sequence of lines having five integer values per line. The five values are used to automatically generate the material type reassignment. For material type assignment, the five variables on each line are:

a) the location (node number) of the first node in the sequence (NI),

b) the number of nodes to be assigned (NSEQ),

c) the increment of nodes in the sequence (NADD),

d) the material type for the first node in the sequence (NITYP), and

e) the increment of material type for each node in the sequence (NTYPAD). 
READN uses the following equation to assign material types

$$
\text { IMAT(NI+NADD*I) }=\text { NITYP }+ \text { NTYPAD*I }
$$

where I ranges from 0 to NSEQ-1. The read statement is:

$$
\begin{array}{ll} 
& \text { READ(5,30) SL,NI,NSEQ,NAD,NITYP,NTYPAD } \\
30 & \text { FORMAT(A10,5I5) }
\end{array}
$$

The number of lines required to redefine material properties is not fixed. READN keeps reading lines until NI is zero: This is often accomplished by including a blank line. Therefore if READN is used, two blank lines appear at the end of this Data Set, one to indicate the end of information for READN and one to indicate the end of the Data Set. After reading all the lines prior to the blank line, the DUST code determines if the number of material types assigned equals the number requested in variable NCM. If there is a discrepancy, an error message is printed and the code is stopped. A check is also performed to insure that the assigned material types are between 1 and the number of materials.

An example of redefining nodes 90 - 99 as material type 3 follows.

$$
\begin{aligned}
& \text { NI NSEQ NADD NITYP NTYPAD } \\
& \begin{array}{llllll}
90 & 10 & 1 & 3 & 0
\end{array}
\end{aligned}
$$

\subsection{DATA SET 4: Output Specifications (Menu 4)}

This data set begins with 3 label lines, (end of previous Data Set, beginning of this Data set, and a label for the output print flags). The next set of lines define the output requested at each computation time. If more than 1000 computation times are requested, the user is required to only input print flags for the first 1000 time steps. This pattern of 1000 values is repeated until the maximum number of time steps. This flag has up to four values,

$0=$ print nothing,

$1=$ print concentrations;

$2=$ print 1 plus flux; and

$3=$ print 2 plus wasteform release data.

The input procedure is: 
BLANK LINE

DATA SET 4: Output Control Parameters

Print Control Parameters for each time step (NTI)

0000000001000000000100000000000000000000000000000100000000000000000000 0000000000000000000000000000010000000000000000000000000000000000000000 0000000001000000000000000000000000000000000000000000000000010000000000 0000000000000000000000000000000000000001000000000000000000000000000000 0000000000000000000100000000000000000000000000000000000000000000000001 0000000000000000000000000000000000000000000000000100000000000000000000 0000000000000000000000000000010000000000000000000000000000000000000000 0000000001000000000000000000000000000000000000000000000000010000000000 0000000000000000000000000000000000000001000000000000000000000000000000 0000000000000000000100000000000000000000000000000000000000000000000001 0000000000000000000000000000000000000000000000000100000000000000000000 0000000000000000000000000000010000000000000000000000000000000000000000 0000000001000000000000000000000000000000000000000000000000010000000000 0000000000000000000000000000000000000001000000000000000000000000000000 00000000000000000001

READ(5,3) LABEL

READ(5,3) LABEL

READ $(5,3)$ LABEL

30

READ(5,30) SL,(KPR(J),J=IMN,IMX)

FORMAT (A10,70I1)

The procedure for determining KPR is identical to that used in INPTI except the format is different. The variables IMN and IMX start as 1 and 70, respectively, and are continually redefined until IMX equals the number of computation time intervals (NTI).

The next set of variables specify the number of concentration and flux traces as well as the number of time steps between writing these variables to their respective output files. Concentration trace variables are written to TRACCND\&.DAT, while flux trace variables (flux, mass release and mass release rate) are written to TRACFXD\&.DAT. In the preceding statement, the \& identifies an isotope number. That is, information is written to trace files on an isotope specific basis. TRACCND1.DAT contains concentration traces at the specified locations for isotope 1. TRACCND2.DAT is for isotope 2, and so on. The final variable on that line, NSTPLCH defines the number of computational time steps between writing information to the file LEACHRL.DAT, which contains information on the release rate from each container for each release mechanism (rinse, diffusion, and dissolution, discussed in Section 9). In addition, if flux traces are specified, the code automatically creates a file for each isotope labeled BOUND\&.DAT. This file contains the mass flux at each flux trace location for possible use as the upstream boundary condition in a sequential run to represent the aquifer. This file can be quite large if there are a large number of wasteforms. A negative or 0 value for NSTPLCH prevents information from being written to this file. The format is:

Trace var $\quad 6 \quad 6 \quad 3$ 
If the number of concentration traces, NTRC, is non-zero, the nodal locations for the concentration traces are read by the code. These variables are read through the subroutine INPTI which reads integer values using the following format.

\begin{tabular}{rllllll|}
\hline Con $\operatorname{Tr}$ LOC & 10 & 20 & 30 & 40 & 50 & 60 \\
& & READ(5,10) SL,(NF(I),I=IMN,IMX) \\
& FORMAT(A10,14I5)
\end{tabular}

In the calling statement for INPTI, the dummy array NF is assigned to the array NTRCEC.

Similarly, if the number of flux traces, NTRF, is non-zero, the trace locations are read in by the code using the INPTI subroutine while using the array NF to store values for NTRCEF.

\subsection{DATA SET 5: Facility Co-ordinate Data (Menu 5)}

The first two lines in this Data Set are the label lines for the end of the previous set and the beginning of this set. The next line contains cross sectional area of the facility. This is needed for normalization purposes when representing the three dimensional system in one dimesnion. For example, if the facility is $10 \mathrm{~m}$ deep with a surface area of $100 \mathrm{~m}^{2}$, when defining the inventory to be used in the calculation, the facility inventory can be used. The concentration will be this inventory divided by the volume of the waste containing region (one dimensional distance which waste is emplaced multiplied by $100 \mathrm{~m}^{2}$ ).

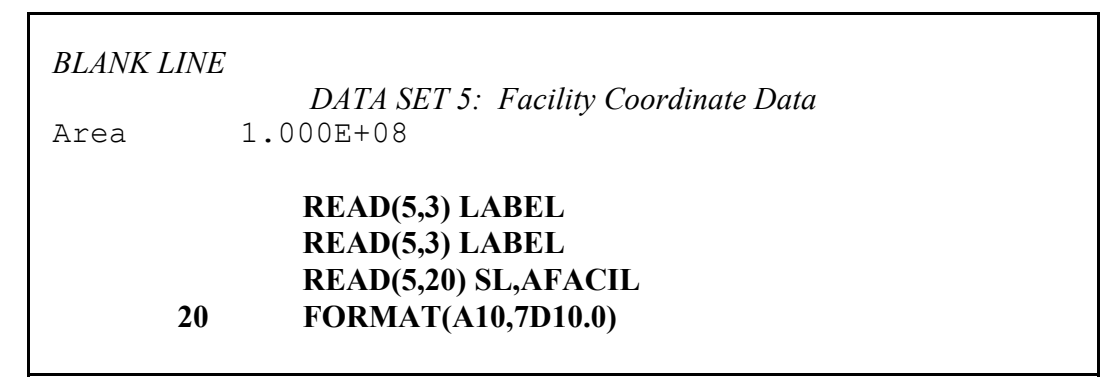

The procedure used to specify the size of each cell is accomplished using subroutine READR. The format statement in READR is: 


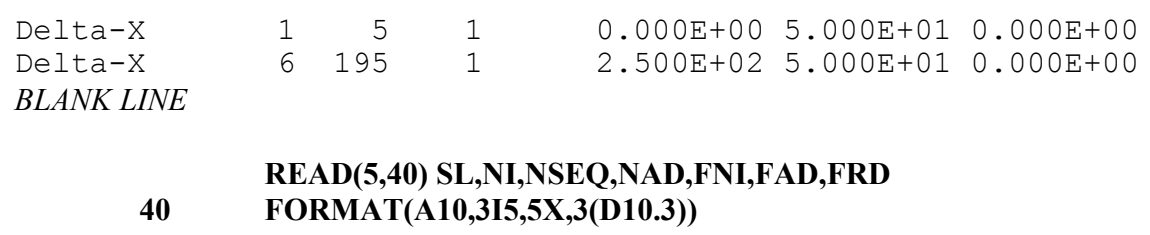

This places the first control volume between 0 and $25 \mathrm{~cm}$, the second between 25 and $75 \mathrm{~cm}$, etc. A complete discussion of the geometry definition for the finite difference model is in Appendix A.

\subsection{DATA SET 6: Initial and Boundary Conditions (Menu 6)}

The first two lines in this data set are label lines which indicate the completion of the previous data set and the beginning of this data set.

BLANK LINE

DATA SET 6: Initial and Boundary Conditions

READ(5,3) LABEL

READ(5,3) LABEL

The next set of lines are read by the subroutine READR and define the initial conditions for each iosotope at each computational point. The READ statements are:

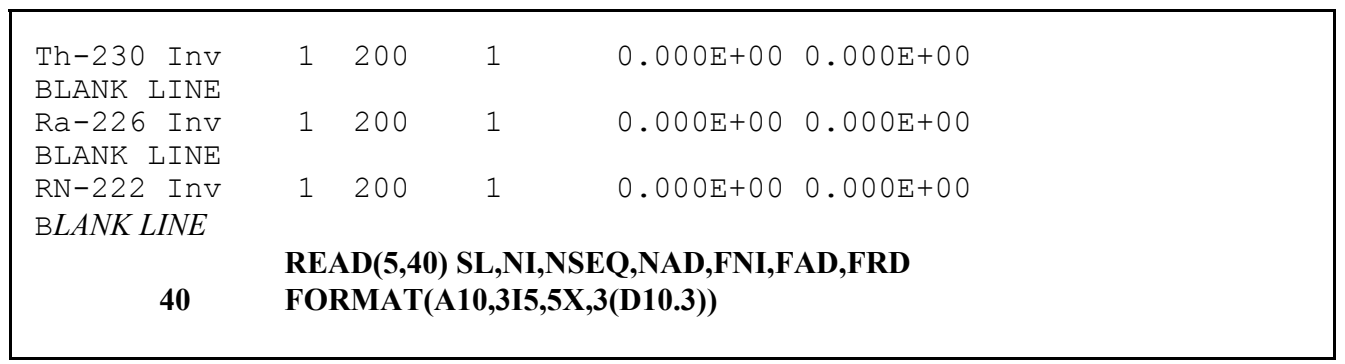

In this case, the values input into READR for FNI, FAD, and FRD are used to determine concentrations. 
Within READR, the READ statement is repeated until the value for NI is zero. Therefore, to end this segment of input a blank line is used. Again, the DUSTMS-D code checks to insure that all locations are given an initial value. Upon completion of defining the initial conditions for one isotope, the process is repeated for the next isotope.

The next set of lines define the boundary conditions. The boundary conditions can be defined via input from this file or from information in a separate auxiliary file. When defining the boundary conditions using this input file, the code user must specify the choice for the boundary condition at both boundaries for each isotope. Four different boundary conditions (concentration, total flux, advective flux, and dispersive flux, respectively) may be selected in the parameter IBFLAG. The boundary conditions are determined using a table with values that depend on time. The units used for the values depend on the boundary condition flag. However, if the mass flag (Menu 1) is set to indicate that mass values are input in Curies, the boundary conditions mass units must be Curies. The units of time required by the input tables is in years. The first input line defines the flags used to determine the boundary condition and the number of points used to specify the boundary condition in the tables for the first isotope. The boundary condition tables are input and the process is repeated for the remaining isotopes.

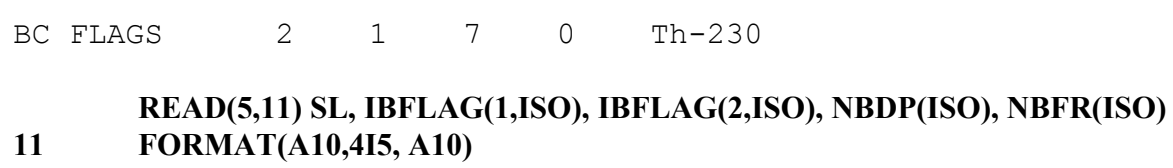

Note, the isotope name to the right of the integer input is not read by DUSTMS-D. The variable NBFR is a flag that determines the source of boundary condition input. If NBFR is one, input is read from auxiliary files called BOUND?.DAT where the ? is a dummy variable that ranges from $1,2, \ldots 9,0$ and represents the isotope number, with 0 representing isotope 10 . The BOUND?.DAT files are created automatically by DUST-MSD. The BOUND?.DAT files are used when using DUST-MS to simulate release from the disposal site, transport through the unsaturated zone and then transport in the saturated zone. This is accomplished in two steps. The first simulates waste forms, containers, and the disposal facility. Release is calculated and the DUST-MSD code writes an output file for each radionuclide in the simulation (BOUND?.DAT). The file contains the mass flux and the time at the bottom boundary. In this file, NBFR is set to 0 . In the second simulation, NBFR is 1 . The mass flux in the BOUND?.DAT files is used as the top boundary condition and transport through the aquifer is simulated. This approach conserves mass leaving the unsaturated zone and entering the saturated zone.

Even if NFBR is set to 1, input values are still needed for the top and bottom boundary conditions. However, the top boundary conditions in the input file are overwritten by the information in the BOUND?.DAT files. The next set of input values defines the boundary condition versus time using the subroutine INPTR2. INPTR2 is called to read the times for the top boundary (node 1) condition 
followed by the values at the top. Then, the times for the bottom boundary followed by the values at the bottom. INPTR2 is called by the following statement:

\section{CALL INPTR2(TBC(1,1,ISO),MXBDP,NBDP,I,MXBPR)}

In this example, TBC, time of the boundary condition is specified through input. Other variables in the call statement are MXBDP, the maximum boundary data points in a Table (currently 10 ), NBDP is the user specified number of points in the table, I is the boundary condition indicator (I $=1$, top boundary; $\mathrm{I}=2$, bottom boundary), and MXBPR is the maximum number of boundaries (currently 2). After reading the times in the table, the values are read using subroutine INPTR2.

The following example supplies the top and bottom boundary conditions for a single isotope. The procedure is repeated for each isotope.

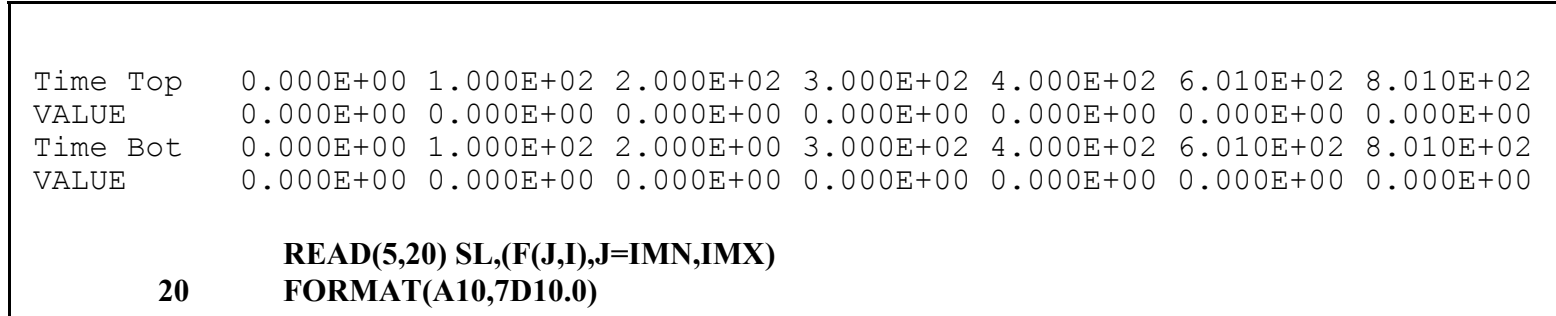

An example of specifying zero flux at the the top boundary and zero concentration at the bottom boundary for the duration of the problem is provided in Table 7.1.

\subsection{DATA SET 7: Water Flow Parameters (Menu 7)}

The first two lines in this data set are label lines which indicate the completion of the previous data set and the beginning of this data set. They are:

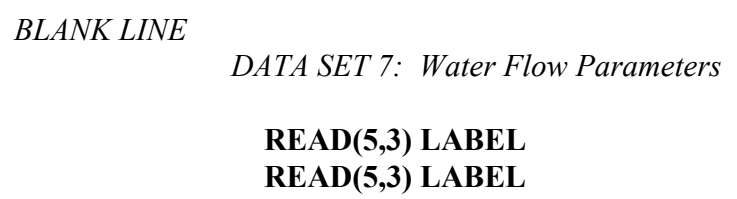

The Darcy velocity $(\mathrm{cm} / \mathrm{s})$ can be a function of time (yrs) and is determined by tabular input similar to that used to specify the boundary conditions. The moisture content may vary with location and therefore it is input similar to the approach used in specifying location. 
is:

The first data line specifies the number of points in the Darcy velocity versus time table and

\begin{tabular}{|lll|}
\hline Vel pts & 2 & \\
& & READ(5,10) SL,NVP \\
& $\mathbf{1 0}$ & FORMAT(A10,14I5) \\
\hline
\end{tabular}

The tables for Darcy velocity are read using the subroutine INPTR2 which was described in the beginning of this chapter and in the discussion of boundary conditions, Section 7.6. The times used in the table are read in first, seven to a line beginning in column 11. After all the times are input, the Darcy velocity values are read. The example provided in Table 7.1 requires two values for the velocity table and defines the Darcy velocity to be $2.316 \mathrm{E}-4 \mathrm{~cm} / \mathrm{s}$ for the first 1001 years Note, the time-dependent parameters (boundary conditions and velocity), should be defined at least as long as the maximum problem time (in this example, the maximum problem time is 300 years).

The moisture content is input using the subroutine READR. Use of this subroutine is also described in the beginning of this chapter. The format used in the read statement is:

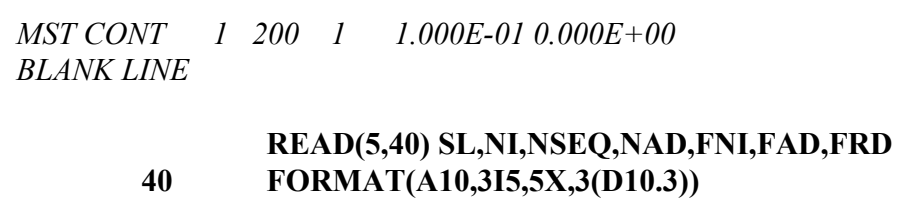

Here, the variables FNI, FAD, FRD refer to moisture content. In the example provided in Table 7.1, the moisture content is set to 0.1 at all 200 points. READR continues reading lines until NI is zero. This is accomplished in the example through a blank line as shown above. Therefore, there are two blank lines at the end of this data set.

\subsection{DATA SET 8: Container Parameters (Menu 8)}

As usual, the first two lines in this data set are label lines which indicate the completion of the previous data set and the beginning of this data set. They are:

BLANK LINE

DATA SET 8: Container Parameters

READ(5,3) LABEL

READ $(5,3)$ LABEL 
The next line specifies the number of containers, container types, and failure distribution to be modeled. The code is dimensioned to allow up to 300 containers and 20 container types. A container is used to define a location at which a wasteform resides. Each container may have a unique failure distribution. The number of container types indicates the number of different local failure rates. If local failure is not modeled, NCTYPE should be 1 (a value of 0 will be set to 1 by the code). The variable NDISTR defines the failure mode. If NDISTR is zero, all failures are instant for each container. If NDISTR is one, each container represents a system of containers and the fraction of this system of containers that have failed is determined from a uniform failure distribution. If NDISTR is two, the fraction of the system of containers that have failed is determined by a Gaussian distribution. When using distributed failures, it is strongly recommended that the flag for local failures is set to 1 . The local failure model was designed prior to allowing distributed failures and was used to examine the effects of a small fraction failing at earlier times than the prescribed failure time. Thus, the local failure model was designed to examine the same type of effects as the distributed failure models. Testing of distributed failures with local failures has not been performed and unexpected results may occur. Examples for each of these three types of failure distributions will be provide. The read statement is:

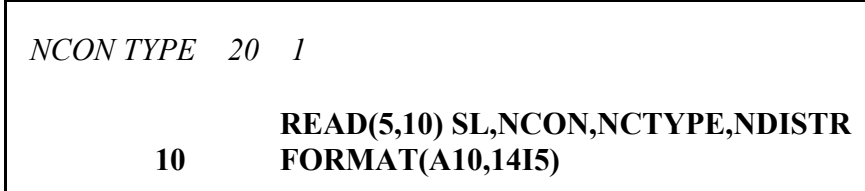

After these values are defined, the failure time (in years) for each container must be specified. This is accomplished in subroutine INPTR with the following statement:

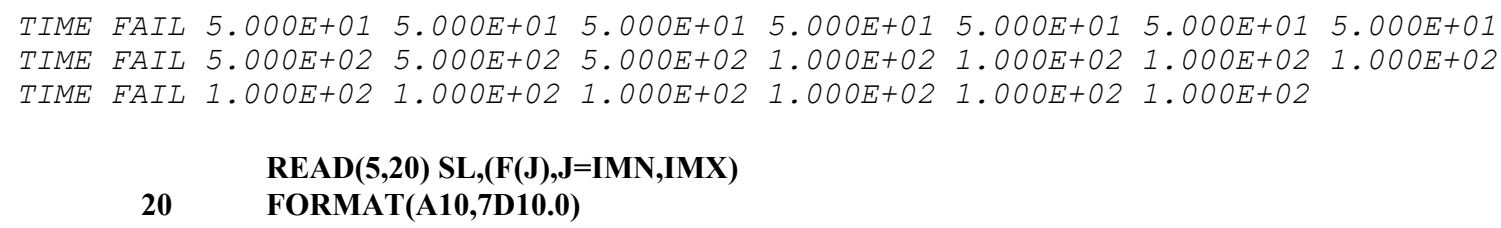

For the instant failure model (NDISTR $=0$ ) the next set of input values define the time at which the container was buried. Again, the input is obtained using the subroutine INPTR as follows. 


\begin{tabular}{rlllllll|} 
Time Bur & 1950. & 1952. & 1955. & 1961. & 1965. & 1968. & 1968. \\
Time Bur & 1975. & 1980. & 1981. & 1981. & 1985. & 1988. & 1988. \\
Time Bur & 1990. & 1990. & 1991. & 1991. & 1995. & 1998. & \\
& & & & & \\
& & & & & \\
\end{tabular}

The dummy array F represents the time to failure, TIMEF in this call to INTPR. In the examples of Table 7.1 there are 20 containers, therefore, there are three container failure time lines.

The DUSTMS-D model also permits localized (partial) failure of the containers. This requires further input. After specifying the time of failure, the user must specify if local failure is modeled for each separate container type (recall the minimum number of container types is 1 ). This is accomplished using the subroutine INPTI.

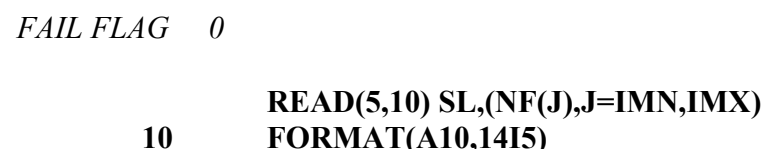

The dummy array NF is used to define the array IPIT.

With the array IPIT, the code cycles through each container type. If IPIT(J) is 1 , the flag indicates that local failure is modeled for the J-th container type. In this case, the parameters (defined in the discussion of Menu 8 in Chapter 6) that predict local corrosion must be input. Suggested values for these parameters are presented in Appendix D. The read statement is:

If IPIT( $\mathrm{J})$ is zero, the previous statement is not executed and input is not required. Therefore, to model only general corrosion, the user can set the number of container types, NCTYPE, to 1 and set IPIT(1) to zero. This was done in the example, Table 7.1. If the user desired to use localized corrosion on only a few containers, this could be accomplished by defining two container types. Container type 1 would have IPIT(1) equal zero and therefore would consider only general corrosion. Container type 2 would have IPIT(2) $=1$ and the local corrosion variables would be input only for this container. 
Container locations are specified using the subroutine INPTI. Each container must be given a unique location. In the example, there are 20 container locations specified by use of the following:

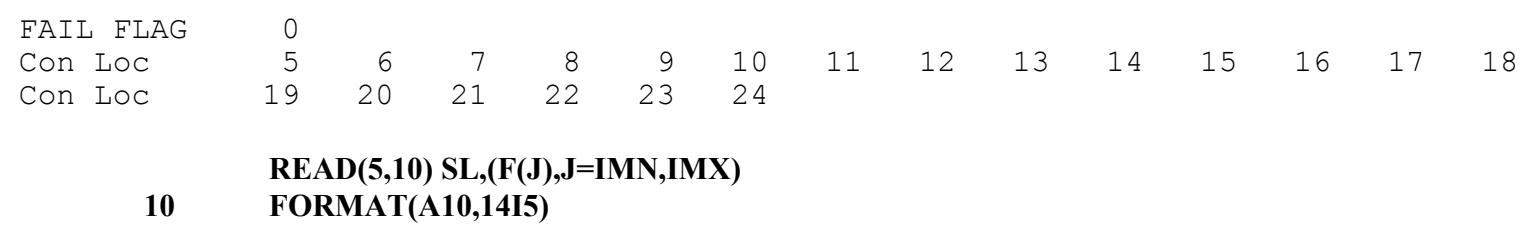

Here, the array $\mathrm{F}$ is used to define NELCON. For 53 containers, four input lines are required (three containing 14 values and one containing 11).

When more that one container types is defined, the user must specify the type of container in each container location. This is also accomplished using the subroutine INPTI. If only one container type is defined, the code automatically sets this parameter and input is not required. Specifying wasteform types requires an identical procedure. An example of this procedure is provided in Section 7.9.

$\underline{\text { Input for Distributed Container Failure Models. }}$

When using the distributed failure models, the input required for this data set changes to account for the additional data requirements. In DUSTMS-D, each container location is permitted a unique failure distribution. When the input variable NDISTR is 0 , the failure "distribution" is a single failure time. When NDISTR is 1 , the failure distribution for the container varies linearly over time starting and ending at user specified times after emplacement. If NDISTR is 2, the failure distribution is determined from a Gaussian distribution with a mean value and standard deviation specified by the user. The mean and standard deviation are specified from the time of emplacement. In addition, for distributed failure models (NDISTR $=1$ or 2), a fraction of the containers may be simulated to fail upon emplacement. Examples of data set 8 for each failure distribution are provided below.

Uniform Container Failure.

The following example considers two containers $(\mathrm{NCON}=2)$, one container type $($ ICTYPE $=1)$, and uniform failure (NDISTR $=1$ ). As specified in the line of input starting with NCON.

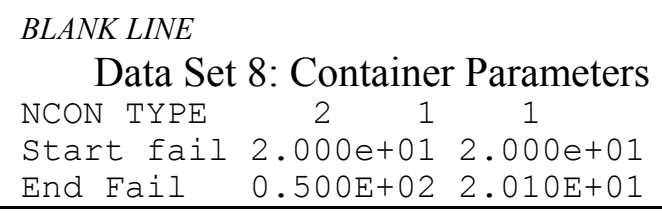




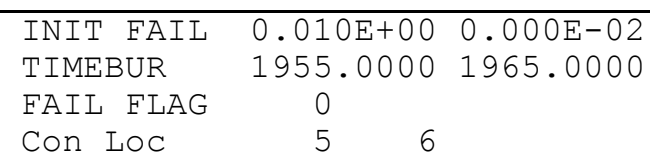

This input line has been discussed previously. The second line starting with Start Fail provides the time of failure after emplacement. Thus, both containers fail 20 years after emplacement. The third line gives the ending time for failures after emplacement. Container 1 completes it's failures at 50 years, while container 2 completes its failures after 20.1 years. Container 2 is very similar to an instant failure. In fact, if the time step is greater than 0.1 years, all of the containers fail over one time step. The fraction of containers that fail at emplacement must be less than 1 and they are defined in the line beginning with INIT Fail. In this case, 0.01 (i.e. 1\% ) of containers in the first location fail at emplacement. At location 2, all containers survive emplacement intact. The next line provides the time at which the burial occurs. In this example, the first container is buried in 1955 and therefore, starts the distributed failures in 1975 (20 years after emplacement). In the second location, the container is buried in 1965 and therefore starts the distributed failures in 1985. The Fail Flag and Con Loc lines define local failure and container locations as previously discussed. It is strongly recommended to use a failure flag of 0 when using distributed failures.

The variables on the lines Start Fail, End Fail, and INIT Fail are all read with subroutine INPTR using the following statements:

20

$$
\begin{aligned}
& \text { READ(5,20) SL,(F(J),J=IMN,IMX) } \\
& \text { FORMAT(A10,7D10.0) }
\end{aligned}
$$

If more than 7 containers are defined, the variables are input 7 to a line with the first 10 spaces being reserved for comments. Examples of this type of input were provided previously when discussing burial times.

\section{Gaussian Container Failure.}

The following example considers two containers $(\mathrm{NCON}=2)$, one container type (ICTYPE $=1)$, and uniform failure (NDISTR $=2$ ). As specified in the line of input starting with NCON.

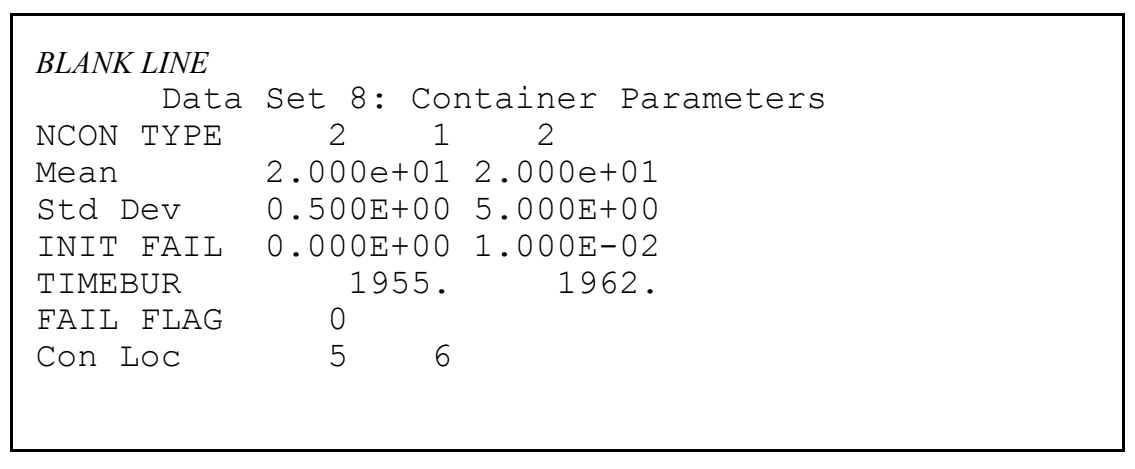


This input line has been discussed previously. The second line starting with Meanl provides the mean time of failure after emplacement. Thus, both containers have a mean failure time of 20 years after emplacement. The third line gives the standard deviation in failure times. Container 1 has a standard deviation in failure times of 0.5 years,, while container has a standard deviation of 5 years. To simulate instantaneous failure using this model, the standard deviation should be much less than the time step size. The fraction of containers that fail at emplacement must be less than 1 and they are defined in the line beginning with INIT Fail. In this case, a0.01 (i.e. 1\%) of containers in the second location fail at emplacement. At location 1, all containers survive emplacement intact. The next line provides the time at which the burial occurs. In this example, the first container is buried in 1955 and therefore, the mean failure time is 1975 (20 years after emplacement). In the second location, the container is buried in 1962 and therefore starts the distributed failures in 1982 . The Fail Flag and Con Loc lines define local failure and container locations as previously discussed. It is strongly recommended to use a failure flag of 0 when using distributed failures.

The variables on the lines Mean, Std Dev, and INIT Fail are all read with subroutine INPTR using the following statements:

20

$$
\begin{aligned}
& \text { READ(5,20) SL,(F(J),J=IMN,IMX) } \\
& \text { FORMAT(A10,7D10.0) }
\end{aligned}
$$

If more than 7 containers are defined, the variables are input 7 to a line with the first 10 spaces being reserved for comments. Examples of this type of input were provided previously when discussing burial times.

\subsection{DATA SET 9: Wasteform Leaching Parameters}

The first two lines in this data set are the label lines used to indicate the completion of the previous set and the beginning of this set. The third line contains the number of different waste types, currently 20 is the maximum number of waste types. The DUSTMS-D code assumes that there is one wasteform in each container. A waste type defines a unique set of release parameters. The first three input lines for this data set are:

$\begin{array}{|ll|}\text { BLANK LINE } & \\ \text { WF TYPE } 2 & \\ & \\ & \text { REATA SET 9: Wasteform Leaching Parameters } \\ & \text { READ(5,3) LABEL } \\ & \text { READ(5,10) SL, NWTYPE } \\ 10 & \text { FORMAT(A10,14I5) } \\ & \end{array}$


The next set of data defines the geometry of the waste form. The first line in this sequence is a label line that identifies each variable. In particular, for each waste form type the diffusion flag, and waste form geometry parameters must be defined.. DUSTMS-D allows six different waste for release models as follows:

\begin{tabular}{lll}
$\underline{\text { IDIFF }}$ & $\underline{\text { Model }}$ & Waste Form Geometry Parameter \\
\cline { 3 - 4 } 1 & Rectangular, 3-d analytical & Half-width, Half-height, Volume \\
2 & Cylindrical, 2-d analytical & Radius, Volume \\
3 & Spherical, 1-d analytical & Radius \\
4 & Rectangular, 1-d finite difference & Half-width, half-height, Volume \\
5 & Cylindrical, 1-d finite difference & Radius, Volume \\
& Spherical, 1-d finite difference & Radius
\end{tabular}

The units of length on these variables is $\mathrm{cm}$. If the above table states that a variable is required, a non-zero value must be used. The diffusion flag definition has changed from the DUST code and version 1.0 of DUST-MS. In addition to including more options, in earlier versions of the code, IDIFF $=0$ applied to the 2-d cylindrical analytical model and IDIFF $=1$ applied to the rectangular geometry waste form. Use of input decks that worked with previous versions of DUSTMS-D will either not work at all or may work and give different results due to the switch in the definition of the diffusion geometry flag. This problem can be overcome by setting the diffusion flag to the appropriate value for version 2 of DUSTMS-D as listed above..

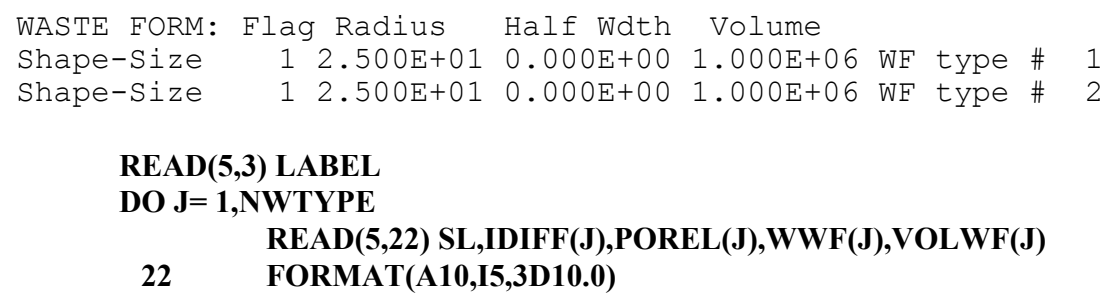

After specifying the geometry, the release parameters are input. The input routine reads a label line with identifiers for the parameters. Then the code reads one line for each isotope per wasteform type with the specified values.

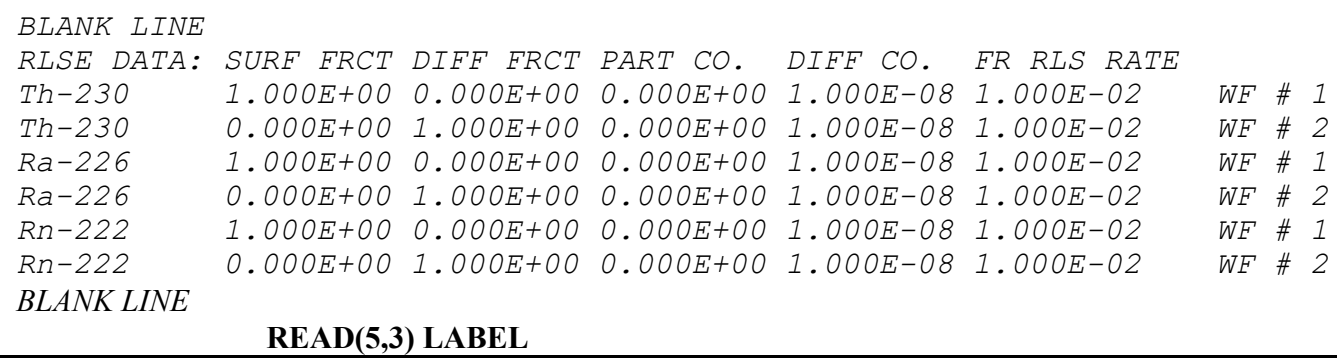

$\operatorname{READ}(5,3)$ LABEL 


\begin{tabular}{l} 
READ(5,3) LABEL \\
DO ISO = 1,NISO \\
DO J=1,NWTYPE \\
READ(5,20) SL,,SFRACT(J,ISO),PFRACT(J,ISO),PARTK(J,ISO), \\
DEFF(J,ISO), QFRACT(J,ISO) \\
$20 \quad$ FORMAT(A10,7D10.0) \\
\hline
\end{tabular}

The variables are:

SFRACT, fraction available for surface rinse;

PFRACT, fraction available for diffusion release;

PARTK, the wasteform partition coefficient;

DEFF, the wasteform diffusion coefficient; and

QFRACT, the annual fractional release rate.

These variables have been discussed in detail in Chapter 6. In Table 7.1, there are two waste types therefore, there are two sets of release parameters.

After defining the release parameters, the inventory for each isotope in each container must be specified. Each isotope in each container may have a unique inventory. This is accomplished using the subroutine INPTR which contains the following read statement:

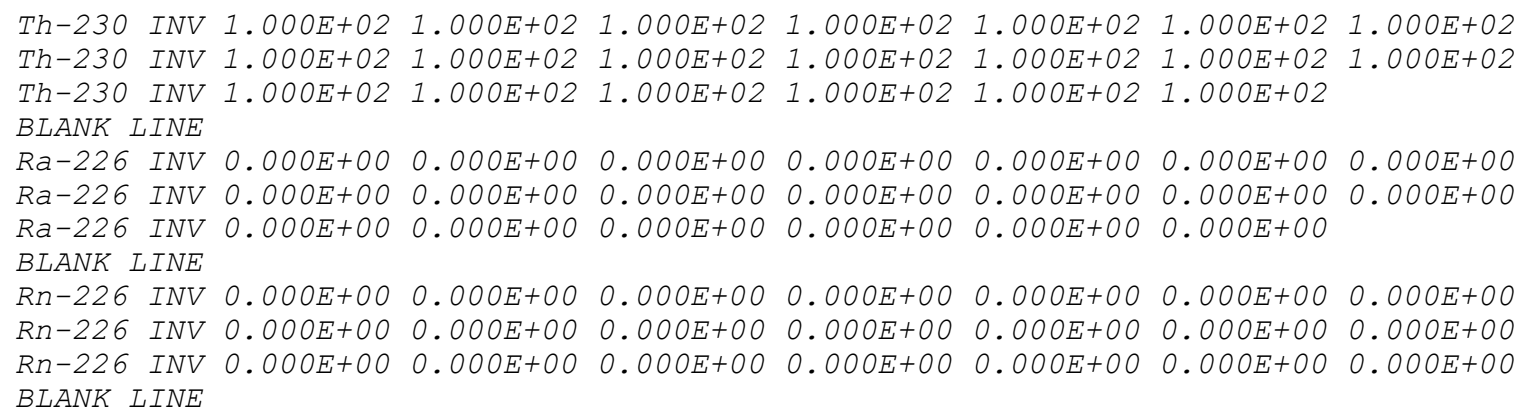

The dummy array F is used to determine the variable WTINIT used by DUSTMS-D. This read statement is repeated until all NCON values for initial mass are specified for the isotope.

The final input requirement is the assignment of wasteform release parameters to each container. This is only necessary if the number of waste types is greater than 1 . When required, the subroutine INPTI is used to read the input as follows: 


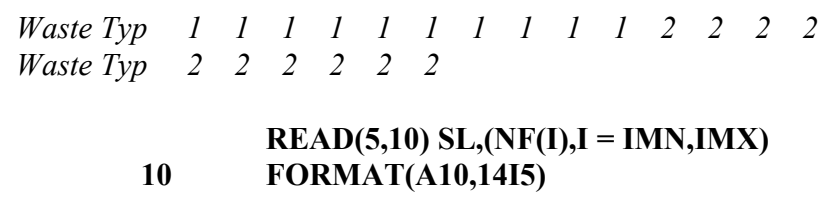

The dummy array NF is used to determine the variable IWTYPE used by DUST. The read statement is repeated until IMX $=$ NCON, the number of containers specified.

\subsection{DATA SET 10: External Sources}

DUSTMS-D permits an external source which is defined through tabular input of source strength versus time. The source is defined for each isotope. The first two lines in the data set are label lines identifying the end of the previous data set and the beginning of this set. The third line specifies the total number of sources, the number of different source profiles, and the number of data points required to create the table of source strength versus time. The read statements are:

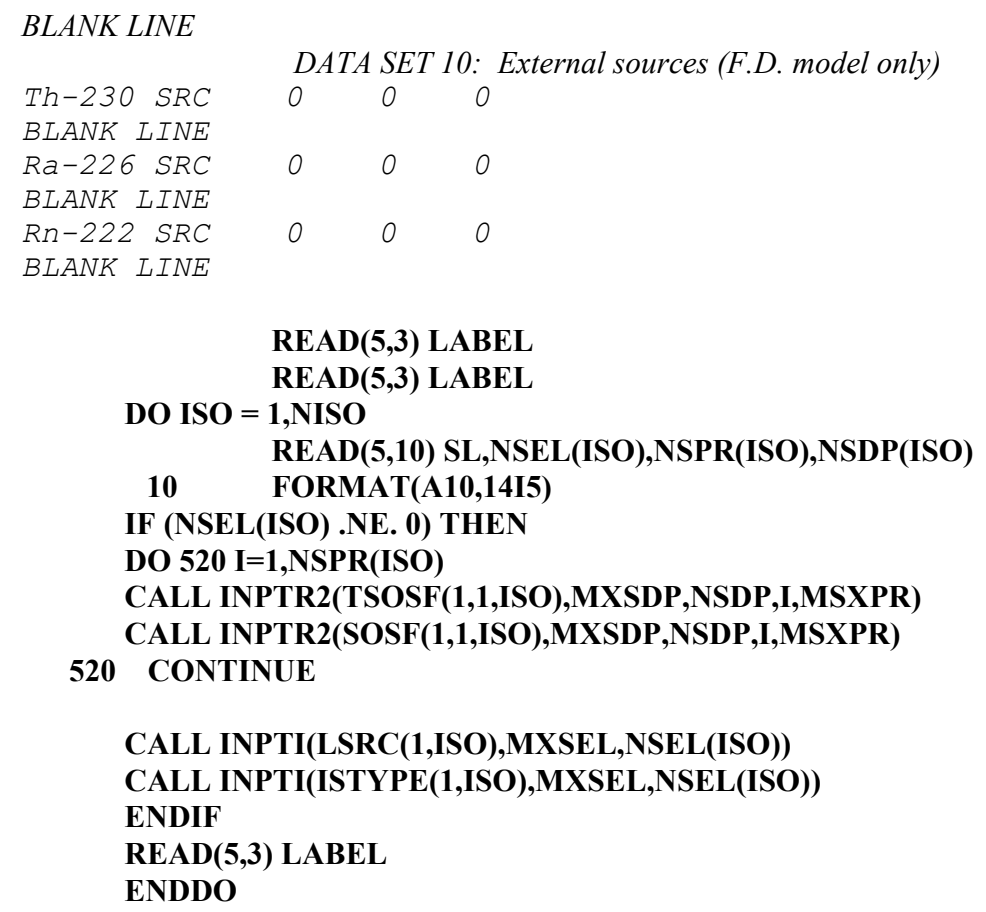


If the number of sources, NSEL(ISO), is zero, the required input is complete for that isotope and the input proceeds to the next isotope. This is the case in the example input deck, Table 7.1. If there are external sources, input to define the table of source strength versus time, the location of the sources, and which profile to use at each source location are required.

The table of source strength versus time is input for each different source profile, NSPR(ISO). The DUSTMS-D code uses the subroutine INPTR2 to translate the input into the variables required by DUSTMS-D. This input is analogous to defining velocity profiles or boundary conditions as a function of time. The read statement in INPTR2 is:

DUSTMS-D calls INPTR2 to obtain the times in the external source table for table number I. After obtaining the times for profile, I, the source strength is obtained using INPTR2. If the mass/activity flag requires mass units of curies, the source should be input in curies $/ \mathrm{cm}^{3} / \mathrm{yr}$. The code will translate this to grams $/ \mathrm{cm}^{3} / \mathrm{s}$ internally. After completing a profile, the index I is increased and the procedure repeated until all profiles are defined.

After completing the profiles, the source locations are specified. Source locations are defined using the same procedure when defining container locations. The subroutine INPTI is used to read in the locations as defined by control volume number.

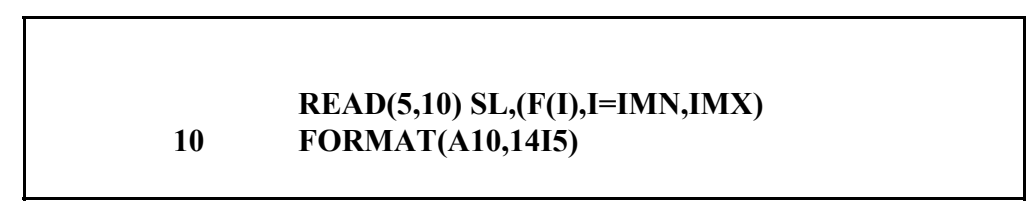

In this case, the dummy array $\mathrm{F}$ is used to assign values to the array LSRC used by DUSTMS-D.

The final piece of input required is the assignment of source type to each source location. The input is similar to specifying container and wasteform types. The routine INPTI is again used. In this case, the dummy variable F represents the variable ISTYPE. This input is required even if there is only one source type.

At this point, input is required for the next isotope and the process is repeated until the source definition for each isotope is complete.. 
An example of the input required for specifying an external source for only one of the isotopes being modeled is in Table 7.3. In this example, there are 3 source nodes, 2 source types, 4 data points in each table, the sources are located in nodes 20,30 and 40, and nodes 20 and 40 use profile 1 , while node 30 uses source type 2 . The type 1 source release rate is $1 \mathrm{E}-5$ for 0 to 10 years. After 10.01 years the release rate is zero. The type 2 source releases nothing for the first 10 years, increases to $1 \mathrm{E}-5$ after 10.01 years and remains constant until 1000 years. Linear interpolation is used to obtain source strength between data points in the table. If the value of time during the computation lies outside the range in the table, unpredictable results may occur.

Table 7.3 Input lines required to specify an external source for a single isotope.

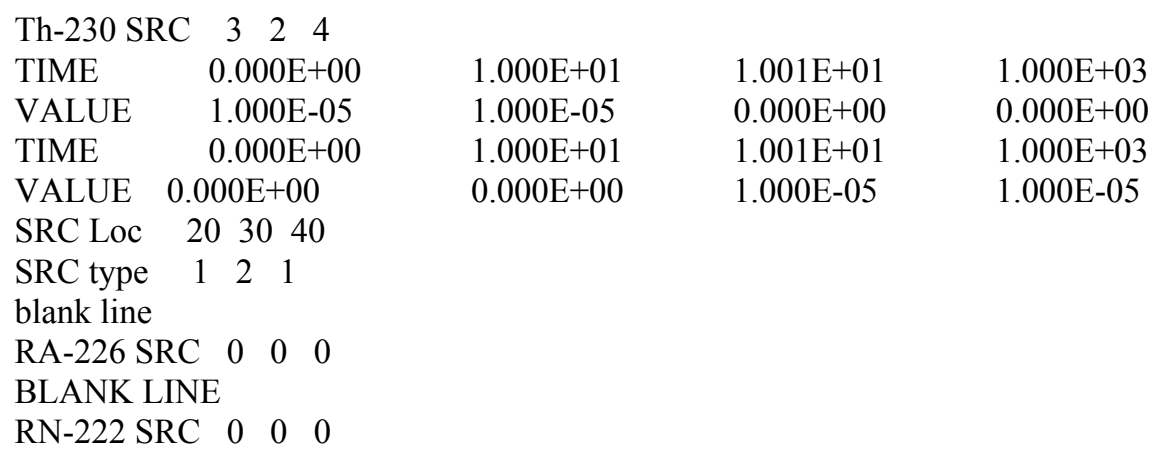




\section{DUST OUTPUT FILES}

DUST-MS creates a number of output files that are useful for tracking the predictions of the models. While running the DUST-MS code, the code prompts the user for the name of the primary output file. This file contains an echo check of the input as well as the output requested in the input file. In addition, up to five other types of files are written by the DUST-MS code. These files include: TRCCND?.DAT, TRCFXD?.DAT, CONCNT.DAT, LEACHRL.DAT and LEACHTMS.DAT. Where the question mark represents a number from $0-9$ representing the isotope. Isotope 10 would be TRCCND0.DAT. The content of each of file, along with a small example, is presented in this chapter.

\subsection{Primary Output File}

Table 8.1 contains an abbreviated output file for the FD test problem presented in Chapter 5 that was used for comparing the MCMC and FD models. In this problem there were 12 containers with failure times ranging from 0 to 40 years. Containers were located in every other node beginning at node 11 and ending at node 33 . Each node was $100 \mathrm{~cm}$. in length. Release from each wasteform was modeled assuming that the amount available for rinse and diffusion release was zero. The fractional release rate for all wasteforms was $5 \%$ per year. The initial condition was zero concentration everywhere. The boundary conditions were zero concentration at each boundary.

This main output file writes all information within 80 columns. This was done to provide a convenient method of viewing the files on standard desktop video display units.

The beginning of this file provides an echo check of the input file. Each of the ten data sets are printed for review. The data sets are: (1) Problem title and definition; (2) Time parameters; (3) Material properties; (4) Output control parameters, including trace locations; (5) Nodal coordinates and facility dimensions; (6) Initial and Boundary Conditions; (7) Water flow parameters (Darcy velocity and moisture content); (8) Container parameters; (9) Wasteform parameters; and (10) Source/Sink parameters. In the output, all automatic generation of initial conditions, node locations, and moisture content are expanded to print the specified value at each location as seen in Table 8.1. The structure of this file is identical for the MCMC model, however, the details are slightly different due to the different input requirements of the models.

After completion of the input check, the code prints a message that input is complete and that the calculation is about to begin. At this point, output is printed every time step at which the input value for KPR is non-zero. In this example, KPR for the first time step was 3. Therefore, a full output of concentration, flux, facility mass release rate, container performance and wasteform release is printed. 
Table 8.1 Typical primary output file when the FD transport model is used

TITLE: FD MODEL $-\mathrm{H}-3, \mathrm{MC}=0.2, \mathrm{VDAR}=1.58 \mathrm{E}-6, \mathrm{MLT}$ CONT

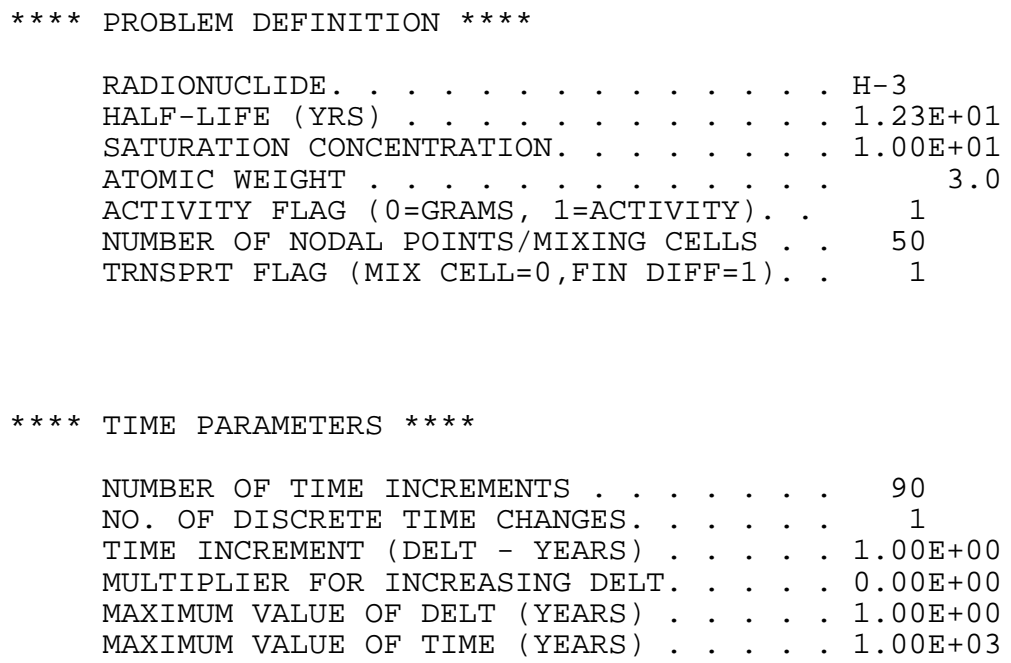


Table 8.1 Typical primary output file when the FD transport model is used

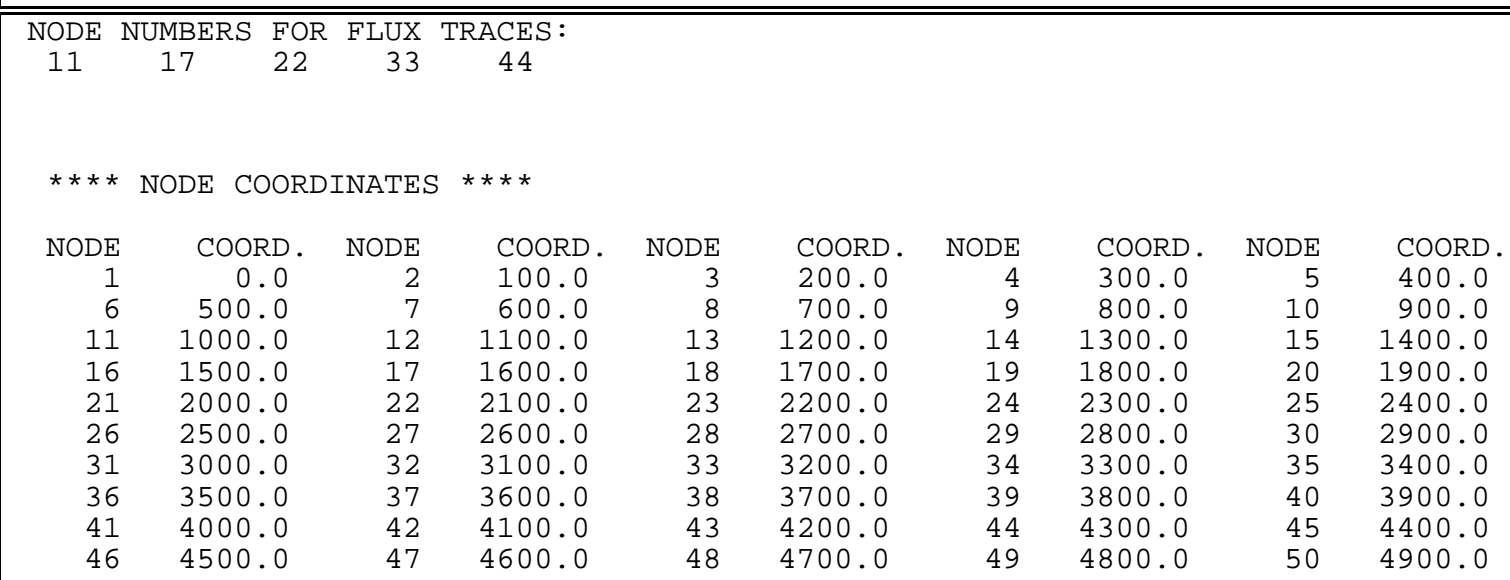

FACILITY DIMENSIONS

$\begin{array}{rrc}\text { FACILITY HEIGHT } & \text { X-SECT. AREA } & \text { VOL.OF FACILITY } \\ 4.900 \mathrm{E}+03 & 9.850 \mathrm{E}+03 & 4.826 \mathrm{E}+07\end{array}$

INITIAL CONCENTRATION AT EACH NODE:

NODE CONCENTR. NODE CONCENTR. NODE CONCENTR. NODE CONCENTR. NODE CONCENTR.

$\begin{array}{rrrrrrrrrr}1 & 0.0 \mathrm{E}+00 & 2 & 0.0 \mathrm{E}+00 & 3 & 0.0 \mathrm{E}+00 & 4 & 0.0 \mathrm{E}+00 & 5 & 0.0 \mathrm{E}+00 \\ 6 & 0.0 \mathrm{E}+00 & 7 & 0.0 \mathrm{E}+00 & 8 & 0.0 \mathrm{E}+00 & 9 & 0.0 \mathrm{E}+00 & 10 & 0.0 \mathrm{E}+00 \\ 11 & 0.0 \mathrm{E}+00 & 12 & 0.0 \mathrm{E}+00 & 13 & 0.0 \mathrm{E}+00 & 14 & 0.0 \mathrm{E}+00 & 15 & 0.0 \mathrm{E}+00 \\ 16 & 0.0 \mathrm{E}+00 & 17 & 0.0 \mathrm{E}+00 & 18 & 0.0 \mathrm{E}+00 & 19 & 0.0 \mathrm{E}+00 & 20 & 0.0 \mathrm{E}+00 \\ 21 & 0.0 \mathrm{E}+00 & 22 & 0.0 \mathrm{E}+00 & 23 & 0.0 \mathrm{E}+00 & 24 & 0.0 \mathrm{E}+00 & 25 & 0.0 \mathrm{E}+00 \\ 26 & 0.0 \mathrm{E}+00 & 27 & 0.0 \mathrm{E}+00 & 28 & 0.0 \mathrm{E}+00 & 29 & 0.0 \mathrm{E}+00 & 30 & 0.0 \mathrm{E}+00 \\ 31 & 0.0 \mathrm{E}+00 & 32 & 0.0 \mathrm{E}+00 & 33 & 0.0 \mathrm{E}+00 & 34 & 0.0 \mathrm{E}+00 & 35 & 0.0 \mathrm{E}+00 \\ 36 & 0.0 \mathrm{E}+00 & 37 & 0.0 \mathrm{E}+00 & 38 & 0.0 \mathrm{E}+00 & 39 & 0.0 \mathrm{E}+00 & 40 & 0.0 \mathrm{E}+00 \\ 41 & 0.0 \mathrm{E}+00 & 42 & 0.0 \mathrm{E}+00 & 43 & 0.0 \mathrm{E}+00 & 44 & 0.0 \mathrm{E}+00 & 45 & 0.0 \mathrm{E}+00 \\ 46 & 0.0 \mathrm{E}+00 & 47 & 0.0 \mathrm{E}+00 & 48 & 0.0 \mathrm{E}+00 & 49 & 0.0 \mathrm{E}+00 & 50 & 0.0 \mathrm{E}+00\end{array}$

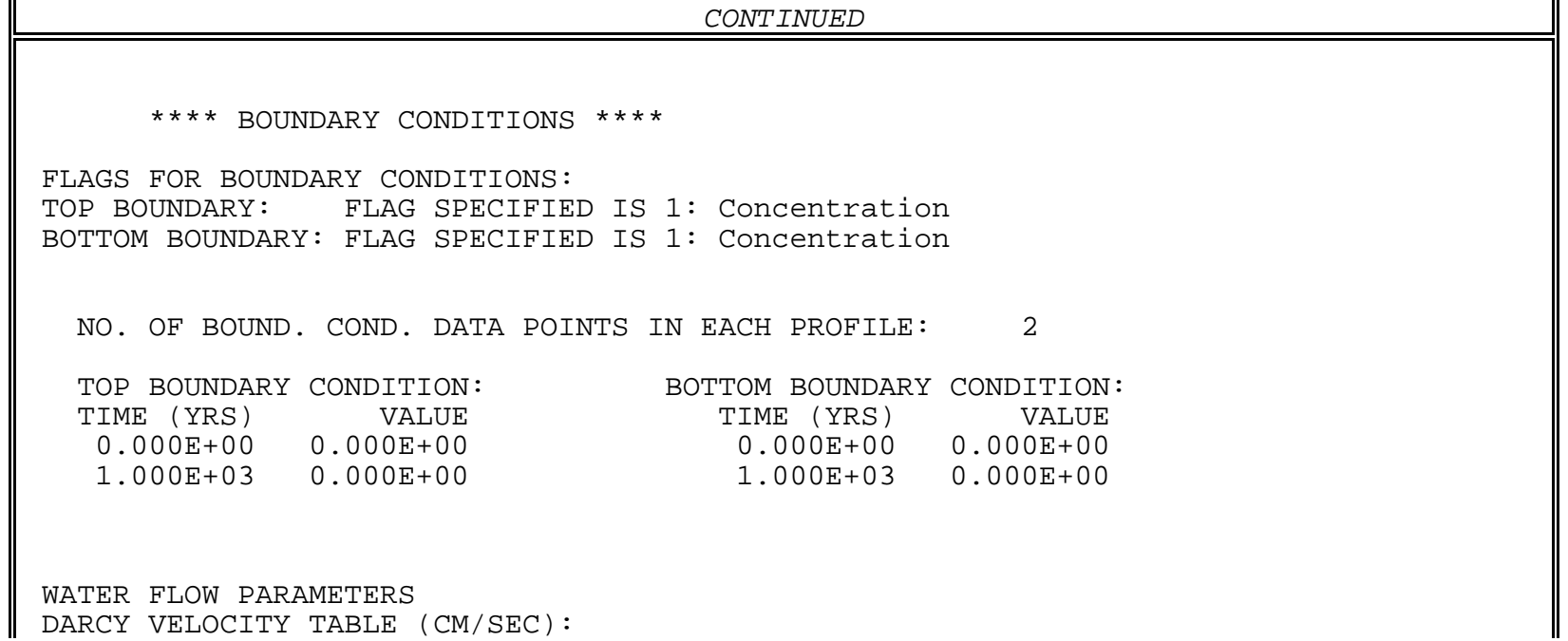


Table 8.1 Typical primary output file when the FD transport model is used

\begin{tabular}{|c|c|c|c|c|c|c|c|c|c|}
\hline \multicolumn{2}{|c|}{$\begin{array}{r}\text { TIME (YRS) } \\
0.000 \mathrm{E}+00 \\
1.001 \mathrm{E}+03\end{array}$} & & $\begin{array}{l}\text { VDAR } \\
7 \mathrm{E}-06 \\
7 \mathrm{E}-06\end{array}$ & & & & & & \\
\hline \multicolumn{10}{|c|}{ MOISTURE CONTENT } \\
\hline NODE & THETA & NODE & THETA & NODE & THETA & NODE & THETA & NODE & THETA \\
\hline 1 & 0.20 & 2 & 0.20 & 3 & 0.20 & 4 & 0.20 & 5 & 0.20 \\
\hline 6 & 0.20 & 7 & 0.20 & 8 & 0.20 & 9 & 0.20 & 10 & 0.20 \\
\hline 11 & 0.20 & 12 & 0.20 & 13 & 0.20 & 14 & 0.20 & 15 & 0.20 \\
\hline 16 & 0.20 & 17 & 0.20 & 18 & 0.20 & 19 & 0.20 & 20 & 0.20 \\
\hline 21 & 0.20 & 22 & 0.20 & 23 & 0.20 & 24 & 0.20 & 25 & 0.20 \\
\hline 26 & 0.20 & 27 & 0.20 & 28 & 0.20 & 29 & 0.20 & 30 & 0.20 \\
\hline 31 & 0.20 & 32 & 0.20 & 33 & 0.20 & 34 & 0.20 & 35 & 0.20 \\
\hline 36 & 0.20 & 37 & 0.20 & 38 & 0.20 & 39 & 0.20 & 40 & 0.20 \\
\hline 41 & 0.20 & 42 & 0.20 & 43 & 0.20 & 44 & 0.20 & 45 & 0.20 \\
\hline 46 & 0.20 & 47 & 0.20 & 48 & 0.20 & 49 & 0.20 & 50 & 0.20 \\
\hline
\end{tabular}

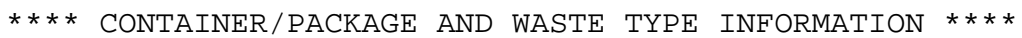

NO. OF WASTE CONTAINERS/PACKAGES . . . . . . . . 12

NO. OF CONTAINER/PACKAGE TYPES • . • . • . • . . 1

TIME OF BREACH (YRS) FOR EACH CONTAINER

$\begin{array}{rrrrrrrr}\text { CONT. } & \text { TIME } & \text { CONT. } & \text { TIME } & \text { CONT. } & \text { TIME } & \text { CONT. } & \text { TIME } \\ 1 & 0.00 \mathrm{E}+00 & 2 & 1.00 \mathrm{E}+01 & 3 & 2.00 \mathrm{E}+01 & 4 & 3.00 \mathrm{E}+01 \\ 5 & 4.00 \mathrm{E}+01 & 6 & 0.00 \mathrm{E}+00 & 7 & 1.00 \mathrm{E}+01 & 8 & 1.00 \mathrm{E}+01 \\ 9 & 2.00 \mathrm{E}+01 & 10 & 2.00 \mathrm{E}+01 & 11 & 0.00 \mathrm{E}+00 & 12 & 3.00 \mathrm{E}+01\end{array}$

CONT INUED

PARAMETERS FOR MODELING LOCALIZED FAILURE

CONT.TYPE THICKNESS PARAM N PARAM $\mathrm{K}$ AREA ASCALE PITS

1 - - - - LOCALIZED FAILURE NOT MODELED FOR THIS CONTAINER

LOCATION AND CONTAINER/PACKAGE TYPE

$\begin{array}{rrrrrrrrrrrc}\text { CONT } & \text { NODE } & \text { TYPE } & \text { CONT } & \text { NODE } & \text { TYPE } & \text { CONT } & \text { NODE } & \text { TYPE } & \text { CONT } & \text { NODE } & \text { TYPE } \\ 1 & 11 & 1 & 2 & 13 & 1 & 3 & 15 & 1 & 4 & 17 & 1 \\ 5 & 19 & 1 & 6 & 21 & 1 & 7 & 23 & 1 & 8 & 25 & 1 \\ 9 & 27 & 1 & 10 & 29 & 1 & 11 & 31 & 1 & 12 & 33 & 1\end{array}$

$\star \star \star *$ WASTE FORM PARAMETERS $\star * \star \star$

TYPE DIFF FLAG SURF FRCT PORE FRCT BULK FRCT PART COEF DIFF COEF FR REL RATE

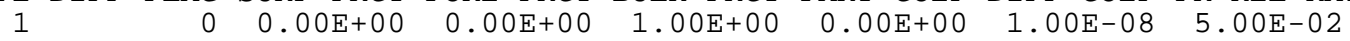

$\star \star \star *$ WASTEFORM DIMENSIONS $\star \star \star \star *$

TYPE PORE LEN WIDTH WF VOL WF 
Table 8.1 Typical primary output file when the FD transport model is used
1
$2.50 \mathrm{E}+01$
$0.00 \mathrm{E}+00$
$2.50 \mathrm{E}+07$

IN THIS SIMULATION MASS UNITS ARE IN CURIES

NODE NO. AND WASTE TYPE SOURCE AMOUNTS :

$\begin{array}{rrrrrrr}\text { I } & \text { NODE NO. WASTE } & \text { TYPE } & \text { SURF MASS } & \text { PORE MASS } & \text { BULK MASS } & \text { TOTAL MASS } \\ 1 & 11 & 1 & 0.00 \mathrm{E}+00 & 0.00 \mathrm{E}+00 & 8.33 \mathrm{E}-02 & 8.33 \mathrm{E}-02 \\ 2 & 13 & 1 & 0.00 \mathrm{E}+00 & 0.00 \mathrm{E}+00 & 8.33 \mathrm{E}-02 & 8.33 \mathrm{E}-02 \\ 3 & 15 & 1 & 0.00 \mathrm{E}+00 & 0.00 \mathrm{E}+00 & 8.33 \mathrm{E}-02 & 8.33 \mathrm{E}-02 \\ 4 & 17 & 1 & 0.00 \mathrm{E}+00 & 0.00 \mathrm{E}+00 & 8.33 \mathrm{E}-02 & 8.33 \mathrm{E}-02 \\ 5 & 19 & 1 & 0.00 \mathrm{E}+00 & 0.00 \mathrm{E}+00 & 8.33 \mathrm{E}-02 & 8.33 \mathrm{E}-02 \\ 6 & 21 & 1 & 0.00 \mathrm{E}+00 & 0.00 \mathrm{E}+00 & 8.33 \mathrm{E}-02 & 8.33 \mathrm{E}-02 \\ 7 & 23 & 1 & 0.00 \mathrm{E}+00 & 0.00 \mathrm{E}+00 & 8.33 \mathrm{E}-02 & 8.33 \mathrm{E}-02 \\ 8 & 25 & 1 & 0.00 \mathrm{E}+00 & 0.00 \mathrm{E}+00 & 8.33 \mathrm{E}-02 & 8.33 \mathrm{E}-02 \\ 9 & 27 & 1 & 0.00 \mathrm{E}+00 & 0.00 \mathrm{E}+00 & 8.33 \mathrm{E}-02 & 8.33 \mathrm{E}-02 \\ 10 & 29 & 1 & 0.00 \mathrm{E}+00 & 0.00 \mathrm{E}+00 & 8.33 \mathrm{E}-02 & 8.33 \mathrm{E}-02 \\ 11 & 31 & 1 & 0.00 \mathrm{E}+00 & 0.00 \mathrm{E}+00 & 8.33 \mathrm{E}-02 & 8.33 \mathrm{E}-02 \\ 12 & 33 & 1 & 0.00 \mathrm{E}+00 & 0.00 \mathrm{E}+00 & 8.33 \mathrm{E}-02 & 8.33 \mathrm{E}-02\end{array}$

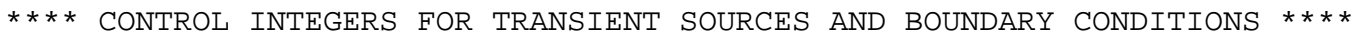

NO. OF SOURCE/SINK ELEMENTS. . . . . . . . . . . 0

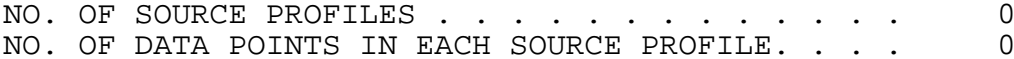

CONTINUED

COMPLETION OF INPUT ROUTINE

BEGINNING OF CALCULATION

OUTPUt TABLE 1.. CONCENTRATIONS AT TIME $=1.0000 \mathrm{E}+00,($ DELT $=1.0000 \mathrm{E}+00)$

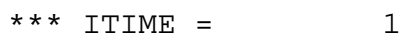

NODE I CONCENTRATION (Ci/ $\left.\mathrm{cm}^{\wedge} 3\right)$ AT NODES I, I+1, . , I+5

$\begin{array}{rlllll}1 & 0.0000 \mathrm{E}+00 & 0.0000 \mathrm{E}+00 & 0.0000 \mathrm{E}+00 & 0.0000 \mathrm{E}+00 & 0.0000 \mathrm{E}+00 \\ 6 & 0.0000 \mathrm{E}+00 & 0.0000 \mathrm{E}+00 & 0.0000 \mathrm{E}+00 & 0.0000 \mathrm{E}+00 & 0.0000 \mathrm{E}+00 \\ 11 & 1.7118 \mathrm{E}-09 & 3.5691 \mathrm{E}-10 & 7.4417 \mathrm{E}-11 & 1.5516 \mathrm{E}-11 & 3.2352 \mathrm{E}-12 \\ 16 & 6.7454 \mathrm{E}-13 & 1.4064 \mathrm{E}-13 & 2.9324 \mathrm{E}-14 & 6.1142 \mathrm{E}-15 & 1.2748 \mathrm{E}-15 \\ 21 & 1.7118 \mathrm{E}-09 & 3.5691 \mathrm{E}-10 & 7.4417 \mathrm{E}-11 & 1.5516 \mathrm{E}-11 & 3.2352 \mathrm{E}-12 \\ 26 & 6.7454 \mathrm{E}-13 & 1.4064 \mathrm{E}-13 & 2.9324 \mathrm{E}-14 & 6.1142 \mathrm{E}-15 & 1.2748 \mathrm{E}-15 \\ 31 & 1.7118 \mathrm{E}-09 & 3.5691 \mathrm{E}-10 & 7.4417 \mathrm{E}-11 & 1.5516 \mathrm{E}-11 & 3.2352 \mathrm{E}-12 \\ 36 & 6.7454 \mathrm{E}-13 & 1.4064 \mathrm{E}-13 & 2.9324 \mathrm{E}-14 & 6.1142 \mathrm{E}-15 & 1.2748 \mathrm{E}-15 \\ 41 & 2.6580 \mathrm{E}-16 & 5.5421 \mathrm{E}-17 & 1.1555 \mathrm{E}-17 & 2.4093 \mathrm{E}-18 & 5.0235 \mathrm{E}-19\end{array}$




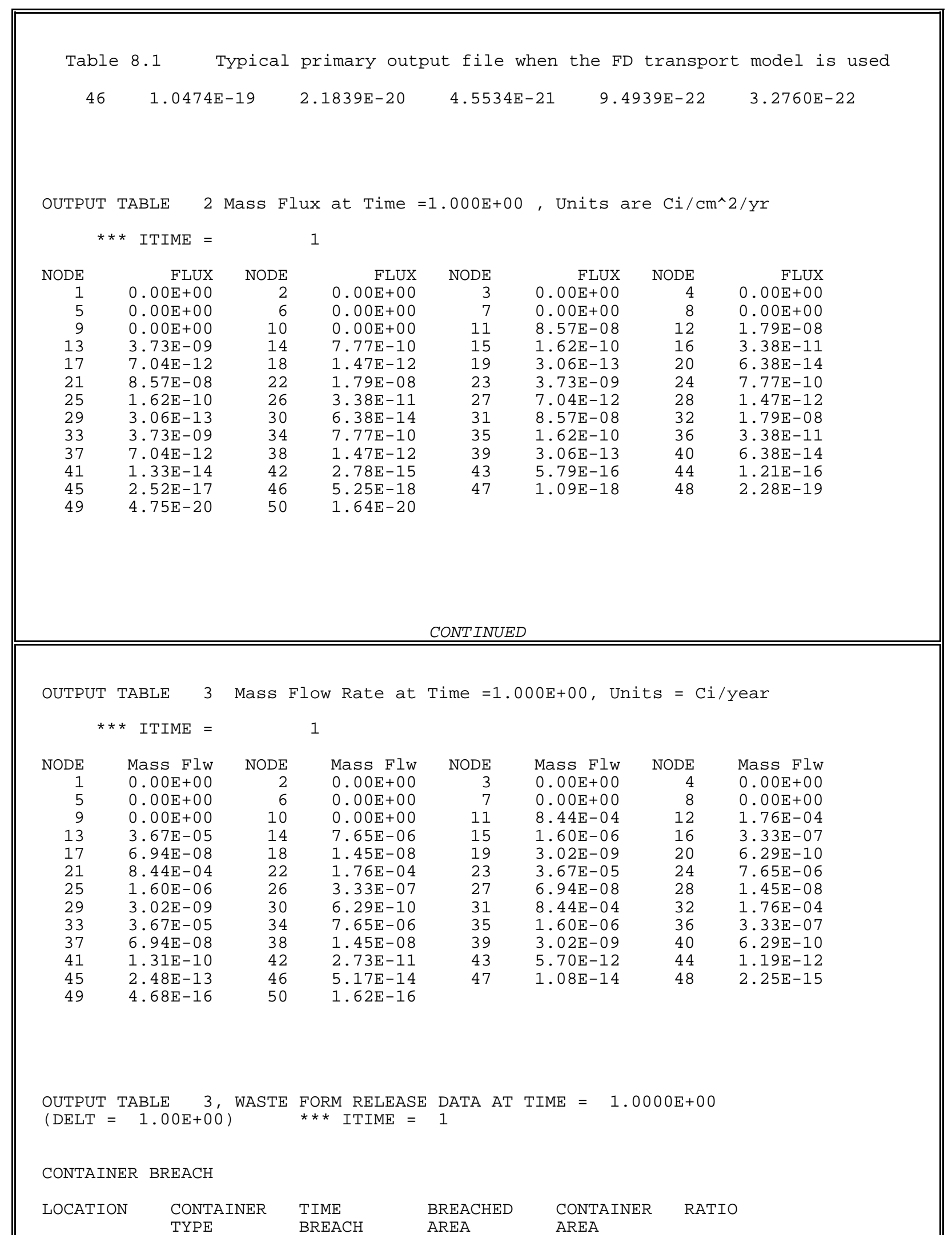




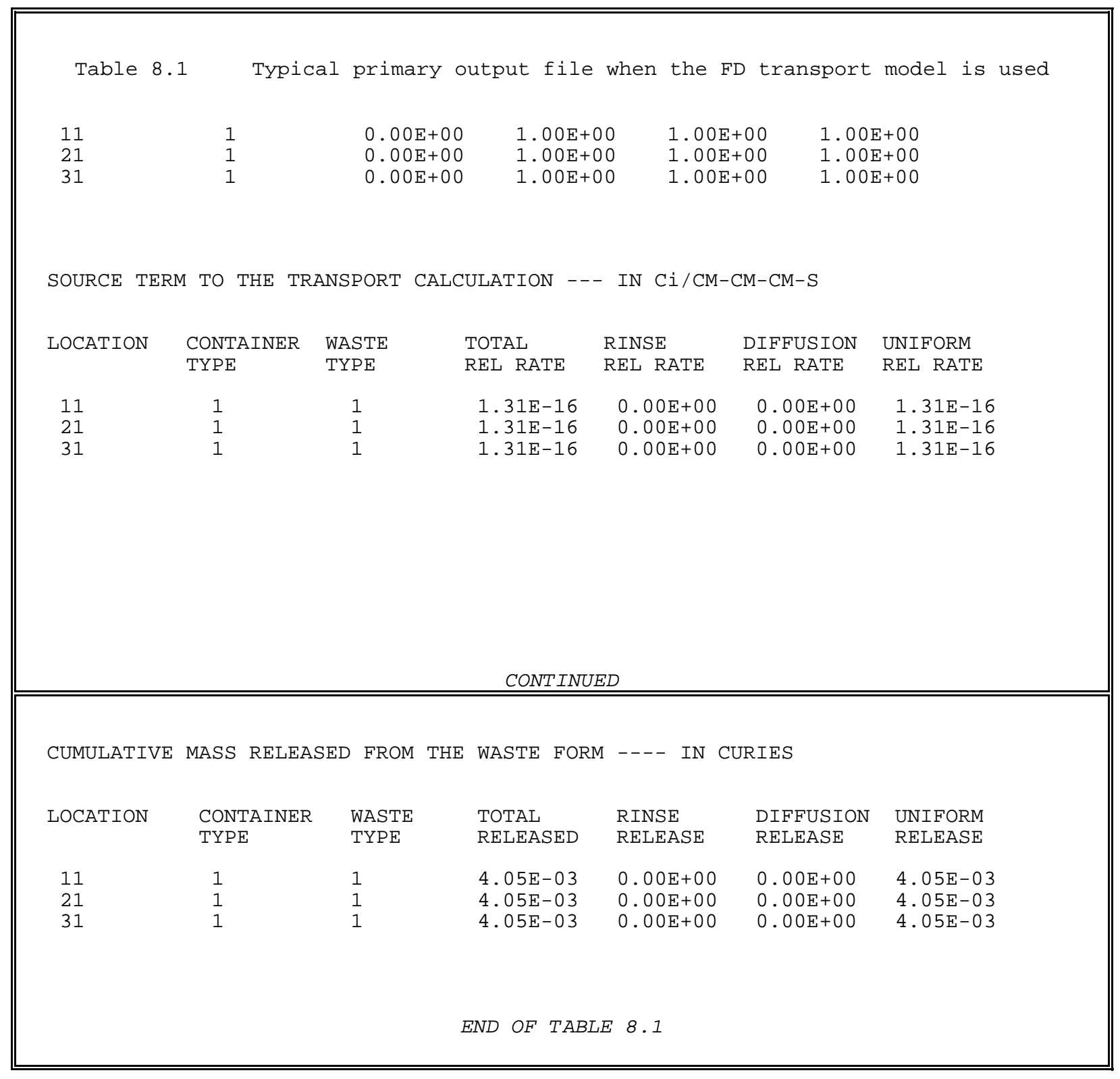


In Table 8.1 the first output from the calculation is the concentration at every point. These concentrations are also written to the file CONCNT.DAT which may be used by the graphics program GRAFXT. In the sample problem, containers in nodes 11, 21, and 31 all failed at the beginning of the problem, $($ Time $=0)$. From the concentrations we see that they are largest in these three nodes, as expected. Output for this time step would stop here if KPR $=1$.

Following the concentrations in Table 8.1 is the flux at each location. Again, highest fluxes occur at the source locations. Notice that the flux from node 11 is positive, i.e., flow is downstream only and there is no upstream dispersion. This is caused by the use of the upstream differencing and the use of zero values for dispersion and diffusion terms. This is the reason the concentration in nodes 1 - 10 are zero. Output for this time step would stop here if KPR $=2$.

Following the fluxes, container breach information and wasteform release information is presented only for the containers that have failed. In this example, total failure occurs at time $=0$ for containers in nodes 11,21 , and 31 . For these containers, localized corrosion is not modeled, therefore, the container area and breached area are given default values of 1.0. If localized failure is modeled, the container and breached area would be printed. After the container information, the wasteform release rates and total mass released for all three containers are printed.

Further output is controlled by the value of KPR at each time step.

The output file for the MCMC model is slightly different once the calculation begins. In the MCMC model, the concentration, flux and mass flow rate are calculated only at the nodes specified through the use of the Trace Nodes. Further, since this model does not permit localized failure, all container failure times are known from the input. Also, wasteform release rates are known from input, either rinse release is specified, all released immediately after breach, or the fractional release rate is known. For these reasons, information on container failure time and wasteform release rate are not printed in the output.

If the output flag KPR is non-zero at a time step, and the MCMC model is specified, the location, concentration, flux, and mass flow rate at each requested computational point is printed. An example, is provided in Table 8.2, where nodes 1, 7, 12,23, and 34 have been designated as trace nodes.

\subsection{Concentration Trace File (TRCCND?.DAT)}

The concentration trace file is intended primarily for graphical output. It can be used directly by the programs GRAFMS for visual output on the video screen as well as imported into graphics programs or spread sheets for hard copy output. It contains the concentrations at the requested trace locations as a function of time. 
Table 8.2 Typical primary output during the computation when the MCMC transport model is used

BEGINNING OF CALCULATION

OUTPUT TABLE 1, Concentration and Flux data at Time $=1.0000 \mathrm{E}+00$

Mass Units are $\mathrm{Ci} \quad * \star \star \operatorname{ITIME}=1$

MIXING BATH MODEL PREDICTED CONCENTRATIONS AND FLUXES

$\begin{array}{rlccc}\text { LOC } & \text { DIST }(\mathrm{cm}) & \text { CONC Ci/cm^3 } & \text { FLUX Ci/cm^2/yr } & \text { FLOW RATE Ci/yr } \\ 1 & 1.00 \mathrm{E}+02 & 1.94 \mathrm{E}-09 & 9.71 \mathrm{E}-08 & 9.57 \mathrm{E}-04 \\ 7 & 7.00 \mathrm{E}+02 & 1.60 \mathrm{E}-16 & 8.03 \mathrm{E}-15 & 7.91 \mathrm{E}-11 \\ 12 & 1.20 \mathrm{E}+03 & 2.57 \mathrm{E}-10 & 1.29 \mathrm{E}-08 & 1.27 \mathrm{E}-04 \\ 23 & 2.30 \mathrm{E}+03 & 2.33 \mathrm{E}-11 & 1.17 \mathrm{E}-09 & 1.15 \mathrm{E}-05 \\ 34 & 3.40 \mathrm{E}+03 & 9.76 \mathrm{E}-25 & 4.89 \mathrm{E}-23 & 4.81 \mathrm{E}-19\end{array}$

OUTPUT TABLE 2, Concentration and Flux data at Time $=2.0000 \mathrm{E}+00$

Mass Units are $\mathrm{Ci} \quad * \star \star \operatorname{ITIME}=2$

MIXING BATH MODEL PREDICTED CONCENTRATIONS AND FLUXES

$\begin{array}{rcccc}\text { LOC } & \text { DIST }(\mathrm{cm}) & \text { CONC Ci/cm^3 } & \text { FLUX Ci/cm^2/yr } & \text { FLOW RATE Ci/yr } \\ 1 & 1.00 \mathrm{E}+02 & 3.22 \mathrm{E}-09 & 1.61 \mathrm{E}-07 & 1.59 \mathrm{E}-03 \\ 7 & 7.00 \mathrm{E}+02 & 1.52 \mathrm{E}-14 & 7.63 \mathrm{E}-13 & 7.52 \mathrm{E}-09 \\ 12 & 1.20 \mathrm{E}+03 & 8.14 \mathrm{E}-10 & 4.07 \mathrm{E}-08 & 4.01 \mathrm{E}-04 \\ 23 & 2.30 \mathrm{E}+03 & 1.44 \mathrm{E}-10 & 7.20 \mathrm{E}-09 & 7.09 \mathrm{E}-05 \\ 34 & 3.40 \mathrm{E}+03 & 1.37 \mathrm{E}-23 & 6.84 \mathrm{E}-22 & 6.74 \mathrm{E}-18\end{array}$

OUTPUT TABLE 3, Concentration and Flux data at Time $=5.0000 \mathrm{E}+00$ Mass Units are $\mathrm{Ci} \quad \star \star \star \operatorname{ITIME}=3$

MIXING BATH MODEL PREDICTED CONCENTRATIONS AND FLUXES

$\begin{array}{rl}\text { LOC } & \text { DIST }(\mathrm{Cm} \\ 1 & 1.00 \mathrm{E}+02 \\ 7 & 7.00 \mathrm{E}+02 \\ 12 & 1.20 \mathrm{E}+03 \\ 23 & 2.30 \mathrm{E}+03 \\ 34 & 3.40 \mathrm{E}+03\end{array}$

$\mathrm{CONC} \mathrm{Ci} / \mathrm{cm}^{\wedge} 3$
$4.79 \mathrm{E}-09$
$3.83 \mathrm{E}-12$
$2.58 \mathrm{E}-09$
$1.05 \mathrm{E}-09$
$2.04 \mathrm{E}-18$

FLUX $\mathrm{Ci} / \mathrm{cm}^{\wedge} 2 / \mathrm{yr}$

$2.40 \mathrm{E}-07$
$1.92 \mathrm{E}-10$
$1.29 \mathrm{E}-07$
$5.25 \mathrm{E}-08$
$1.02 \mathrm{E}-16$

FLOW RATE Ci/yr

2.36E-03

$1.89 \mathrm{E}-06$

$1.27 \mathrm{E}-03$

$5.17 \mathrm{E}-04$

1. $01 \mathrm{E}-12$ 
At the top of this file are a number of cards which are used to define the problem. The format used on these cards is mandatory if the program GRAFXT is to be used. The first card in this file prints the title as defined by input. This is followed by a card providing the number of concentration traces and the units of concentration. This is followed by a blank card. Next is the list of the node numbers at which a trace is requested. These values are printed in columns such that the data underneath this label is the concentration at the specified node. The location of these nodes is printed on the next line. The last problem definition card prints the label TIME (YRS) over the first column of data and provides headings for the columns of concentration values.

The remainder of this file contains the time followed by the concentration at each of the trace locations. Up to 20 concentrations, the maximum number allowed by DUST, will be written on a single line. An example of a concentration trace file is presented in Table 8.3. The structure of this file is independent of the choice of transport model.

The file name TRACECND.DAT is used every time a trace file is requested by the DUST code. If the output of this file needs to be saved, the file must be renamed prior to using the DUST code.

\subsection{Flux Trace File (TRCFXD?.DAT)}

The flux trace file is also intended primarily for use with GRAFXT and other graphics programs. TRACEFXD contains the flux (units are mass $/ \mathrm{cm}^{2} / \mathrm{yr}$ with the mass in either grams or Curies depending on mass units specified through input), an estimate for the cumulative amount of mass that has passed the location as a function of time (flux integrated over time and area, units of mass), and the mass flow rate (flux integrated over the area, units of mass/yr).

The structure of the file is similar to TRACECND, however, because it contains three variables per trace location, there are important differences. The first card is the title card. The next card provides the definition of the number of trace locations and the units used for the output. This is followed by a blank card.

At this point, the structure differs from that used in TRACECND. The values for the node are printed three times, once for the flux, the integrated flux, and for the mass flow rate. Up to 7 nodes, 21 values, are printed per line. If more than 7 flux trace nodes are specified, additional lines are printed. GRAFXT automatically adjusts for this. However, if TRACEFXD is imported into a spreadsheet or other graphics program, adjustments to the file may be required.

Following the node numbers, the location of each node is printed. Again, the location is printed three times, once for each variable of interest. After the locations are printed, the label TIME (YRS) is printed on a separate line and labels (flux, Mass Rel for the mass release, and Mass Rate for the mass flow rate) are printed for each column. 
Table 8.3 Typical concentration trace file, TRACECND.DAT

FD MODEL $-\mathrm{H}-3, \mathrm{MC}=0.2, \mathrm{VDAR}=1.58 \mathrm{E}-6, \mathrm{MLT}$ CONT

NUMBER OF CONCENTRATION TRACES $=5$ Units are Curies per cubic centimeter

\begin{tabular}{|c|c|c|c|c|c|}
\hline NODE & 11 & 17 & 22 & 33 & \\
\hline ISTANCE & $1.00 \mathrm{E}+03$ & $1.60 \mathrm{E}+03$ & $2.10 \mathrm{E}+03$ & $3.20 \mathrm{E}+03$ & $4.30 \mathrm{E}+03$ \\
\hline $\mathrm{ME}$ (YRS) & Conc & Conc & Conc & Conc & Conc \\
\hline $00 \mathrm{E}+00$ & $0.00 \mathrm{E}+00$ & $0.00 \mathrm{E}+00$ & $0.00 \mathrm{E}+00$ & $0.00 \mathrm{E}+00$ & $0.00 \mathrm{E}+00$ \\
\hline $0 O E+00$ & $2.90 \mathrm{E}-09$ & $8.71 \mathrm{E}-13$ & $8.73 E-10$ & $2.38 \mathrm{E}-10$ & $2.76 \mathrm{E}-17$ \\
\hline 00 & $22 \mathrm{E}-09$ & $3 \mathrm{E}-12$ & & $63 E-10$ & \\
\hline+00 & $4.69 \mathrm{E}-09$ & $2 \mathrm{E}-11$ & $78 \mathrm{E}-09$ & $38 \mathrm{E}-09$ & $.24 \mathrm{E}-1$ \\
\hline $0 E+00$ & $4.70 \mathrm{E}-09$ & $8.17 \mathrm{E}-11$ & $3.28 \mathrm{E}-09$ & $1.94 \mathrm{E}-09$ & 4. $22 \mathrm{E}-1$ \\
\hline $00 E+01$ & $4.49 \mathrm{E}-09$ & $1.65 \mathrm{E}-10$ & $3.50 E-09$ & $2.36 \mathrm{E}-09$ & $1.73 \mathrm{E}-13$ \\
\hline 01 & $4.18 \mathrm{E}-09$ & $2.89 \mathrm{E}-10$ & $3.52 \mathrm{E}-09$ & $2.61 \mathrm{E}-09$ & $5.52 \mathrm{E}-13$ \\
\hline & $3.82 \mathrm{E}-09$ & $4.67 \mathrm{E}-10$ & $3.41 E-09$ & $2.72 \mathrm{E}-09$ & 1. $45 \mathrm{E}-1$ \\
\hline & $3.47 \mathrm{E}-09$ & 7.02E-10 & $3.22 \mathrm{E}-09$ & $2.72 \mathrm{E}-09$ & $30 \mathrm{E}-1$ \\
\hline & $3.13 \mathrm{E}-09$ & $9.77 \mathrm{E}-10$ & $3.00 \mathrm{E}-09$ & $2.64 \mathrm{E}-09$ & $6.61 \mathrm{E}-12$ \\
\hline+01 & $2.81 \mathrm{E}-09$ & $1.26 \mathrm{E}-09$ & $2.77 \mathrm{E}-09$ & $2.53 \mathrm{E}-09$ & $1.20 \mathrm{E}-11$ \\
\hline 01 & $1.58 \mathrm{E}-09$ & $1.60 \mathrm{E}-09$ & $2.27 \mathrm{E}-09$ & $2.32 \mathrm{E}-09$ & $2.00 \mathrm{E}-11$ \\
\hline 01 & $8.87 \mathrm{E}-10$ & $1.98 \mathrm{E}-09$ & $1.73 E-09$ & $2.04 \mathrm{E}-09$ & $3.11 \mathrm{E}-1$ \\
\hline & $4.98 \mathrm{E}-10$ & $2.33 \mathrm{E}-09$ & 1. $30 \mathrm{E}-09$ & $1.78 \mathrm{E}-$ & $54 \mathrm{E}-1$ \\
\hline & $2.80 \mathrm{E}-10$ & $2.59 \mathrm{E}-09$ & $9.98 \mathrm{E}-10$ & $1.60 \mathrm{E}-09$ & $6.28 \mathrm{E}-1$ \\
\hline 01 & $1.57 \mathrm{E}-10$ & $2.74 \mathrm{E}-09$ & $8.22 \mathrm{E}-10$ & $1.50 \mathrm{E}-09$ & $8.29 \mathrm{E}-1$ \\
\hline 01 & $8.82 \mathrm{E}-11$ & $3.30 \mathrm{E}-09$ & 7.40 & $2.03 \mathrm{E}-$ & 1. $05 \mathrm{E}-1$ \\
\hline 01 & $4.95 \mathrm{E}-11$ & $3.47 \mathrm{E}-09$ & $7.26 \mathrm{E}-10$ & 2.31 & 1.28E-1 \\
\hline 01 & $2.78 \mathrm{E}-11$ & $3.39 \mathrm{E}-09$ & $7.59 \mathrm{E}-10$ & $2.48 \mathrm{E}-09$ & $1.51 \mathrm{E}-1$ \\
\hline & $1.56 \mathrm{E}-11$ & $3.17 \mathrm{E}-09$ & $8.21 \mathrm{E}-10$ & $2.56 \mathrm{E}-09$ & $1.73 \mathrm{E}-1$ \\
\hline & $8.77 \mathrm{E}-12$ & $2.87 \mathrm{E}-09$ & $8.95 E-10$ & $2.59 \mathrm{E}-$ & $1.93 \mathrm{E}-1$ \\
\hline 01 & $4.92 \mathrm{E}-12$ & $2.52 \mathrm{E}-09$ & $9.75 \mathrm{E}-10$ & 2.57 & $2.11 \mathrm{E}-1$ \\
\hline & $2.76 \mathrm{E}-12$ & $2.15 \mathrm{E}-09$ & $1.06 \mathrm{E}-$ & $2.51 \mathrm{E}$ & $2.26 \mathrm{E}-1$ \\
\hline+01 & $1.55 \mathrm{E}-12$ & $1.78 \mathrm{E}-09$ & $1.13 \mathrm{E}-09$ & $2.40 \mathrm{E}-09$ & $2.38 \mathrm{E}-1$ \\
\hline & $8.72 \mathrm{E}-13$ & $1.45 \mathrm{E}-09$ & $1.19 \mathrm{E}-09$ & $2.26 \mathrm{E}-09$ & $2.48 E-1$ \\
\hline & $4.90 \mathrm{E}-13$ & $1.17 \mathrm{~F}$ & 1.22 & 2.08 & $2.56 \mathrm{E}-1$ \\
\hline & $2.75 \mathrm{E}-13$ & $7.71 \mathrm{E}-10$ & $1.22 \mathrm{E}-09$ & $1.72 \mathrm{E}-09$ & $2.63 \mathrm{E}-1$ \\
\hline & $1.54 \mathrm{E}-13$ & $5.09 \mathrm{E}-10$ & $1.18 \mathrm{E}-09$ & $1.44 \mathrm{E}-09$ & $2.68 \mathrm{E}-1$ \\
\hline & $8.67 \mathrm{E}-14$ & $3.36 \mathrm{E}-10$ & $1.12 \mathrm{E}-$ & $1.21 \mathrm{~F}$ & $2.74 \mathrm{E}-1$ \\
\hline & $4.87 E-14$ & $2.23 \mathrm{E}$ & $1.04 \mathrm{E}-$ & 1.0 & $2.78 \mathrm{E}-1$ \\
\hline & $2.73 \mathrm{E}-14$ & $1.47 \mathrm{E}$ & 9.41 & 8.5 & $2.83 E-1$ \\
\hline & $1.54 \mathrm{E}-14$ & $9.74 \mathrm{E}-11$ & $8.32 \mathrm{E}-1$ & 7. $20 \mathrm{E}-1$ & $2.86 \mathrm{E}-1$ \\
\hline & $8.62 \mathrm{E}-15$ & $6.43 \mathrm{E}-11$ & $7.17 \mathrm{E}-$ & $6.04 \mathrm{E}-$ & $2.89 \mathrm{E}-1$ \\
\hline & $4.84 \mathrm{E}-15$ & $4.23 E-11$ & $6.03 E-10$ & $5.07 \mathrm{E}-10$ & $2.91 \mathrm{E}-1$ \\
\hline & $2.72 \mathrm{E}-15$ & $2.78 \mathrm{E}$ & & 4.28 & $2.91 \mathrm{E}-1$ \\
\hline & $1.53 \mathrm{E}-15$ & $1.82 \mathrm{E}-11$ & 3.9 & 3.641 & $2.88 \mathrm{E}-1$ \\
\hline & $8.57 \mathrm{E}-16$ & $1.19 \mathrm{E}-11$ & $3.10 \mathrm{E}-1$ & $3.12 \mathrm{E}-$ & $2.84 \mathrm{E}-1$ \\
\hline & $4.81 \mathrm{E}-16$ & 7. $78 \mathrm{E}-12$ & $2.39 \mathrm{E}-$ & $2.72 \mathrm{E}-$ & $2.77 \mathrm{E}-1$ \\
\hline & $2.70 \mathrm{E}-16$ & $5.06 \mathrm{E}-12$ & $1.81 \mathrm{E}-10$ & $2.39 \mathrm{E}-10$ & $2.67 \mathrm{E}-1$ \\
\hline & $1.52 \mathrm{E}-16$ & $3.28 \mathrm{E}-12$ & 1. $35 \mathrm{E}-10$ & $2.14 \mathrm{E}-$ & $2.56 \mathrm{E}-1$ \\
\hline & $8.53 \mathrm{E}-17$ & $2.12 \mathrm{E}-12$ & $9.97 \mathrm{E}-$ & $1.93 \mathrm{E}-$ & $2.42 \mathrm{E}-1$ \\
\hline & $4.79 \mathrm{E}-17$ & 1. $37 \mathrm{E}-1$ & $7.28 \mathrm{E}-$ & $1.76 \mathrm{E}-$ & $2.27 \mathrm{E}-1$ \\
\hline & $2.69 \mathrm{E}-17$ & $8.82 \mathrm{E}-13$ & $5.27 \mathrm{E}-11$ & $1.61 \mathrm{E}-10$ & $2.11 \mathrm{E}-1$ \\
\hline & $1.51 \mathrm{E}-17$ & $5.66 \mathrm{E}-13$ & $3.78 \mathrm{E}-11$ & $1.48 \mathrm{E}-10$ & $1.94 \mathrm{E}-1$ \\
\hline & $8.48 \mathrm{E}-18$ & $3.62 \mathrm{E}-13$ & $2.69 \mathrm{E}-11$ & $1.37 \mathrm{E}-10$ & \\
\hline & . & $232 \mathrm{~F}-1$ & 1. $90 \mathrm{E}-1$ & $1.26 \mathrm{E}-10$ & \\
\hline
\end{tabular}


Table 8.4 Typical flux trace file, TRACEFXD.DAT

FD MODEL $-\mathrm{H}-3, \mathrm{MC}=0.2, \mathrm{VDAR}=1.58 \mathrm{E}-6, \mathrm{MLT}$ CONT

NUMBER OF FLUX TRACES = 5 Flux units are Curies per $\mathrm{cm}^{\wedge} 2 / \mathrm{yr}^{\circ}$

\begin{tabular}{|c|c|c|c|c|c|c|}
\hline NODE & 11 & 11 & 11 & 17 & 17 & 17 \\
\hline DISTANCE & $1.00 \mathrm{E}+03$ & $1.00 \mathrm{E}+03$ & $1.00 \mathrm{E}+03$ & $1.60 \mathrm{E}+03$ & $1.60 \mathrm{E}+03$ & $1.60 \mathrm{E}+03$ \\
\hline ME (YRS) & Flux & Mass Rel & Mass Rate & Flux & Mass $\operatorname{Rel}$ & Mass Rate \\
\hline $00 E+00$ & $0.00 \mathrm{E}+00$ & $0.00 \mathrm{E}+00$ & $0.00 \mathrm{E}+00$ & $0.00 \mathrm{E}+00$ & $0.00 \mathrm{E}+00$ & $0.00 \mathrm{E}+00$ \\
\hline $00 E+00$ & 1. $45 \mathrm{E}-07$ & $2.28 \mathrm{E}-03$ & $1.43 \mathrm{E}-03$ & $4.36 \mathrm{E}-11$ & $4.99 E-07$ & $4.30 \mathrm{E}-07$ \\
\hline $.00 E+00$ & $2.12 \mathrm{E}-07$ & $6.19 \mathrm{E}-03$ & $2.08 \mathrm{E}-03$ & $3.93 E-10$ & $5.87 \mathrm{E}-06$ & $3.87 \mathrm{E}-06$ \\
\hline$E+00$ & $2.35 E-07$ & $1.07 \mathrm{E}-02$ & $2.31 \mathrm{E}-03$ & $1.56 \mathrm{E}-09$ & $2.95 E-05$ & $1.54 \mathrm{E}-05$ \\
\hline $00 E+00$ & $2.36 \mathrm{E}-07$ & $1.54 \mathrm{E}-02$ & $2.32 \mathrm{E}-03$ & $4.09 \mathrm{E}-09$ & $9.57 \mathrm{E}-05$ & $4.03 E-05$ \\
\hline $00 E+01$ & $2.25 E-07$ & $1.99 \mathrm{E}-02$ & $2.22 \mathrm{E}-03$ & $8.27 \mathrm{E}-09$ & $2.36 \mathrm{E}-04$ & $8.15 \mathrm{E}-05$ \\
\hline $20 E+01$ & $2.09 E-07$ & $2.41 \mathrm{E}-02$ & $2.06 \mathrm{E}-03$ & $1.45 \mathrm{E}-08$ & $4.87 \mathrm{E}-04$ & $1.42 \mathrm{E}-04$ \\
\hline $40 E+01$ & $1.92 \mathrm{E}-07$ & $2.80 E-02$ & $1.89 \mathrm{E}-03$ & $2.34 \mathrm{E}-08$ & $9.00 E-04$ & $2.30 E-04$ \\
\hline & $1.74 \mathrm{E}-07$ & $3.15 E-02$ & $1.71 \mathrm{E}-03$ & $3.52 \mathrm{E}-08$ & $1.53 E-03$ & $3.46 \mathrm{E}-04$ \\
\hline $80 E+01$ & $1.57 \mathrm{E}-07$ & $3.46 \mathrm{E}-02$ & $1.54 \mathrm{E}-03$ & $4.89 E-08$ & $2.43 E-03$ & $4.82 \mathrm{E}-04$ \\
\hline $00 E+01$ & $1.41 \mathrm{E}-07$ & $3.75 E-02$ & $1.39 \mathrm{E}-03$ & $6.31 \mathrm{E}-08$ & $3.60 \mathrm{E}-03$ & $6.22 \mathrm{E}-04$ \\
\hline $20 E+01$ & $7.91 \mathrm{E}-08$ & $3.93 \mathrm{E}-02$ & $7.79 \mathrm{E}-04$ & $8.01 \mathrm{E}-08$ & $5.09 \mathrm{E}-03$ & $7.89 \mathrm{E}-04$ \\
\hline $\mathrm{E}+01$ & $4.44 \mathrm{E}-08$ & $4.03 E-02$ & $4.37 \mathrm{E}-04$ & $9.93 E-08$ & $6.95 \mathrm{E}-03$ & $9.78 \mathrm{E}-04$ \\
\hline $60 \mathrm{E}+01$ & $2.49 \mathrm{E}-08$ & $4.09 \mathrm{E}-02$ & $2.46 \mathrm{E}-04$ & $1.17 \mathrm{E}-07$ & $9.17 \mathrm{E}-03$ & $1.15 \mathrm{E}-03$ \\
\hline $80 E+01$ & 1. $40 \mathrm{E}-08$ & $4.12 \mathrm{E}-02$ & $1.38 \mathrm{E}-04$ & $1.30 \mathrm{E}-07$ & $1.17 \mathrm{E}-02$ & $1.28 \mathrm{E}-03$ \\
\hline $00 \mathrm{E}+01$ & $7.86 \mathrm{E}-09$ & $4.14 \mathrm{E}-02$ & $7.75 \mathrm{E}-05$ & $1.37 \mathrm{E}-07$ & $1.43 E-02$ & $1.35 \mathrm{E}-03$ \\
\hline $20 E+01$ & 4. $42 \mathrm{E}-09$ & $4.15 \mathrm{E}-02$ & $4.35 \mathrm{E}-05$ & $1.65 \mathrm{E}-07$ & $1.75 \mathrm{E}-02$ & $1.63 \mathrm{E}-03$ \\
\hline $40 E+01$ & $2.48 E-09$ & $4.16 \mathrm{E}-02$ & $2.44 \mathrm{E}-05$ & $1.74 \mathrm{E}-07$ & $2.09 \mathrm{E}-02$ & $1.71 \mathrm{E}-03$ \\
\hline $\mathrm{E}+01$ & 1. $39 \mathrm{E}-09$ & $4.16 \mathrm{E}-02$ & $1.37 \mathrm{E}-05$ & $1.70 \mathrm{E}-07$ & $2.43 E-02$ & $1.67 \mathrm{E}-03$ \\
\hline$E+01$ & 7. $82 \mathrm{E}-10$ & $4.16 \mathrm{E}-02$ & $7.70 \mathrm{E}-06$ & $1.59 \mathrm{E}-07$ & $2.75 E-02$ & $1.56 \mathrm{E}-03$ \\
\hline$E+01$ & $4.39 \mathrm{E}-10$ & $4.16 \mathrm{E}-02$ & $4.33 \mathrm{E}-06$ & 1. $44 \mathrm{E}-07$ & $3.04 \mathrm{E}-02$ & $1.42 \mathrm{E}-03$ \\
\hline $20 E+01$ & $2.47 \mathrm{E}-10$ & $4.16 \mathrm{E}-02$ & $2.43 E-06$ & $1.26 \mathrm{E}-07$ & $3.29 \mathrm{E}-02$ & $1.24 \mathrm{E}-03$ \\
\hline $40 E+01$ & 1. $38 \mathrm{E}-10$ & $4.16 \mathrm{E}-02$ & $1.36 \mathrm{E}-06$ & $1.08 \mathrm{E}-07$ & $3.51 E-02$ & $1.06 \mathrm{E}-03$ \\
\hline$E+01$ & $7.78 \mathrm{E}-11$ & $4.16 \mathrm{E}-02$ & $7.66 \mathrm{E}-07$ & $8.92 \mathrm{E}-08$ & $3.70 \mathrm{E}-02$ & $8.79 \mathrm{E}-04$ \\
\hline $80 \mathrm{E}+01$ & $4.37 \mathrm{E}-11$ & $4.16 \mathrm{E}-02$ & $4.30 \mathrm{E}-07$ & $7.28 \mathrm{E}-08$ & $3.85 E-02$ & $7.17 \mathrm{E}-04$ \\
\hline $.00 E+01$ & $2.45 \mathrm{E}-11$ & $4.16 \mathrm{E}-02$ & $2.42 \mathrm{E}-07$ & $5.88 \mathrm{E}-08$ & $3.97 \mathrm{E}-02$ & $5.79 \mathrm{E}-04$ \\
\hline $20 E+01$ & 1. $38 \mathrm{E}-11$ & $4.16 \mathrm{E}-02$ & $1.36 \mathrm{E}-07$ & $3.86 \mathrm{E}-08$ & $4.06 \mathrm{E}-02$ & $3.80 \mathrm{E}-04$ \\
\hline+01 & 7. $73 \mathrm{E}-12$ & $4.16 \mathrm{E}-02$ & $7.62 \mathrm{E}-08$ & $2.55 \mathrm{E}-08$ & $4.11 E-02$ & $2.51 \mathrm{E}-04$ \\
\hline$E+01$ & $4.34 E-12$ & $4.16 \mathrm{E}-02$ & $4.28 E-08$ & $1.68 \mathrm{E}-08$ & $4.15 E-02$ & $1.66 \mathrm{E}-04$ \\
\hline $\mathrm{E}+01$ & $2.44 \mathrm{E}-12$ & $4.16 \mathrm{E}-02$ & $2.40 \mathrm{E}-08$ & $1.11 \mathrm{E}-08$ & $4.18 \mathrm{E}-02$ & $1.10 \mathrm{E}-04$ \\
\hline$E+01$ & 1. $37 \mathrm{E}-12$ & $4.16 \mathrm{E}-02$ & 1. $35 \mathrm{E}-08$ & $7.38 \mathrm{E}-09$ & $4.19 \mathrm{E}-02$ & $7.26 \mathrm{E}-05$ \\
\hline$E+01$ & 7. $69 \mathrm{E}-13$ & $4.16 \mathrm{E}-02$ & $7.57 \mathrm{E}-09$ & $4.88 \mathrm{E}-09$ & $4.20 E-02$ & $4.80 \mathrm{E}-05$ \\
\hline $40 E+01$ & 4. $32 \mathrm{E}-13$ & $4.16 \mathrm{E}-02$ & $4.25 \mathrm{E}-09$ & $3.22 \mathrm{E}-09$ & $4.21 \mathrm{E}-02$ & $3.17 \mathrm{E}-05$ \\
\hline$D E+01$ & $2.42 \mathrm{E}-13$ & $4.16 \mathrm{E}-02$ & $2.39 \mathrm{E}-09$ & $2.12 \mathrm{E}-09$ & $4.21 E-02$ & $2.09 \mathrm{E}-05$ \\
\hline$E+01$ & 1. $36 \mathrm{E}-13$ & $4.16 \mathrm{E}-02$ & $1.34 \mathrm{E}-09$ & 1. $39 \mathrm{E}-09$ & $4.22 E-02$ & $1.37 \mathrm{E}-05$ \\
\hline & 7. $65 \mathrm{E}-14$ & $4.16 \mathrm{E}-02$ & $7.53 \mathrm{E}-10$ & $9.14 \mathrm{E}-10$ & $4.22 E-02$ & $9.00 \mathrm{E}-06$ \\
\hline$E+01$ & $4.29 \mathrm{E}-14$ & $4.16 \mathrm{E}-02$ & $4.23 \mathrm{E}-10$ & $5.98 \mathrm{E}-10$ & $4.22 \mathrm{E}-02$ & $5.89 \mathrm{E}-06$ \\
\hline $40 E+01$ & $2.41 E-14$ & $4.16 \mathrm{E}-02$ & $2.38 \mathrm{E}-10$ & $3.90 \mathrm{E}-10$ & $4.22 \mathrm{E}-02$ & $3.84 \mathrm{E}-06$ \\
\hline $0 E+01$ & 1. $35 E-14$ & $4.16 \mathrm{E}-02$ & 1. $33 \mathrm{E}-10$ & $2.53 E-10$ & $4.22 E-02$ & $2.50 \mathrm{E}-06$ \\
\hline$D E+01$ & 7. $60 \mathrm{E}-15$ & $4.16 \mathrm{E}-02$ & 7. $49 \mathrm{E}-11$ & 1. $64 \mathrm{E}-10$ & $4.22 E-02$ & $1.62 \mathrm{E}-06$ \\
\hline & $4.27 E-15$ & $4.16 \mathrm{E}-02$ & $4.21 \mathrm{E}-11$ & $1.06 \mathrm{E}-10$ & $4.22 E-02$ & $1.05 \mathrm{E}-06$ \\
\hline & $2.40 E-15$ & $4.16 \mathrm{E}-02$ & $2.36 \mathrm{E}-11$ & $6.86 \mathrm{E}-11$ & $4.22 E-02$ & $6.76 \mathrm{E}-07$ \\
\hline & 1. $35 \mathrm{E}-15$ & $4.16 \mathrm{E}-02$ & 1. $33 \mathrm{E}-11$ & 4. $42 \mathrm{E}-11$ & $4.22 \mathrm{E}-02$ & $4.35 \mathrm{E}-07$ \\
\hline 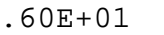 & $7.56 \mathrm{E}-16$ & $4.16 \mathrm{E}-02$ & 7. $45 \mathrm{E}-12$ & $2.83 E-11$ & $4.22 \mathrm{E}-02$ & $2.79 \mathrm{E}-07$ \\
\hline & $4.25 E-16$ & $4.16 \mathrm{E}-02$ & $4.18 \mathrm{E}-12$ & $1.82 \mathrm{E}-11$ & $4.22 \mathrm{E}-02$ & $1.79 \mathrm{E}-07$ \\
\hline $0 E+01$ & $2.38 \mathrm{E}-16$ & $4.16 \mathrm{E}-02$ & $2.35 \mathrm{E}-12$ & $1.16 \mathrm{E}-11$ & $4.22 \mathrm{E}-02$ & $1.14 \mathrm{E}-07$ \\
\hline
\end{tabular}


The remainder of the file contains a series of columns of values. The first column is the time, the second column is the flux at the first trace location, the third column is the integrated flux (units of mass, either Curies or grams depending on the input), and the fourth column is the mass flow rate (units of mass per year with the mass units in Curies or grams depending on the input). The three values (flux, integrated flux, and mass flow rate), are repeated for each trace location. If more than 7 trace locations are specified, additional cards are needed to define the output at each time. An example of a flux trace file is presented in Table 8.4. Although, the example is for the FD transport model, the structure is identical if the MCMC model was used.

The file name TRACEFXD.DAT is used every time a trace file is requested by the DUST code. If the output of this file needs to be saved, the file must be renamed prior to reuse of the DUST code.

\subsection{Concentration File (CONCNT.DAT)}

When the FD transport model is used, every time the print flag KPR is greater than zero, the concentration at every location is written to the primary output file and to CONCNT.DAT. The file CONCNT.DAT complements the file TRACECND.DAT. Typically, CONCNT.DAT will have the concentrations at every location at a few specified times while TRACECND.DAT will have the concentrations at a few locations at almost every time step.

The first line in CONCNT.DAT contains the title specified by the code user. The second line defines the number of locations and the units for concentration in the output file. These are followed by the value for the locations beginning with the first node. In CONCNT.DAT, since the number of locations will vary between different problems, the WRITE statements print out seven values per line. The number of lines printed depends on the number of values. In the example provided in Table 8.5, there are 50 locations. Therefore, there are 8 lines containing locations (seven with seven values and the last with one value). After the locations are printed, a line containing the problem time at which the concentration values were written is displayed. This is followed by the values for the concentration at each node. Again, these are written seven to a line beginning with Node 1.

Each time output is requested, the sequence of lines containing the time followed by the concentration values is repeated, Table 8.5.

As this file stands, it is not in a form that is useful for importing into spreadsheets or other graphics packages. To overcome this limitation, the program IOSWTCH was written. IOSWTCH reads and reformats the file CONCNT.DAT. It creates a new file CONCNT2.DAT that contains the title card followed by a card specifying the number of locations. This is followed by a series of rows and columns of primarily numerical data. The first row contains the alphanumeric string "TIME/LOC" in column 1 while columns 2 through the end contain the output time. The second row contains the location in column 1 and the values for concentration at the various time in columns 2 through the end. That is, the output file can be viewed as a matrix containing columns of 
FD MODEL $-\mathrm{H}-3, \mathrm{MC}=0.2, \mathrm{VDAR}=1.58 \mathrm{E}-6, \mathrm{MLT}$ CONT $0.00 \mathrm{E}+00 \quad 1.00 \mathrm{E}+02$

$7.00 \mathrm{E}+02 \quad 8.00 \mathrm{E}+02$
$1.40 \mathrm{E}+03$

1. $40 \mathrm{E}+03 \quad 1.50 \mathrm{E}+03$

$2.10 \mathrm{E}+03 \quad 2.20 \mathrm{E}+03$

$2.80 \mathrm{E}+03 \quad 2.90 \mathrm{E}+03$

$3.50 \mathrm{E}+03 \quad 3.60 \mathrm{E}+03$

$4.20 \mathrm{E}+03 \quad 4.30 \mathrm{E}+03$

Concentration units are in Curies per $\mathrm{Cm}^{\wedge} 3$

$\begin{array}{llll} & 3.00 \mathrm{E}+02 & 4.00 \mathrm{E}+02 & 5.00 \mathrm{E}+02\end{array}$

$9.00 \mathrm{E}+02 \quad 1.00 \mathrm{E}+03$

$1.60 \mathrm{E}+03 \quad 1.70 \mathrm{E}+03$

2. $30 \mathrm{E}+03 \quad 2.40 \mathrm{E}+03$

$3.00 \mathrm{E}+03 \quad 3.10 \mathrm{E}+03$

$1.10 \mathrm{E}+03$

$1.20 \mathrm{E}+03$

$1.80 \mathrm{E}+03 \quad 1.90 \mathrm{E}+03$

$2.50 \mathrm{E}+03 \quad 2.60 \mathrm{E}+03$

$3.70 \mathrm{E}+03 \quad 3.80 \mathrm{E}+03$

$3.20 \mathrm{E}+03$

3. $30 \mathrm{E}+03$

$3.90 \mathrm{E}+03 \quad 4.00 \mathrm{E}+03$

$4.40 \mathrm{E}+03 \quad 4.50 \mathrm{E}+03$

$4.60 \mathrm{E}+03$

4. $70 \mathrm{E}+03$

$6.00 \mathrm{E}+02$

1. $30 \mathrm{E}+03$

2. $00 \mathrm{E}+03$

2. $70 \mathrm{E}+03$

3. $40 \mathrm{E}+03$

$4.10 \mathrm{E}+03$

4. $90 \mathrm{E}+03$

TIME (yrs) $=1.00 \mathrm{E}+00$

$0.00 \mathrm{E}+00$

$0.00 \mathrm{E}+00$

$0.00 \mathrm{E}+00$

3. $24 \mathrm{E}-12$

$0.00 \mathrm{E}+00$

$0.00 \mathrm{E}+00$

$0.00 \mathrm{E}+00$

$0.00 \mathrm{E}+00$

$1.71 \mathrm{E}-09$

$6.75 \mathrm{E}-13$

1. $41 \mathrm{E}-13$

2. $93 \mathrm{E}-14$

1. $55 \mathrm{E}-11$

3. $24 \mathrm{E}-12$

$3.57 \mathrm{E}-10$
$6.12 \mathrm{E}-15$

$7.44 \mathrm{E}-11$

1. $71 \mathrm{E}-09$

3. $57 \mathrm{E}-10$

$1.28 \mathrm{E}-15$

2. $93 \mathrm{E}-14$

6.
$1.16 \mathrm{E}-17$

2. $41 \mathrm{E}-18$

5. $03 \mathrm{E}-19$

$6.12 \mathrm{E}-15$

1. $05 \mathrm{E}-19$

$0.00 \mathrm{E}+00$

3. $57 \mathrm{E}-10$

$0.00 \mathrm{E}+00$

$6.12 \mathrm{E}-15$

$7.44 \mathrm{E}-11$

$1.28 \mathrm{E}-15$

1. $41 \mathrm{E}-13$

7. $44 \mathrm{E}-11 \quad 1.55 \mathrm{E}-11$

1. $28 \mathrm{E}-15 \quad 2.66 \mathrm{E}-16$

$2.18 \mathrm{E}-20$

$4.56 \mathrm{E}-21$

4. $80 \mathrm{E}+03$

3. $28 \mathrm{E}-22$

TIME (yrs) $=2.00 \mathrm{E}+00$

$0.00 \mathrm{E}+00$

$=\quad 2.00 \mathrm{E}+00$
$0.00 \mathrm{E}+00$

$0.00 \mathrm{E}+00$

$0.00 \mathrm{E}+00$

$0.00 \mathrm{E}+00$

$0.00 \mathrm{E}+00$

$0.00 \mathrm{E}+00$

$0.00 \mathrm{E}+00$

$2.90 \mathrm{E}-09$

8. $71 \mathrm{E}-13$

$2.04 \mathrm{E}-13$

2. $38 \mathrm{E}-10$

8. $73 \mathrm{E}-10$

$1.08 \mathrm{E}-14$

$6.12 \mathrm{E}-11$

1. $52 \mathrm{E}-11$

2. $90 \mathrm{E}-09$

8. $73 \mathrm{E}-10$

$8.71 \mathrm{E}-13$

2. $04 \mathrm{E}-13$

$4.70 \mathrm{E}-14$

3. $67 \mathrm{E}-12$

2. $76 \mathrm{E}-17$

$6.12 \mathrm{E}-18$

1. $36 \mathrm{E}-18$

$8.73 \mathrm{E}-10$

4. $70 \mathrm{E}-14$

3. $67 \mathrm{E}-12$

2. $38 \mathrm{E}-10$

$1.08 \mathrm{E}-14$

2. $99 \mathrm{E}-19$

$0.00 \mathrm{E}+00$

2. $38 \mathrm{E}-10$

$1.08 \mathrm{E}-14$

8. $71 \mathrm{E}-13$

$6.12 \mathrm{E}-11$

$2.44 \mathrm{E}-15$

$6.57 \mathrm{E}-20$

$0.00 \mathrm{E}+00$

1. $55 \mathrm{E}-11$

1. $71 \mathrm{E}-09$

2. $93 \mathrm{E}-14$

3. $24 \mathrm{E}-12$

5. $54 \mathrm{E}-17$

9. $50 \mathrm{E}-22$

5. $18 \mathrm{E}-21$

TIME (yrs) $=5.00 \mathrm{E}+00$

$0.00 \mathrm{E}+00$

$0.00 \mathrm{E}+00$

$0.00 \mathrm{E}+00$

$0.00 \mathrm{E}+00$

$0.00 \mathrm{E}+00$

$0.00 E+00$

$2.41 \mathrm{E}-09$

1. $07 \mathrm{E}-09$

4. $53 \mathrm{E}-09$
5. $16 \mathrm{E}-12$

1. $53 \mathrm{E}-12$

4. $43 \mathrm{E}-13$

1. $54 \mathrm{E}-10$

$1.54 \mathrm{E}-10$

5. 21E-11

1. $67 \mathrm{E}-11$

4. $53 \mathrm{E}-09$

$5.21 \mathrm{E}-11$

1. $67 \mathrm{E}-11$

4. $43 \mathrm{E}-13$

1. $67 \mathrm{E}-11$

5. $16 \mathrm{E}-12$

1. $53 \mathrm{E}-12$

5. $21 \mathrm{E}-11$

2. $50 \mathrm{E}-15$

$6.56 \mathrm{E}-16$

1. $70 \mathrm{E}-16$

4. $24 \mathrm{E}-10$

4. $43 \mathrm{E}-13$ 1.25E-13

4.37E-17 1.11E-17

$0.00 \mathrm{E}+00$

$6.12 \mathrm{E}-11$

2. $90 \mathrm{E}-09$

2. $04 \mathrm{E}-13$

1. $52 \mathrm{E}-11$

5. 51E-16

1. $44 \mathrm{E}-20$

1. $12 \mathrm{E}-18$ 
concentration versus location at fixed times (or alternatively, rows of concentration versus time at fixed locations).

Both CONCNT.DAT and CONCNT2.DAT are default file names which are overwritten by the programs that create them. Therefore, if the user desires to save the output, these files need to be renamed.

\subsection{Wasteform Release Data (LEACHRL.DAT)}

If the FD transport model is used, the file LEACHRL.DAT is written at every time step that a trace is performed. This file contains the total mass released by release mechanism (rinse, diffusion, or uniform) and the release rate for each mechanism. The ratio of the breached area to total area as well as the location of the wasteform are also printed. The file contains three label cards. The first identifies this file as the wasteform release file. The second and third label cards provide information to identify the columns, Table 8.6.

This file contains the following 11 columns:

\section{Column:}

1) Time (yrs), the problem time at which the data are written.

2) Cumulative, cumulative mass release from all mechanisms for this wasteform.

3) Rinse, cumulative mass release from the rinse model.

4) Diffusion, cumulative mass release from the diffusion model.

5) Uniform, cumulative mass release from the uniform release model.

6) Total, total release rate from all mechanisms.

7) Rinse, rinse release rate.

8) Diffusion, diffusion release rate.

9) Uniform, uniform release rate.

10) Breach Ratio, ratio of breached container area to total area. A value of 1 indicates total failure of the container.

11) Node, location of the wasteform.

Columns 1 - 11 are written once for each wasteform at each time step. This file often becomes quite large when information is written at every time step.

This file is written every time the DUST code uses the FD transport model. If this information needs to be saved, the file must be renamed upon completion of DUST.

The example in Table 8.6 is from the test problem discussed in Section 8.1. After three years, only containers in control volumes 11, 21, and 31 have failed. All other containers are unbreached and therefore have zero release. Also, the only release mechanism modeled is uniform release. 


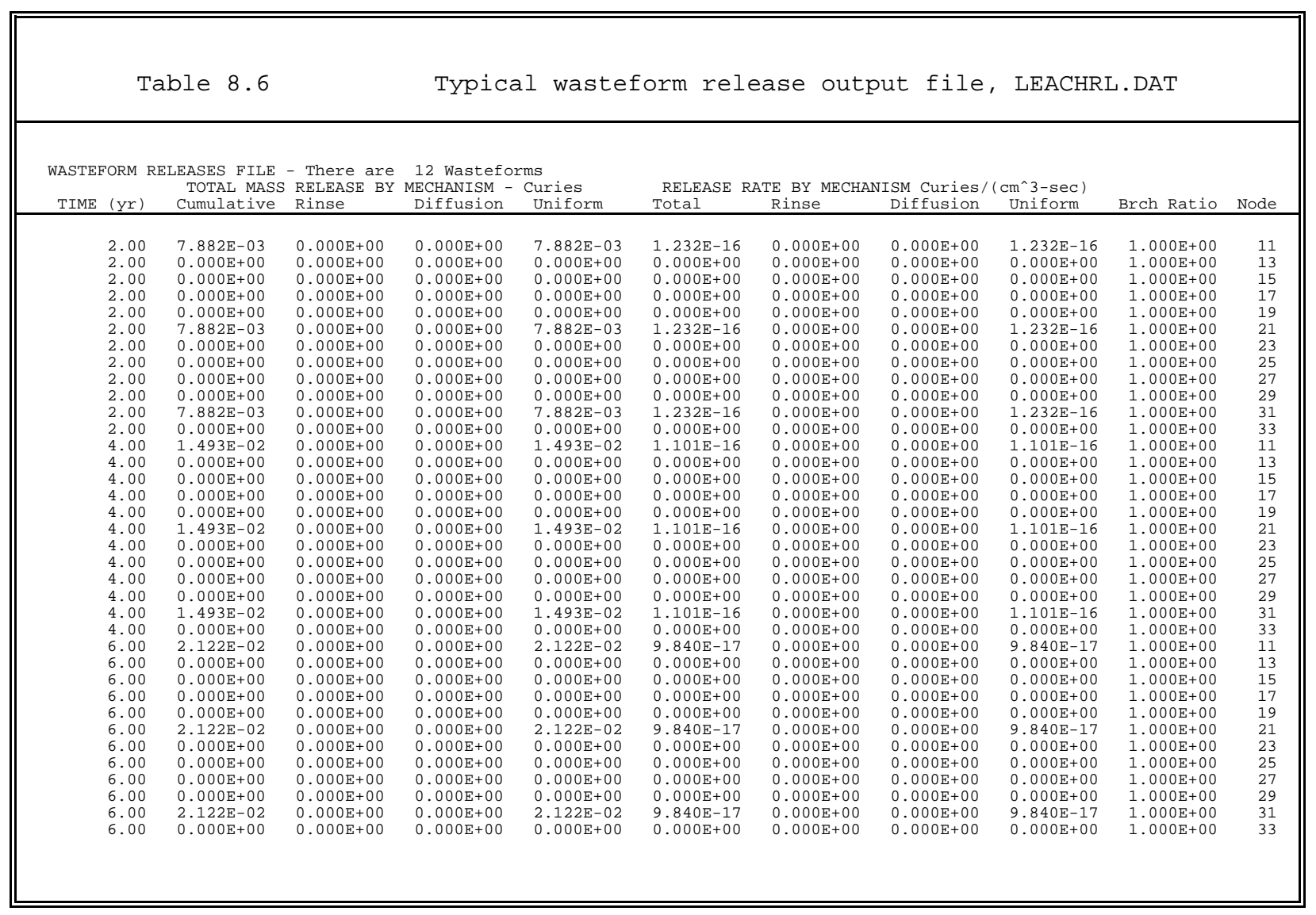

\subsection{Total Mass Release from the Wasteform}

The LEACHTMS.DAT file contains the total mass release from each wasteform. The file contains the title of the file, the input file name, the units (curies, Becquerels or grams), a list of all container locations by node number and spatial position. Then for each rdionuclide at each time step, the mass released from the container in that node.

Table 8.7 LEACHTMS File. Total mass release. 
IAEA COURSE / TEST CASE

Input file name srczone.inp

Mass in Curies

\section{WASTEFORM RELEASES FILE - PART TWO}

There are 20 wasteforms

\begin{tabular}{rrrr} 
& \multicolumn{3}{c}{ Total mass release a } \\
iso & Time & (yr) & Total \\
5 & 6 & 7 & 8 \\
9 & 10 & 11 & 12 \\
13 & 14 & 15 & 16 \\
17 & 18 & 19 & 20
\end{tabular}

TC-99 5.101E-02 1.080E+01 1.080E+00 1.080E+00 1.080E+00 $1.080 \mathrm{E}+00$

$\begin{array}{llllll}1.080 \mathrm{E}+00 & 1.080 \mathrm{E}+00 & 1.080 \mathrm{E}+00 & 1.080 \mathrm{E}+00 & 1.080 \mathrm{E}+00 & 1.080 \mathrm{E}+00\end{array}$

$\begin{array}{lllllll}0.000 \mathrm{E}+00 & 0.000 \mathrm{E}+00 & 0.000 \mathrm{E}+00 & 0.000 \mathrm{E}+00 & 0.000 \mathrm{E}+00 & 0.000 \mathrm{E}+00\end{array}$

$0.000 \mathrm{E}+00 \quad 0.000 \mathrm{E}+00 \quad 0.000 \mathrm{E}+00 \quad 0.000 \mathrm{E}+00$

4.006E-02 4.006E-02 4.006E-02 4.006E-02 4.006E-02 4.006E-02

$0.000 \mathrm{E}+00 \quad 0.000 \mathrm{E}+00 \quad 0.000 \mathrm{E}+00 \quad 0.000 \mathrm{E}+00 \quad 0.000 \mathrm{E}+00 \quad 0.000 \mathrm{E}+00$

$0.000 \mathrm{E}+00 \quad 0.000 \mathrm{E}+00 \quad 0.000 \mathrm{E}+00 \quad 0.000 \mathrm{E}+00$

NP-237 5.101E-02 8.012E-01 8.012E-02

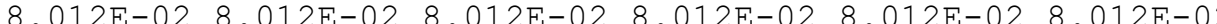

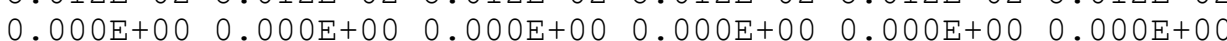

$0.000 \mathrm{E}+00 \quad 0.000 \mathrm{E}+00 \quad 0.000 \mathrm{E}+00 \quad 0.000 \mathrm{E}+00$

$\begin{array}{llllllll}\mathrm{TC}-99 & 1.046 \mathrm{E}-01 & 1.080 \mathrm{E}+01 & 1.080 \mathrm{E}+00 & 1.080 \mathrm{E}+00 & 1.080 \mathrm{E}+00 & 1.080 \mathrm{E}+00\end{array}$

$1.080 \mathrm{E}+001.080 \mathrm{E}+001.080 \mathrm{E}+001.080 \mathrm{E}+00 \quad 1.080 \mathrm{E}+001.080 \mathrm{E}+00$

$\begin{array}{lllllll}0.000 E+00 & 0.000 E+00 & 0.000 E+00 & 0.000 E+00 & 0.000 E+00 & 0.000 E+00\end{array}$

$0.000 \mathrm{E}+00 \quad 0.000 \mathrm{E}+00 \quad 0.000 \mathrm{E}+00 \quad 0.000 \mathrm{E}+00$

1.046E-01 4.009E-01 4.009E-02

4.009E-02 4.009E-02 4.009E-02 4.009E-02 4.009E-02 4.009E-02

$\begin{array}{lllllll}0.000 \mathrm{E}+00 & 0.000 \mathrm{E}+00 & 0.000 \mathrm{E}+00 & 0.000 \mathrm{E}+00 & 0.000 \mathrm{E}+00 & 0.000 \mathrm{E}+00\end{array}$

$0.000 \mathrm{E}+00 \quad 0.000 \mathrm{E}+00 \quad 0.000 \mathrm{E}+00 \quad 0.000 \mathrm{E}+00$

NP-237 1.046E-01 8.017E-01 8.017E-02

$0.000 \mathrm{E}+00 \quad 0.000 \mathrm{E}+00 \quad 0.000 \mathrm{E}+00 \quad 0.000 \mathrm{E}+00 \quad 0.000 \mathrm{E}+00 \quad 0.000 \mathrm{E}+00$

$0.000 \mathrm{E}+00 \quad 0.000 \mathrm{E}+00 \quad 0.000 \mathrm{E}+00 \quad 0.000 \mathrm{E}+00$

$1.081 \mathrm{E}+00 \quad 1.081 \mathrm{E}+00 \quad 1.081 \mathrm{E}+00 \quad 1.081 \mathrm{E}+00 \quad 1.081 \mathrm{E}+00 \quad 1.081 \mathrm{E}+00$

$\begin{array}{llllll}0.000 \mathrm{E}+00 & 0.000 \mathrm{E}+00 & 0.000 \mathrm{E}+00 & 0.000 \mathrm{E}+00 & 0.000 \mathrm{E}+00 & 0.000 \mathrm{E}+00\end{array}$

$0.000 \mathrm{E}+00 \quad 0.000 \mathrm{E}+00 \quad 0.000 \mathrm{E}+00 \quad 0.000 \mathrm{E}+00$

AM-241 1.610E-01 4.011E-01 4.011E-02 4.011E-02 4.011E-02

4.011E-02 4.011E-02 4.011E-02 4.011E-02 4.011E-02 4.011E-02

$\begin{array}{lllllll}0.000 E+00 & 0.000 E+00 & 0.000 E+00 & 0.000 E+00 & 0.000 E+00 & 0.000 E+00\end{array}$

$0.000 \mathrm{E}+00 \quad 0.000 \mathrm{E}+00 \quad 0.000 \mathrm{E}+00 \quad 0.000 \mathrm{E}+00$

$\begin{array}{llll}\mathrm{NP}-237 & 1.610 \mathrm{E}-01 & 8.022 \mathrm{E}-01 & 8.022 \mathrm{E}-02\end{array}$

8.022E-02 8.022E-02 8.022E-02 8.022E-02

8.022E-02 8.022E-02 8.022E-02

$0.000 \mathrm{E}+00 \quad 0.000 \mathrm{E}+00 \quad 0.000 \mathrm{E}+00 \quad 0.000 \mathrm{E}+00$ 


\subsection{Boundary Condition Files}

The boundary condition files (BOUND\&.DAT) is generated for each isotope. The file condtains the title of the input file, location(s) of trace nodes, number of data points in the file, and the units for the mass flow rate. This is followed by a table of time versus mass flow rate.

Table 8.8 BOUND1.DAT boundary condition file.

\begin{tabular}{|c|c|c|c|}
\hline \multicolumn{4}{|l|}{ Location of dat } \\
\hline Area of facili & Ey in unsaturated z & $=$ & $0.00 \mathrm{E}+00$ \\
\hline Number of data & points 220 & & \\
\hline Mass Flow rate & in units of Curies & per & second \\
\hline Time & Mass Flow Rate & & \\
\hline $0.00 E+00$ & $8.40 \mathrm{E}-20$ & & \\
\hline $5.10 \mathrm{E}-02$ & $8.40 E-20$ & & \\
\hline 1. $05 E-01$ & $5.51 E-17$ & & \\
\hline $1.61 E-01$ & $3.99 E-15$ & & \\
\hline 2. $20 E-01$ & $8.59 \mathrm{E}-14$ & & \\
\hline $2.82 \mathrm{E}-01$ & $8.52 \mathrm{E}-13$ & & \\
\hline $3.48 E-01$ & $4.97 E-12$ & & \\
\hline $4.17 E-01$ & $2.01 E-11$ & & \\
\hline 4.89E-01 & $6.15 \mathrm{E}-11$ & & \\
\hline $5.65 E-01$ & 1. $53 \mathrm{E}-10$ & & \\
\hline $6.45 E-01$ & $3.26 \mathrm{E}-10$ & & \\
\hline 7. $29 \mathrm{E}-01$ & $6.12 \mathrm{E}-10$ & & \\
\hline $8.17 \mathrm{E}-01$ & 1. $05 E-09$ & & \\
\hline $9.09 E-01$ & $1.65 E-09$ & & \\
\hline 1. $01 E+00$ & $2.45 E-09$ & & \\
\hline 1.11E+00 & $3.45 E-09$ & & \\
\hline 1. $22 \mathrm{E}+00$ & $4.66 E-09$ & & \\
\hline $1.33 E+00$ & $6.02 E-09$ & & \\
\hline $1.45 \mathrm{E}+00$ & $7.57 \mathrm{E}-09$ & & \\
\hline $1.57 \mathrm{E}+00$ & $9.28 E-09$ & & \\
\hline 1. $70 \mathrm{E}+00$ & $1.11 \mathrm{E}-08$ & & \\
\hline $1.84 \mathrm{E}+00$ & $1.30 E-08$ & & \\
\hline 1.99E+00 & 1. $50 \mathrm{E}-08$ & & \\
\hline $2.14 \mathrm{E}+00$ & $1.70 E-08$ & & \\
\hline $2.30 \mathrm{E}+00$ & $1.89 E-08$ & & \\
\hline $2.47 \mathrm{E}+00$ & 2. $09 \mathrm{E}-08$ & & \\
\hline $2.65 \mathrm{E}+00$ & $2.27 E-08$ & & \\
\hline $2.83 E+00$ & $2.45 E-08$ & & \\
\hline $3.03 E+00$ & $2.61 E-08$ & & \\
\hline $3.23 E+00$ & $2.76 \mathrm{E}-08$ & & \\
\hline $3.45 E+00$ & $2.88 E-08$ & & \\
\hline $3.68 \mathrm{E}+00$ & $2.99 E-08$ & & \\
\hline $3.91 \mathrm{E}+00$ & $3.08 E-08$ & & \\
\hline $4.16 \mathrm{E}+00$ & $3.15 E-08$ & & \\
\hline $4.43 E+00$ & $3.20 E-08$ & & \\
\hline $4.70 E+00$ & $3.23 E-08$ & & \\
\hline 5. $00 \mathrm{E}+00$ & $3.23 E-08$ & & \\
\hline $5.30 E+00$ & $3.20 E-08$ & & \\
\hline $5.62 \mathrm{E}+00$ & $3.16 \mathrm{E}-08$ & & \\
\hline
\end{tabular}




\section{INSTRUCTIONS FOR USE OF GRAFXT}

GRAFXT was created to provide the DUST user with a means of rapidly analyzing the primary output, (concentrations and fluxes), of the code. GRAFXT takes the files TRACECND.DAT, TRACEFXD.DAT, and CONCNT.DAT and displays them on a video display device. The created graph is a linear $\mathrm{X}-\mathrm{Y}$ plot of the data.

GRAFXT was written using LAHEY FORTRAN version 5 and should work with any monitor. However, the code was written in the early 1990's and works only with 16 bit architecture machines. A new version of GRAFXT was written to be compatible with newer computer architectures (e.g. 32 bit machines). This code, GRAFMS can also plot the output from DUST-MS. The major difference is that in DUST-MS output, there can be multiple species. The remainder of this chapter discusses the operation of GRAFXT . GRAFMS is similar, but has a better user interface. Although GRAFXT works on monochrome as well as color monitors, a color monitor is recommended as the display is greatly enhanced in this case.

GRAFXT is menu driven. Decisions on the variables to plot are resolved through interactive questioning of the user. As done in the DUSTIN chapter, text that appears on the console will appear in a double border box. Responses by the user will be italicized within this box.

\subsection{Selection of the File for Plotting}

The first series of questions involves determining which file should be used for plotting purposes.

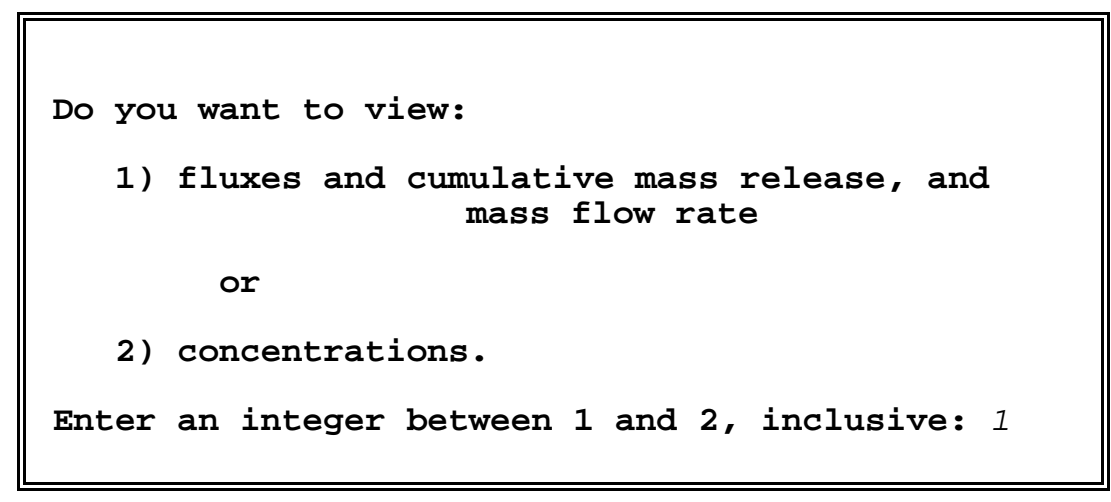

In this case, TRACEFXD.DAT was selected. If the user selected concentrations, the code would ask the question which concentration file (TRACECND.DAT or CONCNT.DAT) should be opened. 


\subsection{Selection of the Plot Variables}

After selecting the file, the type of plot must be determined. GRAFXT can take any one of the three files and plot the variables versus time or location. In general, the trace files, which have the variables at fixed locations at a large number of time steps, were designed to be plotted versus time at fixed locations. The CONCNT.DAT file was written to provide a plot of concentration versus location at fixed times. 
Do you want to plot your selection:

1) versus time at fixed locations or

2) versus location at fixed times.

Enter an integer between 1 and 2, inclusive: 1

\subsubsection{Number of plots per graph - Independent variable is time}

In this case, the user selected a plot with time as the independent variable. At this point, the data file is read, the title card which labels each plot is printed and the number of trace nodes on the file are printed. The user is asked to provide the nodes at which a plot is requested. More than one plot can be drawn on the graph at one time. For color monitors, it is recommended that the number of plots does not exceed the number of different colors available. For monochrome monitors, it is recommended that only a few plots per graph be made due to difficulty in differentiating between plots.

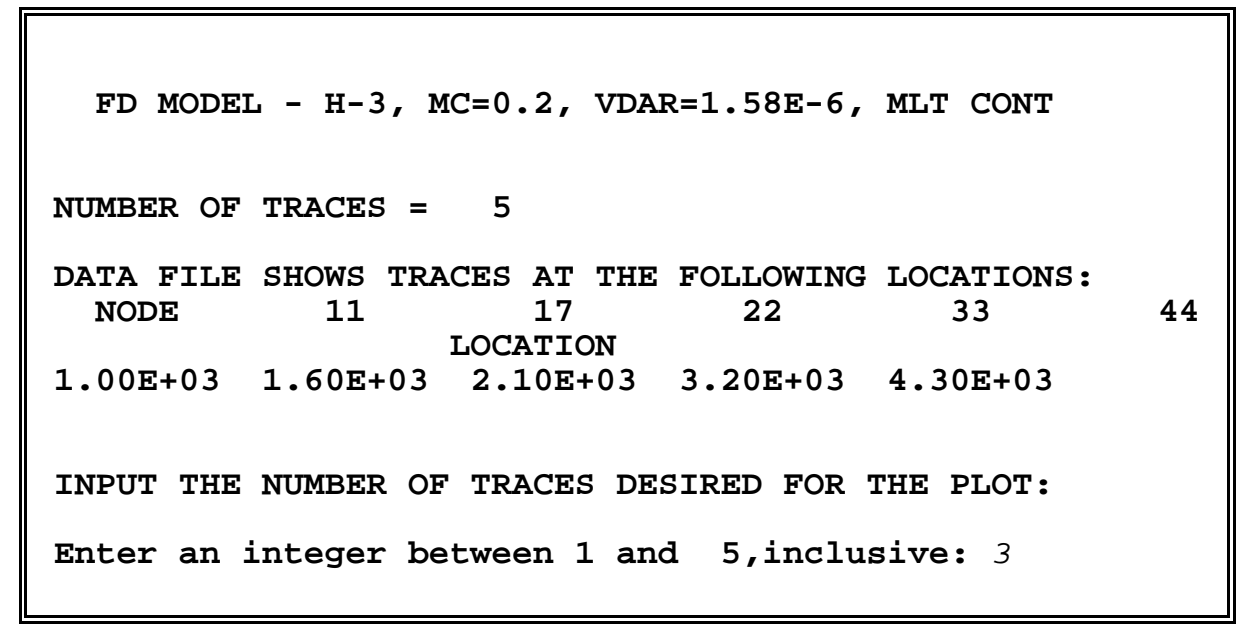

In the previous example, 3 plots are requested. The code then prompts the user for the node numbers to be used for plotting.

INPUT THE NODE NUMBERS FOR THE TRACES:

$\begin{array}{lll}17 & 22 & 33\end{array}$ 
The node numbers can be input on a single line, separated by a space as in the example. If the code user requested that all five nodes be plotted, the code would automatically define the five plot nodes and the previous question is skipped.

\subsubsection{Number of plots per graph - Independent variable is distance}

If the user requested distance as the independent variable, the GRAFXT code asks a different series of questions. Namely, it determines the fixed times at which a plot is requested. The code first prints the locations of all of the nodes in the file. Then the table of output times is printed.

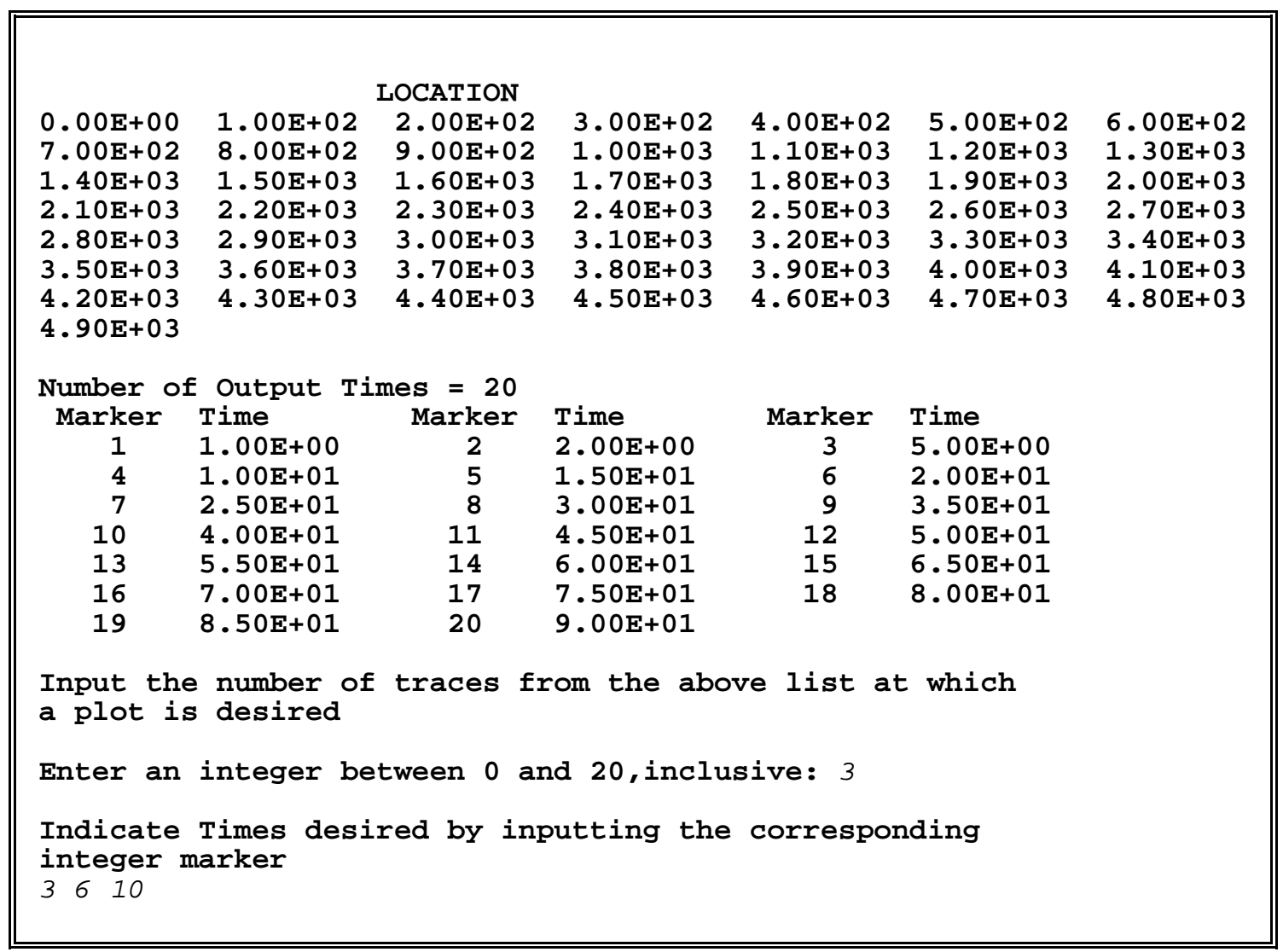

In this example, the file contained 20 output times. The times at which a plot are requested are determined through the integer markers. This was done to prevent problems with comparisons between real numbers as the code checks to make sure that the requested time is in the output file. Therefore, specifying 3, 6, and 10 as the plot markers gives output at times of 5, 20 and 40 years.

\subsection{Creating a Plot}


At this point, the code prompts the user if the data should be plotted as is, (automatic generation) or if the user wants to customize the plot.

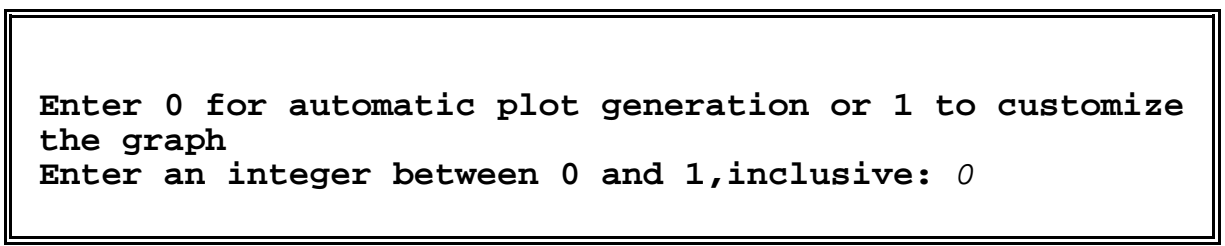

If automatic generation is used, the screen is changed to graphics mode and the plot is drawn.

Figure 9.1 displays a reproduction of a typical plot of flux versus time generated by GRAFXT. GRAFXT plots are on the video display and not easily reproduced on a hard copy. Therefore, a reproduction of the plot was created using the spreadsheet QUATTRO.PRO. In Fig. 9.1, the Y-Axis label, FLUX X $10^{\wedge}-15$, is at the top. This is done to prevent the need to write parallel to the axis using FORTRAN, which is quite cumbersome. The value for the exponent, -15 , in this label is calculated by the code and is problem dependent. Beneath the Y-axis label, is the problem title supplied from the input file. The $\mathrm{X}$-axis is written beneath the values marked on this axis. The generation and labeling of all axis is performed automatically by the code and depends on the maximum and minimum values present. If the flux has both positive and negative values at different locations, this is handled automatically by the code. Although, different traces are highlighted with different markers in this figure. The actual output of GRAFXT does not include markers for data points. Curves are differentiated using different colors on the plot. Another difference between Figure 9.1 and a GRAFXT plot is the labels for the nodes appears on the left when GRAFXT is used.

After plotting, hitting any key will clear the screen and return to text mode.

\subsection{Customizing a Plot}

A plot can be customized in three ways, a) moving the location of the plot on the screen, b) redefining the axis labels, and c) limiting the range of the data. If customization is requested the following questions appear. 


\section{Species B Release}

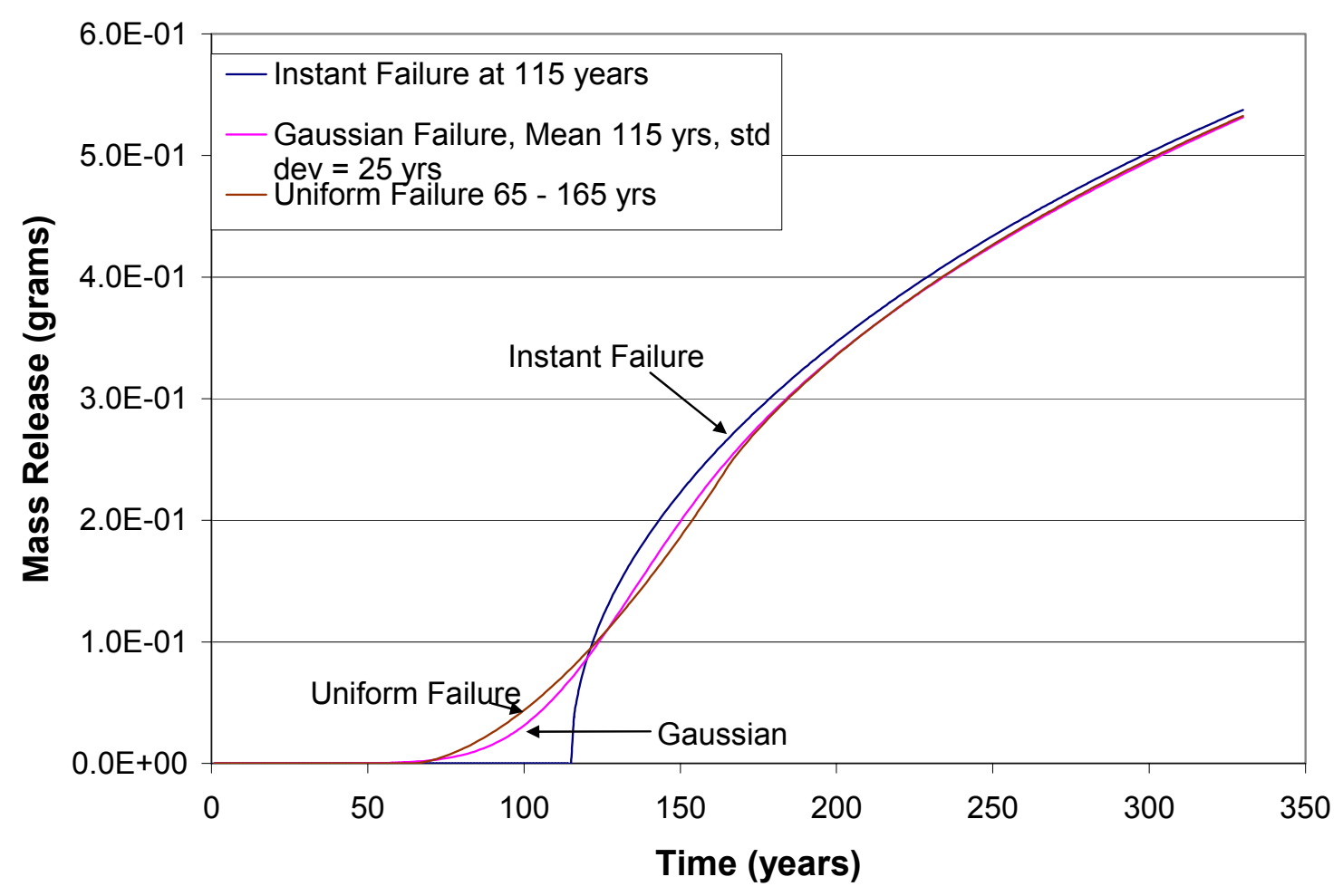

Figure 9.1 Typical graph created by the program GRAFMS. 


\subsubsection{Changing the Plot Origin}

The code GRAFXT selects the screen origin of the plot automatically. The value is chosen to get the maximum resolution on the screen. Under unusual circumstances, this may cause the axis labels to be off the screen. In this case, the origin can be redefined. The code asks the following question:

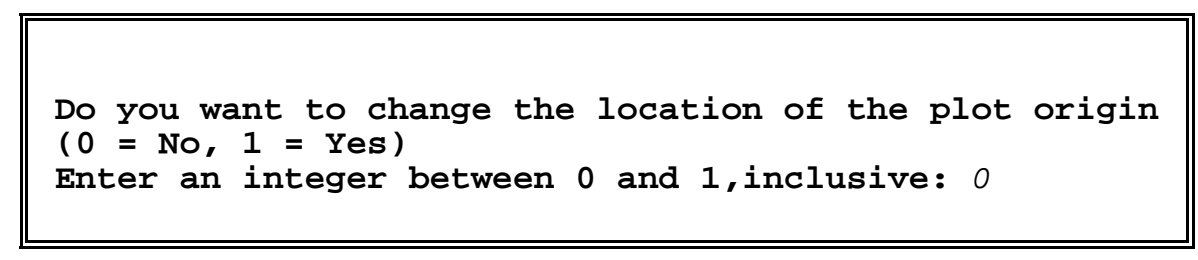

If the answer is yes, the code asks for the number of inches to move the $\mathrm{X}$ and $\mathrm{Y}$ coordinates of the origin.

\subsubsection{Changing the Axis Labels}

The code provides default labels for both the $\mathrm{X}$ and $\mathrm{Y}$ axis. If these are inadequate, they can be changed by responding 1 to the following question:

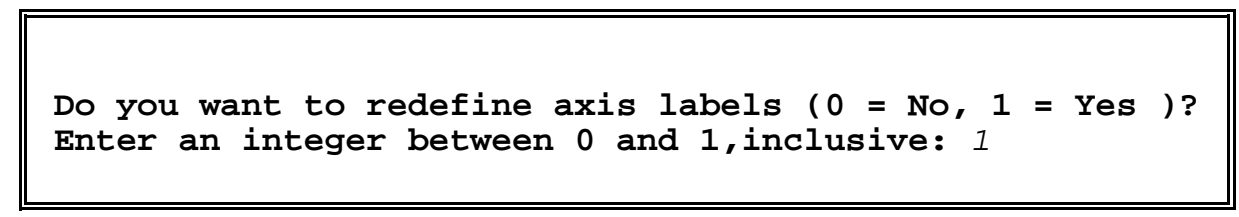

In this case, the code prompts the user for the both the $\mathrm{X}$ and $\mathrm{Y}$ axis labels. These are restricted to 20 characters in length.

\subsubsection{Limiting the range of the data}

GRAFXT provides a linear X-Y plot of the data. When automatic generation is used, all of the data is plotted on the graph. This causes poor resolution away from peak values. GRAFXT provides the user with a means of increasing the resolution at any point in the graph through specifying maximum and minimum values for both the $\mathrm{X}$ and $\mathrm{Y}$ parameters. The procedure follows: 


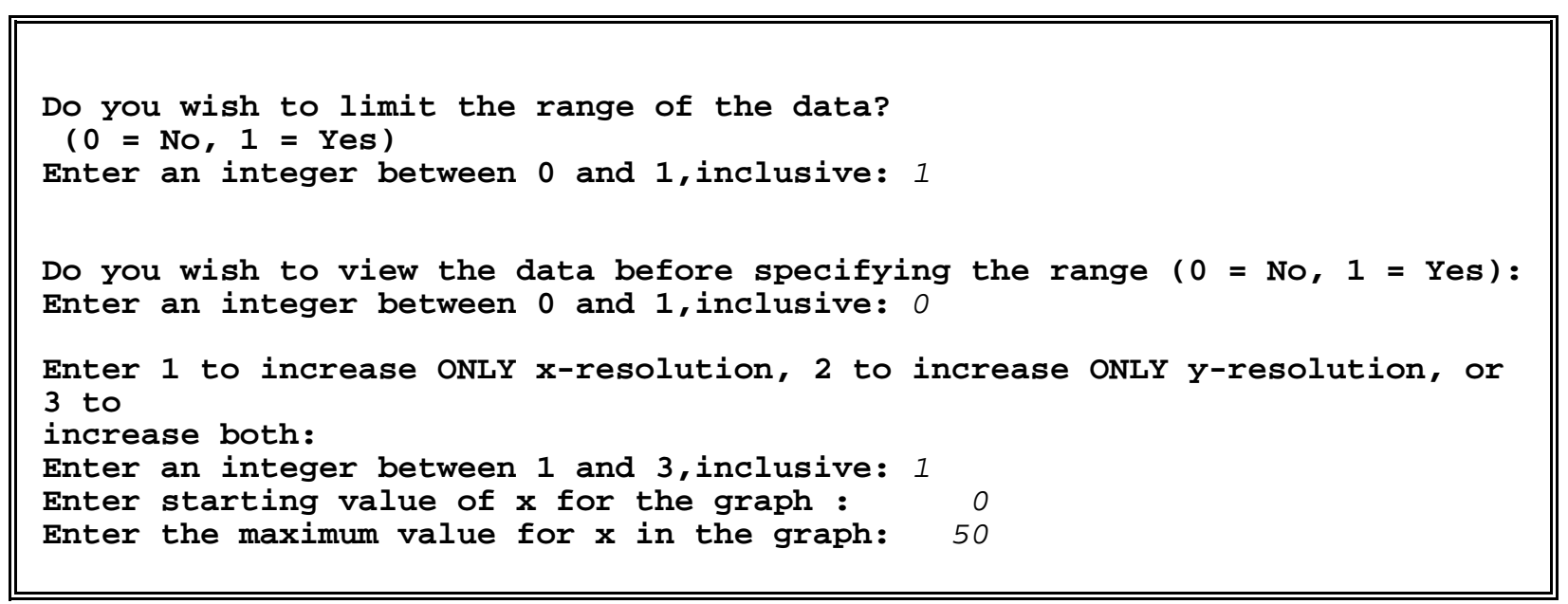

After a decision has been made to limit the data, the user is given the opportunity to view the selected plot data. If a value of 1 is input, all of the data at each of the plot locations is printed. This is useful when trying to determine appropriate ranges for the data. After this section, the user is provided three options for limiting the range of the data as presented above. In the example, the user excludes all points in which the value of $\mathrm{X}$ is outside the range of $0-50$. At this point, the user is asked to view the data before plotting. If this option is selected, only data that falls within the specified range is printed. If the range is inappropriate, the user can return and specify a new range. After the user is satisfied with the data, a plot is generated.

Use of this option is particularly useful in two cases: a) often in a simulation, releases are peaked around a small change in time. If this happens, the minimum and maximum values for time can be changed to provide better resolution about the peak. And, b) In certain cases, the values away from the peak are required. Due to the linear plot, these appear as near zero. By limiting the range of times to be far away from the peak, higher resolution can be obtained in these areas.

\subsection{Creating Additional Plots}

Although GRAFXT can creates one plot at any given time, after a plot has been viewed, the following question appears:

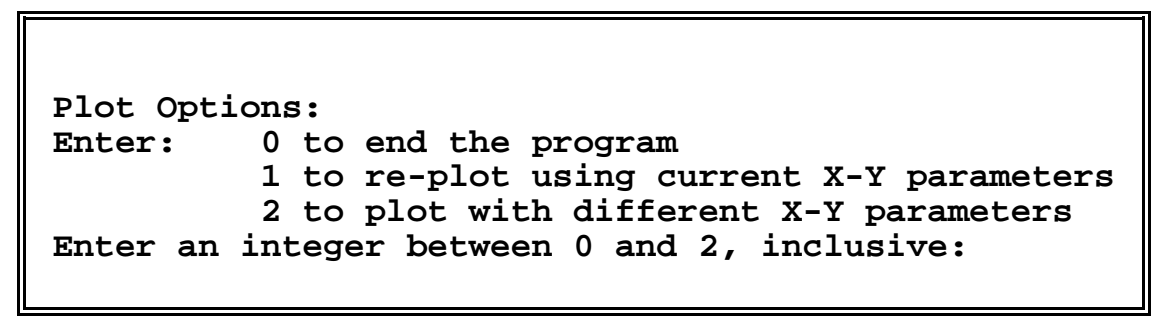


Option 0 ends the program. Option 1 permits further plots to be made with the current plot file. For example, if the previous plot was the flux versus time at specified locations, the user could request another plot with a restricted range of data, or create a similar plot with different trace locations. For the flux file, TRACEFXD, the user could also use the same locations and plot the cumulative mass release or the mass flow rate. With this option, the user can not change from plots of the function versus time to plots versus location, or vice versa. Option 2 returns the user to the initial menu and is equivalent to exiting the code and beginning over. All plot options are available. 


\section{CONCLUSIONS}

The DUST-MS code, which predicts the release and transport of radioactive contaminants from a LLW disposal facility, has been developed, implemented, and tested. The DUST-MS code contains models to predict the important processes (fluid flow, container degradation, wasteform leaching, and radionuclide transport) related to release and transport of radionuclides within the disposal facility.

The DUST-MS code improves upon existing codes in that more flexibility is allowed in order to model the various waste stream/wasteform/container systems while still retaining relatively simple models that do not require extensive computer time or provide an undue burden on the code user in terms of input requirements.

To facilitate creation of an input deck, the code DUSTWIN has been written. It is a menu driven program that guides the user through all of the steps required to create an input deck. Upon completion of these steps, DUSTWIN will write an input file for use by DUST-MS.

In addition, to permit rapid analysis of the output data created by DUST-MS, a graphics program GRAFMS has been written. GRAFMS is capable of plotting the concentration, flux, or mass that has passed a fixed location as a function of either time or location directly on a video console.

The DUST-MS code improves upon the codes that currently exist, yet still retains a simplified structure in their treatment of the complex phenomena involved in the release and transport of radionuclides within a disposal facility. As such, the models are prone to misuse through improper choice of the input parameters. Enough emphasis cannot be placed on the need for justification and documentation of the choices for the input parameters. The model predictions are only as good as the least justifiable input.

In using DUST-MS, as with all computer models, the validity of the predictions relies heavily on the validity of the input parameters. Often, the largest uncertainties arise from uncertainty in the input parameters. Therefore, it is crucial to document and support the use of these parameters. The DUST code, because of its ability to compute release rates quickly, will be extremely useful for screening to determine the radionuclides released at the highest rate, parameter sensitivity analyses, and, with proper choice of the input parameters, provide upper bounds to release rates. 


\section{REFERENCES}

[ANS, 1986]

American Nuclear Society, "Measurement of the Leachability of Solidified Low-Level Radioactive Wastes," Prepared by the American Nuclear Society Standards Committee Working Group ANS-16.1, 1986.

[Baes, 1983]

C.F. Baes, III, and R.D. Sharp, "A Proposal for Estimation of Soil Leaching and Leaching Constants for Use in Assessment Models," Journal of Environmental Quality, Vol. 12, No. 1, pp. 17-24, 1983.

[Chu, 1991]

M.S.Y. Chu, M.W. Kozak, J.E. Campbell, and B.M. Thompson, "A Self-Teaching Curriculum for the NRC/SNL Low-Level Waste Performance Assessment Methodology," NUREG/CR-5539, SAND900585, Sandia National Laboratories, 1991.

[Clifton, 1989]

J.R. Clifton and L.I. Knab, "Service Life of Concrete," NUREG/CR-5466, NISTIR89-4086, National Institute of Standards and Technology, 1989.

[Cowgill, 1992]

M.G. Cowgill and T.M. Sullivan, "Source Term Evaluation for Low-Level Radioactive Waste Disposal Performance," NUREG/CR-5911, BNL-NUREG-52334, Brookhaven National Laboratory, 1993.

[Cowgill, 1992a]

M.G. Cowgill and T.M. Sullivan, "Source Term Evaluation for Performance Assessment of LLW Disposal," WM '92, ed. R.G. Post, Tucson, AZ, 1992.

[DOE, 1987]

Conference of Radiation Control Program Directors, Inc., "Compilation of State-by-State Low-Level Radioactive Waste Information," DOE/ID/12377-T1, October, 1987.

[DOE, 1990]

U.S. Department of Energy, "Integrated Data Base for 1990: U.S. Spent Fuel and Radioactive Waste Inventories, Projections, and Characteristics," DOE/RW-0006, Rev. 6, Oak Ridge National Laboratory, October, 1990.

[FR, 1982]

Federal Register, "Licensing Requirements for Land Disposal of Radioactive Waste," Vol. 47, No.248, Monday, December 27, 1982. 
[Gee, 1988]

G.W. Gee and D. Hillel, "Groundwater Recharge in Arid Regions: Review and Critique of Estimation Methods," Hydrological Processes, Vol. 2, p. 255-266, 1988.

[Gerhold, 1981]

W.F. Gerhold, E. Escalante, and B.T. Sanderson, "The Corrosion Behavior of Selected Stainless Steels in Soil Environments," NBSIR 81-2228, National Bureau of Standards, 1981.

[Gruhlke, 1986]

J.M. Gruhlke, J. Neiheisel, and L. Battist, "Estimates of the Quantities, Form and Transport of Carbon14 in Low-Level Radioactive Waste," EPA 520/1-86-019, U.S. Environmental Protection Agency, 1986.

[Higginbotham, 1983]

L.R. Higginbotham, "Final Waste Classification and Waste Form Technical Position Papers," United States Nuclear Regulatory Commission, May 11, 1983.

[Huyakorn, 1989]

P.S. Huyakorn, J.B. Kool, and J.B. Robertson, "VAM2D - Variably Saturated Analysis Model in Two Dimensions," NUREG/CR-5352, HGL/89-01, HydroGeoLogic, Inc., 1989.

[Kozak, 1989]

M.W. Kozak, et. al., "Background Information for the Development of a Low-Level Waste Performance Assessment Methodology: Selection and Integration of Models," NUREG/CR-5453, SAND89-2509, Volume 3, Sandia National Laboratories, 1989.

[Kozak, 1989a]

M.W. Kozak, et. al., "Background Information for the Development of a Low-Level Waste Performance Assessment Methodology: Identification and Recommendation of Computer Codes," NUREG/CR-5453, SAND89-2509, Volume 4, Sandia National Laboratories, 1989.

[Kozak, 1990]

M.W. Kozak, et. al., Background Information for the Development of a Low-Level Waste Performance Assessment Methodology: Computer Code Implementation and Assessment," NUREG/CR-5453, SAND89-2509, Volume 5, Sandia National Laboratories, 1990.

[Kunz, 1982]

C.O. Kunz, "Radioactive Gas Production and Venting at a Low-Level Radioactive Waste Burial Site," Nucl. Chem. Waste Management, Vol. 3, p. 185, 1982.

[Lohaus, 1991]

P.H. Lohaus, "Technical Position on Waste Form, Revision 1," U.S. Nuclear Regulatory Commission, January 24, 1991.

[Looney, 1987] 
B.B. Looney, M.W. Grant, and C.M. King, "Estimation of Geochemical Parameters for Assessing Subsurface Transport at the Savannah River Plant," DPST-85-904, DE87-013051, E.I. duPont de Nemours \& Co., 1987.

[Matuszek, 1983]

J.H. Matuszek and L.W. Robinson, "Respiration of Gases from Near-Surface Radioactive Waste Burial Trenches," Waste Management '83, ed. R.G. Post, pp. 423 - 427, Tucson, AZ, 1983.

[Mughabghab, 1989]

S.F. Mughabghab and T.M. Sullivan, "Evaluation of the Pitting Corrosion of Carbon Steels and Other Ferrous Metals in Soil Systems," Waste Management, Vol. 9, pp. 239-251, 1989.

[Otzunali, 1981]

O.I. Otzunali, G.C. Re', P.M. Moskowitz, E.D. Picazo, and C.J. Pitt, "Data Base for Radioactive Waste Management: Impact Analyses Methodology Report,"NUREG/CR-1759, Vol. 3, Dames and Moore, 1981.

[Otzunali, 1986]

O.I. Oztunali and G.W. Roles, "Update of Part 61 Impacts Analysis Methodology," NUREG/CR-4370, Vol. 1,1986.

[Roache, 1976]

P.J. Roache, Computational Fluid Dynamics, Hermosa Publishers, Albuquerque, NM, 1976.

[Roles, 1990]

G.W. Roles, "Characteristics of Low-Level Radioactive Waste Disposed During 1987 Through 1989", NUREG-1418, U.S. Nuclear Regulatory Commission, 1990.

[Romanoff, 1957]

M. Romanoff, "Underground Corrosion," National Bureau of Standards Circular 579, 1957.

[Shuman, 1991]

R. Shuman, N. Chau, and V. Rogers, "Improved Modeling of Engineered Barriers for Low-Level Waste Disposal," presented at Waste Management '91, Tucson, AZ, 1991.

[Soo, 1990]

P. Soo and L.W. Milian, "The Impact of LWR Decontaminations on Solidification, Waste Disposal and Associated Occupational Exposure," NUREG/CR-3444, BNL-NUREG-51699, Vol. 7, Brookhaven National Laboratory, 1990. 
[Starmer, 1988]

R.J. Starmer, L.R. Deering, and M.F. Weber, "Performance Assessment Strategy for Low-Level Waste Disposal Sites," in the proceedings of the Tenth Annual DOE Low-Level Waste Management Conference, Denver, 1988.

[Streigel, 1985]

R.G. Streigel, "Methods for Determining the Transport of Radioactive Gases in the Unsaturated Zone," Department of Energy Low-Level Waste Management Participants Meeting, p. 579 - 587, Denver, CO., 1985.

[Sullivan, 1988]

T.M. Sullivan, C.R. Kempf, C.J. Suen, and S.M. Mughabghab, "Low-Level Radioactive Waste Source Term Model Development and Testing," NUREG/CR-5204, BNL-NUREG-52160, Brookhaven National Laboratory, 1988.

[Sullivan, 1988a]

T.M. Sullivan, C.J. Suen, and E. Sweeney, "Low-Level Waste Source Term Evaluation Quarterly Progress Report: July - September, 1988," WM-3276-7, Brookhaven National Laboratory, 1988.

[Sullivan, 1989]

T.M. Sullivan and C.J. Suen, "Low-Level Waste Shallow Land Disposal Source Term Model: Data Input Guides," NUREG/CR-5387, BNL-NUREG-52206, Brookhaven National Laboratory, 1989.

[Sullivan, 1991]

T.M. Sullivan and C.J. Suen, "Low-Level Waste Source Term Model Development and Testing," NUREG/CR-5681, BNL-NUREG-52280, Brookhaven National Laboratory, 1991.

[Sullivan, 1991a]

T.M. Sullivan, "Selection of Models to Calculate the LLW Source Term," NUREG/CR-5773, BNLNUREG-52295, Brookhaven National Laboratory, 1991.

[Sullivan, 1991b]

T.M. Sullivan and M.G. Cowgill, "Evaluation of the Data Available for Estimating Release Rates from Commercial Low-Level Waste Packages," BNL-NUREG-4676, DOE Low-Level Waste Management Conference, November 1991, Atlanta, GA.

[Sullivan, 1992]

T.M. Sullivan, "Development of DUST: A Computer Code that Calculates Release Rates from a LLW Disposal Unit," Waste Management '92, ed. R.G. Post, Tucson, AZ, 1992. 
[Thibault, 1990]

D.H. Thibault, M.I. Sheppard, and P.A. Smith, "A Critical Compilation and Review of Default Soil/Solid Liquid Partition Coefficients, $\mathrm{K}_{\mathrm{d}}$, for Use in Environmental Assessments," AECL-10125, Atomic Energy of Canada Limited, 1990.

[Travis, 1991]

B.T. Travis and K.H. Birdsell, "TRACR3D: A Model of Flow and Transport in Porous Media: Model Description and User's Manual," LA-11798-M, Los Alamos National Laboratory, 1991.

[Van Genuchten, 1978]

M. Th. Van Genuchten, "Mass Transport in Saturated-Unsaturated Media: One-Dimensional Solution," Research Report 78-WR-11, Water Resources Program, Department of Civil Engineering, Princeton University, 1978.

[Walton, 1990]

J.C. Walton, L.E. Plansky, and R.W. Smith, "Models for Estimation of Service Life of Concrete Barriers in Low-Level Radioactive Waste Disposal," NUREG/CR-5542, EGG-2597, EG\&G Idaho National Engineering Laboratory, 1990.

[Yeh, 1987]

G.T. Yeh, "FEMWATER: A Finite Element Model of Water Flow Through Saturated-Unsaturated Porous Media - First Revision," ORNL-5567/R1, Oak Ridge National Laboratory, 1987. 


\section{APPENDIX A: MATHEMATICAL DESCRIPTION OF THE}

\section{MODELS SELECTED FOR SOURCE TERM ANALYSIS}

The mathematical details pertaining to the models selected for source term analysis are described in this Appendix. The models that have involved mathematical descriptions cover the processes of wasteform diffusion release, and radionuclide transport. The complete models for water flow, container degradation, rinse release, and uniform release are presented in Chapter 2.

There are no models, per se, for the disposal facility radionuclide inventory. Rather, the inventory will be treated as a known quantity. The methodology required to obtain the inventory is also discussed in Chapters 2 and 3.

\section{Wasteform Release}

\section{$\underline{\text { Diffusion-Controlled Release }}$}

Conceptually, release from many solidified wasteforms can be described as a diffusioncontrolled process. Two models, differing only in geometry, are provided. In these models, it is assumed that, at the outer edge of the wasteform, the radionuclide concentration is zero. This assumes that transport processes away from the wasteform are fast enough to remove any radionuclides supplied by diffusion out of the wasteform. Although this situation will never be realized exactly, it does provide for the maximum diffusive release rate from the wasteform. Further, in many cases of practical interest, the solution concentration outside of the wasteform will not increase to a level such that it will markedly influence release.

In both models, we analytically solve the diffusion equation corrected for decay.

$$
\frac{\partial C}{\partial t}=\Delta \cdot D \Delta C-\lambda C
$$

where $\mathrm{D}=$ the effective diffusion coefficient, $\mathrm{C}$ is the concentration, and $\lambda$ is the radioactive decay constant. 
The initial condition assumes a uniform concentration throughout the wasteform:

$$
C(x, y, z, 0)=C_{o}
$$

The boundary conditions assume symmetry about the midplane of the wasteform and zero concentration at the outer edge.

$$
\begin{aligned}
& C\left(x_{b}, y, z, t\right)=0 \\
& C\left(x, y_{b}, z, t\right)=0 \\
& C\left(x, y, z_{b}, t\right)=0
\end{aligned}
$$

where the subscript b denotes a boundary.

Solution of Eqn. (A.1) subject to the initial and boundary conditions gives the concentration at any location within the wasteform. However, the quantity of interest is the release rate, which is the mass flux integrated over the surface area.

$$
Q(t)=\int d S \bullet J_{s}
$$

where:

$\mathrm{Q}(\mathrm{t})$ is the mass release per unit time; and $\mathrm{J}_{\mathrm{s}}$ is the mass flux at the surface.

For one-dimensional diffusion-controlled release,

$$
J_{s}=-D \frac{\partial C\left(x_{s}\right)}{\partial x}
$$

where $\mathrm{x}_{\mathrm{S}}$ denotes a surface of the wasteform. 


\section{Cylindrical Geometry}

The expression for the mass release rate from a cylindrical wasteform of height, $2 \mathrm{~L}$, and radius $\mathrm{R}$ is [Pescatore, 1991]:

$$
Q(t)=Q_{r}(t)+Q_{z}(t)
$$

where:

$$
Q_{r}(t)=Q_{r}(\lambda ; t)=\frac{32}{\pi^{2} R^{2}} \bullet D C_{o} V e^{-\lambda t} S_{p}(t) S_{q}(t)
$$

And,

$$
Q_{z}(t)=Q_{z}(\lambda ; t)=\frac{8}{L^{2}} \bullet D C_{o} V e^{-\lambda t} S_{c}(t) S_{d}(t)
$$

Here,

$$
\begin{gathered}
S_{c}(t)=\sum_{m=1}^{\infty} \frac{e^{-(\beta m / R)^{2} D t}}{\left(\beta_{m}\right)^{2}} \\
S_{p}(t)=\sum_{n=1}^{\infty} \frac{e^{-[(2 n-1) \pi / 2 L]^{2} D t}}{(2 n-1)^{2}} \\
S_{q}(t)=\sum_{m=1}^{\infty} e^{-(\beta m / R)^{2} D t}
\end{gathered}
$$




$$
S_{d}(t)=\sum_{n=1}^{\infty} e^{-[(2 n-1) \pi / 2 L]^{2} D t}
$$

where the $\beta_{\mathrm{m}}$ are the zeroes of the zero-th order cylindrical Bessel function and are presented in Table A.1.

Table A.1 Values of the parameters $\beta_{\mathrm{m}}$ for $\mathrm{m}=1$ to 20 .

These parameters satisfy the equation $\mathrm{J}_{0}\left(\beta_{\mathrm{m}}\right)=0$, with $\mathrm{J}_{0}(\mathrm{x})$ the zeroth order cylindrical Bessel function.

\begin{tabular}{cccc}
$\mathrm{m}$ & $\beta_{\mathrm{m}}$ & $\mathrm{m}$ & $\beta_{\mathrm{m}}$ \\
\cline { 2 - 4 } 1 & 2.4048255577 & 11 & 33.7758202136 \\
2 & 5.5200781103 & 12 & 36.9170983537 \\
3 & 8.6537279129 & 13 & 40.0584257646 \\
4 & 11.7915344391 & 14 & 43.1997917132 \\
5 & 14.9309177086 & 15 & 46.3411883717 \\
6 & 18.0710639679 & 16 & 49.4826098974 \\
7 & 21.2116366299 & 17 & 52.6240518411 \\
8 & 24.3524715308 & 18 & 55.7655107550 \\
9 & 27.4934791320 & 19 & 58.9069839261 \\
10 & 30.6346064684 & 20 & 62.0484691902 \\
& & &
\end{tabular}

Efficient means of calculating the infinite series in Eqns. (A.9 - A.12) have been developed [Pescatore, 1991] and are implemented in the source term code.

\section{$\underline{\text { Rectangular Geometry }}$}

Given a rectangular-shaped, radioactive wasteform with dimensions $2 \mathrm{a}, 2 \mathrm{~b}$, and $2 \mathrm{c}$ along the $\mathrm{x}, \mathrm{y}$, and $\mathrm{z}$ directions, respectively, and given the same assumptions about the wasteform as were utilized earlier for the cylindrical case, the concentration of radioactive species in the rectangular block is as follows:

$$
C(x, y, z, t)=\frac{64}{\pi^{3}} C_{o} e^{-\lambda t} T_{x}(a, t) T_{y}(b, t) T_{z}(c, t)
$$


where the generic function $\mathrm{T}_{\mathrm{u}}(\mathrm{L}, \mathrm{t})$ represents the open series:

$$
T_{u}(L, t)=\sum_{n=1}^{\infty}(-1)^{n+1} \bullet \frac{e^{-[(2 n-1) \pi / 2 L]^{2} D t}}{2 n-1} \bullet \cos \left(\frac{2 n-1}{2 L} \pi u\right)
$$

Equation (A.13) is obtained from the mathematically analogous expression for the temperature distribution within a heat-conducting parallelepiped.

Following [Pescatore, 1991], the release rate per unit area across the face $\mathrm{x}=\mathrm{a}$ of the wasteform is then:

$$
-\left.D \frac{\partial C}{\partial x}\right|_{x=a}=\frac{64}{\pi^{3}} C_{o} D e^{-\lambda t} T_{y}(b, t) T_{z}(c, t)\left[\frac{\pi}{2 a} S_{d}(a, t)\right]
$$

where the function $S_{d}(a, t)$ is the open series [A.12] with $L=a$. The total release rate, $Q_{a}$, across the face $\mathrm{x}=\mathrm{a}$ is obtained upon integration of Eqn. [A.15] over the entire area of this face, yielding:

$$
Q_{x=a}(\lambda ; t)=\frac{512}{\pi^{4}} C_{o} D \frac{b c}{a} e^{-\lambda t} S_{p}(b, t) S_{p}(c, t) S_{d}(a, t)
$$

where the functions $S_{p}(b, t)$ and $S_{p}(c, t)$ represent the open series (A.10) evaluated with $L=b$ and $L=c$, respectively. Analogous expressions for the total release rates from the other surfaces of the wasteforms can be obtained by substituting the dimensions of those surfaces in Eqn. (A.16).

\section{Radionuclide Transport}

Migration of contaminants through the disposal facility will be modeled in one of two ways: the multi-cell mixing cascade model or the finite difference solution of the advection-dispersion equation.

The mixing cell cascade approach is a generalization of the work performed by Sandia National Laboratories [Kozak, 1990] and relies on an analytical solution of the appropriate equations. With the analytical solution, the release rates from the disposal facility at any time are relatively easy to estimate. However, in order to obtain these solutions some restrictions on the generality of the problem must be made.

Both models begin with a mass balance for the contaminant within a control volume: 


$$
\begin{gathered}
\frac{\partial}{\partial t}(\theta C)=-\frac{\partial J}{\partial x} \\
-\lambda(\theta C+\rho S)-\frac{\partial}{\partial t}(\rho S) \\
+q
\end{gathered}
$$

where:

$\mathrm{C}=$ solution concentration;

$\theta=$ the volumetric moisture content of the region (dimensionless);

$\lambda=$ radioactive decay constant;

$\mathrm{S}=$ adsorbed concentration, the mass adsorbed per unit mass of the solid;

$\rho=$ bulk density of the solid; and

$\mathrm{q}=$ source/sink term used to model release from the waste form.

In Eqn. (A.17) we assume that the mass adsorbed on the solid surfaces is in equilibrium with the mass in solution. Further, we assume that this equilibrium can be described using a concentration independent partition coefficient, $\mathrm{K}_{\mathrm{d}}$, as follows:

$$
S=K_{d} C
$$

Using the above relationship for $\mathrm{S}$, assuming that the bulk density remains constant within the disposal facility and rearranging Eqn. (A.17), the following equation is obtained:

$$
\begin{array}{r}
\frac{\partial}{\partial t}(R \theta C)=-\frac{\partial J}{\partial x} \\
-\lambda \theta R C+q
\end{array}
$$

where:

$$
R=1+\frac{\rho K_{d}}{\theta}
$$


$\mathrm{R}$ is known as the retardation coefficient.

The rate of change in mass flux with position provides a measure of the flow rate of mass entering or leaving the control volume and contains terms related to movement through the processes of diffusion/dispersion and advection. The mass flux is:

$$
\begin{array}{r}
J=-\theta D \frac{\partial C}{\partial x}+V_{d} C \\
D=D_{m}+\frac{a_{t}\left|V_{d}\right|}{\theta} ;
\end{array}
$$

where: $\mathrm{D}=$ the diffusion-dispersion coefficient, $\mathrm{D}_{\mathrm{m}}=$ effective diffusion coefficient;

$\mathrm{a}_{\mathrm{t}}=$ transverse dispersivity;

$\mathrm{V}_{\mathrm{d}}=$ Darcy velocity; yields:

Using Eqn. (A.21) in the mass balance equation with a linear sorption isotherm, Eqn. (A.19),

$$
\begin{aligned}
\frac{\partial}{\partial t}(R \theta C) & =\frac{\partial}{\partial x}\left(\theta D \frac{\partial C}{\partial x}\right)-\frac{\partial}{\partial x}\left(V_{D} C\right) \\
& -\lambda \theta R C+q
\end{aligned}
$$

\section{Mixing-Cell Cascade}

The mixing cell cascade model divides the disposal facility into a number of uniform size mixing cells as depicted in Figure A.1. Within each cell it is assumed that the contaminant released from the waste form is uniformly mixed thereby giving a uniform solution concentration. In order to obtain an analytical solution the following assumptions are made:

a) Migration is dominated by advective flow and therefore diffusion and dispersion can be ignored. 
b) The advection velocity, moisture content, and the retardation coefficient are constant throughout the disposal facility. Although these parameters will show variations due to different materials in the facility, they should be a selected to provide a representative average for the entire facility.

Using these assumptions in Eqn. (A.22) the transport equation for the $\mathrm{i}^{\text {th }}$ mixing cell becomes:

$$
\frac{\partial C_{i}}{\partial t}=-\frac{V_{D}}{\theta R} \frac{\partial C_{i}}{\partial x}-\lambda C_{i}+\frac{q_{i}}{\theta R}
$$

For a disposal facility of height $\mathrm{H}$, there are $\mathrm{N}$ mixing cells of height $\mathrm{h}(\mathrm{h}=\mathrm{H} / \mathrm{N})$. Using this definition of the size of the mixing cell and upwind differencing (because migration is controlled by advection) to estimate the spatial derivative, Eqn. (A.23) becomes:

$$
\frac{d C_{i}}{d t}=-\alpha N\left(C_{i}-C_{i-1}\right)-\lambda C_{i}+\beta N Q_{i}
$$

where:

$\mathrm{Q}=$ the total release rate from the wasteform;

$\alpha=\mathrm{V}_{\mathrm{d}} /(\theta \mathrm{RH})$;

$\beta=1 /\left(\theta \mathrm{RHA}_{\mathrm{f}}\right) ;$ and

$\mathrm{A}_{\mathrm{f}}=$ the area of the facility.

In this description, $\mathrm{HA}_{\mathrm{f}} / \mathrm{N}$ is the volume of a single mixing cell. 


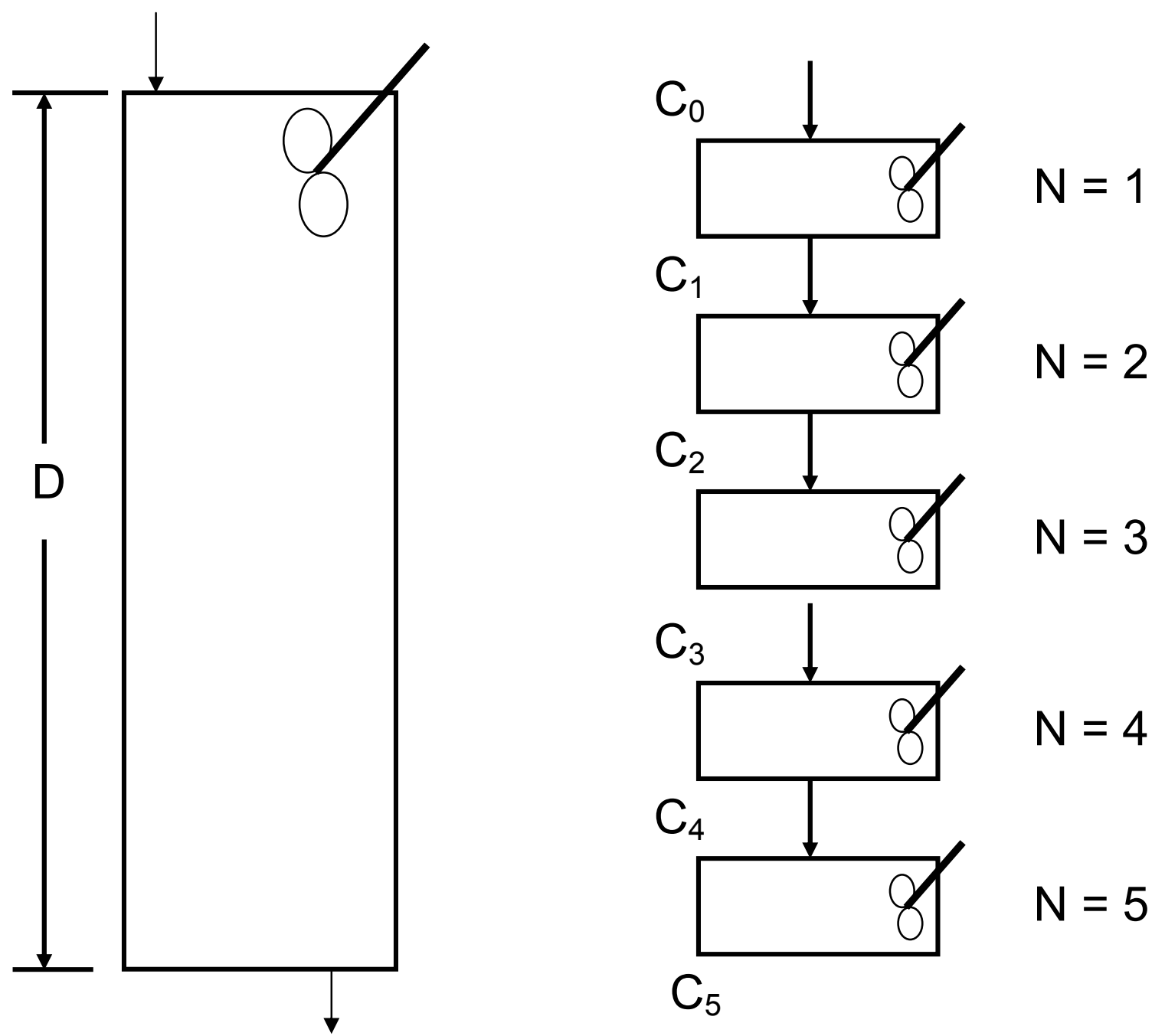

Figure A- 1 Schematic representation of the mixing cell cascade approach. (a) a single mixing cell, (b) multiple mixing cells. [From Kozak, 1990]

Equation (A.24) applies to each mixing cell. For the first cell, $\mathrm{C}_{\mathrm{i}-1}$ is set to zero. This is equivalent to assuming that no contaminant enters through the top of the facility. Therefore, we have a system of $\mathrm{N}$ coupled linear differential equations. This system of equations has been solved to provide an analytical solution for arbitrary wasteform sources, $\mathrm{q}_{\mathrm{i}}$, within each mixing cell.

The resulting expression is:

$$
C_{N}(t)=e^{-\lambda t} e^{-\alpha N t} \sum_{n=0}^{N-1} \frac{(\alpha N t)^{n}}{n !} C_{N-n}(0)
$$




$$
+\beta N e^{-\lambda t} e^{-\alpha N t} \sum_{n=0}^{N-1}(\alpha N)^{n} I_{n+1}
$$

for the concentration in the $\mathrm{N}^{\text {th }}$ mixing cell is:

where:

$$
I_{n+1}=\int_{0}^{t} d t_{1} \int_{0}^{t_{1}} d t_{2} \int_{0}^{t_{2}} \ldots \int_{0}^{t_{n}} d t_{n+1} Q_{N-n}(t) e^{(\alpha N+\lambda) t}
$$

Equation (A.26) applies only if the parameter alpha is constant.

The first term in Eqn. (A.25) arises from the initial conditions, $\left(C_{n}(0)\right.$ is the concentration in the $\mathrm{n}^{\text {th }}$ cell at time $\left.=0\right)$. In most instances, the initial concentration in the solute is expected to be zero.

The integral in Eqn. (A.26) can be evaluated analytically for certain functional forms of the wasteform release rate term, $\mathrm{Q}_{\mathrm{n}}$. In particular, rinse release and dissolution release (constant release rate) with radioactive decay can be modeled as follows:

$$
\begin{aligned}
& Q_{n}(t)=C_{n}\left(\tau_{n, b}\right) \delta\left(t-\tau_{n, b}\right) \\
& \quad+Q_{n}(0) e^{-\lambda t}\left[H\left(t-\tau_{n, b}\right)-H\left(t-\tau_{n, f}\right)\right]
\end{aligned}
$$


where:

$$
C_{n}\left(\tau_{n, b}\right)=\frac{M_{r, n} e^{-\lambda \tau_{n, b}}}{\theta V_{n} R}
$$

where:

$\mathrm{M}_{\mathrm{r}, \mathrm{n}}=$ the mass available for rinse release at $\mathrm{t}=0$;

$\tau_{\mathrm{n}, \mathrm{b}}=$ time of total failure of the $\mathrm{n}^{\text {th }}$ container;

$\mathrm{V}_{\mathrm{n}}=$ the volume of the $\mathrm{n}^{\text {th }}$ mixing cell (the volume of the entire

disposal facility divided by the number of mixing cells, $\mathrm{N}$ );

$\delta\left(\mathrm{t}-\tau_{\mathrm{n}, \mathrm{b}}\right)=\operatorname{Dirac} \delta$ function;

$\mathrm{Q}_{\mathrm{n}}(0)=$ release rate at time $=0$;

$\mathrm{H}\left(\mathrm{t}-\tau_{\mathrm{n}, \mathrm{b}}\right)=$ Heaviside function, defined as follows:

$\mathrm{H}\left(\mathrm{t}-\tau_{\mathrm{n}, \mathrm{b}}\right)=0 \quad \mathrm{t}<\tau_{\mathrm{n}, \mathrm{b}}$

$\mathrm{H}\left(\mathrm{t}-\tau_{\mathrm{n}, \mathrm{b}}\right)=1 \quad \mathrm{t}>\tau_{\mathrm{n}, \mathrm{b}}$

$\tau_{\mathrm{n}, \mathrm{f}}=$ completion time for wasteform release, i.e., no further release is permitted after this time. This can be calculated by taking the inverse of the yearly fractional release rate and adding this value to the time of breach.

The first term represents the instantaneous surface wash-off that occurs immediately after container breach when water first contacts the wasteform. The second term models a constant release rate corrected for first order (radioactive) decay which starts immediately after container breach and finishes at the time the entire inventory is released.

If $\mathrm{Q}_{\mathrm{n}}(0)$ is used to model a dissolution controlled process, it would be estimated as the product of the dissolution velocity, surface area of the wasteform, and the concentration of contaminant within the wasteform as discussed in the wasteform release section of Chapter 2.

As currently written, the release rate term, $\mathrm{Q}_{\mathrm{n}}$, is not directly applicable to modeling the monotonically decreasing release rate representative of diffusion. Including a diffusion term is conceptually easy. However, because the analytical solution to diffusion release is an infinite series (see the diffusion release section in this Appendix), the multiple integrations required in Eqn. (A.29) are cumbersome.

However, if the exponentially decaying release rate term is used to approximate release that is controlled by diffusion, $\mathrm{Q}_{\mathrm{n}}(0)$ would be estimated as the product of the diffusion coefficient, a geometric factor which involves the surface area, and the concentration in the wasteform. Using the wasteform 
release rate term given in Eqn. (A.27) in the analytical solution, Eqn. (A.25), yields the following solution for the concentration in the last mixing cell:

$$
\begin{aligned}
C_{N}(t)= & e^{-\lambda t} e^{-\alpha N t} \sum_{n=0}^{N-1} C_{N-n}(0)(\alpha N t)^{n} / n ! \\
& +\sum_{n=0}^{N-1} C_{N-n}\left(\tau_{N-n, b}\right) H\left(t-\tau_{N-n, b}\right)\left(\alpha N\left(t-\tau_{N-n, b}\right)\right)^{n} \bullet e^{-(\alpha N+\lambda)\left(t-\tau_{N-n, b}\right)} / n ! \\
& +\frac{\beta}{\alpha} e^{-\lambda t} \sum_{n=0}^{N-1} Q_{N-n}(0) H\left(t-\tau_{N-n, b}\right)\left(1-e^{-\alpha N\left(t-\tau_{N-n, b}\right)}\right) \\
& -\frac{\beta}{\alpha} e^{-\lambda t} \sum_{n=1}^{N-1} Q_{N-n}(0) H\left(t-\tau_{N-n, b}\right) e^{-\alpha N\left(t-\tau_{N-n, b}\right)} \sum_{i=1}^{n} \frac{(\alpha N)^{i}\left(t-\tau_{N-n, b}\right)^{i}}{i !} \\
& +\frac{\beta}{\alpha} e^{-\lambda t} \sum_{n=1}^{N-1} Q_{N-n}(0) H\left(t-\tau_{N-n, f}\right) e^{-\alpha N\left(t-\tau_{N-n, f}\right)} \sum_{i=1}^{n} \frac{\left(\alpha N\left(t-\tau_{N-n, f}\right)\right)^{i}}{i !} \\
& -\frac{\beta}{\alpha} e^{-\lambda t} \sum_{n=0}^{N-1} Q_{N-n}(0) H\left(t-\tau_{N-n, f}\right)\left(1-e^{-\alpha N\left(t-\tau_{N-n, f}\right)}\right)
\end{aligned}
$$

The above expression for $\mathrm{C}_{\mathrm{n}}$ is quite general and permits each mixing cell to model a container with a unique time of breach, inventory, and release rate. The first term in Eqn. (A.29) represents the initial condition. The second term represents the concentration in solution due to surface rinse beginning immediately after breach. The remaining four terms represent the concentration of radioactivity in solution due to a wasteform that releases mass uniformly in time adjusted for radioactive decay.

A number of different situations can be adequately modeled with Eqn. (A.29) through proper selection of the input parameters. However, because of the restriction that the wasteform release rate be expressed either by surface rinse or an exponentially decaying rate, the above expression can not be used to investigate localized failures because they lead to time-dependent wasteform release rates (as the failure area grows, the release rate increases).

The mass flux out of the disposal facility is the concentration in the last cell multiplied by the Darcy velocity. The total mass flow rate is the mass flux multiplied by the area of the disposal facility.

$$
M_{f}(t)=C_{n}(t) V_{d} A_{f}
$$

\section{$\underline{\text { Finite Difference Solution Procedure }}$}

If the assumptions of the mixing cell cascade model (constant water flow, uniform retardation and moisture content, advection controlled transport, and only catastrophic container failures (e.g., no localized failures)) are not justified, the finite difference (FD) solution procedure is 
recommended.

The FD model uses a control volume approach when performing the mass balance. Figure A. 2 presents a typical control volume discretization scheme. To define the system, the user supplies the number of positions at which the concentration is calculated, NNP, and the locations which are used to determine the control volumes, X(I), where I ranges from 1 to NNP. In Figure A. 2 the solid lines mark the locations defined through input. The dashed lines represent the control volumes. That is, the first concentration value is the average for the region contained by $X=X(1)$ and $X=1 / 2(X(2)-X(1))$; the second concentration value is for the region $X=1 / 2(X(2)-X(1))$ to $X=X(2)+1 / 2(X(3)-X(2))$; and so on. The average concentration can be viewed, although this is an approximation, as the concentration at the midpoint of the control volume. With this assumption, and for uniform spacing, the first concentration value represents the concentration at $(\mathrm{X}(2)-\mathrm{X}(1)) / 4$, the second represents the concentration at $\mathrm{X}(2)$, the third at $\mathrm{X}(3)$, etc. This pattern continues until the last control volume, where the concentration at the last computational point is approximately the concentration at the point given by $\mathrm{X}=\mathrm{X}(\mathrm{NNP})-1 / 4(\mathrm{X}(\mathrm{NNP})-\mathrm{X}(\mathrm{NNP}-1))$.

This indicates that the first and last concentration values are not boundary values. Boundary values are incorporated directly into the mass balance equation when calculating the first and last concentration values. This procedure is described later in this Appendix.

Although it is not explicit in Fig. A.2, the size of the control volumes may vary with location. For non-uniform spacing, the midpoint of the control volume will not coincide with the input locations. For example, if $\mathrm{X}(1)=0, \mathrm{X}(2)=1$, and $\mathrm{X}(3)=10$, control volume 1 will cover the range 0 to $1 / 2$, while control volume 2 is the region $1 / 2$ to 5.5 and has a midpoint of 3 . This can become important when interpreting results. 


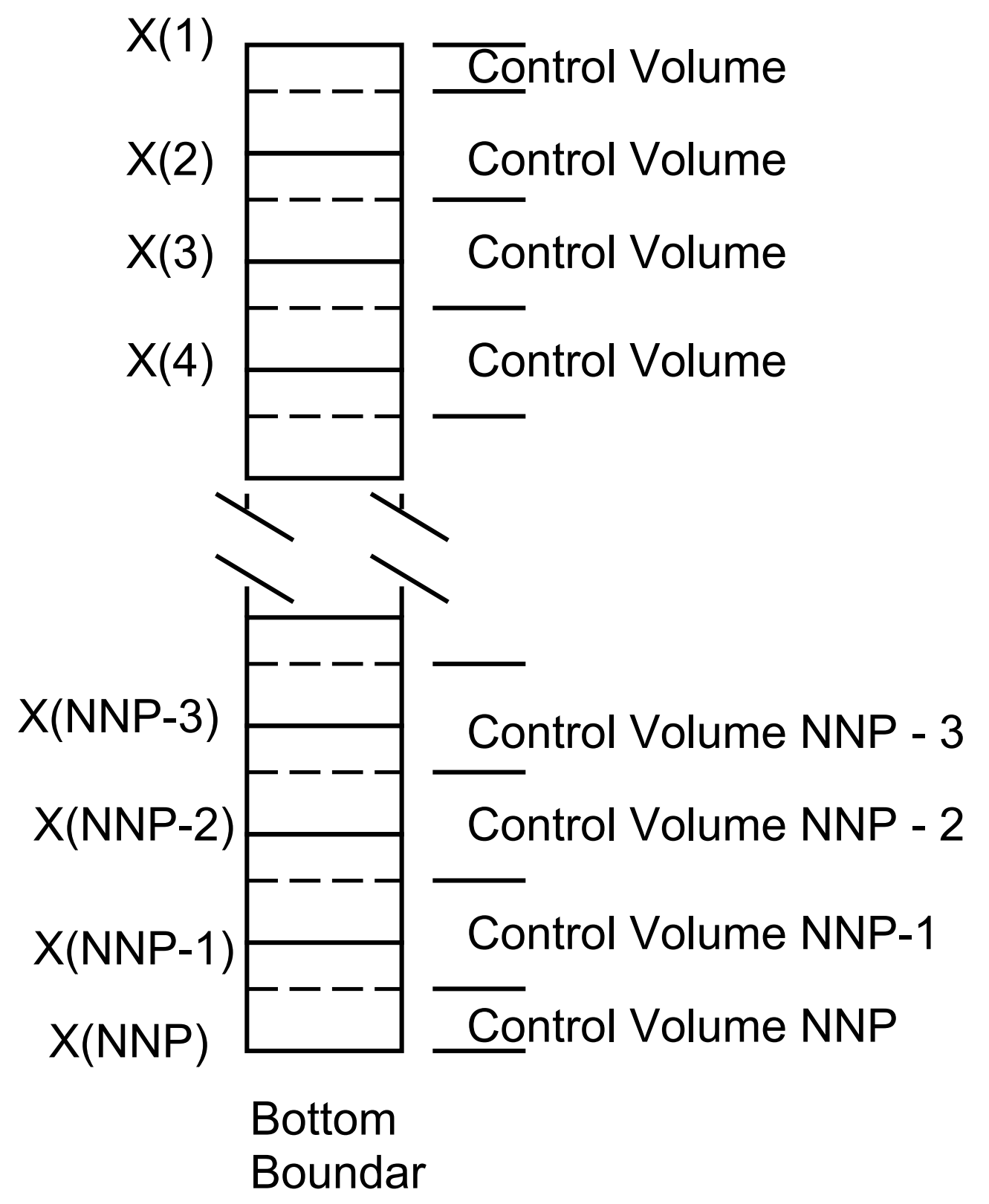

Figure A- 2 Control volume discretization of a modeled region.

In specifying containers, wasteform, and external source locations, the locations correspond to a control volume. That is, if a wasteform is placed in location 5 , it is in the region defined by control volume 5 .

The finite difference procedure begins with Eqn. (A.22). The second order derivative (the diffusiondispersion term) is estimated using centered differences. The first order spatial derivative is approximated using upwind differencing. The time derivative term is approximated using a first order 
backward difference. Performing this, results in the following equation:

$$
\begin{aligned}
& \frac{\left(\theta R C_{n}\right)^{i+1}}{\Delta t}=\frac{\left(\theta R C_{n}\right)^{i}}{\Delta t} \\
& +C_{n+1}^{i+1}\left[\frac{(\theta D)_{n+1}^{i+1}}{\Delta X_{n} \Delta X_{n, a}}\right] \\
& \quad-C_{n}^{i+1}\left[\frac{(\theta D)_{n}^{i+1}}{\Delta X_{n} \Delta X_{n, a}}+\frac{(\theta D)_{n}^{i+1}}{\Delta X_{n-1} \Delta X_{n, a}}+\frac{V_{D}^{i+1}}{\Delta X_{n-1}}+\lambda(\theta R)^{i+1}\right] \\
& +C_{n-1}^{i+1}\left[\frac{(\theta D)_{n-1}^{i+1}}{\Delta X_{n-1} \Delta X_{n, a}}+\frac{V_{D}^{i+1}}{\Delta X_{n-1}}\right]
\end{aligned}
$$

where $\Delta X_{n, a}=\frac{1}{2}\left(\Delta X_{n}+\Delta X_{n-1}\right)$

$+q_{n}^{i+1}$

where the superscript $i$ refers to the time level of the calculation and the subscript $\mathrm{n}$ refers to the spatial location of the calculation. Except for the time-derivative term, all concentrations are evaluated at the new time level, $\mathrm{i}+1$. This is known as the implicit solution procedure and insures that the numerical solution is stable, i.e. numerical errors that occur in solving the equation are damped.

Equation (A.31) applies for each control volume in the interior (n not equal to 1 or $\mathrm{N}$ ) of the domain being simulated. Equation (A.31) illustrates that the concentration in control volume $\mathrm{n}$ is a function of its two nearest neighbors. The system of equations which accounts for the concentration at each point can be rearranged such that all unknown variables at the new time level are on the left hand side of the equation and all known variables (the release rate and concentrations at the old time level) are on the right hand side. Performing this arrangement and using matrix notation yields:

$$
\overline{\mathrm{A}} \overline{\mathrm{C}}(\mathrm{i}+1 \overline{)}=\mathrm{S}
$$


where $\mathrm{A}$ is a tridiagonal matrix comprised of the terms that multiply the concentrations in each control volume at time level $i+1, \mathrm{C}$ is a vector that represents the concentration in each control volume at time level $\mathrm{i}+1$, and $\mathrm{S}$ is a source term comprised of the release rate from the wasteform over the time step plus the concentration at time level i. The system of equations represented by Eqn. (A.32) can be solved quite easily using standard numerical techniques.

\section{$\underline{\text { Boundary Conditions }}$}

Special consideration is given to the first and last control volume. These control volumes are modified to reflect the boundary conditions imposed on the problem. Typical boundary conditions are that the concentration or the flux is specified as a function of time. The boundary condition applied to the top of the disposal facility could be zero mass flux entering the facility. At the bottom of the facility, the concentration or the flux may be specified. Requiring the concentration at the bottom of the facility to be zero would lead to the highest mass flux out of the facility. Similarly, requiring zero mass flux out would lead to the highest solution concentration. In addition, the user can specify the advective and the diffusive/dispersive flux at the boundary. Time dependence of the boundary conditions is modeled through an input table of values versus time.

The mass balance equation for any control volume of width $\Delta \mathrm{X}$ is:

$$
\frac{\partial(\theta R C)}{\partial t}=-\left[\frac{J_{\text {out }}-J_{\text {in }}}{\Delta X}\right]-\lambda \theta R C+q
$$

where $\mathrm{J}_{\text {out }}$ is the mass flux at the downstream boundary (largest value of $\mathrm{X}$ ) and $\mathrm{J}_{\text {in }}$ is the mass flux at the upstream boundary.

For interior control volumes, the mass fluxes are evaluated using Eqn. (A.21). At the boundary control volumes, the boundary mass flux is incorporated directly into the equation based on the choice of boundary condition.

\section{$\underline{\text { First Control Volume }}$}

For the top boundary, e.g., the first control volume (Fig. A.2), the finite difference mass balance becomes:

$$
R_{l}\left[\frac{C_{l}^{i+1}-C_{l}^{i}}{\Delta t}\right]=-\left[\frac{J_{1}-J_{b}}{\Delta X / 2}\right]-\lambda R_{l} C_{l}+q_{1}
$$


where $\Delta \mathrm{X}=\mathrm{X}(2)-\mathrm{X}(1)$, the subscripts 1 refer to control volume 1 , and the superscript $\mathrm{i}$ refers to the time level of the calculation.

The boundary flux, $\mathrm{J}_{\mathrm{b}}$, is:

$$
J_{b}=-\theta D_{l}\left[\frac{C_{1}-C_{b}}{\Delta X_{1} / 4}\right]+V_{d} C_{b}
$$

This can be specialized for the four boundary conditions $(\mathrm{BC})$ as follows:

\section{BC 1: Specified Concentration}

The $\mathrm{BC}$ is:

$$
C_{b}=g(t)
$$

where $g(t)$ is specified in an input table.

In this case:

$$
J_{b}=-\theta D_{1}\left[\frac{C_{1}-g(t)}{\Delta X_{1} / 4}\right]+V_{d} g(t)
$$

BC 2: Specified Total Flux

This $\mathrm{BC}$ is:

$$
J_{b}(t)=g(t)
$$

BC 3: Specified Advective Flux

This $\mathrm{BC}$ is:

$$
V_{d} C_{b}=g(t)
$$

This is mathematically equivalent to specifying the concentration at the boundary. 
In this case:

$$
J_{b}=-\theta D_{I}\left[\frac{C_{1}-g / V_{d}}{\Delta X / 4}\right]+g(t)
$$

BC 4: Specified Diffusive/Dispersive Flux

This $\mathrm{BC}$ is:

$$
-\theta D \frac{\partial C}{\partial x}=g(t)
$$

Here,

$$
J_{b}=g(t)+V_{d}\left[C_{I}+\frac{4}{\theta D \Delta X} g(t)\right]
$$

In this case, the boundary flux depends on the concentration in the first volume element, $\mathrm{C}_{1}$. This reflects the link between the concentration at the boundary and in the first element due to diffusion/dispersion.

Depending on the choice of the BC, one of equations (A.37), (A.38), (A.40), or (A.42) is used directly in Eqn. (A.34) to generate the appropriate finite difference representation of the first control volume.

\section{$\underline{\text { Equations for the Last Control Volume }}$}

For the bottom boundary, e.g., the last control volume, (Fig. A.2), the finite difference mass balance becomes:

$$
R_{N}\left[\frac{C_{N}^{i+1}-C_{N}^{i}}{\Delta t}\right]=-\left[\frac{J_{b}-J_{N}}{\Delta X_{N} / 2}\right]-\lambda R_{N} C_{N}+q_{N}
$$

where $\Delta X_{N}=X(N N P)-X(N N P-1)$ in Fig. A.2, the subscript $N$ refers to the last control volume, and $i$ refers to the time level. 
The boundary flux, $\mathrm{J}_{\mathrm{b}}$, is:

$$
J_{b}=-\theta D_{N}\left[\frac{C_{b}-C_{N}}{\Delta x_{N} / 4}\right]+V_{d} C_{N}
$$

Here the advective flux is independent of the boundary condition. This is consistent with the upwind finite differencing procedure used to generate the previous control volume mass balance equations.

The four choices for boundary condition are used to calculate the boundary flux as follows:

\section{$\underline{B C}$ 1: Specified Concentration}

The $\mathrm{BC}$ is:

$$
C_{b}=g(t)
$$

where $\mathrm{g}(\mathrm{t})$ is specified through tabular input.

In this case:

$$
J_{b}=-\theta D\left[\frac{g(t)-C_{N}}{\Delta x_{N} / 4}\right]+V_{d} C_{N}
$$

BC 2: Specified Total Flux

The $\mathrm{BC}$ is:

$$
J_{b}=g(t)
$$

BC 3: Specified Advective Flux 
The $\mathrm{BC}$ is:

$$
V_{d} C_{b}=g(t)
$$

Here,

$$
J_{b}=-\theta D\left[\frac{g(t) / V_{d}-C_{N}}{\Delta x_{N} / 4}\right]+V_{d} C_{N}
$$

Again, this is mathematically equivalent to specifying the concentration. In general, at the last boundary unless the advective flux is zero, it will be difficult to specify a priori. Further, it may lead to an inconsistency in the interpretation of the boundary advective flux. In the mass balance equation, Eqn. (A.43), the advective flux is $\mathrm{V} \mathrm{C}_{\mathrm{N}}$. However, the boundary condition advective flux is given as $g(t)$.

BC 4: Specified Diffusive/Dispersive Flux

The $\mathrm{BC}$ is:

$$
-\theta D \frac{\partial C}{\partial x}=g(t)
$$

In this case,

$$
J_{b}=g(t)+V_{d} C_{N}
$$

Depending on the choice of boundary condition, one of equations (A.46), (A.47), (A.49), or (A.51) is used in Eqn. (A.44) to generate the appropriate finite difference representation of the last control volume.

\section{REFERENCES}


[Kozak, 1990]

M.W. Kozak, et. al., Background Information for the Development of a Low-Level Waste Performance Assessment Methodology: Computer Code Implementation and Assessment," NUREG/CR-5453, SAND89-2509, Volume 5, Sandia National Laboratories, 1990.

[Pescatore, 1991]

C. Pescatore, "Leach Rate Expressions for Performance Assessment of Solidified, Low-Level Radioactive Waste," accepted for publication by the Journal of Waste Management, also available as an informal report, WM-3276-12, Brookhaven National Laboratory, December, 1990. 


\section{Appendix B: DUSTWIN - Windows based DUST pre-processor}

\section{B-1.0 Introduction to DUSTWIN for Windows}

A Windows based input pre-processor has been developed to facilitate creating an input deck for the DUST and DUST-MS computer codes. DUSTIN for Windows (DUSTWIN) is a Visual Basic program that guides the user through all of the necessary steps to create an input deck. It can convert DUST input decks to DUST-MS input decks and vice versa. The output of DUSTWIN is identical to the DOS based versions of the code described in this report. However, the ability to use a mouse to navigate through the different menus and the visual display make the code somewhat easier to use. In addition, DUSTWIN permits the user to run the codes, graph and print the results all under one frame work.

This description on the application of DUSTWIN will focus on creation of a DUST-MS input deck. Detailed discussions of the definitions of the input parameters are presented in the sections on the DOS based version of DUSTIN and the documentation for creating a DUST-MS input deck and will not be repeated in this section.

\section{B-2.0 Main Menu}

Upon installation of DUSTWIN it is recommended to create a shortcut key. To run the code, select the short cut key and either double click the left mouse button or hit the return key. In the remainder of this description instructions will be given in terms of using the mouse.

Once DUSTWIN has been started the main menu appears across the top of the screen with the following choices:

\section{File Model type Run DUST Graph Output Editors Help}

These choices permit creation of a new input deck or modification of the old input deck, execution of the DUST codes, graphing of selected output results, and access to editors to review or print the input or output data The following sections describe the major options within each of these submenus.

\section{B-2.1 File Option}

The File option allows the following choices:

New Create a new input file

Open Open an existing input file for modification. When this option is selected, another window opens which permits the user to change the drive or directory. Files in the current directory are listed and can be selected by appropriate use of the mouse and keyboard.

Save File Save file with the current path, name, and extension. If this is a new file which has 
not been named, the user is prompted for a file name.

Save File As Save file with a new path, name, or extension.

Print File Prints the current input file to the default printer.

Exit Closes the program and returns to Windows.

SAVE FILE

After making the desired changes to an existing input deck, or creating a new input deck, the file must be saved. Use of the Save or Save As option creates a permanent file. This is a necessary step to perform prior to running the code. When running the code from the DOS shell, the file used as input is the most recently saved version of the file. Failure to save the file prior to running the code will cause the old file to be used for input and provide unintended results.

\section{B-2.2 Model Type Option}

The input requirements are slightly different for the DUST (single species) and the DUSTMS (multiple species) codes. Therefore, the user must select which type of input file will be created. The default file type is DUST-MS. The Model type option specifies the choice of DUST or DUST-MS. If the user wishes to use the DUST code, the model type must be switched prior to reading an existing input deck or creating a new input deck. To assist the user in determining which type of input file is being created, the background screen is set to a different color for each code, blue for DUST-MS and pink for DUST. In addition, the choice of code is written across the top of the screen.

Taking an existing input deck and switching between Model type will convert the code to the newly selected model to the best of its ability. That is, for translating multiple species input decks to single species, only information pertaining to the first species is retained. Similarly when going from the single species DUST code to the multiple species DUST-MS code, information is provided for one species only. This approach is not recommended. It is best to define either a DUST or DUST-MS model type and use it exclusively.

\section{B-2.3 File Type Option}

The file type option provides the following choices:
DUST
DUST-MS
DUSTAQIN: CREATE BOUNDARY CONDITION FILE
Run Source Term and Aquifer Simulation Sequentially 
The File Type option specifies the choice of DUST (single species source term model), DUST-MS (multiple species source term model), DUSTAQIN. (Post-processor that takes DUST-MS trace file output and creates a boundary condition file), or Run source term and aquifer simulation sequentially (uses a DOS batch file, DUSTAQ.BAT, to simulate release from the facility and flow in an aquifer by running DUST-MS twice, once for the facility and unsaturated zone, and once for the aquifer. This procedure begins with use of DUST-MS to obtain the flux from the repository entering the aquifer, it then runs DUSTAQIN to create the boundary condition file of the mass flow rate into the aquifer, finally, it runs DUST-MS again to simulate transport in the aquifer). When running DUSTAQ from the Windows 95 pre-processor, both input files must be created prior to the run. Upon selecting one these choices, DUSTWIN opens a DOS shell, runs the selected code, and returns to DUSTWIN upon termination of the run. Since, it opens a DOS shell, it is independent of the settings within DUSTWIN. For example, even if a DUST-MS file is selected in the Windows Pre-processor, the user can run the DUST code by selecting it from the File Type option sub-menu. In the DOS shell, the file to be used for input must be typed similarly to running from DOS. If the default path defined in DUSTWIN is different than the location of the data files, the entire path must be specified. If this is not done, a file not found error message will be returned.

\section{B-2.4 Graph Output Option}

The graph output option provides the following choices:

\section{GRAFXT (DUST) GRAFMS (DUST-MS)}

GRAFXT is a DOS program which plots information (fluxes, mass flow rate, total mass passing a fixed point, or concentrations) for DUST "trace output files." GRAFMS is the counterpart for DUSTMS. These files search for the trace files. If a message occurs that the file is not found, please make sure that the executable files are in the same directory in which the simulation was run.

\section{B-2.5 Editors Option}

The editors option menu provides the following choices:

\section{NotePad \\ WordPad \\ DOS Edit \\ Other Editor}

The installation of DUSTWINprovides a file, EDITPATH.TXT, which contains the path to the above listed editors. Default paths typical for Windows '95 are supplied for the first three editors. The executable file name and path must be supplied for the other editor. The first three editors are all ASCII editors and can be used to modify input files created for DUST or DUST-MS. NotePad is limited in that it can only accept small files (less than approximately 0.5 Mbyte). WordPad does not have this limitation and is useful for viewing large output files. DOS Edit has the advantage of allowing multiple files to be open simultaneously. This is useful when comparing files. All options within these editors (edit, print, open, save, etc) are available to the user. The Other Editor option is 
provided to allow flexibility to open more commonly used word processing files such as Word or WordPerfect..

\section{B-2.6 Help Option}

The Help option lists the name and phone number of the code authors as a point of contact for problems in the operation of the code. A full on-line help menu is planned but has not been developed at this time.

\section{B-3.0 Input Data}

In addition to the options menu at the top of the page, a data set menu is listed vertically at the left of the screen. The data set menu contains ten buttons to define each of the ten data sets needed for DUST-MS. The buttons are:

Title: $\quad$ Define problem title and miscellaneous parameters such as the number of isotopes, isotope half-lives, solubility limits, mass activity flag, and the number of nodal points,

Time Define time step parameters.

Material Define the number of sets of material properties and their values.

Output Define output times, trace locations and times.

Facility Dimensions Define node spacing and facility area.

Initial and BCs Define the initial conditions for each isotope at each nodal point and the boundary conditions for each isotope at the two boundaries.

Water Flow

Container Waste Form Define tine-dependent water flow rate, space-dependent moisture content

Sources Define container performance parameters and container locations Define waste form performance parameters Define time-dependent sources and their locations.

To activate one of the buttons, place the arrow on the appropriate button and click with the left mouse button. For the most part, the use of DUSTWIN is self explanatory and follows standard Windows conventions. The following sections will describe the input in more detail highlighting areas that may be confusing to the new user.

\section{B-3.1 TITLE}

Upon accessing the Title dialog box, DUSTWIN searches for the file RNUCL.DAT which contains a list of over 200 radionuclides with their half-lives, default solubility limits $\left(10 \mathrm{~g} / \mathrm{cm}^{3}\right)$, atomic mass, and name (e.g., U-238). If this file is not found, a menu appears asking to search the disk for the file. The easiest approach to find the file is to go to the section in the lower left corner, Start Search Directory, move the mouse to $C: \backslash$, the root directory, and double click. This should list all directories on the $\mathrm{C}$ drive. Next, go to the begin search button and click. A list of all occurrences of the file will appear. Go to the appropriate file name and select it. Then, go to the select file path button and click on it. This path is stored by DUSTWIN and will be used in subsequent uses of the code. 
After finding the RNUCL.DAT file, a menu appears with the following choices:

Title:

An alpha-numeric string used for problem identification

Number of Nodes The number of finite difference nodes

Number of Isotopes Must be less than 10.

Mass Units

Model Type
Input in Curies Becquerels, or grams.

Mixing Cell or Finite Difference. In DUST-MS, the user is not permitted to select the mixing cell option.

OK Returns to the main menu and saves all changes.

\section{Cancel}

In addition, for each isotope, there is an input box for the isotope name, atomic weight, half-life, and solubility limit. These can be input through the keyboard, or by selection from the pull down list of radionuclides supplied at the right of the menu. To use the pull down list, position the mouse under the column titled isotope on the menu and click. This leaves the cursor at this point. Then take the mouse to the pull down menu, select the appropriate radionuclide and click on it. This will insert all of the data (isotope name, atomic weight, etc.) in the appropriate box. To define the next radionuclide, return to the isotope column, click on the appropriate row of the isotope that is being defined and repeat the selection process. If the step to redefine the isotope that is receiving changes is omitted, the code will overwrite the existing values. In general, if values exist and a new isotope is selected, the values are overwritten. To clear the definition of the isotopes, a button titled clear is provided After selection of isotope parameters through the pull-down menu, any parameter can be redefined by moving the cursor to the appropriate input box and typing in a new value. When typing in new values, typed in values are inserted at the cursor location. Caution should be used to insure that the existing values are deleted. For example, if an existing value of 0 is to be changed to 1 , moving the cursor to right before the zero and typing 1 will leave a value of 10 . The zero must be deleted. This is a common mistake when using DUSTWIN which should be monitored.

After completion of the first menu, a second menu appears which allows definition of decay chains. The default is zero decay chains. If decay chains are simulated, click on the arrow for the input box for the number of decay chains and select the number of decay chains. The default value for the number of members in a chain is two. This can be changed using the same procedure as above. The code assumes that species one decays to species two. To change this, click on the appropriate row in the column labeled members and then click on the appropriate choice from the isotope list. A check in the code prevents an isotope from appearing more than once in a decay chain. The fraction of decays that lead to the progeny in the chain can also be reduced from the default value of 1 . This permits simulation of branching.

Once the decay chains have been defined, click the OK button. To return to the main menu without saving, click on Cancel.

\section{B-3.2 TIME}


Parameters that define the time steps are found in this data set and they include:

Number of time steps in the simulation

Initial time step (in years)

Fractional change in time step

Maximum time step size

Maximum simulation time

Number of time step resets

Time (in years) at which the time step is reset to the initial time step.

All of these values can be changed by moving the cursor to the appropriate input box and typing in the desired number. To return to the main menu, click on OK (saves changes) or Cancel (changes not saved).

\section{B-3.3 MATERIAL}

This menu permits definition of the number of different material types, the number of changes in material type, and the material properties needed to assess transport from the disposal facility. In DUST-MS a material is designated for each region with unique transport properties $(\mathrm{Kd}$, diffusivity, diffusion coefficient, and bulk density). The user is permitted to display the transport parameters by material (i.e. material properties for a single material for all isotopes) or isotope (i.e., material properties for each material for a single isotope). Parameters can be defined by positioning the cursor in the appropriate box and typing in a value.

It should be noticed that not all properties for all materials are displayed on the screen at any given time. For example, if there are two materials three isotopes, and the display is set to by material type, the menu will display all transport properties for each isotope for a single material. To define the transport properties for the second material, the value in the "values for material" box should be changed from 1 to 2 . Similar remarks apply if the display is by isotope. In this case, all values for the selected isotope are displayed, to define other isotopes, the value in the "values for isotope" box needs to be changed.

Once all material properties for all isotopes have been defined, click on the OK button. If the number of material changes is greater than 0 , a menu pops up asking for a definition of the nodes that are to be given a material type different than the default value of 1 . For large regions containing the same material type, they can be defined by specifying the first and last nodes in the region and the material type for that region.

\section{B-3.4 OUTPUT}

The first menu in the output segment of the code asks for a definition of the times at which output is requested. In DUST and DUST-MS output is controlled by a flag that has a value at every time step. A value of 0 implies no output at the time step. A value of $1-4$ specifies varying amounts of output as described on the menu. 
DUSTWIN limits the number of output flags to the maximum number of time steps specified in Data Set 2 and displays the output flags in groups of 25. If more than 25 time steps are specified, the user must select which group of 25 to view. To facilitate output at regular time step intervals, the code allows the user to define a series of output times through specifying the first and last time step in the series, the interval between output, and the output flag. For example, to print the concentration, output type 1 , every 10 time steps, the user would specify the following:

First step in series: $\quad 10$

Last step in series: $\quad 100$

Increment in step number: $\quad 10$

Initial Output type in series $\quad 1$

Change in output type 0

The DUST-MS code permits an unlimited number of time steps but only allows 1000 output times to be specified. If more than 1000 time steps are specified, the code automatically repeats the cycle of the first 1000 time steps. For example, assume that there are 2000 time steps requested for the simulation. The user specifies output at the $250,500,700$, and 1000 th time steps. The code will provide output at the $250,500,700,1000,1250,1500,1700$, and 2000 th time steps.

After completing this menu, DUSTWIN asks for the number of concentration trace variables requested, the number of time steps between output to the trace files, and the trace locations. This procedure is repeated for the flux trace variables.

\section{B-3.5 FACILITY DIMENSIONS}

The first menu asks for the area of the facility. This value is used to normalize the concentrations to the initial inventory and is needed because of. the one-dimensional representation of the facility. After clicking on OK, a menu appears to define the nodal locations. The input parameters requested are the first and last node in the series, the starting location of the first node, the change in position (delta $\mathrm{x}$ ), and the fractional change in node size.

If the mesh is uniform, the default parameters are set to permit the user to define only the change in position. The default values for the first node is one, the last node is the number of nodal points defined in data set 1 , the increment in node number is 1 , and the starting position is $0.0 \mathrm{By}$ defining delta $X$ as 100 , a uniform mesh of $100 \mathrm{~cm}$ regions would be defined. Non-uniform meshes can be defined by using multiple series.

The code checks that all nodal points have a unique defined location. If this is not the case, when attempting to exit using the $\mathrm{OK}$ button, an error message is printed and the user is requested to make changes to solve the problem.

\section{B-3.6 INITIAL AND BCs}

The first menu permits definition of the initial conditions. Upon entering this menu, the default value of zero concentration everywhere is defined. If this is inappropriate changes can be made 
through series definition similar to when defining nodal coordinates. Also upon entering, the initial conditions are displayed only for the first isotope. To define initial conditions for other isotopes, change the isotope specified in the "value for isotope" input box. The default value of zero initial concentration applies to all isotopes.

After defining the initial conditions for all isotopes, click the OK button. This causes the code to store initial conditions for all isotopes and presents a menu for defining boundary conditions. If the initial conditions are incorrect, the user must return to the main menu and re-enter this section.

The boundary condition menu permits definition of the time-dependent top and bottom boundary conditions for each isotope. Therefore, for three isotopes, six boundary conditions are required. To switch between these six boundary conditions two flags must be set. They are "values for isotope" and "values for upper boundary and lower boundary". The upper boundary always corresponds to node 1 while the lower boundary always refers to the last node.

The default boundary condition is zero concentration for all times at all boundaries. This is accomplished by having a table with an initial time of 0 , a final time equal to the maximum problem simulation time, and a value of zero at both times in the table.

Specification of the total flux, advective flux (Darcy velocity multiplied by concentration), and dispersive flux are also permitted. Time-dependence is specified by input of a table of the boundary condition value at specified times. Up to 10 data points can be used to define the time history at the boundary. Linear interpolation is used to calculate the boundary condition at simulation times not in the table.

The top boundary condition for each isotope may be read from an auxiliary file. This is accomplished by setting the $\mathrm{BC}$ file flag to yes. In this case, the data supplied above is overwritten by the data from the auxiliary file. This feature was developed to simulate the transfer of contaminant from the unsaturated zone to the aquifer. DUSTMS can be executed for the unsaturated zone creating an output file for the flux at the bottom of the unsaturated zone (FLUX trace file). This flux trace file is processed by the code DUSTAQIN.EXE which takes the mass flow rate leaving the unsaturated zone. The mass flow rate is translated to an aquifer flux by dividing by the area perpendicular to the flow direction specified by the variable AFACIL. In this case, DUST-MS permits specification of total flux only. Other boundary conditions (concentration, advective flux, or dispersive flux) are physically inapproriate.

\section{B-3.7 WATER FLOW}

This menu assists in the definition of water flow velocities and moisture contents in the modeled domain. Water flow is assumed to by spatially uniform but variable in time. The moisture content is assumed to be constant in time but variable in space.

The Darcy velocity is specified through tabular input of the velocity at specified times. Linear interpolation is used for times not in the table. The default value for water velocity is zero at all times. This can be changed as necessary. When inputting water velocities, the units are $\mathrm{cm} / \mathrm{s}$. Therefore, 
typical values are quite small being on the order of $10^{-7} \mathrm{~cm} / \mathrm{s}$. This may be input using exponential notation, e.g., $1 \mathrm{E}-7$.

The default value for moisture contents is 0 at all locations. This must be changed to a nonzero value because division by the moisture content occurs in several places in the code. Specification of the spatial distribution of moisture contents is similar in structure to definition of nodal locations or initial conditions.

\section{B-3.8 CONTAINER}

This menu asks for the number of containers, the number of failure types and the failure times for each container. Failure times may be defined by moving the cursor to one of the failure time boxes and typing a value or by defining a series of failure times. When editing a series of failure times, the input requested is the first and last container to be defined, increment in containers being defined, initial failure time in the series, the increment in failure time, and the fractional change in the increment in failure time. As an example, to define the first 10 containers to fail 10 years apart starting at 50 years, the user would input:

First container in series: 1

Last container in series $\quad 10$

Increment in container Number $\quad 1$

Initial failure time $\quad 50$

Increment in failure time 10

Fractional change in failure time $\quad 0$

To define all containers in a series to fail at the same time, the increment in failure time is set to zero.

After the failure times are defined, the location of all containers must be specified. This is accomplished through a menu similar to defining the container failure times. The requested input is the first and last node to be defined, increment in container number, node number of the location of the first container in the sequence, and the increment in the node number of the container location.

After specifying the container locations, a menu appears asking for the failure mode. If general failure is the only failure mechanism, further input is not required. If localized corrosion is to be modeled, the menu expands to list the additional input requirements. These are the thickness of the container, the pitting parameters $\mathrm{N}$ and $\mathrm{k}$, the area of the container, the area scaling factor, and the number of penetrating pits for each container. Definition of these parameters and guidance for selecting them is supplied in the DUST Data Input Guide, NUREG/CR-6041.

\section{B-3.9 WASTE FORM}

This section of the code defines the waste form release rate parameters, waste form type, and the initial inventory for each isotope. A waste form type defines a unique set of release rate parameters for each isotope. Therefore, for a problem with four waste form types and three isotopes, twelve sets of release rate parameters are required. These twelve sets are selected by changing the "value for 
isotope" and "value for waste type" selections through each combination.

DUST-MS provides six different release rate modeling options. The geometry can be rectangular, cylindrical, or spherical and releases can be simulated through analytical models or a finite-difference representation. The selection of the model is achieved by selecting the solution procedure and geometry in this menu. This choice can vary with waste type. For example, waste type one could be a cylindrical waste form using analytical solution procedures and waste type two could represent a spherical waste form using the finite difference procedure.

In addition to the geometry flag, the dimensions of the waste form must be specified. For rectangular geometry, the half-height, half-width, and volume are needed to completely define the waste form. For cylindrical geometry, the radius and volume are required. For spherical geometry, the radius fully defines the waste form.

The release rate parameters are the fraction of mass assigned to the rinse, diffusion, and dissolution (general degradation) release models and their rate parameters, partition coefficient, diffusion coefficient, fractional release rate, respectively. These six parameters must be defined for each waste form type and each isotope in the simulation prior to clicking on the OK button and proceeding to the next menu. If any of these parameters are not defined default values which may not be appropriate will be assigned.

The next menu requests the inventory in each container for each isotope. The default inventory is zero in all containers. Each isotope may have a unique inventory in each container. The inventories may be defined directly by positioning the cursor in the appropriate box and typing the value or by using the series definition similar to defining nodal locations. The units for the inventory are either grams or curies depending on the mass activity flag set in data set 1 .

After defining the inventory for each isotope, the waste type assignment menu appears if there is more than one waste type. In this menu, each container is given a unique waste type which specifies the release rate parameters to be used for that container. Waste type assignment can be achieved through positioning the cursor in the appropriate box and typing, or by defining a series as done with the inventory.

\section{B-3.10 SOURCES}

Selection of this menu item allows definition of external sources. The default value is zero external source for each isotope and changes to the input are not required if this is the case being simulated.

To define external sources the number of source nodes must be greater than zero. After setting a non-zero value for the number of source nodes, the menu expands to include the other parameters needed to define the source strength as a function of time. These parameters include the number of points in the table of source strength versus time, the number of unique source strength tables and the source strength table. This table is called the source profile. Each radionuclide can have up to eight unique source profiles. Again care must be exercised to insure that all variables are defined as desired 
prior to hitting the OK button and moving on to the next menu. This menu defines the source profiles for all radionuclides. Therefore, if there are three radionuclides each with two profiles, all six profiles should be defined before proceeding to the next menu. Profiles are defined by selection of the isotope and "values for profile".

The next menu defines the locations for the sources. Each isotope may have unique source locations. Definition can occur by moving the cursor to one of the boxes and typing an integer between 1 and the maximum number of nodal points in the simulation, or, a series of locations can be defined in a procedure similar to defining container locations. Locations for each isotope with sources are defined through this menu. If the number of external source nodes for an isotope is zero, no definition is required and the code does not permit selection of that isotope.

If there is more than one source profile, assignment of a profile to each source node must occur. The default value is source profile 1 at all locations. To change this, move the cursor to the appropriate box and type in the new source profile. A series of source types can be defined using a procedure similar to that used in defining container and waste types.

\section{B-4.0 AUXILIARY FILES}

DUSTWIN uses two auxiliary files, DUSTPATH.TXT and EDITPATH.TXT.

DUSTPATH.TXT contains a list of directories (paths) for the various files used by DUSTWINand has the structure:

\section{Path}

path $1 \backslash$ filename 1

path2 2 filename 2

path $3 \backslash$ filename 3

path4 \filename 4

path $5 \backslash$ filename 5

path 6\filename 6

path $7 \backslash$ filename 7
File used by DUSTWIN

RNUCL.DAT file with radionuclide dat

DUST.EXE

DUST-MS.EXE

GRAFXT.EXE

GRAFMS.EXE

DUSTAQIN.EXE (creates boundary condition files)

DUSTAQ.BAT (runs source and aquifer simulation sequentially).

For example, if the DUST-MS.EXE file is in the directory C:Idust, path3 should be set to C:Idust. The default path name is $\mathrm{C}: \mathrm{l}$. The first time a file is required by DUST-W95, the code opens the DUSTPATH.TXT file and searches the specified path. If the file is not found, a file search menu appears. It contains the file specification (i.e. file name), drive to search, the starting directory to conduct the search, a search button, and an accept file path button. It is best to start from the root directory. This can be accomplished by double clicking on the symbol for the root directory in the starting directory menu. After this occurs, click on the search button. A list of files that meet the file specification appears. As follows:

C: ldust

dust-ms.exe 
Click on the file name, not the directory and then click on the Accept file path. The file path is now stored for all subsequent uses of the DUSTWIN pre-processor. The paths may be edited using any standard ASCII word processor if desired.

The EDITPATH.TXT contains the file paths for the editors. The default settings are as follows.

\section{Path}

C:IWINDOWS $\backslash$ NOTEPAD.EXE

C:IProgram Files $\backslash$ Accessories $\backslash$ WORDPAD.EXE

C:IWINDOWS $\backslash$ COMMAND $\backslash$ EDIT.COM

C:I $\underline{\text { File }}$

NotePad

WordPad

DosEdit

Any Editor

If these file paths are not applicable, they can be changed either through the DUSTWIN pre-processor 


\section{APPENDIX C: FLOW CHART FOR THE DUST-MS CODE}

The DUST code contains 17 subroutines. This appendix provides a brief description of each subroutine and the organization of the computer code.

The main program is called DUST and it contains the dimension statements and common blocks for all of the primary variables. The DUST program calls the subroutine GM.

$\mathrm{GM}$, general manager, is the shell that controls all program operations. Initialization of key variables, calling the input routine, DATAIN, controlling the choice of transport model (Finite Difference (FD) or Multi-Cell Mixing Cascade (MCMC)), determining when output is required, and advancing the calculation in time are all performed by GM.

The subroutine DATAIN reads in all input parameters. As discussed in chapter 7, the input deck is divided into 10 data sets. Each data set contains the parameters required by the models used in the code, that is, one data set contains all the wasteform release parameters, etc. To facilitate the need for inputting a large number of parameters DATAIN relies on five auxiliary input subroutines: INPTR, INPTR2, INPTI, READR, and READN.

The INPTR subroutine is used to read in an array of real numbers. The DUST code reads the arrays: TIMOUT (output times for the MCMC model or time step change times for the FD model); TFAIL (time of total container failure); and WTINIT (initial mass in each wasteform) using INPTR.

The INPTR2 subroutine is used to read in two-dimensional arrays of real numbers which form the interpolation tables for boundary conditions, darcy velocity, and external sources. These arrays include: TBC (time of the boundary condition for each boundary); FBC (value of the boundary condition at the specified times for each boundary); TVDAR (time of the darcy velocity data points); FVDAR (value of the darcy velocity at the specified times); TSOSF (time of the source for each source profile); and SOSF (value of the source at the specified time for each profile).

The INPTI subroutine is used to read in arrays of integer numbers. The arrays include: NTRCEC (nodes for concentration traces); NTRCEF (nodes for flux traces); IPIT (pitting corrosion flag used in the FD model); NELCON (identifies the nodes with a waste container); ICTYPE (flag defining the container type in the FD model, that is, the DUST code permits several container types each with a unique set of localized failure parameters, this flag connects each container with a set of failure parameters), IDIFF (geometry flag for calculating diffusion-controlled releases in the FD model); IWTYPE (flag defining the wasteform type); LSRC (location of external sources); and ISTYPE (flag defining the type of source).

The READR subroutine is used to generate a sequence of values for selected real arrays. READR is used to define the node locations (X) in the FD model, the initial conditions for both transport models, and the moisture content at each location in the FD model. 
The READN subroutine is used to generate a sequence of values for the integer array IMAT which contains the material properties index when more than one material is used (FD model only).

The exact format and parameters required for each of these input routines is described in detail in Chapter 7 of the main body of this report.

Upon completion of the input routine program control is returned to subroutine GM. GM advances the time, calls the subroutine INTERP to calculate the value for external sources at the new time and checks to determine if the FD or MCMC transport model has been selected.

If the FD model is selected the subroutine BREACH is called. BREACH calculates localized failure rates and breached area as well as checks if the time to total failure has been exceeded. After failure occurs, material is released from the wasteform as calculated in subroutine LEACH. This release rate is fed into subroutine FDASSM which assembles the finite difference matrix used to calculate the migration of the radionuclides. FDASSM calls subroutine BOUND which incorporates the specified boundary conditions directly into the matrix. Upon completion of the generation of the matrix, the subroutine FDSLV, is used to solve the tri-diagonal matrix equation giving the concentration at the new time level at each location.

If the MCMC model is selected, the subroutine MIXBATH is called. MIXBATH checks if the time to failure of each container has been exceeded. If failure occurs, MIXBATH calculates the release rate from these containers and solves the analytical expression for transport of radionuclides through the facility.

After completion of the calculation of the concentrations, checks are made to determine if output is requested at the current time step. Flux and concentration traces are written to separate files within subroutine TRACE. The primary output file is written by subroutine PRINTT.

After output has been completed, subroutine GM advances the time variable and the procedure is repeated until the maximum number of time steps or maximum problem simulation time are exceeded.

Although subroutines FLUX and INTERP are located between subroutines TRACE and PRINTT in the flow chart, these subroutines are called several times during the calculation procedure. In particular, subroutine FLUX which calculates the total flux at every point is called prior to entering TRACE or PRINTT. Subroutine INTERP, which interpolates from a table, is used to calculate external sources, darcy velocity, and boundary conditions at the current simulation time. 


\section{APPENDIX D: DATA REQUIREMENTS FOR THE PITTING MODEL IN SUBROUTINE BREACH}

The container degradation models require information on the thickness and corrosion rates of the container. For 55 gallon carbon steel drums, typical thicknesses range from 50 - 60 mils, 0.127 - 0.152 $\mathrm{cm}$.

For the pitting model, five parameters are required: the rate constants $\mathrm{k}$ and $\mathrm{n}$, the area of the container, the area scaling factor, and the number of penetrating pits in the container.

The values of $\mathrm{k}$ and $\mathrm{n}$ for carbon steel have been determined from the National Bureau of Standards, NBS, data on corrosion in soils. $\mathrm{k}$ was found to range from $0.03-0.15 \mathrm{~cm} / \mathrm{yr}^{\mathrm{n}}$ with a mean value of $0.074 \mathrm{~cm} / \mathrm{yr}^{\mathrm{n}}$. $\mathrm{n}$ was found to range from $0-0.92$ with a mean value of 0.39 . A list of values for $\mathrm{k}$ and $\mathrm{n}$ in 47 different soils can be found in Romanoff [Romanoff, 1957]. This list has been ordered by soil aeration and reproduced in the previous Source Term topical report [Sullivan, 1988]. k was found to be correlated with soil $\mathrm{pH}$. $\mathrm{n}$ was found to be strongly correlated with the degree of soil aeration. The poorer the aeration, the higher the value of $n$.

The data base for stainless steels was insufficient to calculate the pitting parameters $\mathrm{k}$ and $\mathrm{n}$. After 14 years, often there was no measurable pitting.

The area of a 55 gallon drum is $2.1 \mathrm{E} 4 \mathrm{~cm}^{2}$. For other waste forms, this model requires the surface area in $\mathrm{cm}^{2}$.

The area scaling factor has been measured by Logan [Logan, 1939] for wrought iron pipes buried in 47 different soils. Values ranged from 0.08 - 0.32 with a mean value of 0.149 and a standard deviation of 0.042 . Table C-1 presents this data grouped by degree of soil aeration. The scaling factor did not exhibit a significant dependence on degree of aeration.

The number of penetrating pits has been estimated as 0.05 per $\mathrm{cm}^{2}$. DUST requires the number of penetrating pits per container. Thus, for a 55 gallon drum with a surface area of $2.1 \mathrm{E} 4 \mathrm{~cm}^{2}$, this implies 1050 penetrating pits.

The general corrosion rate for carbon steels and stainless steels has also been obtained from the NBS corrosion data in soils [Romanoff, 1957]. For carbon steels, these rates are presented in Table C2 and ranged from $2.7 \mathrm{E}-11-6 \mathrm{E}-10 \mathrm{~cm} / \mathrm{s}$ in a sample of 15 different soils. The mean value was $1.8 \mathrm{E}-$ $10 \mathrm{~cm} / \mathrm{s}$ and the standard deviation was $1.5 \mathrm{E}-10 \mathrm{~cm} / \mathrm{s}$. These values are the average rate for the duration of the experiment, 8 - 14 years. It was often noted that corrosion proceeded at a high initial rate and slowed down in time. 
In the same soils, for 304 stainless steel, the average corrosion rate was $8.9 \mathrm{E}-14 \mathrm{~cm} / \mathrm{s}$ with a standard deviation of $1.6 \mathrm{E}-13 \mathrm{~cm} / \mathrm{s}$. The range of measured corrosion rates was $5.3 \mathrm{E}-13-3.5 \mathrm{E}-15$ $\mathrm{cm} / \mathrm{s}$. For 316 stainless steel, the average corrosion rate was $4.1 \mathrm{E}-14 \mathrm{~cm} / \mathrm{s}$ with a standard deviation of $4.9 \mathrm{E}-14 \mathrm{~cm} / \mathrm{s}$. The range of measured corrosion rates was $1.8 \mathrm{E}-13-8.9 \mathrm{E}-16 \mathrm{~cm} / \mathrm{s}$. Table C-3 presents the corrosion rates in the various soils for these two metals. Sensitized steels corrode at a rate of 1 to 2 orders of magnitude faster [Gerhold, 1981].

\section{REFERENCES}

[Gerhold, 1981]

Gerhold, W.F., E. Escalante, and B.T. Sanderson, "The Corrosion Behavior of Selected Stainless Steels in Soil Environments," NBSIR 81-2228, National Bureau of Standards, 1981.

[Logan, 1939]

Logan, K.H., "Engineering Significance of National Bureau of Standards Soil-Corrosion Data," Journal of Research of the National Bureau of Standards, Vol. 22, January 1939.

[Romanoff, 1957]

Romanoff, M., "Underground Corrosion," National Bureau of Standards Circular 579, 1957.

[Sullivan, 1988]

Sullivan, T.M., C.R. Kempf, C.J. Suen, and S.F. Mughabghab, "Low-Level Radioactive Waste Source Term Model Development and Testing," NUREG/CR-5204, BNL-NUREG-52160, Brookhaven National Laboratory, 1988. 